\title{
Controlled Wash Water Injection to the Hydrocyclone Underflow
}

\section{Geregelte Waschwassereinspritzung in den Hydrozyklonunterlauf}

\author{
Der Technischen Fakultät der \\ Universität Erlangen - Nürnberg
}

zur Erlangung des Grades

\section{DOKTOR - INGENIEUR}

vorgelegt von

Mohamed Galal Farghaly Aly

Erlangen 2009 
Als Dissertation genehmigt von der

Technischen Fakultät der

Friedrich-Alexander-Universität Erlangen-Nürnberg

Tag der Einreichung: $\quad$ 04.05.2009

Tag der Promotion: $\quad$ 01.07.2009

Dekan: $\quad$ Prof. Dr. Ing. Johannes Huber

Berichterstatter: Prof. Dr. Ing. Thomas Neesse

Prof. Dr. Ing. Abdel-Zaher M. Abouzeid 
To

my parents, wife, daughter, son and to those who love me.

With love and gratitude.

May God bless them. 


\section{Acknowledgements}

The present thesis is based on the work carried out in the Institute of Environmental Process Engineering and Recycling (LUR) of the Friedrich-Alexander-University Erlangen -Nuremberg under the supervision of Prof. Dr.-Ing. Thomas Neesse.

It gives me great pleasure to take this opportunity to acknowledge my indebtedness to all people who have helped me in completing the present work.

First and foremost, I express my profound gratitude to Prof. Th. Neesse for his continuous support, great assistance, constant guidance, enthusiastic encouragement and supervision during my work. Many thanks are also due to him for the good working conditions and active and international academic environment of his institute. I am grateful to him for so much I have learned from him personally. This work would have been impossible without his insight and enthusiasm for the subject.

I am also more than grateful to Dr. J. Dueck for his advice, guidance and his cooperation during all phases of the present research. I owe special thanks for his friendly approach, continued encouragement and valuable discussions. He was exceptionally thorough and a great to deal with.

Thanks are extended to the staff of LUR for their great support and kind assistance throughout the work.

Special thanks are expressed to the committee members: Prof. Dr. Abdel-Zaher M. Abouzeid, Prof. Dr. Rainer. Buchholz, Prof. Dr. Heike Dörnenburg and Dr. Andreas Otto for their valuable criticism and evaluating the present work.

I would like to express my deepest thank and best appreciations to the Egyptian Ministry of Higher Education for the financial support during the research period.

Finally, I would like to express my deepest, warmest and endless gratitude to my parents, wife, and children for their patience, enthusiastically supporting and unlimited encouragement. I learned and understood from each of them what 'A Family' means I am more than lucky to belong to them. 


\section{Table of Contents}

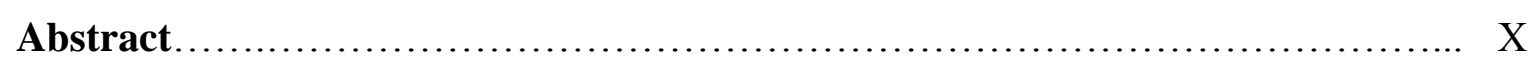

Kurzzusammenfassung ............................................... XI

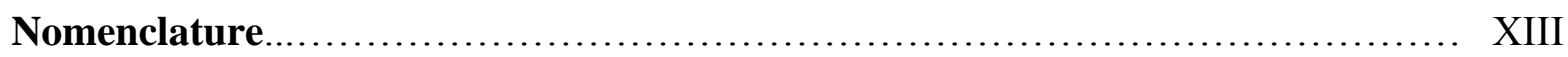

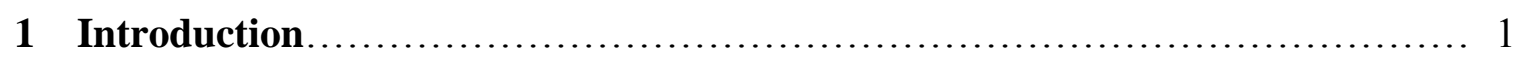

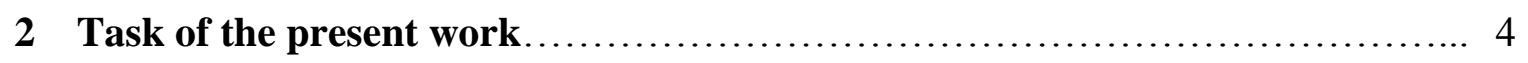

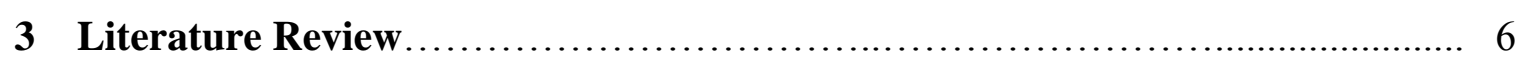

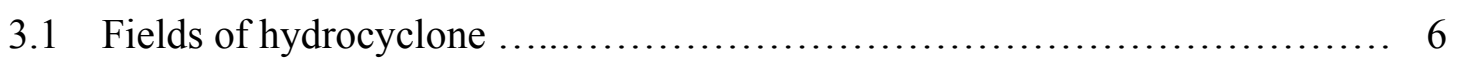

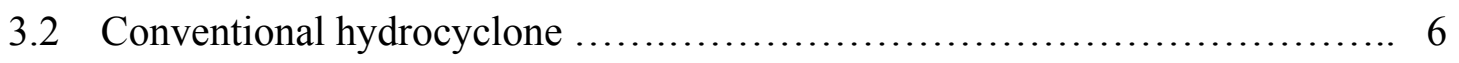

3.3 Principles of hydrocyclone operation............................................. 7

3.4 Fundamentals of the separation in the hydrocyclone $\ldots \ldots \ldots \ldots \ldots \ldots \ldots \ldots . \quad 8$

3.5 Methods for reducing the underflow fines..................................... 10

3.5.1 Hydrocyclone control system to maximize thickening.......... 10

3.5.2 Using multi-stage hydrocyclone separation.................. 14

3.5.3 Cyclone design modification........................... 16

3.5.3.1 Double cyclone.................................. 16

3.5.3.2 The twin vortex (TC) hydrocyclone................ 18

3.5.4 Hydraulic water injection............................... 20

3.6 Conclusion.................................................... 25

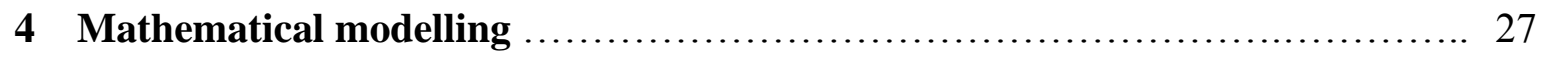

4.1 Formulation of a separation model.................................. 27

4.2 Model of jet injection...................................................... 29

4.3 Definition of the separation curve .............................................. 30

4.4 Model of particle settling in a polydisperse suspension.................... 32 


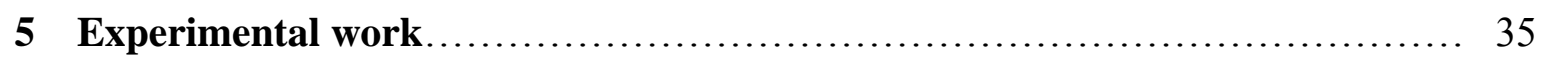

5.1 Material ........................................................... 35

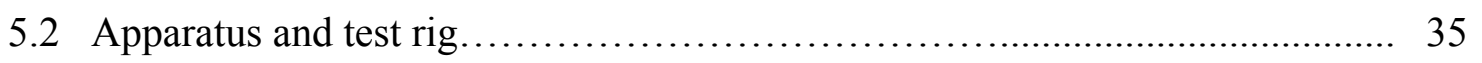

5.2.1 The $50 \mathrm{~mm}$ water injection hydrocyclone....................... 35

5.2.2 Water injection assembly................................. 36

5.2.3 The $50 \mathrm{~mm}$ water injection hydrocyclone test rig.................. 40

5.2.4 Operating the $50 \mathrm{~mm}$ water injection hydrocyclone test rig........... 41

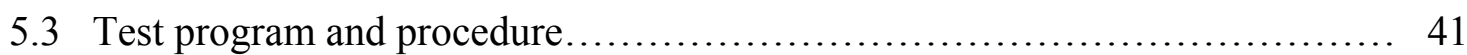

5.3.1 The $50 \mathrm{~mm}$ water injection hydrocyclone test program............... 41

5.3.2 Procedure of water injection experiments ........................ 43

$5.4 \quad$ Particle size analysis............................................... 44

5.4.1 Preparation of the sample for analysis........................... 45

$5.4 .2 \quad$ Sample analysis............................................ 45

6 Data Treatment and error estimation....................................... 46

6.1 Data treatment for separation curve determination...................... 46

6.2 Experimental error estimation.................................... 47

6.2.1 Systematic error sources of the present work.................... 47

6.2.1.1 Sampling procedure................................ 47

6.2.1.2 Volume flow......................................... 48

6.2.1.3 Solids flow......................................... 49

6.2.1.4 Particle size flow..................................... 51

6.3 Statistical error..................................................... 53

6.3.1 Statistical error affects the separation curve determination............ 54

6.3.1.1 Statistical error of mass recovery determination........... 54

6.3.1.2 Statistical error of size analysis...................... 56

6.4 Experimental error evaluation...................................... 56 


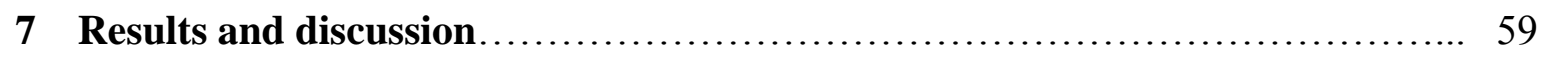

7.1 Investigation of the $50 \mathrm{~mm}$ water only cyclone (without water injection)....... 59

7.2 Investigation of the $50 \mathrm{~mm}$ water only cyclone (with water injection)......... 62

7.2.1 Effect of injection rate on the overflow and underflow flow rates....... 62

7.2.2 Effect of injection rate on the volume split parameter (S) using 3 injection openings..................................... 64

7.2.3 Effect of injection rate on the volume split parameter (S) using 5 injection openings................................... 65

7.3 Classification in the $50 \mathrm{~mm}$ hydrocyclone with water injection............... 67

7.3.1 Effect of injection direction............................... 67

7.3.1.1 Effect of injection direction on the separation efficiency of the fine particles $\left(\mathrm{T}_{0}, \mathrm{~T}_{\min }\right) \ldots \ldots \ldots \ldots \ldots \ldots \ldots \ldots . \ldots 6$

7.3.1.2 Effect of injection direction on the cut size $\left(\mathrm{d}_{50}\right)$ and the imperfection (I) ...................................... 70

7.3.1.3 Effect of injection direction on the solid content and solid recovery.............................................. 71

7.3.2 Effect of injection openings number.............................. 72

7.3.2.1 Effect of injection openings number on the separation efficiency of the fine particles $\left(\mathrm{T}_{0}, \mathrm{~T}_{\min }\right) \ldots \ldots \ldots \ldots .72$

7.3.2.2 Effect of injection openings number on the cut size $\left(\mathrm{d}_{50}\right)$ and imperfection (I).

7.3.2.3 Effect of injection openings number on the solid recovery and solid content......................................... 76

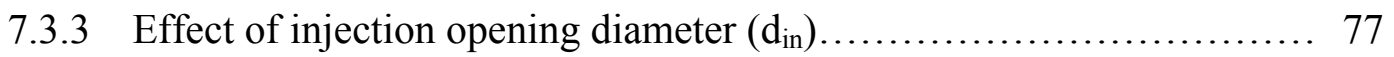

7.3.3.1 Effect of injection opening diameter on the separation efficiency of the fine particles $\left(\mathrm{T}_{0}, \mathrm{~T}_{\min }\right) \ldots \ldots \ldots \ldots \ldots \ldots \ldots \ldots \ldots \ldots$

7.3.3.2 Effect of injection opening diameter on the cut size $\left(\mathrm{d}_{50}\right)$

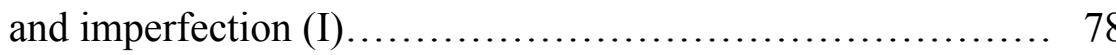


7.3.3.3 Effect of injection opening diameter on the solid recovery and solid content

7.3.4 Effect of injection height from the apex (h)

7.3.4.1 Effect of injection height on the separation efficiency of the fine particles $\left(\mathrm{T}_{0}, \mathrm{~T}_{\min }\right)$.

7.3.4.2 Effect of injection height on the cut size $\left(\mathrm{d}_{50}\right)$ and imperfection (I)

7.3.4.3 Effect of injection height (h) on the solid recovery and solid Content.

7.3.5 Effect of inner vortex finder length (IVFL).

7.3.5.1 Effect of inner vortex finder length on the separation efficiency of the fine particles $\left(\mathrm{T}_{0}, \mathrm{Tmin}\right)$....

7.3.5.2 Effect of inner vortex finder length on the cut size $\left(\mathrm{d}_{50}\right)$ and imperfection (I)

7.3.5.3 Effect of inner vortex finder length on the solid recovery and solid content.

7.3.6 Effect of injection rate (IR) and injection velocity $\left(V_{\text {in }}\right)$. 91

7.3.6.1 Injection at low injection velocities 91

7.3.6.2 Injection at high injection velocities 96

7.3.6.3 Effect of injection rate on the cut size $\left(\mathrm{d}_{50}\right)$ and imperfection (I) 99

7.3.6.4 Effect of injection rate on the solid recovery and solid content 101

7.3.7 Effect of underflow diameter $\left(D_{u}\right)$. 102

7.3.7.1 Effect of underflow diameter on the separation efficiency of the fine particles $\left(\mathrm{T}_{0}, \mathrm{~T}_{\min }\right)$.

7.3.7.2 Effect of underflow diameter on the cut size $\left(\mathrm{d}_{50}\right)$ and imperfection (I)....

7.3.7.3 Effect of underflow diameter on the solid recovery and solid content. 
7.3.8 Effect of overflow diameter $\left(\mathrm{D}_{\mathrm{o}}\right)$

7.3.8.1 Effect of overflow diameter on the separation efficiency of the fine particles $\left(\mathrm{T}_{0}, \mathrm{~T}_{\min }\right)$

7.3.8.2 Effect of overflow diameter on the cut size $\left(\mathrm{d}_{50}\right)$ and imperfection (I)

7.3.8.3 Effect of overflow diameter on the solid recovery and solid content.

7.3.9 Effect of feed pressure (P)

7.3.9.1 Effect of feed pressure on the separation efficiency of the fine particles $\left(\mathrm{T}_{0}, \mathrm{~T}_{\min }\right)$

7.3.9.2 Effect of feed pressure on the cut size $\left(\mathrm{d}_{50}\right)$ and imperfection (I)

7.3.9.3 Effect of feed pressure on the solid recovery and solid content. 114

7.3.10 Effect of feed solid content.

7.3.10.1 Effect of feed solid content on the separation efficiency of the fine particles $\left(\mathrm{T}_{0}, \mathrm{~T}_{\min }\right)$

7.3.10.2 Effect of feed solid content on the cut size $\left(\mathrm{d}_{50}\right)$ and imperfection (I)

7.3.10.3 Effect of feed solid content on the solid recovery and solid 118 content

7.4 Controlled water injection

8 Summary and conclusions

9 Bibliography. 


\section{Abstract}

In hydrocyclones, the classification efficiency is limited by the fines, which are discharged together with the water in the underflow. There have been several attempts focused on improving the washing of the sediment in the conical section of the hydrocyclone. Tangential water injection into the cyclone cone has been applied to displace feed pulp water in the underflow stream and increase the sharpness of the separation. The application of water injection in cyclones has been restricted up to now to special cases and separations in the coarse range. A further disadvantage is its sensitivity to changing feed conditions. The present work presents an improved technique, which was done via an injection at the upper end of the apex or the conical end. An experimental program has been carried out to study the influences of the design and operational parameters which affect the injection process. At the beginning, water only experiments have been done to investigate the effect of the injection on the water distribution through the overflow and underflow openings. After that, the suspension experiments have been made. The results showed that there are optimums conditions of the injection process. Operating at these optimum conditions results in a greater washing effect and reduced consumption of wash water. The process is stabilized by controlled water injection specific to the underflow shape. This controlled wash water injection is applied to kaolin processing for the reduction of kaolin losses in the cyclone underflow. It was found that the water injection through the apex can reduce the fines percentage in the underflow with more than $65 \%$. At the same time, the overflow quality was kept constant and a stable or relatively smaller cut size $\left(\mathrm{d}_{50}\right)$ was achieved.

The experimental data which demonstrate the marked improvement of the separation curves using controlled water injection were presented. These results were supported by a mathematical model describes the injection effect in the hydrocyclone on the basis of the separation model of Schubert and Neesse [1]. The simulation results showed good agreement with the experimental ones. 


\section{Kurzzusammenfassung}

Die Klassierung im Hydrozyklon wird durch den Fehlaustrag der Feinteilchen, die zusammen mit dem Wasser durch den Unterlauf ausgetragen werden, beschränkt.

Es hat mehrere Versuche gegeben, die sich auf die Durchführung einer Sedimentwäsche im konischen Teil des Hydrozyklons konzentrierten. Es wurde eine tangentiale Wassereinspritzung in den Zyklonkonus angewandt mit dem Ziel, das aufgegebene Wasser zum Oberlaufstrom abzuleiten und die Trennschärfe zu erhöhen.

Die Anwendung der Wasserinjektion im Zyklon ist bislang auf spezielle Fälle der Separation im Grobkornbereich beschränkt geblieben. Ein weiterer Nachteil besteht in der Sensibilität dieser Methode gegenüber Fluktuationen im Hydrozykloneingang.

Die vorgelegte Arbeit präsentiert eine weiterentwickelte Technik der Wasserinjektion am oberen Ende der Unterlaufdüse bzw. am Konusende. Das experimentelle Programm wurde darauf abgestimmt, die Einflüsse des Zyklongeometrie und der Prozessparameter, die den Injektionsprozess beeinflussen, zu untersuchen.

Zunächst wurden die Experimente nur mit wassergespeistem Hydrozyklon durchgeführt, um die Wirkung der Injektion auf die Verteilung im Überlauf und Unterlauf zu untersuchen. Dann wurde mit Suspensionen weiter gearbeitet. Die Ergebnisse zeigen, dass es optimale Bedingungen für die Injektion gibt.

Die Arbeit unter optimalen Bedingungen führt zu einer besseren Waschwirkung und reduziert den Waschwasserverbrauch. Der Prozess kann durch eine Regelung der Wassereinspritzung stabilisiert werden.

Diese kontrollierte Waschwassereinspritzung wird in der Kaolinaufbereitung für die Reduzierung der Kaolinverluste im Zyklon-Unterlauf angewendet. Es wurde festgestellt, dass die Wasserinjektion in der Unterlaufdüse den Kaolingehalt im Unterlauf um mehr als $65 \%$ verringern kann. 
Gleichzeitig wird die Überlaufsqualität konstant gehalten und eine stabile oder sogar kleinere Trennkorngröße $\left(\mathrm{d}_{50}\right)$ erreicht. Die experimentellen Daten, die die deutliche Verbesserung der Trennkurven durch kontrollierte Wassereinspritzung zeigen, werden diskutiert.

Diese Ergebnisse über die Wirkung der Injektion im Hydrozyklon wurden durch ein mathematisches Modell auf der Grundlage des Trennmodells von Schubert und Neesse beschrieben [1]. Die Simulationsergebnisse zeigten gute Übereinstimmung mit den experimentellen Daten. 


\section{Nomenclature}

\begin{tabular}{|c|c|c|}
\hline Symbol & Description & Unit \\
\hline$A_{i}$ & Feed inlet area of hydrocyclone & {$\left[\mathrm{mm}^{2}\right]$} \\
\hline$b$ & Centrifugal number & {$[-]$} \\
\hline$c_{j}$ & $\mathrm{j}$-th fraction particle concentration & {$\left[\mathrm{kg} / \mathrm{m}^{3}\right]$} \\
\hline$c_{v}$ & Total volumetric solids concentration in suspension & {$\left[\mathrm{kg} / \mathrm{m}^{3}\right]$} \\
\hline$C_{o}$ & Overflow concentration & {$[\mathrm{kg} / \mathrm{l}]$} \\
\hline$C_{u}$ & Underflow concentration & {$[\mathrm{kg} / \mathrm{l}]$} \\
\hline$d_{25}$ & The cut size with a $25 \%$ fractional recovery in the underflow & {$[\mu \mathrm{m}]$} \\
\hline$d_{50}$ & The cut size with a $50 \%$ fractional recovery in the underflow & {$[\mu \mathrm{m}]$} \\
\hline$d_{75}$ & The cut size with a $75 \%$ fractional recovery in the underflow & {$[\mu \mathrm{m}]$} \\
\hline$d_{\text {in }}$ & Injection opening diameter & {$[\mathrm{mm}]$} \\
\hline$d_{j}$ & $\mathrm{j}$-th fraction particle diameter & {$[\mathrm{m}]$} \\
\hline$d t$ & Separation size & {$[\mu \mathrm{m}]$} \\
\hline$D_{c}$ & Cyclone diameter & {$[\mathrm{mm}]$} \\
\hline$D_{i}$ & Inlet diameter & {$[\mathrm{mm}]$} \\
\hline$D_{o}$ & Overflow diameter & {$[\mathrm{mm}]$} \\
\hline$D_{t}$ & Turbulent diffusion coefficient & {$\left[\mathrm{m}^{2} / \mathrm{sec}\right]$} \\
\hline$D_{u}$ & Underflow diameter & {$[\mathrm{mm}]$} \\
\hline$E_{25 / 75}$ & sharpness index & {$[\%]$} \\
\hline$e(d)$ & Weight fraction of particle size (d) in the feed & [vol. \%] \\
\hline$f_{E}\left(d_{j}\right)$ & Entraining function & {$[-]$} \\
\hline$F$ & Feed solid flow rate & {$[\mathrm{kg} / \mathrm{h}]$} \\
\hline$g\left(c_{v}\right)$ & Empiric correction function & {$[-]$} \\
\hline$h$ & Height of classifier & {$[\mathrm{mm}]$} \\
\hline$h_{\text {in }}$ & Injection height from the upper end of the apex & {$[\mathrm{mm}]$} \\
\hline$h_{o}$ & Height of the overflow & {$[\mathrm{mm}]$} \\
\hline$h_{u}$ & Height of the underflow & {$[\mathrm{mm}]$} \\
\hline$I R$ & Water injection rate & {$[1 / \min ]$} \\
\hline$I$ & Imperfection & {$[\%]$} \\
\hline$k$ & Experimental constant & {$[-]$} \\
\hline$l_{1}$ & Outer vortex finder length & {$[\mathrm{mm}]$} \\
\hline
\end{tabular}




\begin{tabular}{|c|c|c|}
\hline Symbol & Description & Unit \\
\hline$l_{2}$ & Inner vortex finder length & {$[\mathrm{mm}]$} \\
\hline$L$ & Length of the apparatus & {$[\mathrm{mm}]$} \\
\hline$L_{1}$ & Cylindrical length & {$[\mathrm{mm}]$} \\
\hline$L_{2}$ & Conical length & {$[\mathrm{mm}]$} \\
\hline$L_{3}$ & Apex length & {$[\mathrm{mm}]$} \\
\hline$m_{\text {sus }}$ & Suspension mass & {$[\mathrm{kg}]$} \\
\hline$M_{u s}$ & Apex solid capacity & $\mathrm{kg}$ \\
\hline$N$ & Number of injection openings & {$[-]$} \\
\hline$N^{\prime}$ & Degrees of freedom & {$[-]$} \\
\hline$N_{i}$ & Number of independent observation & {$[-]$} \\
\hline$O$ & Overflow solid flow rate & {$[\mathrm{kg} / \mathrm{h}]$} \\
\hline$P$ & Inlet pressure & [bar] \\
\hline$Q_{F}^{0}$ & Feed water flow rate & {$[1 / \mathrm{min}]$} \\
\hline$Q_{O F}^{0}$ & Overflow water flow rate & {$[1 / \mathrm{min}]$} \\
\hline$Q_{U F}^{0}$ & Underflow water flow rate & {$[1 / \min ]$} \\
\hline$r_{H}$ & Hydrocyclone radius & {$[\mathrm{m}]$} \\
\hline$R_{o, j}$ & $\mathrm{j}$-th fraction particle consumption through the overflow & {$\left[\mathrm{kg} / \mathrm{m}^{3}\right]$} \\
\hline$R_{u, j}$ & $\mathrm{j}$-th fraction particle consumption through the underflow & {$\left[\mathrm{kg} / \mathrm{m}^{3}\right]$} \\
\hline$S$ & Split parameter & {$[-]$} \\
\hline$t_{N^{\prime}: \alpha / 2}$ & Student $t$ variable with $N$ degrees of freedom & {$[-]$} \\
\hline$T_{0}$ & $\begin{array}{l}\text { Separation efficiency value of the smallest particle size in the } \\
\text { underflow }\end{array}$ & {$[\%]$} \\
\hline$T(d)$ & Separation efficiency value & {$[\%]$} \\
\hline$T_{\min }$ & Lowest separation efficiency value on the separation curve & {$[\%]$} \\
\hline$u$ & Axial velocity component of suspension & {$[\mathrm{m} / \mathrm{sec}]$} \\
\hline$u(d)$ & Weight fraction of particle size $(d)$ in the underflow & [vol. \%] \\
\hline$u_{s}$ & Sedimentation velocity & {$[\mathrm{m} / \mathrm{sec}]$} \\
\hline$U$ & Underflow mass flow rate & {$[\mathrm{kg} / \mathrm{h}]$} \\
\hline$U i$ & Longitudinal velocity component & {$[\mathrm{m} / \mathrm{sec}]$} \\
\hline$U_{t a n}$ & Mean tangential velocity & {$[\mathrm{m} / \mathrm{sec}]$} \\
\hline$U_{\tan }^{\prime}$ & Fluctuation of tangential velocity & {$[\mathrm{m} / \mathrm{sec}]$} \\
\hline$v$ & Radial velocity component of suspension & {$[\mathrm{m} / \mathrm{sec}]$} \\
\hline
\end{tabular}




\begin{tabular}{|c|c|c|}
\hline Symbol & Description & Unit \\
\hline$V_{\text {in }}$ & Injection velocity & {$[\mathrm{m} / \mathrm{sec}]$} \\
\hline$V_{o}$ & Suspension volume flow rate in overflow & {$[1 / \mathrm{h}]$} \\
\hline$V_{r}$ & Radial component of injection velocity & {$[\mathrm{m} / \mathrm{sec}]$} \\
\hline$V_{r d}$ & Radial velocity in the cyclone & {$[\mathrm{m} / \mathrm{sec}]$} \\
\hline$V_{s, j}$ & Sedimentation velocity & {$[\mathrm{m} / \mathrm{sec}]$} \\
\hline$V_{s t, j}$ & Stokes settling rate & {$[\mathrm{m} / \mathrm{sec}]$} \\
\hline$V_{\text {sus }}$ & Suspension volume & {$[1]$} \\
\hline$V_{\text {tang }}$ & Tangential velocity & {$[\mathrm{m} / \mathrm{sec}]$} \\
\hline$V_{u}$ & Suspension volume flow rate in underflow & {$[1 / \mathrm{h}]$} \\
\hline$w$ & Tangential velocity component of suspension & {$[\mathrm{m} / \mathrm{sec}]$} \\
\hline $\bar{x}$ & Mean value of all measurements & {$[-]$} \\
\hline$x_{i}$ & Measured value & {$[-]$} \\
\hline$z$ & Dimensionless parameter & {$[-]$} \\
\hline$\alpha$ & Underflow discharge angle & [degrees] \\
\hline$\beta$ & Entrainment parameter & {$[-]$} \\
\hline$\delta U$ & Relative error & {$[\%]$} \\
\hline$\varepsilon$ & Turbulent viscosity & {$\left[\mathrm{m}^{2} / \mathrm{sec}\right]$} \\
\hline$\varphi p$ & Solid ratio & {$[-]$} \\
\hline$\eta$ & Dynamic viscosity & {$[\mathrm{kg} / \mathrm{m} . \mathrm{sec}]$} \\
\hline$\lambda$ & Length scale of turbulence & {$[\mathrm{m}]$} \\
\hline$\mu_{l}$ & liquid viscosity & {$[\mathrm{Pa} . \mathrm{sec}]$} \\
\hline$\mu_{m}$ & Effective viscosity of Suspension & {$[\mathrm{kg} / \mathrm{m} \cdot \mathrm{sec}]$} \\
\hline$\pi$ & $3.14159 \ldots$ & {$[-]$} \\
\hline$\rho_{l}$ & Liquid density & {$\left[\mathrm{kg} / \mathrm{m}^{3}\right]$} \\
\hline$\rho_{n}$ & Suspension density & {$\left[\mathrm{kg} / \mathrm{m}^{3}\right]$} \\
\hline$\rho_{s}$ & Solid density & {$\left[\mathrm{kg} / \mathrm{m}^{3}\right]$} \\
\hline$\sigma$ & Standard deviation & {$[\%]$} \\
\hline$\Delta m_{j}$ & $\mathrm{j}$-th fraction particle relative concentration & {$[\mathrm{kg} / \mathrm{l}]$} \\
\hline$\Delta Q_{O F}^{0}$ & Percent injected water goes to the overflow & {$[\%]$} \\
\hline$\Delta Q_{U F}^{0}$ & Percent injected water goes to the uerflow & {$[\%]$} \\
\hline
\end{tabular}




\section{Introduction}

One of the problems of the separation of fines in the hydrocyclone is the presence of the fines in the underflow, such as in the kaolin/feldspar separation in which the kaolin (the fines) represents the final product. In this case more than $25 \%$ of the kaolin is lost in the underflow as tailings and can not be further recovered easily. The separation efficiency of the fine particles in the underflow can be determined from the separation curve, which is considered the most common method of representing the cyclone efficiency.

The separation curve (also called performance curve, partition curve, or Tromp curve) shows the fraction of a material of a specific size $d$ in the feed of the hydrocyclone that reports to the coarse product (the underflow). Figure 1.1 shows the typical representation of the separation curve. In case of fine separation process, the graph is usually plotted on a log-linear scale to emphasise the fine particle sizes.

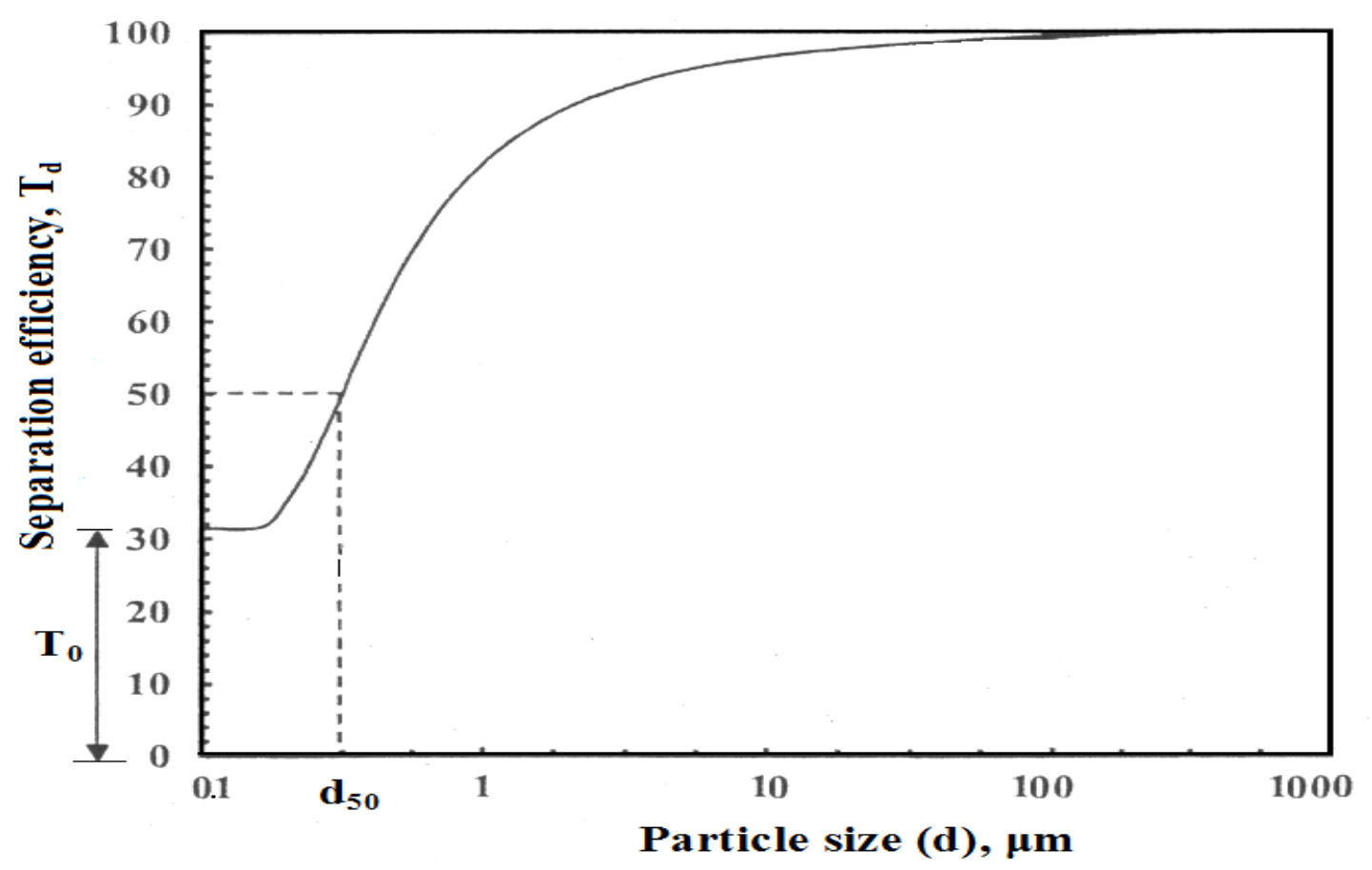

Fig. 1.1: Typical separation curve of the hydrocyclone.

The separation curve is experimentally determined by balancing the particles fluxes for every size class in the products of the apparatus. For any particle size $d$ the separation efficiency value $T(d)$ is calculated from the following formula [2]: 
$T(d)=\frac{U * u(d)}{F^{*} e(d)}$

where, $U$ and $F$ are the mass flow rates in underflow and in feed (in the same units) and $u(d)$ and $e(d)$ are the weight fractions of particle size $d$ in underflow and in feed streams respectively. Eq. (1.1) expresses how to evaluate the performance of a hydrocyclone based on the experimental data. The separation efficiency of the fine particles in the underflow $\left(\mathrm{T}_{0}\right)$ is shown in Fig. 1.1 at particle size $d$ approaching zero. It was found that the percentage of the fines misreported in the underflow $\left(T_{0}\right)$ is related to the feed water recovered in the underflow [3].

Therefore, the smaller the volume of the underflow water, the less is the fines percent associated with it and the better is the sharpness of separation for the fine particles. The sharpness of the separation depends on the slope of the central section of the separation curve; the closer is the slope to the vertical, the higher is the efficiency. The slope of the curve can be expressed by taking the points at which $75 \%$ and $25 \%$ of the feed particles report to the underflow. These are the $d_{75}$ and $d_{25}$ sizes, respectively. The efficiency of separation, or the so-called imperfection $(I)$, is then given by the following expression [4].

$I=\frac{d_{75}-d_{25}}{2 d_{50}}$

where, $\mathrm{d}_{50}$ is the particle size that has an equal probability of reporting to the overflow and the underflow. The imperfection definition of Eq. (1.2) will be used through out the present work to express the separation sharpness. Another definition to calculate the sharpness of the hydrocyclone was developed by Eder [5]. In this definition, the efficiency of the separation is represented by the sharpness of the separation or the so called sharpness index $\left(E_{25 / 75}\right)$ as shown in Eq. (1.3). 
$E_{25 / 75}=\frac{d_{25 c}}{d_{75 c}}$

\section{$(1.3)$}

Accordingly, different methods were tried to improve the sharpness of the separation concerning the fines by reducing the amount of feed water goes to the underflow. These methods were mainly as follows: automatic control to maximise thickening [6], using multi-stage hydrocyclone separation [7], cyclone modifications [8], and water injection through the conical part [9]. Most of these methods still have some restrictions in the laboratory and industrial scales and the others have some disadvantages especially with the accompanying increase in the cut size.

Therefore, the aim of the present work was to improve the fine separation process in the hydrocyclone to reduce the fines percentage in the underflow without changing the cut size $\left(d_{50}\right)$. This was made by using the water injection technique near the apex of the hydrocyclone. The motivation was to reach the optimum position and design of the injection part which achieves high washing effect, and improves the recovery of fines without changing the overflow quality (the cut size $\mathrm{d}_{50}$ ). 


\section{Task of the Present Work}

The present work was carried out at the Environmental Process Engineering and Recycling Institute (LUR), Freidrich-Alexander University, Erlangen, Germany where a scientific school of hydrocyclone with the following preconditions is found:

- Practical experience in the field of hydrocyclone separation

- Development of theoretical models which simulate the separation process in the hydrocyclone

- Technical base (different types and sizes of cyclones and test rigs)

- Modern analysis instruments

Based on the above mentioned preconditions, this work presents a new version of water injection cyclone without any additional mechanical parts or truncated cone to be inserted through the cyclone. The injection was made just above the upper end of the hydrocyclone apex. The design of the injection part was commercially carried out at the design and manufacturing branch in the institute.

The aim of the present work was the optimization of the water injection cyclone on the base of a modified separation model developed by Schubert and Neesse [1]. The effect of the injection on the water distribution through the overflow and underflow openings of the hydrocyclone was studied using only water as feed. Then the suspension experiments were carried out. The injection was varied concerning the injection location, injection direction, injection rate, and number of injection points. The optimum injection conditions are obtained, then the influence of the cyclone parameters on the injection performance was investigated. The investigated parameters were as follows:

- Injection direction

- Number of injection openings

- Diameter of injection openings

- Injection height from the apex

- Extension of vortex finder length inner the cyclone

- Injection rate

- Underflow diameter

- Overflow diameter

- Feed pressure 
- Feed solid content

Parallel to this extensive experimental program, a mathematical model using Fluent 6.2 software to describe the effect of water injection on the separation process within the hydrocyclone was developed [10]. This model was based on the separation model of Schubert and Neesse [1]. The experimental results and the calculated results were compared to show the degree of fitness between both results. 


\section{Literature Review}

\subsection{Fields of Hydrocyclone}

Hydrocyclone is considered an important industrial separator. It has been used for more than 150 years due to its simple design, small volume, low cost, easy operation, and low maintenance requirements. Besides a large amount of applications in mineral and mining processing [11], hydrocyclone has been used recently in an increasing number of applications in an environmental engineering [12-15], petrochemical engineering [16-21], food engineering [22-25], electrochemical engineering [26,27], bioengineering [28-30], and pulping process [31,32]. Unlike centrifuges, which use the same centrifugal forces, cyclones have no moving parts and the necessary vortex motion is performed by pumping the fluid under pressure into the cyclone body.

\subsection{Conventional Hydrocyclone}

The principle design, cone- cylinder cyclone with a single tangential inlet and two product outlets (the overflow product and the underflow product) is expressed as the conventional cyclone as shown in Fig. 3.1. It is used in most mineral processing plants for various applications which include classification, thickening, desliming, dewatering and density sorting. The designs of the various cyclones differ depending on the application for which the cyclone is designed, but the most common design consists of a cylindrical part joined to a conical part with variations in the cylindrical length $\left(0.66 \mathrm{D}_{\mathrm{c}}-2 \mathrm{D}_{\mathrm{c}}\right)$, cylindrical diameter $(10 \mathrm{~mm}-150 \mathrm{~mm})$, and the cone angle $\left(9^{\circ}-25^{\circ}\right)[8]$. 


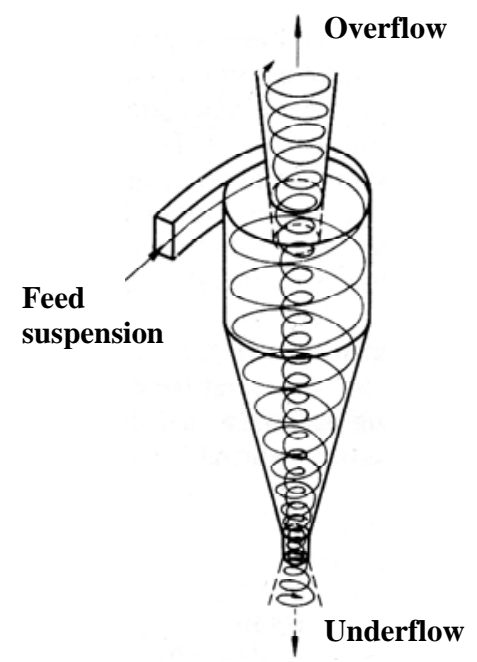

Fig. 3.1: Conventional hydrocyclone.

\subsection{Principles of Hydrocyclone Operation}

The basic separation principle employed in hydrocyclones is the centrifugal sedimentation. Feed slurry is introduced under pressure via the tangential inlet and is constrained by the geometry of the unit to move into a circular path. This creates the opposing outward centrifugal and inwardly acting drag forces which result in a spiral flow pattern. An air core develops along the vertical axis which is connected to the atmosphere through the spigot, but the part created by dissolved air is coming out of solution in the low-pressure zone [33,34] [35]. According to the (Simplified) imaginations, for the fine and light particles, the inward drag forces tend to dominate. Hence these particles move towards the vertical axis, join the innermost spiral and are swept up by the central current into the overflow opening. On the other hand, the large and heavy particles will experience a greater centrifugal force. These particles tend to move to the cyclone periphery, join the outermost spiral and move downward toward the spigot (underflow opening). Modern separation models consider the process-determining role of turbulence. The major effect of turbulence is that during classification in a hydrocyclone, a considerable amount of fines reports into the underflow coarse due to the hydraulic entertainment and the short circuiting [36]. These particles have been directly correlated to the amount of feed pulp water recovered to the underflow stream [2]. 
Various aspects of the hydrocyclone (influence of design and operating parameters, mathematical modelling and simulation, and design modification) have been thoroughly studied by many researchers [37-42] in an effort to improve or more efficiently control its performance which is expressed by illustrating the separation efficiency curve of the separation process of the hydrocyclone.

\subsection{Fundamentals of the Separation in the Hydrocyclone}

Many researchers have attempted to calculate particle trajectories in the hydrocyclone and to derive formulae for calculating the separation size and the efficiency of separation. There are some general theories which have been developed to describe the size classification performance of a hydrocyclone. The first theory (equilibrium orbit theory) was developed by Kelsall [43]. Rietema [44] who have some objections and doubts about Kelsall's theory developed another theory for the cut size $\mathrm{d}_{50}$ known as the residence time theory. These theories exist for the prediction of the performance of hydrocyclone operated at low feed solids concentrations (less than 1\% by volume) and low ratio of underflow flow rate/ feed flow rate. These studies were based upon some simplifying assumptions, one of which being that no hindered settling occurs in hydrocyclone. Fahlstrom [45] investigated the classification of solids with higher concentration (up to $20 \%$ by volume). The results were explained in terms of the so-called crowding theory.

The effect of turbulence on the separation in Hydrocyclone has been of concern to researchers ever since the early work of Driessen [46]. One aspect of interest is how turbulence modifies the tangential velocity profiles. Rietema [47] made a detailed investigation into this problem and estimated the turbulent viscosity with the aid of tangential velocity profiles which is measured by Kelsal. This was done using a dimensionless parameter $z$ :

$z=V_{r d} \frac{V}{\varepsilon} r_{H}$

where, $V_{r d}$ is the radial velocity in the cyclone, $r_{H}$ is the radius of the cyclone and $\varepsilon$ is the turbulent viscosity. 
Schubert and Neesse and co-workers [48-51] were the first to propose a separation model based on two-phase turbulent flow. They assumed a homogeneous, stationery turbulence field, with particle moving under Stock's law. They also assumed that the particles are smaller than the smallest eddies and that their concentration is sufficiently low. According to Schubert and Neesse and other researchers [52-58], the particle transportation consists of sedimentation flux superimposed on the turbulent diffusion flux. For mathematical convenience, they also assumed the centrifugalforce field to be homogeneous. Schubert and Neesse derived two general models for turbulent cross-flow classification depending on the way the underflow and overflow streams are removed. These models are the suspension partition model and the suspension tapping model. They applied the tapping model to the separation in a cyclone, with the resulting equation for the cut size. This correlation considers the first which takes into account the effect of the feed solids on the separation in a hydrocyclone.

This contribution from Schubert and Neesse is concerned with the case of higher solids concentrations where the underflow orifice may restrict the free discharge of the solids. They propose the existence of a limit in the solids loading and propose an empirical correlation for the discharge capacity of the underflow orifice. This brings their work into the realms of the crowding theory considered before.

Neesse and Schubert [1], and Neesse et al. [59] calculated the separation curve using the separation model of tube cross flow separation. They introduced a hydrocyclone model based on the equilibrium of sedimentation and turbulent transport in the hydrocyclone. The result of this model is the following equation for the separation curve of the hydrocyclone.

$$
T(d)=\frac{1}{1+S \exp \left[-\frac{r_{H}}{D_{t}} u_{S}(d)\right]}
$$

where, $T(d)$ is the separation efficiency value (the probability that a particle of size $\mathrm{d}$ is discharged in the underflow), $S=\mathrm{V}_{\mathrm{o}} / \mathrm{V}_{\mathrm{u}}$ is the volume split (overflow volumetric 
flow to underflow volumetric flow), $r_{\mathrm{H}}$ is the hydrocyclone radius, $u_{\mathrm{s}}$ is the sedimentation velocity and $D_{\mathrm{t}}$ is the coefficient of turbulent diffusion.

The cut size $\mathrm{d}_{50}$ of the cyclone is usually defined as that particle size on the separation curve for which $50 \%$ of particles of the feed report to the underflow, i.e., particles of this size have an equal chance of going either with the overflow or underflow. This point is usually referred to as the $\mathrm{d}_{50}$ size [60].

According to Schubert and Neesse [1], the cut size can be calculated using the following equation:

$d_{50}=k \sqrt{\frac{\eta D \ln \left[0.91(D o / D u)^{3}\right]}{(1-\varphi p)^{3}(\rho p-\rho f) \sqrt{P / \rho_{n}}}}$

where,

$d_{50}$ is the cut size, $k$ is the experimental constant, $\eta$ is the dynamic viscosity, $D_{o}$ is the overflow diameter, $D_{u}$ is the underflow diameter, $\varphi_{p}$ is the solid ratio, $\rho_{p}$ is the solid density, $\rho_{f}$ is the liquid density, $p$ is the inlet pressure, and $\rho_{n}$ is the suspension density.

\subsection{Methods for Reducing the Underflow Fines}

Many researchers [5-8] have attempted many trials to improve the hydrocyclone separation efficiency by reducing the amount of pulp water recovered in the underflow stream and accordingly decreasing the fines portion misreported to the underflow product which expressed as the separation efficiency value $\left(T_{0}\right)$ on the separation curve. The main procedures for achieving this purpose were the increase of the solids content (higher thickening) in the underflow and the water injection in the conical part.

\subsubsection{Hydrocyclone Control System to Maximize Thickening}


One of the methods which were used in reducing the amount of the underflow water is the use of the thickening process. The required duty in this case is the maximum removal of suspended solid from suspending liquid. This process aims to operate the hydrocyclone at high solid content and the cyclone is therefore designed to give a maximum efficiency for all sizes of particle that will be fed to it [8]. This method may often be utilized in combining the cyclone with another separator to give technically and economically superior results.

The discharge shape of the underflow has been the subject of the investigations by Neesse et al. [59] and Plitt et al. [61]. In this connection, the dependency of the discharge shape on the air core formation has been examined by Concha et al. [62], Dyakowski and Williams [63] and Barrientos et al. [64]. Further, the influence of rheological effects on the performance of hydrocyclones at higher solid content has been investigated by Yopps et al. [65] and Dyakowski et al. [66].

According to Dueck at al. [67-70], the discharge shape of the underflow can be described with the discharge angle $\alpha$ as presented in Fig. 3.2. This angle $\alpha$ is to be computed according to Eq. (3.4) as follows:

$\alpha=\arctan \frac{v}{u} \approx \arctan \left[\frac{\rho_{m} \frac{D_{u}}{2} w^{2}}{\mu_{m} u}\right]$,

where $u, v$, and $w$ are the velocity components of the suspension in axial, radial and tangential direction, $\rho_{\mathrm{m}}$ the density, $\mu_{\mathrm{m}}$ the effective viscosity of the suspension and $D_{u}$ the apex diameter. These quantities were computed at the apex exit using LUCY simulation program with the data of a $150 \mathrm{~mm}$ hydrocyclone. According to Eq. (3.4), the underflow discharge can be regulated by varying the underflow diameter $D_{\mathrm{u}}$ using a control apex as demonstrated by Bradley [8] and Trawinski [71]. 


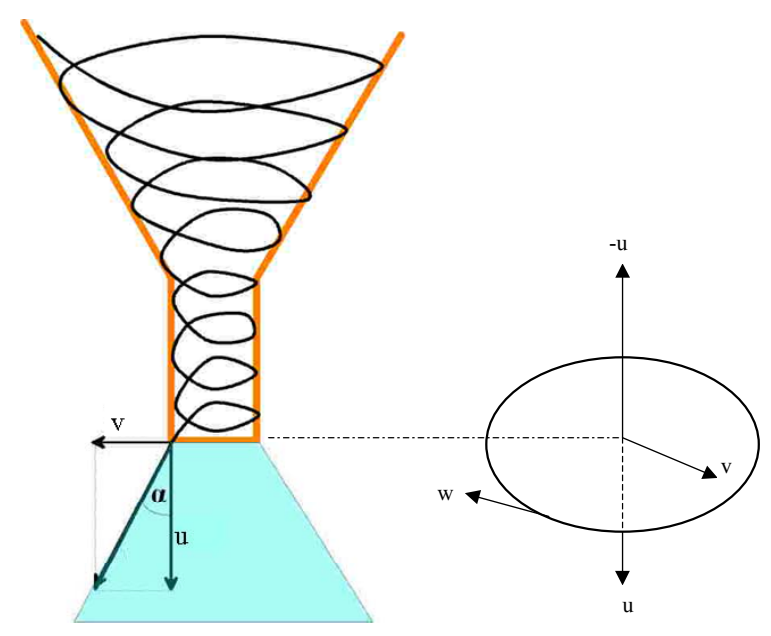

Fig. 3.2: Scheme of the discharge angle of the hydrocyclone underflow, after Dueck et al [67].

Trying to put these concepts into effect, a new control system was developed by Neesse and Donhauser [72], Neesse et al. [73] and Schneider et al. [74] at the Laboratory of Environmental Process Engineering and Recycling Institute, FriedrichAlexander University, Erlangen, Germany. The principle of this control system is shown in Fig. 3.3. 


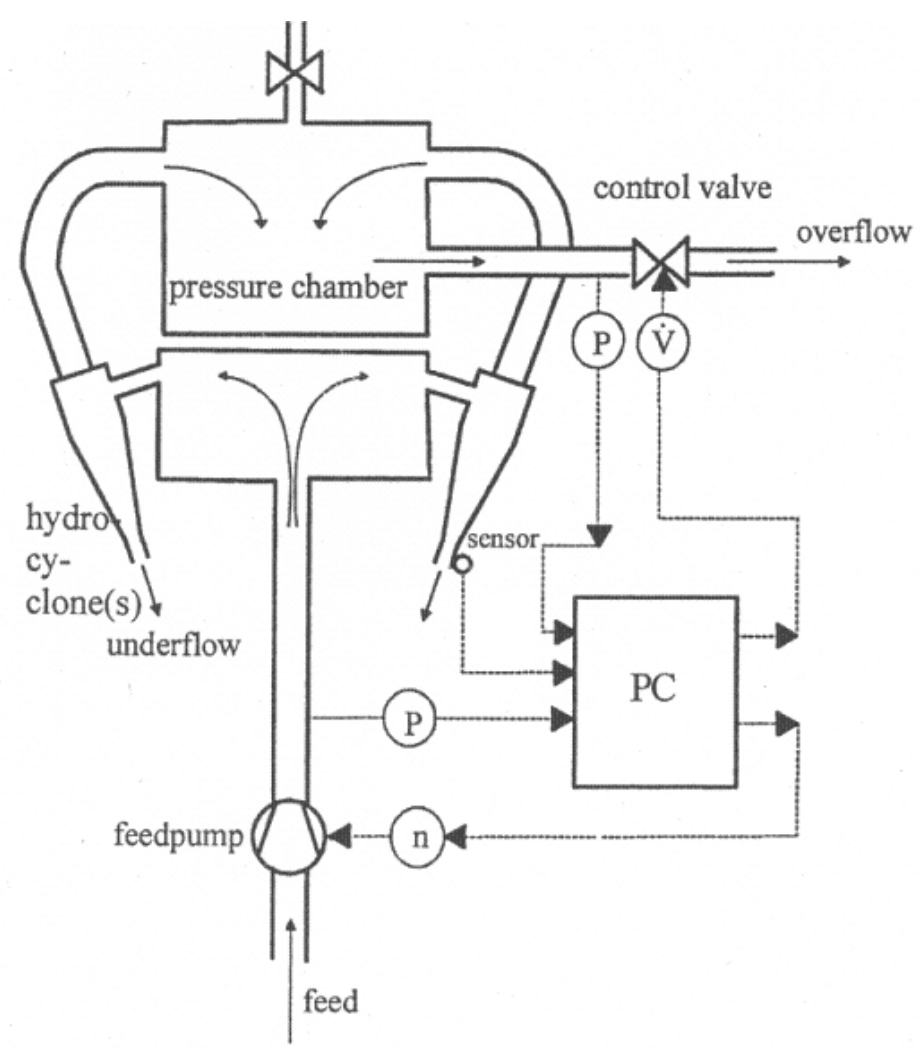

Fig. 3.3: Principle of a computer controlled hydrocyclone battery, after Neesse and Donhauser [72].

Using this system, the operating state of the hydrocyclone can be stabilized or optimized by final control elements regulating the volume split and the feed pump speed as shown in Fig. 3.4. In dilute flow separation Fig. 3.4a. with its typical spray discharge, a continuous air core can be observed that extends to the underflow. The separation presents high solids recovery in the underflow as advantage but low solid concentration resulting in more fines being swept in the underflow.

In dense flow separation Fig. 3.4b, more solids are stored in the conical part of the hydrocyclone. They are partially moved towards the overflow, consequently reducing solids recovery to the underflow. The air core does not extend over the complete hydrocyclone length and oscillates intensively.

The transition state Fig. 3.4c, between spray and rope discharge presents both the advantages of high solids recovery and high solids content in the underflow. Therefore, the new control concept is focused on maintaining this state. 


\begin{tabular}{|c|c|c|c|}
\hline Case & $\begin{array}{c}\text { a.) Dilute flow } \\
\text { separation }\end{array}$ & $\begin{array}{c}\text { b.) Dense flow } \\
\text { separation }\end{array}$ & $\begin{array}{c}\text { c) Transition } \\
\text { state }\end{array}$ \\
\hline $\begin{array}{c}\text { Sischarge form } \\
\text { underflow } \\
\text { discharge }\end{array}$ & Spray & Rope & Transition \\
spray/rope
\end{tabular}

Fig. 3.4: Hydrocyclone operating states, after Schneider et al. [74]. 
Most of the hydrocyclone control methods were based on the adjustment of the volume of the underflow water by reducing the underflow apex diameter. All these methods require the installation of a control valve at the underflow to create back pressure. At high underflow back pressure the rope flow which replaces the air core may cause re-entrainment of solids in the apex. Moreover, restricting the underflow apex is critical because it makes the underflow discharge closer, and hence causes blocking. Such systems are also subject to severe wear and require extra maintenance.

Concerning the volume split control which has been used in reducing the amount of fines in the underflow, the results were not satisfying concerning the minimum fines percent achieved in the underflow. Although the control system can be used to maximise the thickening with stabilising the cut size, it must be used with another technique to reduce the fines markedly in the underflow. This system is mainly applied to obtain the same overflow quality (no coarse particles to be separated in the overflow) under different feed conditions at a stable cut size. The design and arrangements of the control system is an extra discussion topic from the economical point of view if this system is installed and used only for reducing the fines in the underflow.

\subsubsection{Using Multi-stage Hydrocyclone Separation}

The multi-stage hydrocyclone was used to avoid the disadvantages of restricting the underflow apex. It was possible to reduce the underflow fines by using a series of hydrocyclones. The subsequent classification of the underflow in the next stage resulted in low fines in the final underflow.

Peterson and Herbst [75] tested possible configurations for two-stage hydrocyclone classification in grinding mill circuits to minimise the over grinding of the fines entrained in the underflow discharge. In the first case, make-up water was added to the underflow of the first hydrocyclone and fed to the second hydrocyclone. The two overflows were combined to give the final product. The underflow from the second cyclone was returned to the mill as shown in Fig. 3.6a. In the second case, the overflow stream of the first hydrocyclone plus any additional make-up water was the feed to the second hydrocyclone of the circuit. The two underflows were combined 
and returned to the mill while the overflow from the second hydrocyclone was sent to the flotation circuit as can be shown in Fig.3.6b.

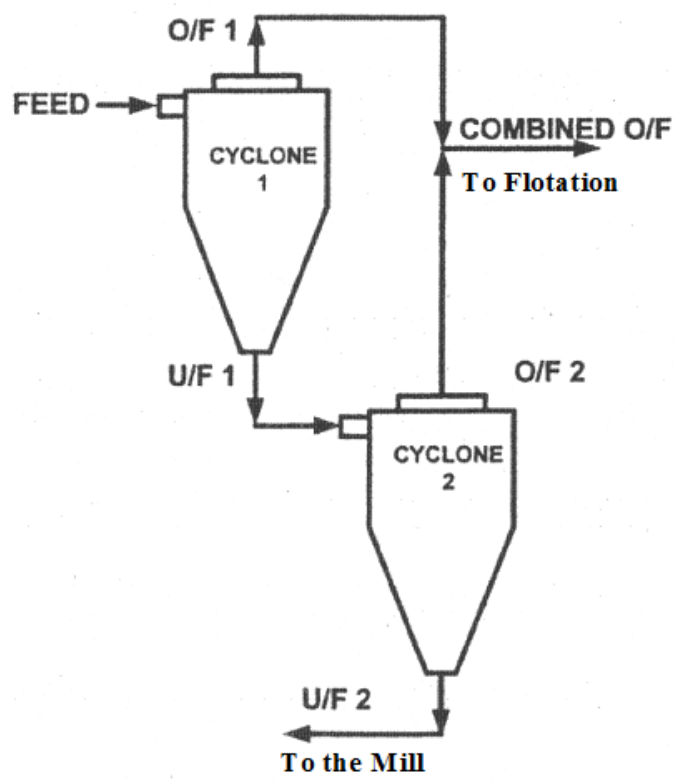

(a)

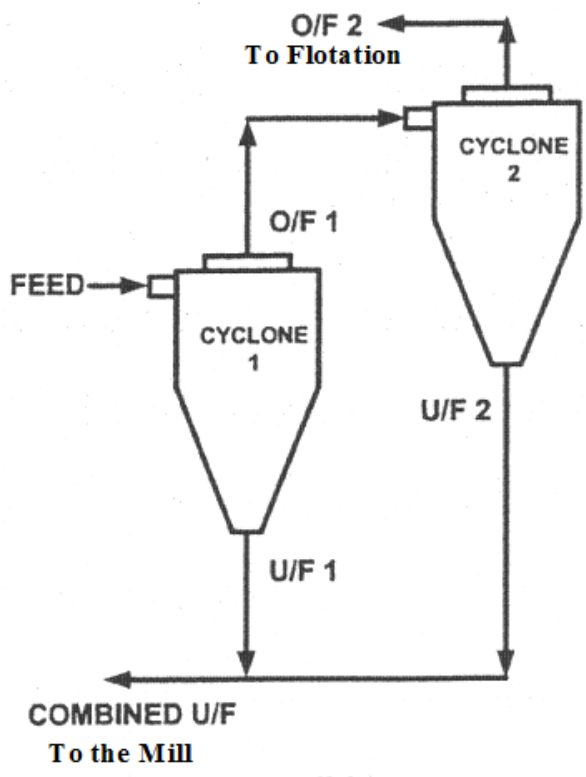

(b)

Fig. 3.6: Two-stage hydrocyclone classification configurations.

It was found that, the first case improved the separation since fine particles that bypass the first hydrocyclone are fed through a second hydrocyclone hence lowering the overall by-pass of fine particles through the hydrocyclone. The second case was thought to give poorer separation since fine particles were given two opportunities to short-circuit into the underflows of the two hydrocyclones.

A case study using a two- stage cycloning carried out at Rakha Copper Concentrator, India was reported by Rao et al. [76]. According to this study, the classification systems in series improved the overall classification performance, reduced the circulating load and further resulted in considerable energy saving. However, this arrangement needed extra sump and pumping systems with additional maintenance. The two-stage classification circuit was reported to be sensitive even for small changes in plant operation. 
In accepting the by-pass problem, Firth et al. [77] proposed the use of a three-stage counter-current cyclone circuit whereby the underflow streams of the primary and secondary cyclones were retreated and the secondary and tertiary overflow streams recycled to the previous feed sumps. Efficiency was found to improve significantly while the amount of ultrafine particles in the underflow stream was decreased. However, the circuit requires additional pumping and an increased number of cyclone banks that create particle attrition and negative effects on the overall process economies.

It should be noted that the performance of the two-stage classification is not compared with the performance of the single-stage hydrocyclone. This was not available in the reviewed work especially in conjunction with a closed grinding circuit followed by a flotation circuit. This information was difficult to achieve on an industrial plant as well as at a pilot plant due to the cost and the time required to change the circuit configuration.

The two or multi-stage cyclone also suffers from the difficulty of having many units to control. Most of the presented two-stage configuration systems were used to either reduce the fine material into the underflow or reduce the coarse material into the overflow. No configuration had a capability of simultaneously achieving both purposes. Another disadvantage of the multi-stage cyclone is the addition of water to underflow which might not be good for applications where the control of solid concentration is critical.

\subsubsection{Cyclone Design Modifications}

In an attempt to avoid the above mentioned disadvantages of multi-stage hydrocyclones, many researchers [78-80] have tried to make some modification of the hydrocyclone to fulfil the requirements for special applications. Most of these modifications were mainly to reduce the amount of fines reported to the underflow discharge. 


\subsubsection{Double Cyclone}

As can be shown in Fig. 3.7, the double-cyclone (cylinder-cyclone) system consists of a cylinder, functioning as a deslimer or predensifier, tangentially discharging the underflow product directly to a cyclone which is connected to the cylinder. As there is no conical part the underflow density is not as high as with the standard cyclone. These cylinder-cyclone units have been used for the pre-treatment of the flotation feeds, desliming, and classifying for gravity concentration circuit feeds.

A simple cylinder-cyclone has been available for many years From Krebs Engineers Company and various uses have been propounded [8] [81]. For fine coal cleaning, Restarick [79] has developed a cylinder-cyclone system incorporating a variable concentric aperture valve between the cylinder and the cyclone units Fig. 3.7. By adjusting this interstage flow control valve, the cut size of the cyclone can be varied as required. This system has successfully classified coal and mineral slurries prior to desliming tests in small-scale continuous flotation circuits. This system still has limited application in large scale operations.

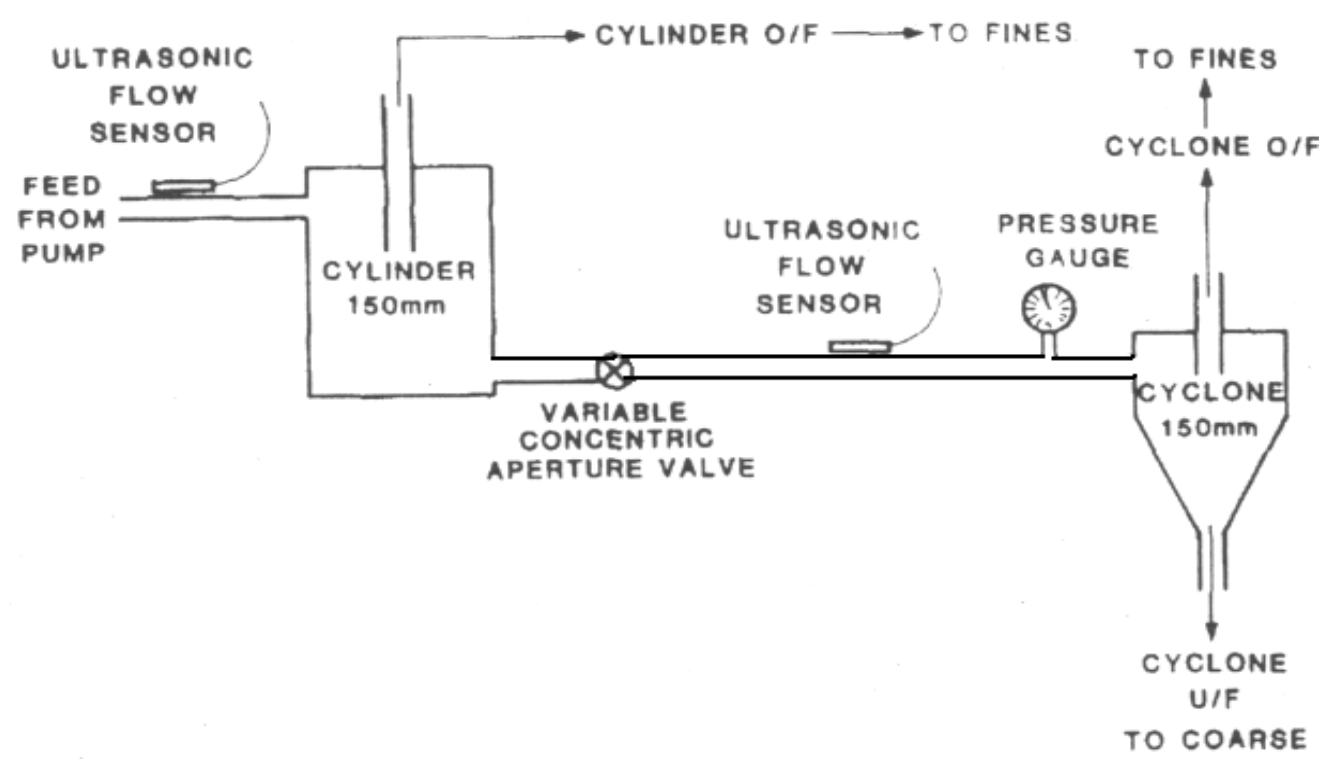

Fig.3.7: Double-cyclone (cylinder cyclone), after Restarick [79].

The coal separation curves for the cylinder, the cyclone, and the combined unit (i.e. when both cylinder and cyclone overflows are combined as the fine product) are 
shown in Fig. 3.8. The cut size $\left(d_{50}\right)$ for the combined unit is primarily determined by the cyclone separation size and this can be shifted to the coarser or finer range.

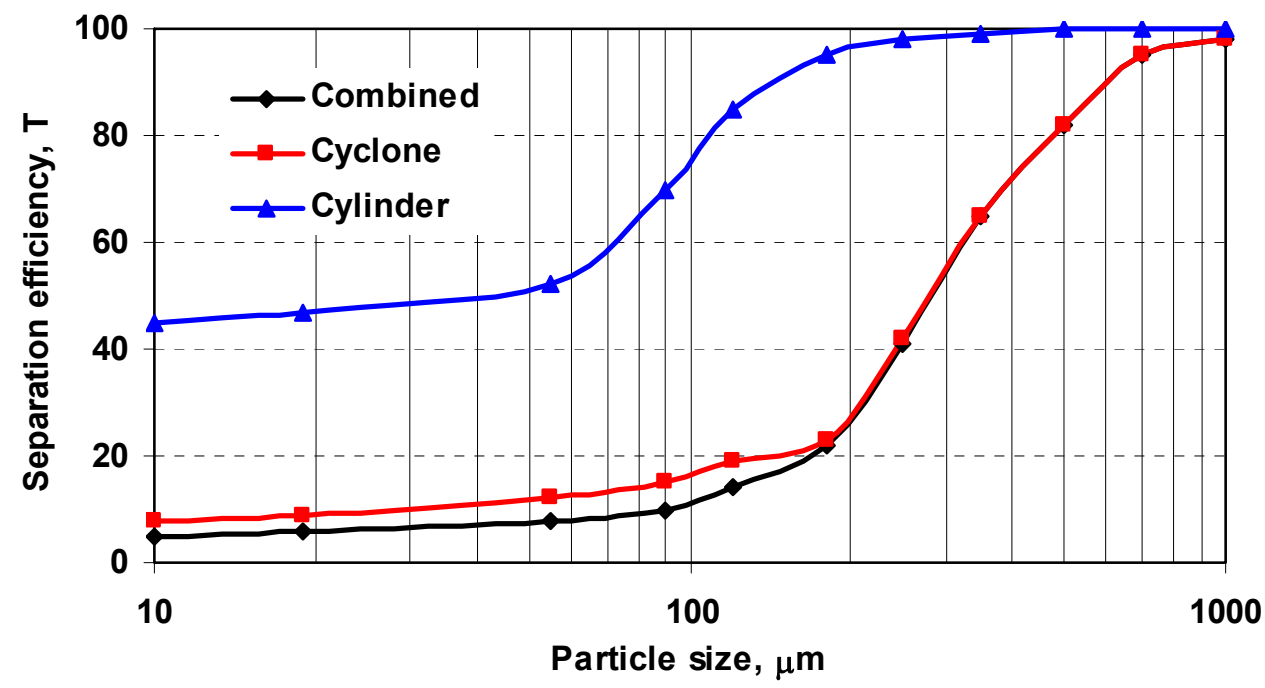

Fig. 3.8: Separation curves of the Double-Cyclone, after Restarick [79].

In such system, as there is no conical part, the underflow density is not as high as with the normal cyclone. Closing the valve will increase the pulp density in the second stage (cyclone). This may decrease the feed flow rate and increase the cut size. This control valve is sensitive and can not be adjusted exactly to obtain the same conditions at every time to be operated.

\subsubsection{The Twin-Vortex (TC) Hydrocyclone}

The twin-vortex cyclone is a combination of two different cyclones. The main aim of this type of cyclone is to reduce the high by-pass fraction and hence reduce the amount of fines which report to the underflow. Heiskanen et al. [82] designed the TC cyclone based on the idea of water replacement which first was proposed by Kelsall [78]. The first part of the TC cyclone is a conventional cyclone in which the conical section is replaced with a cylindrical part which has a larger diameter than the main body as can be seen from Fig. 3.9. This cylindrical part, which is the washing section, has an adjustable central cone with water addition holes. The second part of the TC is an ordinary hydrocyclone for solid-liquid separation to produce a cleaner underflow and a dilute fine overflow. This overflow can be circulated to dilute the primary feed 
which results in an improved classification. During the separation in the first part of the TC cyclone, the coarse product enters the washing section from the gab between the cone and the cyclone body. Water is added to the expanded body in such a way that a zone is created where the pulp density is lowered just at the gap. Due to the difference in the pulp density and depending on the volume balance in the washing unit, water will move upwards. This creates a balance between the forces resulting from the downward vertical velocity and upward water velocity. As a result, the small and light particles in the downward stream are swept back upward into the main body volume. Heavy and light particles move down the zone. They are then removed to the second part of the TC cyclone that separates material into the final coarse product and water that may contain some fines. Figure 3.10 shows the separation curves of the twin-vortex cyclone compared with the conventional one.

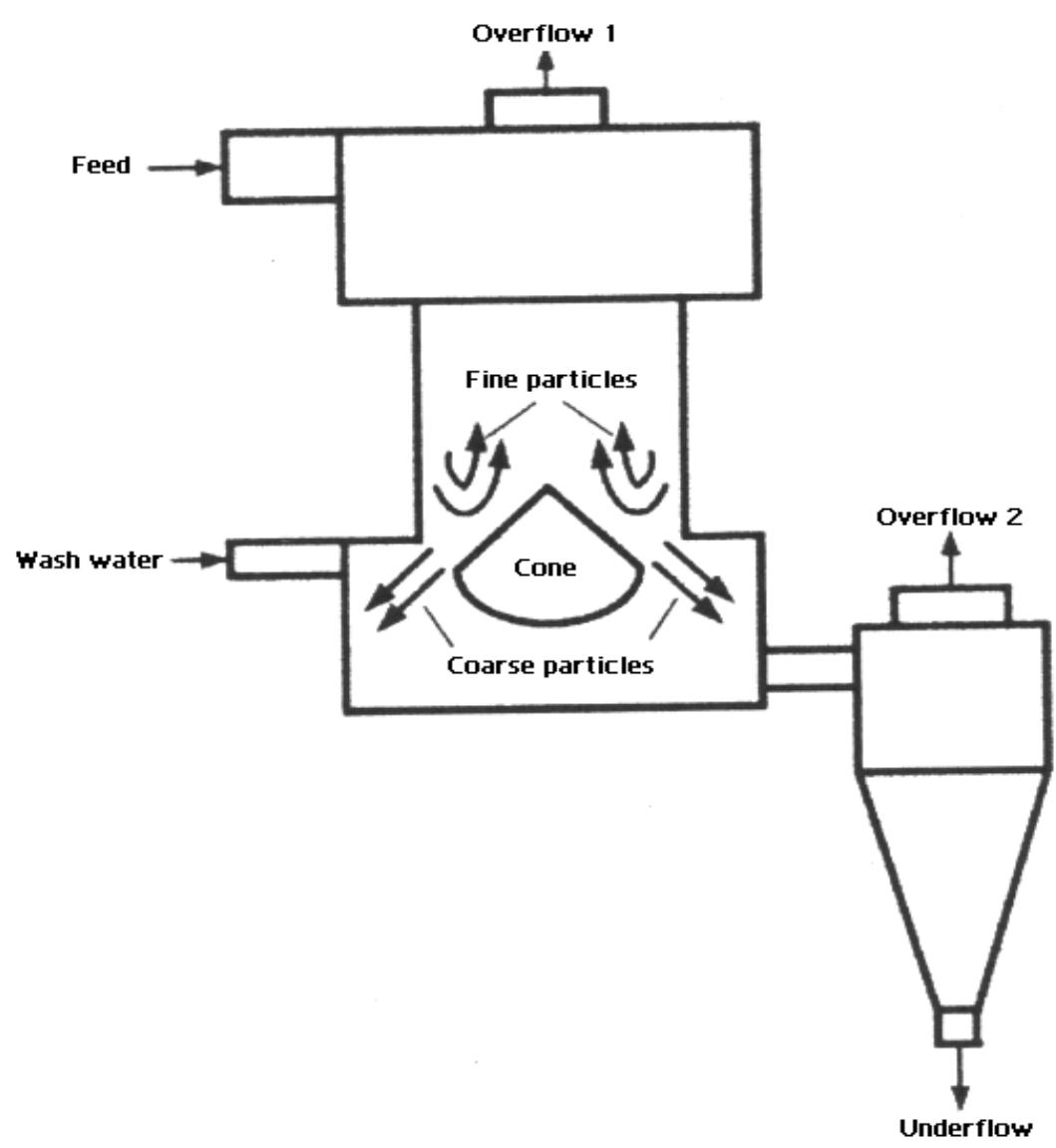

Fig. 3.9: Twin-vortex (TC) cyclone. 


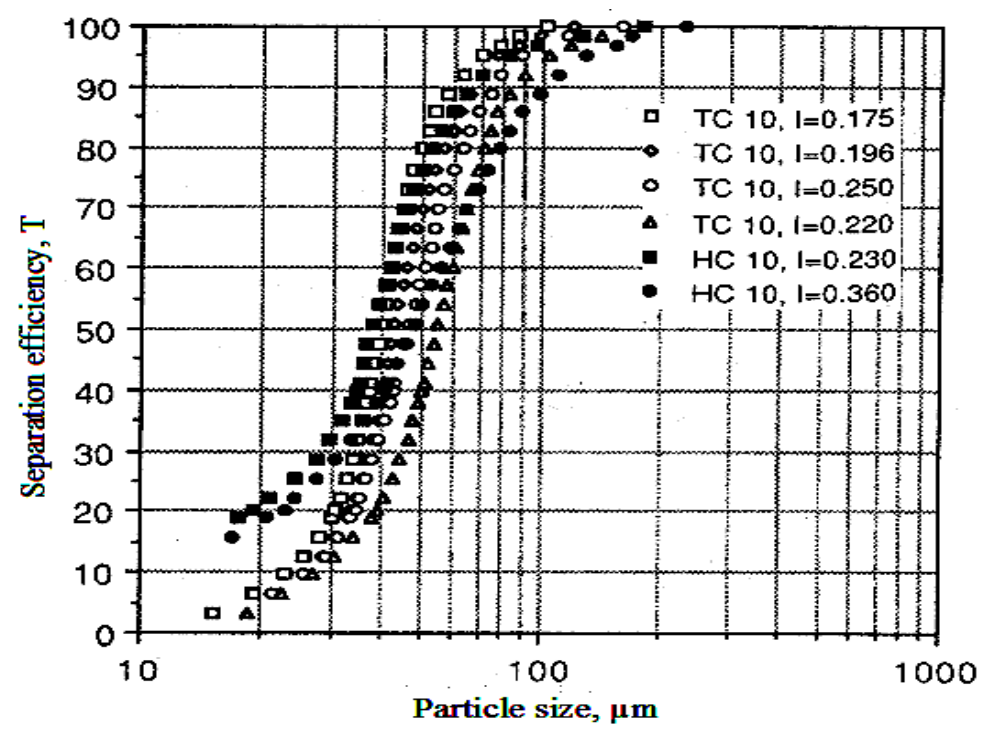

Fig. 3.10: Twin-vortex cyclone separation curves.

The most outstanding improvement in the use of the TC cyclone is the decrease of the by-pass of fines to the underflow. Despite this improvement, the two overflows and the underflow may be too dilute for subsequent processes, rendering it impractical for industrial applications. Strictly speaking, the TC cyclone is a modified two stage cyclone system which fulfils the objective of obtaining an underflow with low fines but might still suffer from coarse material in the overflow stream. In the case of the twin vortex cyclone, it was necessary to add large amounts of water to achieve the objective of the work. Due to this large amount of water, the underflow pulp density is decreased. Thus, it was not possible to recirculate it (the coarse product) again to the mill to further liberate the valuable minerals. This is due to the high dilution which may lead to a drop off in milling capacity. It was also reported that the cut size for TC cyclone increased due to the dilution. It can be mentioned that, the TC cyclone is a modified two stage cycloning system which fulfils the objective of obtaining an underflow devoid of by-pass fines but might still suffer from coarse material in the overflow stream.

Generally, it can be reported that the cyclone design modification is still up to now used for special cases or treatment for special purposes and can not be applied in the wide range of hydrocyclone applications. Most of these modified cyclones were used 
in the coal treatment, milling circuits and in the flotation circuits. More over, these modified cyclones need more constructional area for the test rigs, special maintenance for the spare parts of these units, and special arrangements for the hydrocyclone feed and products. This requires more capital and operating costs. Due to these disadvantages in the TC operation, hydrocyclone lose its main advantage which presents it as a valuable apparatus in the field of separation.

\subsubsection{Hydraulic Water Injection}

Another method for reducing the amount of fines that reports to the underflow is to decrease the by-pass fraction, which usually ranges between $20 \%$ and $40 \%$ of the feed. Several attempts have focused on the washing of the slurry in the conical section of the hydrocyclone with clear water in either single or multistage cycloning [82]. In most of these cases, the by-pass fraction was reduced. However, the cut size $\left(d_{50}\right)$ increased due to disturbances of the flow pattern caused by the water addition.

The water injection into the conical part of the cyclone has been applied to displace feed pulp water in the underflow stream and increase the sharpness of the separation [83]. The process was first used in the paper industry [8]. In this case avoidance of loss of good fibre with the rejected dirt was achieved by injecting periodically the water into an underflow chamber to ensure clean water flow through the underflow aperture thus displacing fibre carrying water. Injection can be installed in this manner or arranged in a continuously operating system through the walls of the cone. In this case the exact position and design of the injection region is of considerable importance. The combination of position and design which gives optimum performance can only be determined by trial and error as noted by Kelsall and Holmes [84]. Figure 3.11 shows a technique which has been used successfully in certain cases. The given reason for the success in this case is that, this lowers the downward vertical velocity component. 


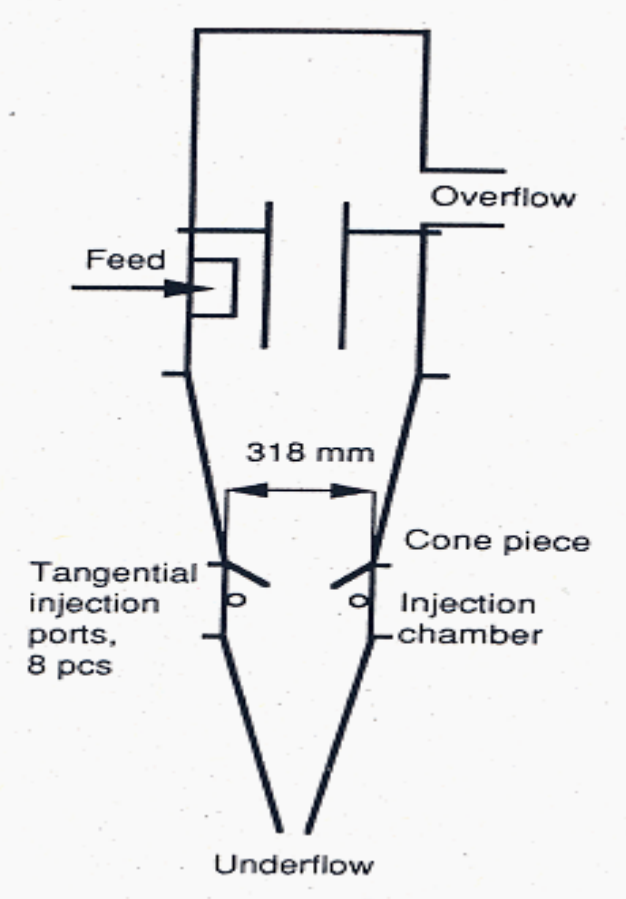

Fig. 3.11: Washing cyclone by Kelsall and Holmes [84].

Data illustrating the advantages of hydraulic water addition are given in the references $[83,84]$. Illustrative of these data are:

(1) A 3 inch cyclone operating on slurry of -20 mesh sand was used in two specific cases. It was found that about $48 \%$ and $43 \%$ of the minus $10 \mu \mathrm{m}$ material of the feed to the cyclone in these two cases respectively appeared in the underflow with normal operation (without water injection). These figures were reduced to $11.5 \%$ by water injection.

(2) A 24 inch cyclone operating in closed circuit with a ball mill treating $22 \mathrm{t} / \mathrm{h}$ of fresh feed was used in this case. The amount of minus 200 mesh in the underflow were decreased from 8.1 to 1.8 which reduced the percentage of product feed back to the mill (circulating load) from $350 \%$ to $160 \%$ of the mill feed.

(3) The slimes to overflow were increased from $83 \%$ to $99 \%$ in a 36 inch diameter hydrocyclone by the addition of the water at a rate around $30 \%$ of the feed flow. Slimes with the underflow solids were decreased from $0.6 \%$ to $0.03 \%$.

(4) The clay content of sand was reduced from $0.112 \%$ to $0.061 \%$ with the reduction being directly proportional to added water rate 
Dahlstrom [83] has successfully adopted this technique for desliming of mined product. Kelsall and Holmes [84] developed and demonstrated the first unit of the cyclone with the hydraulic water injection for the classification of sand. About 48 percent of the minus $10 \mu \mathrm{m}$ material in the feed reporting to underflow in normal operation was reduced to 11.5 percent after water injection.

Tests, using coal, have been carried out by Firth et al. [85] on a modified hydrocyclone with water injection. The system is commercially available from Krebs Engineers as the K H Cyclowash.

A further advantage of hydraulic water injection is that the risk of underflow blockage is minimized. The hydraulic water addition technique was not applied in general applications because each desliming problem requires an empirical approach to establish the best water addition conditions. Some generalisations can, however, be made. At higher feed rate, a large cone angle $\left(30^{\circ}\right)$ is preferable to achieve a better desliming efficiency with half the quantity of the hydraulic water required in the case of the using a small cone angle $\left(20^{\circ}\right)$. A vortex finder of greater length than that used for the conventional cyclone thickeners will minimize the loss of coarse solids to overflow due to the short circuit flow. Too long a vortex finder (greater than around $D_{c} / 2$ ) does, however, result in loss of sharpness and hence the optimum length is dependent on the feed size distribution. This is not an easy design variable to manipulate [8].

Krebs Engineers Company has recently developed a cyclone with water injection by inserting a truncated cone in the conical part of the hydrocyclone as shown in Fig. 3.12. This system is known as the $\mathrm{K} \mathrm{H}$ Cyclowash. The truncated cone is situated slightly in the conical part thus dividing the cyclone into two parts. In the upper section of the cyclone (above the truncated cone), primary classification of the feed material takes place. The classified coarse material enters the chamber below the truncated cone, where water is introduced tangentially. The injected water displaces the very fine particles to the overflow, thus achieving secondary classification.

Patil and Rao [86] have studied the impact of the truncated cone on the performance of a $100 \mathrm{~mm}$ Krebs model water-injection cyclone. The truncated cone was found to 
reduce the amount of particles less than particular size in the underflow. This size depends on the diameter of the truncated cone in conjunction with the inlet pressure. The technical details involving the parametric effects and interactions associated with the Cyclowash system remain limited.

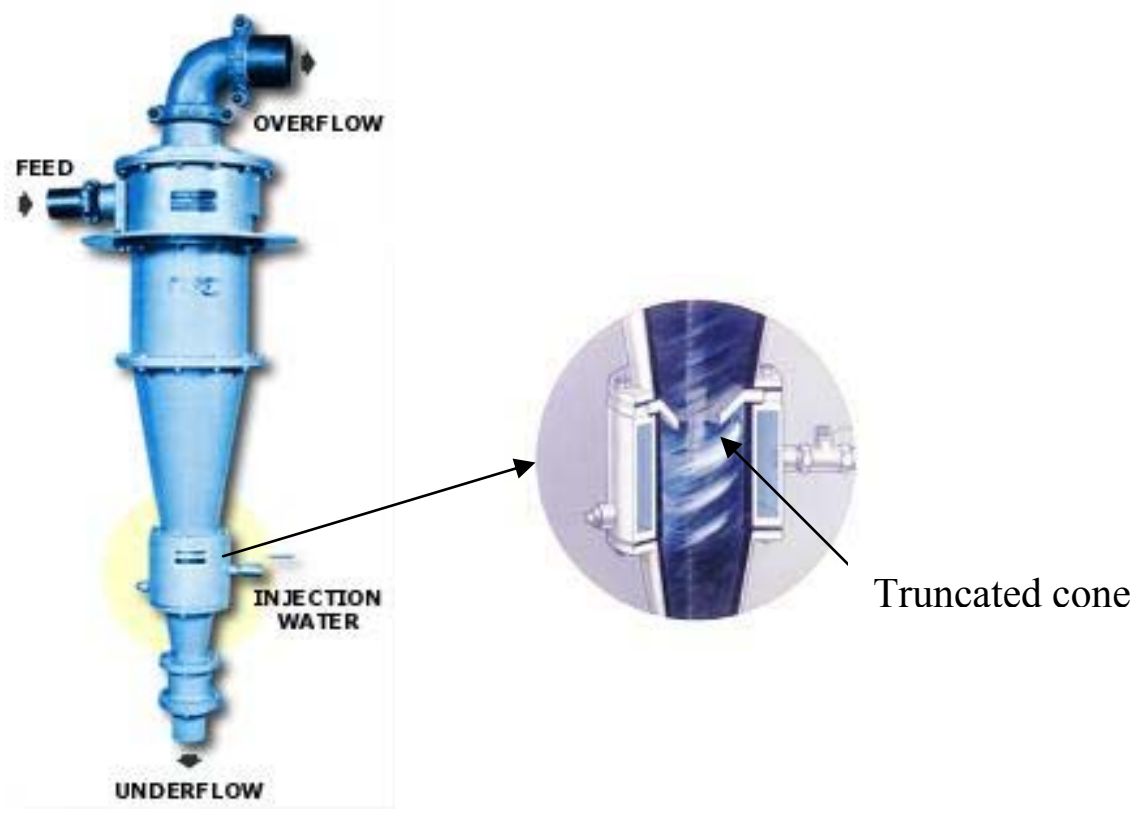

Fig. 3.12: Schematic diagram of Krebs CycloWash hydrocyclone.

In an attempt to investigate the Cyclowash efficiency, Honaker et al. [87] performed a test program based on a statistical design which involved the evaluation of the conventional cyclone and the Cyclowash parameters in a coal preparation plant located in western Kentucky, USA. The Cyclowash used in this investigation was $100 \mathrm{~mm}$ diameter having a cone angle of $10^{\circ}$ and inlet diameter of $3.8 \mathrm{~cm}$. The feed slurry contained about $4.5 \%$ solids by weight. About $1.5 \%$ of the material had a particle size greater $100 \mu \mathrm{m}$ and $65 \%$ less than $25 \mu \mathrm{m}$. The Cyclowash water rate was $38.5 \mathrm{l} / \mathrm{min}$ and the truncated cone diameter was $2.54 \mathrm{~cm}$. The actual separation curves indicated that the Cyclowash system had two main effects. Firstly, it increases the cut size $\mathrm{d}_{50}$ from about $20 \mu \mathrm{m}$ to $28 \mu \mathrm{m}$. Secondly, a substantial reduction in the amount of ultrafine by-pass has been achieved from nearly $18 \%$ to $8 \%$ as shown in Fig. 3.13. Considering that a large amount of the nominally $<150 \mu \mathrm{m}$ coal is below $25 \mu \mathrm{m}$ in particle size, the improvement is substantial. The test results were used to develop 
empirical models describing the percent by-pass to the underflow, corrected $\mathrm{d}_{50}$, and the imperfection value as a function of the operating and geometric parameters.

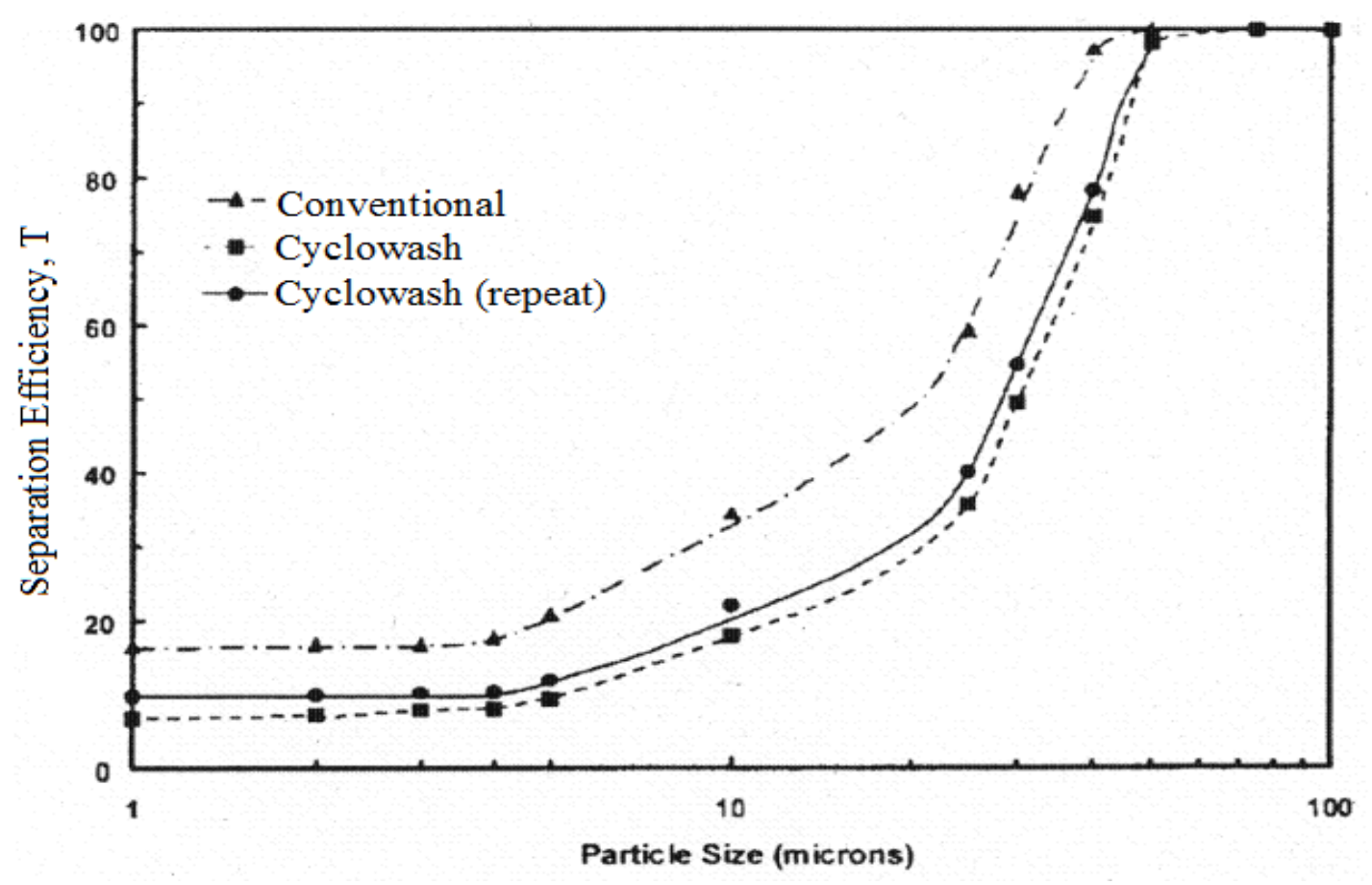

Fig. 3.13: Comparison of the actual partition curves obtained from conventional and Cyclowash cyclone units, after Honaker et al. [87].

Mohanty et al. [88] have tried to evaluate the efficiency of the gMax Cyclone with water injection system (Cyclowash) in the cleaning process of coal fine particles at 45 $\mu \mathrm{m}$ as compared with the conventional cyclones. It was found that using a $15 \mathrm{~cm}$ diameter gMax Cyclone, the performance is improved, where the oversize misplacement in the overflow was reduced from $18 \%$ in the conventional cyclone to $3 \%$ in g Max Cyclone. The undersize misplacement in the underflow was reduced from $54 \%$ in case of the conventional cyclone to $30 \%$ by water injection.

A classification studies were made by Udaya et al. [89] to recover liberated minerals from a ground lead-zinc ore. These studies were carried out using a $100 \mathrm{~mm}$ waterinjection cyclone. The effects of variables on the performance of water-injection cyclone were investigated. The results indicated that the entrapment of liberated heavy minerals of lead and zinc in the underflow product can be minimized with the help of water injection cyclone system. It also showed that the water injection rate 
has a complex interaction with the other variables like spigot opening, feed inlet pressure, and vortex finder opening. It was found that the cut size $\left(\mathrm{d}_{50}\right)$ increased gradually by increasing the water injection rate until it reached a value of $100 \%$ of the underflow water, and then increased steeply with a further increase in the water injection rate up to $160 \%$ of the underflow water rate. The results also indicated that the imperfection values decreased gently with the increase in water injection rate until it became about $100 \%$ of the underflow water and then decreased more steeply as shown in Fig. 3.14. They concluded that the optimum water injection rate is to be around $100 \%$ of the underflow water rate.

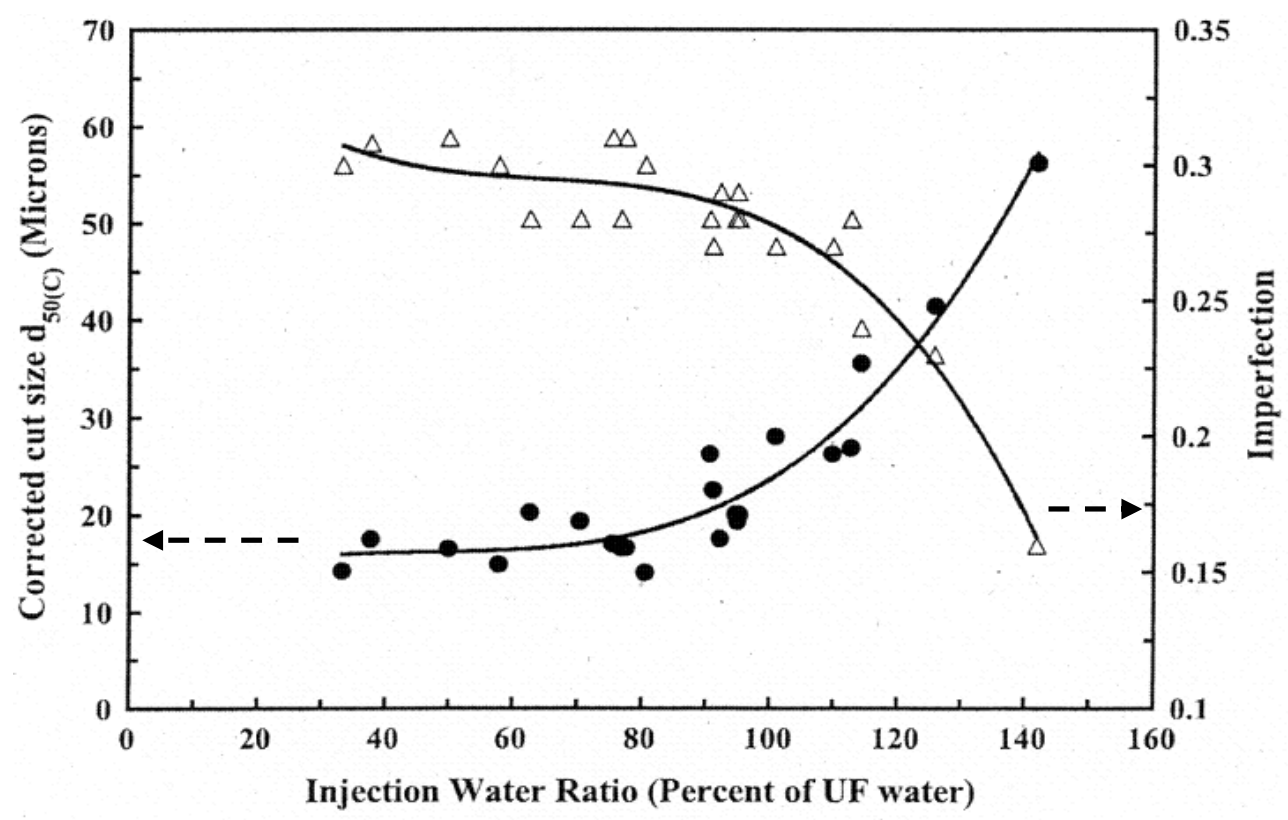

Fig. 3.14: Effect of injection water ratio on the corrected cut size and imperfection, after Udaya et al. [89].

\subsection{Conclusion}

It has been shown that considerable efforts have been made for improving the separation efficiency of hydrocyclone classifiers. The literature has highlighted some areas that need attention in improving the operation of the hydrocyclone unit and has shown that different modifications can be made to the hydrocyclone for use in a range of application. Different methods were tried to reduce the by-pass material to the underflow such as water injection cyclone, multi-stage cycloning, hydrocyclone 
control system, and in some cases special designs of hydrocyclone were used. It can be reported that most of these methods still have some limitations in the practical and industrial scales and have some disadvantages. Concerning the water injection, despite the success of the Cyclowash hydrocyclone in the minimisation of the fines in the underflow, there are some disadvantages of this hydrocyclone which can be summarised as follows:

- The large amount of water required for the Cyclowash to achieve the optimum separation (ranges from about $100 \%$ to $150 \%$ from the underflow water).

- The increase in the cut size $\left(\mathrm{d}_{50}\right)$ which accompanies the fines reduction

- Up to now, the Cyclowash is not tested for small cyclone size $(50 \mathrm{~mm})$ in which the injection effect becomes more complicated concerning the hydrodynamics and is more sensitive to the operating and constructional parameter.

- The truncated cone is subjected to wear which requires special maintenance and periodic replacement.

Finally, it can be concluded that most of the reviewed methods require more constructional areas, more capital and operating costs, more effort, more consumption of water and more plants and test rigs to suit the requirement of these methods. All of these reasons result in the motivation of the present work to minimise the disadvantages of these mentioned solutions. 


\section{Mathematical Modelling of the Injection System}

The mathematical analysis, which simulates the experimental results of the water injection in the conical part, was carried out by Dueck et al. [10]. The physical concept is a fluid flow model and the model of particle motion. The stationary concentration distributions result from the superposition of turbulent particle diffusion and particle settling. The particle interaction due to hindered settling, the effect of increasing the density and viscosity of the suspension, and the entrainment of the fine particle by settling coarse particles are included in the analysis. This mathematical treatment demonstrates the influence of the injection rate, injection velocity as well as the number of injection points and the location of the injection on the shape of the separation curve.

\subsection{Formulation of a Separation Model}

Fig. 4.1a shows a scheme of the model of turbulent cross flow classification introduced by Schubert and Neesse and modified by Dueck et al.[11],[59],[90,91]. The following simplifying assumptions are made:

- The main flow in the apparatus is positioned so that it crosses the direction of the separation field, i.e., the direction of the particle sedimentation with the settling velocity $V_{s, j}$ of the $\mathrm{j}$-th size fraction.

- The Reynolds numbers $\operatorname{Re}=U_{i} h / v$ indicate turbulent flow conditions. The turbulent particle transport is characterized by the turbulent diffusion coefficient $D_{t}$.

- At the end of the classifier the vertical size distributions are cut at height $h_{u}$. The underflow beyond the cut off (locus of zero axial velocity) should contain the coarse particles, and the overflow should have more fine particles.

- The model is completed by the water injection not far from the apparatus end and produces a current opposite to the settling direction with the velocity $V_{i n, 0}$. This counter current transports the fine particles to the overflow. 

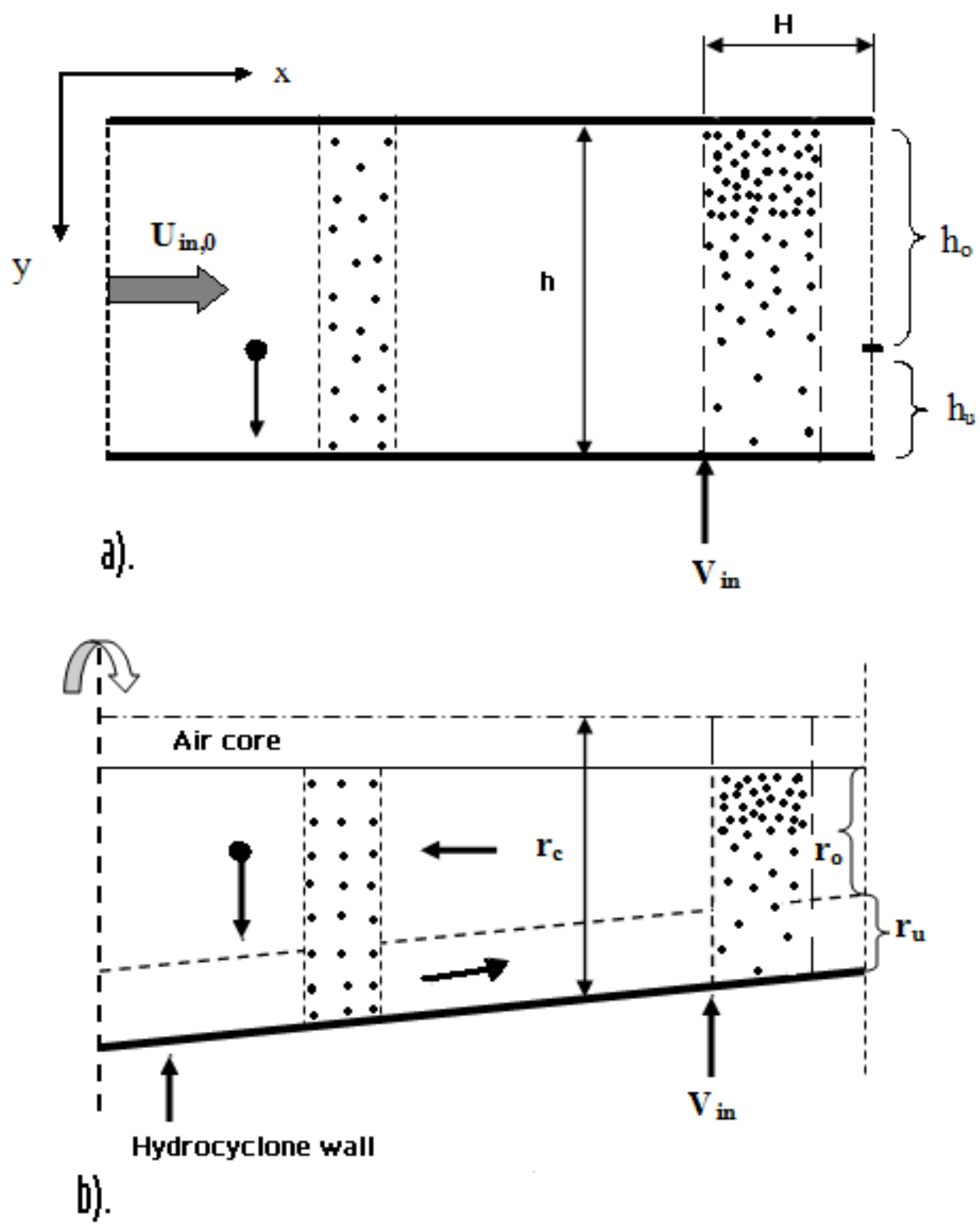

Fig. 4.1: Separation model of: a). Cross flow classifier, b). Hydrocyclone.

As seen in Fig. 4.1b, this model is applicable to the hydrocyclone. Here, in the axissymmetrical flow, settle the particles in a centrifugal field in the radial y-direction. The locus of zero axial velocity separates the outer and the inner vortex. In contrast to the cross flow model of Fig. 4.1a, the hydrocyclone overflow and underflow have opposite directions. The water injection is introduced tangentially with the main flow at a distance $\mathrm{H}$ from the under flow. This model considers that particles are noninertial: 


$$
\frac{d_{j}^{2}}{v} / \frac{d_{j}}{\left|V_{i n, y}-V_{s, j}\right|} \quad<1
$$

The transport equation, which describes the development of the local concentration $c_{j}$ of the $\mathrm{j}$-th size fraction (particle size diameter $d_{j}$ ) in the apparatus is:

$$
\frac{\partial U_{i}(x) c_{j}}{\partial x}+\frac{\partial}{\partial y}\left[\left(V_{s, j}+V_{i n, y}\right) c_{j}-D_{t} \frac{\partial c_{j}}{\partial y}\right]=0 .
$$

The boundary conditions are:

$$
\left(V_{s, j}+V_{i n, y}\right) c_{j}-D_{t} \frac{\partial c_{j}}{\partial y}=0 . \quad \text { for } y=h \text { and } y=0 .
$$

The condition at the entry is:

$$
\left.c_{j}\right|_{x=0}=c_{j, 0} .
$$

\subsection{Model of Jet Injection}

Under simplifying conditions, a soft injection can be modeled as jet flowing along the bottom of the apparatus. The component of the injection velocity along the jet describes a linear function of the coordinates across the main flow in the apparatus:

$$
\frac{V_{i n}(x, y)}{V_{i n, 0}}=\left\{\begin{array}{l}
0,0<x \leq L-H, \\
(-y / h) V_{r}, L-H<x \leq L
\end{array}\right.
$$

The volume conservation equation and Eq. (4.5) are used to determine the component of the velocity along the axis of the apparatus 


$$
\frac{U_{i}(x)}{U_{i, 0}}=\left\{\begin{array}{l}
1, \quad 0<x \leq L-H, \\
1+\left(V r / U_{i n l, 0}\right)(x-(L-H) / h), \quad L-H<x \leq L
\end{array}\right.
$$

$V_{r}$ can be approximated assuming that the injected water rate $I R$ flows through the cylinder area of a cylinder formed by the diameter $d_{i n}$ along the underflow wall. The injected water flow rate $I R(1 / \mathrm{min})$ can be described by:

$I R=\pi D_{u n} \pi d_{i n} V_{r}$.

Accordingly, the radial injection velocity can be expressed as follows:

$V_{r}=\frac{I R}{\pi^{2} d_{i n} D_{u n}}$

It should be noted that the radial injection velocity $\left(V_{r}\right)$ is directly proportional to the injection velocity $\left(V_{i n}\right)$, i.e. the higher is the injection velocity, the higher is the radial velocity.

\subsection{Definition of the Separation Curve}

As shown in Fig.1.4, the volume flux ratio of overflow and underflow (volume split parameter S) can be computed as follows:

$$
S=\frac{\int_{0}^{h_{o}} U_{i}(x, y) d y}{\int_{h_{o}}^{h} U_{i}(x, y) d y} .
$$


When there is no injection: $U_{i}(x)=U_{i, 0}$ and $S_{0}=\frac{h_{o}}{h_{u}}>1$.

The particle fluxes of the $\mathrm{j}$-size fraction $\mathrm{R}_{\mathrm{un}, \mathrm{j}}$ in the underflow and in the overflow $\mathrm{R}_{\mathrm{ov}, \mathrm{j}}$ are:

$$
R_{u, j}=\int_{h_{o}}^{h_{u}+h_{o}} U_{i}(L) c_{j}(L, y) d y,
$$

$R_{o, j}=\int_{0}^{h_{o}} U_{i}(L) c_{j}(L, y) d y$

The separation efficiency value $T\left(d_{j}\right)$ determines the portion of every size fraction that is discharged through the under flow can be given as follows:

$T\left(d_{j}\right)=\frac{R_{u, j}}{R_{u, j}+R_{o, j}}$.

The separation function is characterized usually by the following parameters:

- the $d_{50}$ which is the cut size with a $50 \%$ fractional recovery in the underflow

- the proportion $\mathrm{T}_{0}$ of the smallest particles that are misplaced in the coarse underflow. This value is important to characterize the washing effect of the water injection.

The separation function Eq. (4.10) can also be determined using the volume split $\mathrm{S}$ and the integral mean values of the concentrations in the underflow $c_{\mathrm{u}, j}$ and the overflow $c_{\mathrm{o}, j}$ :

$$
T\left(d_{j}\right)=\left(1+S \frac{c_{\mathrm{o}, j}}{c_{\mathrm{u}, j}}\right)^{-1}
$$


Finally, Eq. (4.11) approximates the ratio of the concentration mean values by using the concentrations on the bottom at $\mathrm{y}=0$ and on the upper end at $\mathrm{y}=\mathrm{h}$ (so called tapping model):

$$
T\left(d_{j}\right)=\left(1+S \frac{c_{o, j}(0)}{c_{u, j}(1)}\right)^{-1} .
$$

The concentrations in Eq. (4.12) are valid under the condition that at the discharge end of the apparatus, a stationary equilibrium is established (i.e., stable distributions of the particle concentration). In this case, Eq. (4.2) becomes

$$
\left(V_{s, j}+V_{r, y}\right) c_{j}-D_{t} \frac{d c_{j}}{d y}=0
$$

The solution of the differential Eq. (4.13) delivers the relative concentration distributions for the $\mathrm{j}$-th size fractions:

$$
\ln \frac{c_{j}(y)}{c_{j}(0)}=\frac{V_{s, j}}{D_{t}} y-\frac{V_{r}}{D_{t} h} \frac{y^{2}}{2} .
$$

Using Eq. (4.14) for the separation function of Eq. (4.12) results in:

$$
T\left(d_{j}\right)=\frac{1}{1+S \exp \left[-\frac{h}{D_{t}}\left(V_{s}\left(d_{j}\right)-0.5 V_{r}\right)\right]} .
$$

For $d_{j} \rightarrow 0$ follows:

$$
T(0)=\frac{1}{1+S \exp \left[\frac{h}{2 D_{t}} V_{r}\right]}
$$




\subsection{Model of Particle Settling in a Polydisperse Suspension}

There are numerous [7] experimental and theoretical results on the settling of dense suspensions. These studies focus on the settling behavior of polydisperse suspensions. It has been observed that the settling velocity of the fine particles increases in the presence of coarser fractions. The investigations of Deuck et al. [70] on the disturbed settling of dense suspensions were applied in the present model.

The polydisperse settling suspensions include the following interparticle effects:

1. Hindered Settling due to an increased effective density and viscosity of the fluid.

2. Counter flow of the displaced fluid caused by settling particles.

3. Entrainment of fine particles in the boundary layer range of coarse settling particles.

The complete equation for the settling velocity of $\mathrm{j}$-fraction particles is summarized $[92,93]$ as follows:

$V_{S, j}=\frac{V_{H, j}}{d_{j}^{2}}\left[d_{j}^{2}+g\left(c_{V}\right) f_{E}\left(d_{j}\right)-c_{V} \sum_{i=1}^{n}\left(d_{i}^{2}+g\left(c_{V}\right) f_{E}\left(d_{j}\right)\right) \frac{c_{j}}{c_{V}} \Delta d_{j}\right]$,

where, the first term of Eq. (4.17) is: Hindered settling

$V_{H, j}=V_{S t, j}\left(1-c_{V}\right)\left(1-c_{V} / 0.6\right)^{1.5}$

Here $V_{H, j}$ is the function describing the hindered settling due to higher values of viscosity and density, and $V_{S t, j}$ is the Stokes settling which is given by the following expression.

$V_{S t, j}=b \frac{g d_{j}^{2}}{18 \mu_{L}}\left(\rho_{S}-\rho_{L}\right)$

where $b$ - centrifugal Number (ratio of the centrifugal acceleration to the $g$ gravitational one), $\mu_{L}$ - liquid viscosity, $\rho_{S}$ - solid density, $\rho_{l}$ - liquid density. 
The second term of Eq. (4.17) is: Fine particle entrainment

$f_{E}\left(d_{j}\right)=\left(\sum_{i>j} \Delta m_{i} d_{i}^{6}\right)^{1 / 3}$

where,

$f_{E}\left(d_{j}\right)$ is the entraining function with $\Delta m_{i}=\frac{c_{i}}{c_{V}}=q\left(d_{i}\right) \Delta d_{i} . \quad$ The particle concentration of the $\mathrm{j}^{\text {th }}$ fraction are related to the total volume concentration of the solid phase $c_{v}$, which is related to the particle size distribution $q\left(d_{i}\right)=\frac{c_{i}}{\Delta d_{i} \sum c_{i}}$

The amount of the entrained coarse particles (i) for a given value of entrained fine particles $(\mathrm{j})$ can be obtained on the basis of the inequality derived for the bidisperse suspension [70]: $d_{i} \geq \beta d_{j}$. The theoretical value of $\beta$ confirmed by experiments is between 10 and 15 .

$g\left(c_{V}\right)=4.0 \cdot c_{V}^{2 / 3} \exp \left[-\left(\frac{c_{V}}{0.2}\right)^{3}\right]$

where, $g\left(c_{v}\right)$ is an empiric correction function from experiments [92] where the 3rd term accounts for the decreasing of the settling velocity due to the counter flow of displaced liquid. In summary, Eq. (4.13) shows that in a polydisperse suspension, the settling velocity of a particle depends not only on the total solids concentration $c_{v}$, but also on the particle size distribution. 


\section{Experimental Work}

\subsection{Material}

The feed material which was used through the present work was a synthetic mixture of kaolin and feldspar. It consists of about $70 \%$ by weight kaolin (fine) and $30 \%$ by weight feldspar (coarse). Fig. 5.1 shows the particle size distribution of kaolin/feldspar feed material.

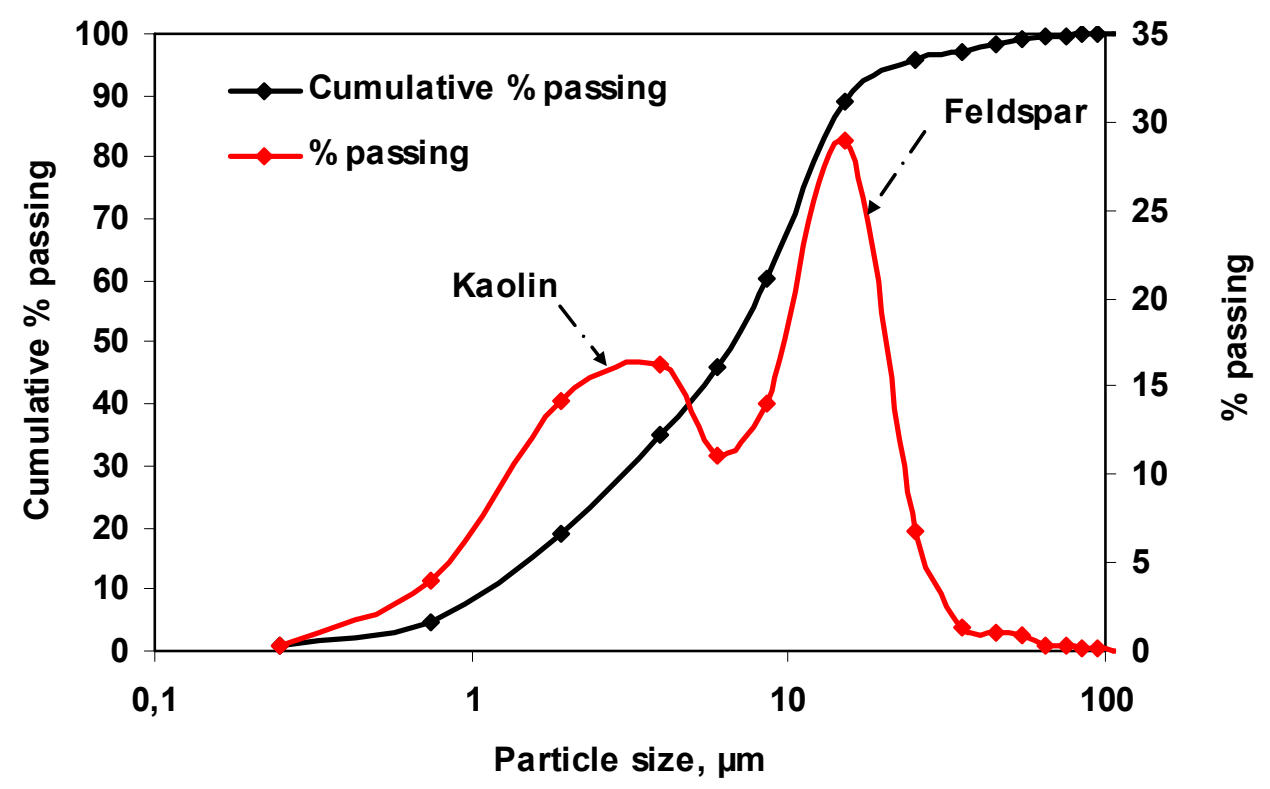

Fig. 5.1: Particle size distribution of kaolin/feldspar feed material.

\subsection{Apparatus and Test Rig}

\subsubsection{The $50 \mathrm{~mm}$ Water Injection Hydrocyclone}

The $50 \mathrm{~mm}$ diameter water injection hydrocyclone is a conventional cyclone with a modified conical part. This modification was commercially made at the Laboratory of Environmental Process Engineering and Recycling Institute, Freidrich-Alexander University, Erlangen, Germany. The lower section of the conical part, just above the apex, has a circular ring with injection openings distributed at equal distances on the periphery of the cone for water injection. This ring is connected with a control valve 
through which the water rate $(1 / \mathrm{m})$ can be adjusted to the desirable value. Figure 5.2 shows the geometry of the $50 \mathrm{~mm}$ water injection hydrocyclone.

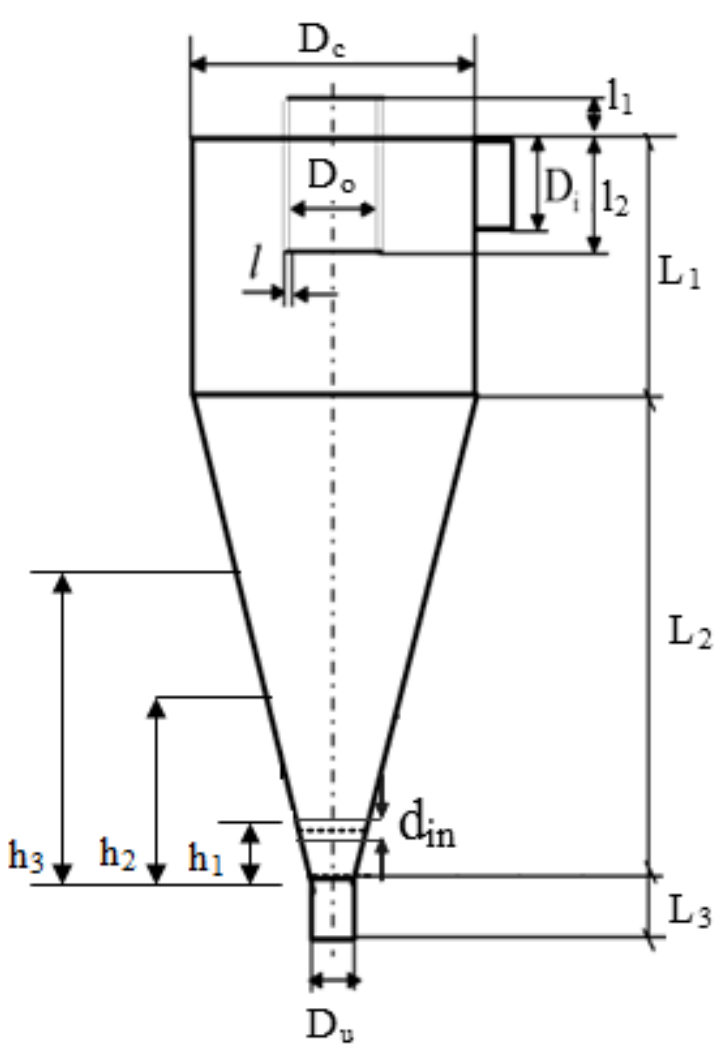

\begin{tabular}{|c|c|}
\hline Parameter & Dimension \\
\hline$D_{c}$ & $50 \mathrm{~mm}$ \\
\hline$D_{i}$ & $8 \times 18 \mathrm{~mm}$ \\
\hline$D_{o}$ & varied \\
\hline$D_{u}$ & varied \\
\hline$L_{1}$ & $130 \mathrm{~mm}$ \\
\hline$L_{2}$ & $350 \mathrm{~mm}$ \\
\hline$L_{3}$ & $135 \mathrm{~mm}$ \\
\hline$l_{1}+l_{2}$ & $79 \mathrm{~mm}$ \\
\hline$l_{2}$ & $40 \mathrm{~mm}$ \\
\hline$l$ & $8 \mathrm{~mm}$ \\
\hline$d_{\text {in }}$ & $1.5 ; 2.5 \mathrm{~mm}$ \\
\hline$h_{\text {in }}$ & $\begin{array}{l}\mathrm{h} 1=40 \mathrm{~mm} \\
\mathrm{~h} 2=90 \mathrm{~mm} \\
\mathrm{~h} 3=120 \mathrm{~mm}\end{array}$ \\
\end{tabular}

Fig. 5.2: Geometry of the $50 \mathrm{~mm}$ water injection hydrocyclone.

\subsubsection{Water Injection Assembly}

As can be seen from Fig. 5.3, the water injection assembly consists of an outer solid ring and an inner solid part of the cone with injection openings at equal distances along the perimeter. First, the water is injected radially in the outer ring to fill it. Then, the water is injected through the openings at the same time with the same velocity into the lower section of the conical part of the hydrocyclone. 

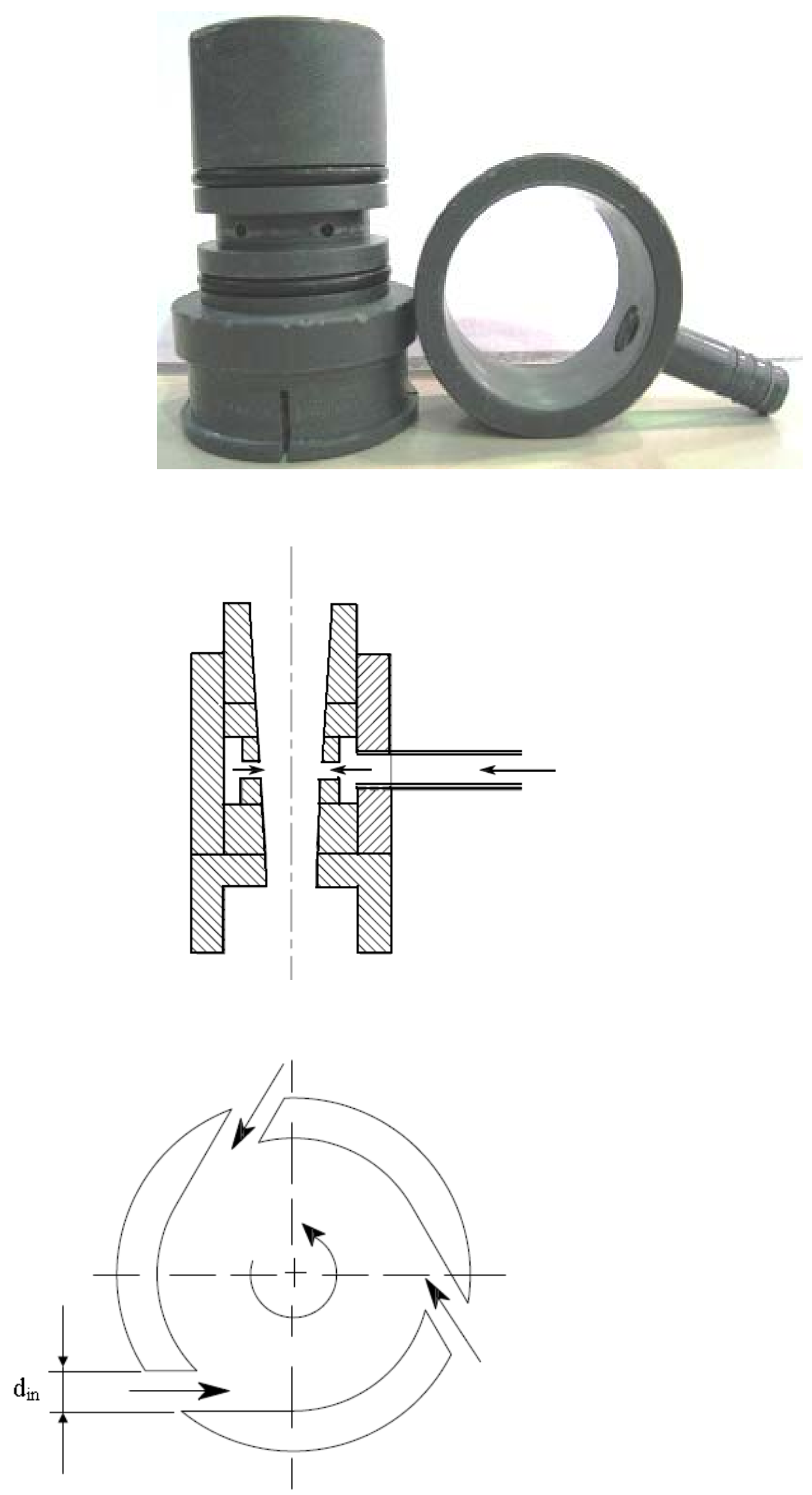

Fig. 5.3: Water injection assembly 
To investigate the effect of the injection direction, three different internal injection parts were designed. The only difference in these designs is the direction in which the water will be injected through the openings into the cone. These injection parts were designed to inject the water in the following directions:

a- In a tangential direction with the same swirling motion of the flow inside the hydrocyclone.

b- In a tangential direction with an opposite direction to the swirling motion of the flow inside the hydrocyclone.

c- In a radial direction towards the hydrocyclone axis.

Fig. 5.4 shows the different injection directions of the water through the different injection parts.

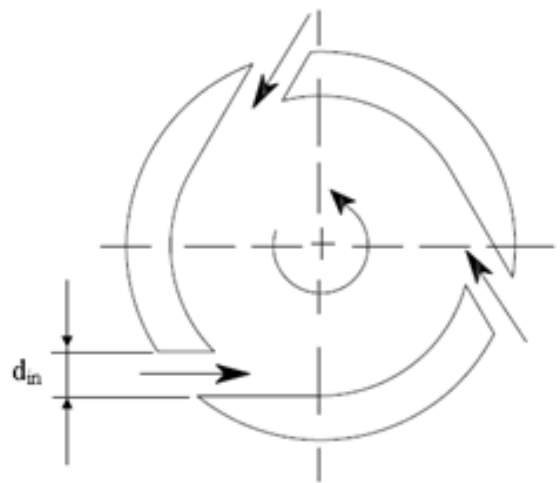

(d)

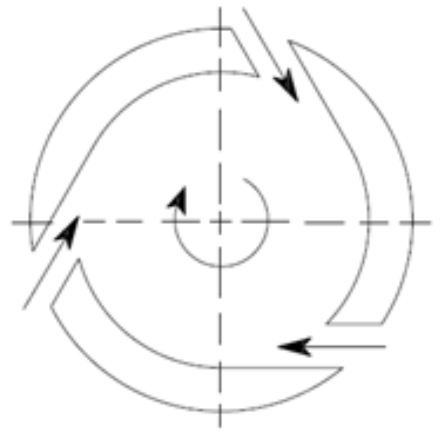

(b)

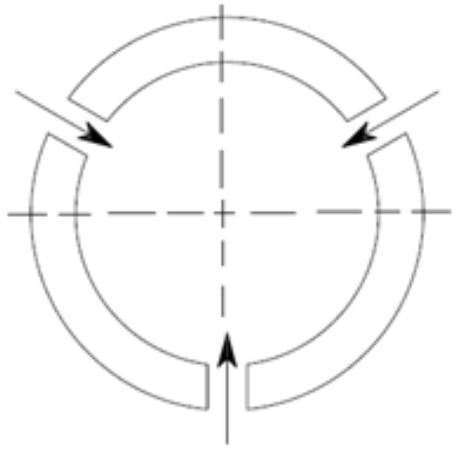

(c)

Fig. 5.4: Different injection rings with different injection directions used for the 50 $\mathrm{mm}$ water injection hydrocyclone;

(a) Tangential direction with the suspension flow direction

(b) Tangential direction opposite to the suspension flow direction

(c) Radial direction towards the hydrocyclone axis.

To investigate the effect of the injection openings number, two identical injection parts were designed with different number of injection openings of 3 and 5 openings. These rings are shown in Fig. 5.5. 


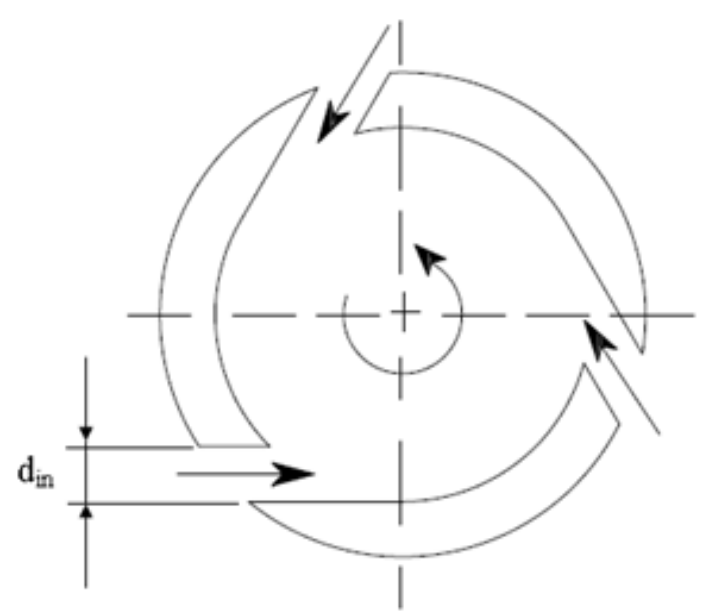

(d)

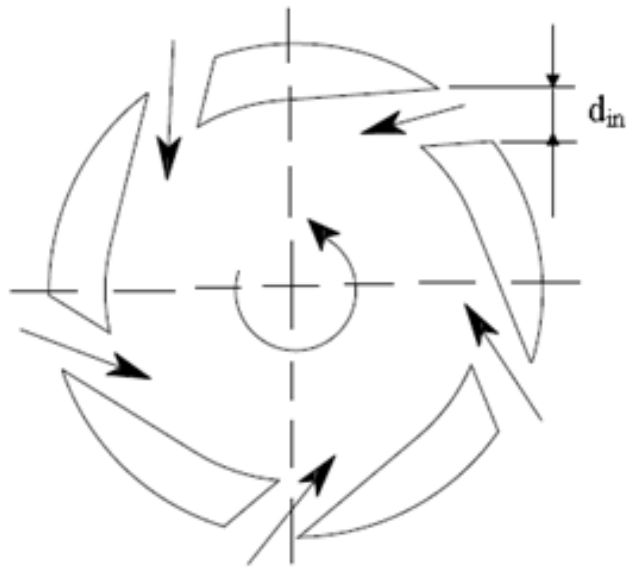

(b)

Fig. 5.5: Water injection rings with different openings number; (a) three openings (b) five openings.

It was also important to investigate the effect of the injection opening diameter. Therefore, another two identical parts with 3 injection openings with $1.5 \mathrm{~mm}$ and 2.5 $\mathrm{mm}$ injection opening diameter were designed.

To investigate the effect of the height of the injection point from the upper end of the apex level, three injection parts were designed to be located at different heights called h1 (the lowest height), h2 (the intermediate height), and h3 (the highest level from the apex). Figure 5.6 shows the $50 \mathrm{~mm}$ water injection hydrocyclone with the different manufactured parts of water injection at different heights from the cyclone apex used. 


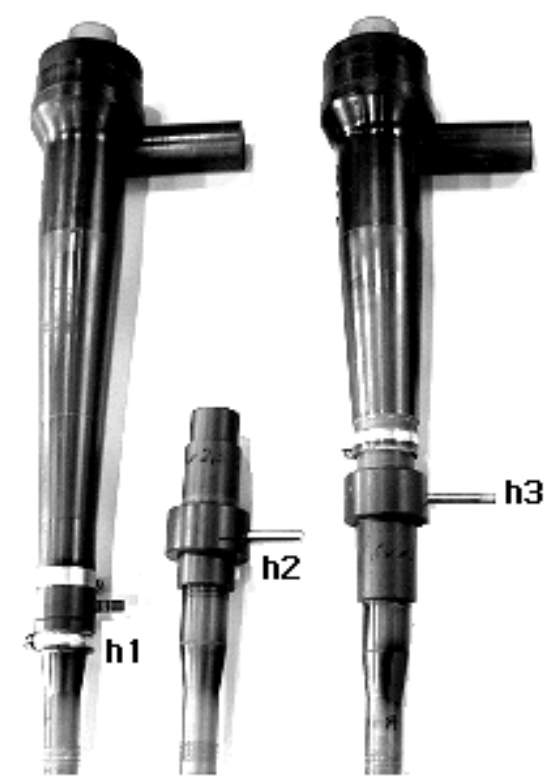

Fig. 5.6: The water injection hydrocyclone with different injection heights.

\subsubsection{The Water Injection Hydrocyclone Test Rig}

The test rig used in the water injection experimental work consists of a $50 \mathrm{~mm}$ diameter water injection hydrocyclone positioned vertically above a feed tank of 100 1. The pump is connected to the hydrocyclone feed inlet. A by-pass pipe with a control valve was connected to the outlet line to obtain the desired pressure drop. A pressure gauge was fitted near the feed inlet to indicate the pressure drop. A water control valve was installed through the water supply pipe to adjust the desired amount of the injected water. A digital manometer was fixed near the water injection valve to indicate the water injection rate in $1 / \mathrm{min}$. Figure 5.7 shows the $50 \mathrm{~mm}$ water injection cyclone test rig. 

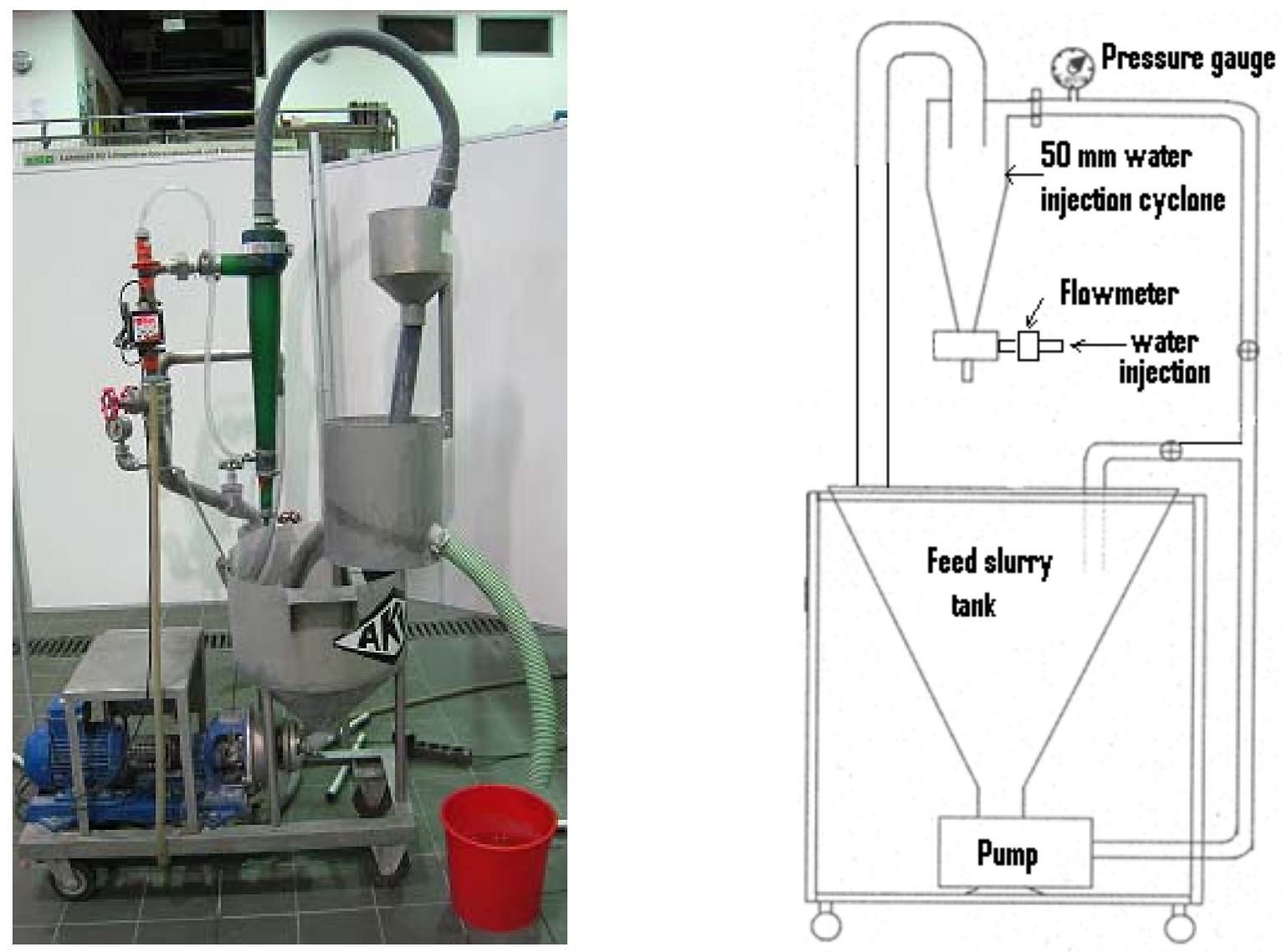

Fig. 5.7: The water injection hydrocyclone test rig.

\subsubsection{Operating the Water Injection Hydrocyclone Test Rig}

In the test rig of the water injection hydrocyclone, the water was injected by controlling the valve to be opened with the desired water rate through the digital flow meter. The flowmeter can supply water flow rate starting from $0.01 \mathrm{l} / \mathrm{min}$ up to 10 $1 / \mathrm{min}$. The test rig was first operated without water injection (normal operation) and then the water was injected at different rates to study the effect of the wash water on the hydrodynamics inside the cyclone and later on the separation efficiency of the fine particles.

\section{3 Test Program and Procedure}

\subsubsection{The Water Injection Hydrocyclone Test Program}


Different designing and operating parameters were tried in the water injection process using the water injection hydrocyclone. First, the experiments were carried out using only water as feed to study the effect of the water injection on the distribution of the water through the overflow and underflow openings. The test program of the water experiments is shown in Table 5.1. After that, the main test program of the experiments using a suspension was carried out as shown in Table 5.2.

Table 5.1: Test program of water only experiments

\begin{tabular}{|c|c|}
\hline Parameter & Value \\
\hline Water injection rate (1/min) & $1,2,3,4,5,6,7$ \\
\hline Feed pressure, (bar) & $0.6,0.8,1,1.2,1.6,2$ \\
\hline Number of injection openings & 3,5 \\
\hline
\end{tabular}

Table 5.2: Test program of suspension experiments

\begin{tabular}{|c|c|}
\hline Parameter & State \\
\hline Overflow diameter, $\mathrm{D}_{\mathrm{o}}, \mathrm{mm}$ & $16,18,20,22$ \\
\hline Apex diameter, $\mathrm{D}_{\mathrm{u}}, \mathrm{mm}$ & $4.2,6,8,10,13$ \\
\hline Water injection rate, $1 / \mathrm{min}$ & $1,2,3,4,5,5.7$ \\
\hline Feed pressure, bar & $0.8-1-1.5-2$ \\
\hline Feed solid content, g/l & $50,100,150,220$ \\
\hline Number of injection openings & 3 and 5 \\
\hline Injection opening diameter, $\mathrm{mm}$ & 1.5 and 2.5 \\
\hline Inner vortex finder length, $\mathrm{mm}$ & $40,80,120$ \\
\hline $\begin{array}{l}\text { Injection height from the upper end } \\
\text { of the apex, } \mathrm{mm}\end{array}$ & $40,80,120$ \\
\hline Injection direction & $\begin{array}{l}\text { 1-Tangential with the suspension direction } \\
\text { 2- Tangential against the suspension direction } \\
\text { 3- Radial towards the cyclone axis }\end{array}$ \\
\hline
\end{tabular}




\subsubsection{Procedure of Water Injection Experiments}

The water injection procedure for the experiments carried out on suspensions using the water injection hydrocyclone is shown in Fig. 5.8. A suspension of feldspar/kaolin and water was prepared with a solid content about $220 \mathrm{~g} / \mathrm{l}$ in the sump of the test rig. In each test the pre selected parameters (overflow opening diameter, underflow opening diameter, and injection ring type) are fit. The test rig is operated and the suspension is fed to the hydrocyclone. The feed pressure is then adjusted to the desired value. Then the closed circuit of the suspension is completed. The overflow, underflow, and feed discharges create the agitation system for the suspension to be homogenous in the tank. After the steady state conditions are reached, samples from overflow and underflow discharges were taken before the water injection. After that, the water valve was opened to inject the desired amount of water. Then, representative samples from the overflow and underflow discharges at every wash water rate are taken to further measurements and analysis. At the end of the experiment, the water valve is closed, the feed solids are discharged to a container box, the pressure being lowered down to zero value and then the test rig is switched off. 


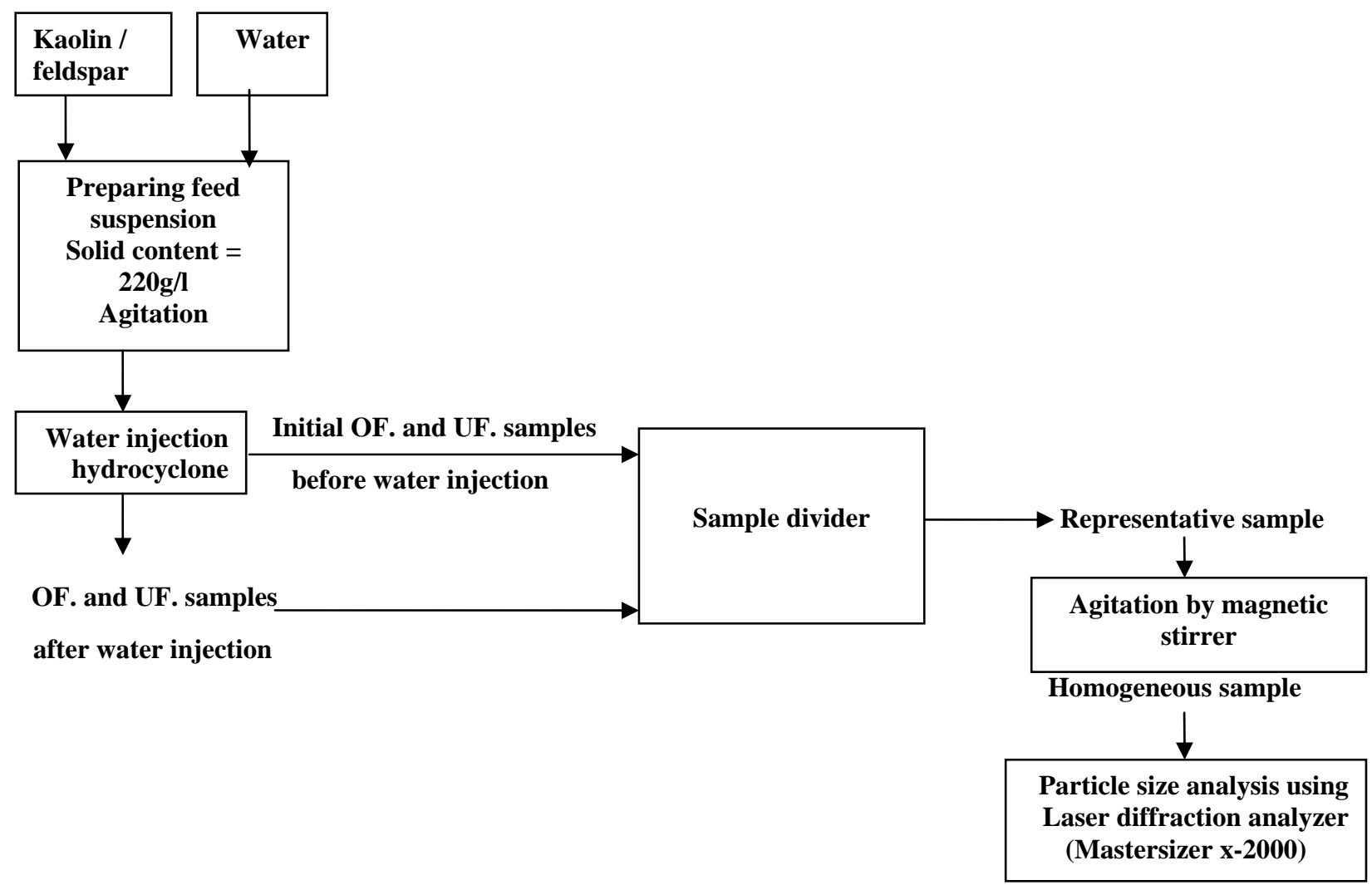

Fig. 5.8: Procedure of water injection experiments.

\subsection{Particle Size Analysis}

The particle size analysis was carried out using the laser scattering light analyzer (Mastersizer $X$-2000) as shown in Fig. 5.9.

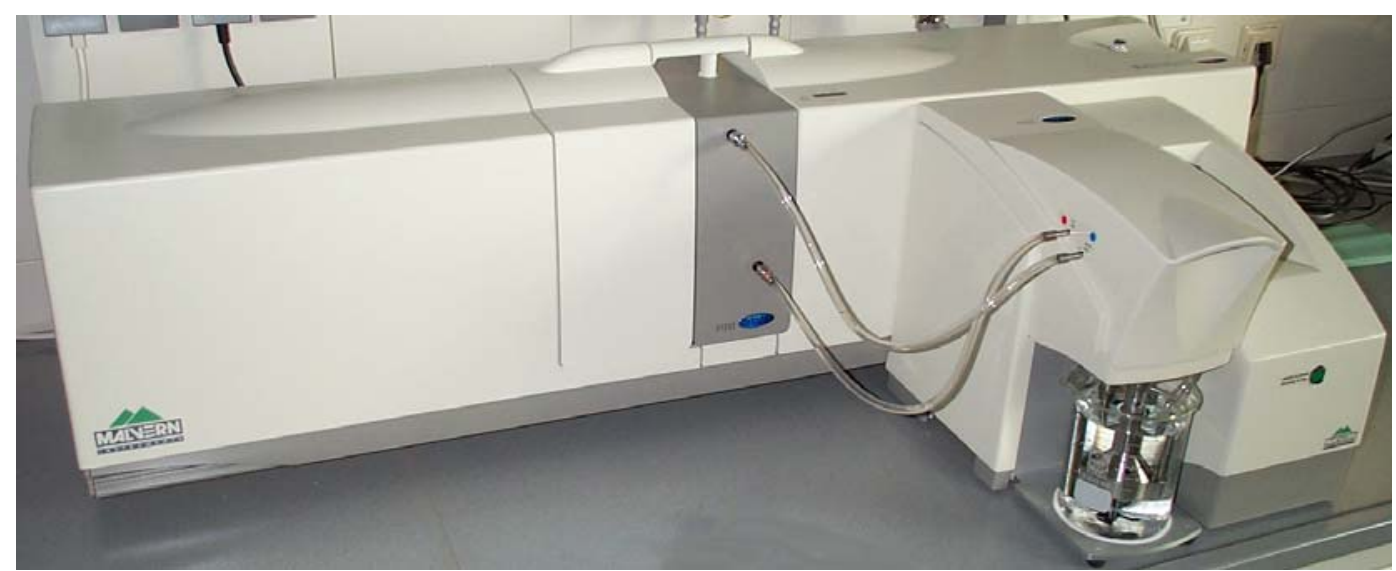

Fig.5.9: Laser diffraction analyzer (Mastersizer $X$-2000). 
The fundamental principle of the laser diffraction is shown in Fig. 5.10. The particles pass through a collimated laser beam. They will scatter the light at different angles which are inversely proportional to their sizes.

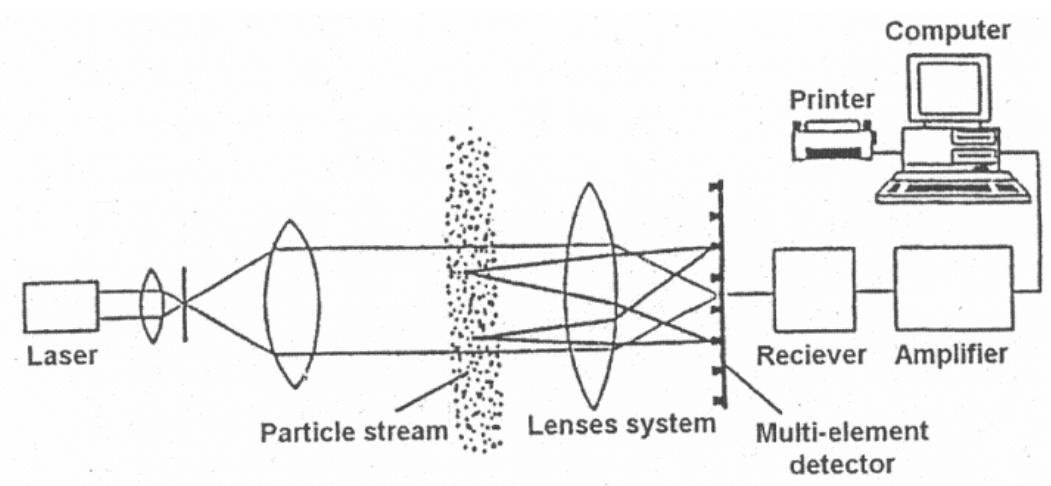

Fig.5.10: A schematic of the laser diffraction fundamental principle.

The laser scattering light analyzer has many advantages which can be summarized by the following:

- Just a very small sample (some drops) is sufficient to determine the particle size distribution.

- The measurements are reproducible (despite the small size of the measured particles).

- The measurements are very fast (few seconds).

- Ability of measuring a wide size range.

- The sample is not destructed (can be recovered for further treatment).

\subsubsection{Preparation of the Sample for Analysis}

To obtain a representative sample suitable for the Mastersizer instrument, the sample is first divided for eight representative samples using the wet sample divider shown in Fig. 5.11. Then one of these eight samples is taken and agitated well using a magnetic stirrer shown in Fig. 5.12. 


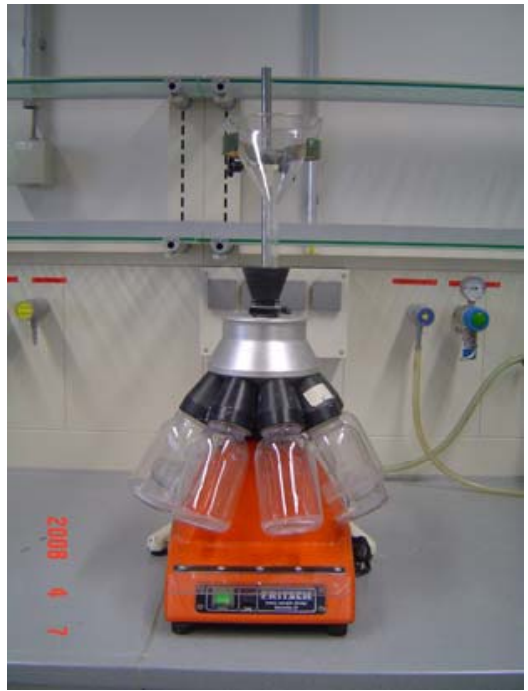

Fig.5.11: The sample divider.

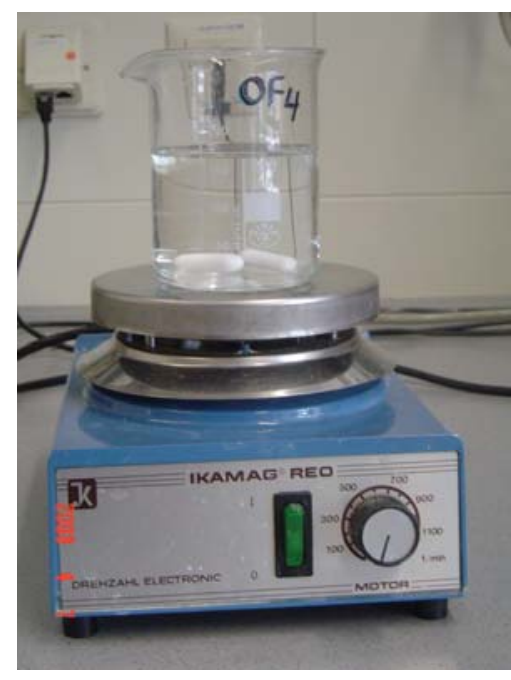

Fig.5.12: The Magnetic

stirrer.

\subsubsection{Sample Analysis}

The Mastersizer instrument should be run at least 20 minutes before starting the sample analysis so that the laser intensity reaches the proper range. Then the glass bottle should be filled with distilled water $(600 \mathrm{ml})$ and then the pump is turned on. After that, small drops of the representative sample are taking while the sample is being agitated, and injected into the sampler container using a special injecting tube. Then the ultrasonic is to be turned on to ensure that the particles are dispersed and not agglomerated anymore to obtain a real size distribution of the sample. The analysis process takes about 3 minutes to be done and the particle size distribution curve and table to be obtained. 


\section{Data Treatment and Error Estimation}

\subsection{Data Treatment for Separation Curve Determination}

The separation curve determination is based on the experimental data obtained from the measurement of the overflow, underflow, and feed samples. The direct measurements of weight, volume and particle size distribution of these samples can not be used directly to construct the separation curve. These data must be treated to obtain the solid content and the mass recoveries of the overflow and underflow streams together with the selection of the particle size range required for good illustration of the separation curve. The solid content of the overflow and underflow streams can be determined using Eq. (6.1).

$C=\rho_{S} \frac{\frac{m_{\text {sus }}}{V_{\text {sus }}}-\rho_{l}}{\rho_{S}-\rho_{l}}$,

where, $\rho_{S}$ is the solid density, $m_{\text {sus }}$ is the suspension mass, $V_{\text {sus }}$ is the suspension volume, $\rho_{l}$ is the liquid density.

Knowing the solid content, the volume of the sample, and the time of the sample, then the solids flow rate of overflow and underflow can be easily determined using Eq. (6.2) and Eq. (6.3) respectively:

$U=V_{u} C_{u}$

$O=V_{o} C_{o}$

where, $U$ and $O$ are the solid flow rates of the underflow and overflow products respectively, $V_{u}$ and $V_{o}$ are the suspension volume flow rate $(1 / \mathrm{h})$ of underflow and overflow respectively, and $C_{u}$ and $C_{o}$ are the solid content $(\mathrm{g} / \mathrm{l})$ in the underflow and overflow. 
Then for every particle size $(d)$ the separation efficiency $T(d)$ can be calculated using Eq. (1.1) which can be rewritten as follows :

$$
T(d)=\frac{U^{*} u(d)}{F^{*} e(d)}=\frac{U^{*} u(d)}{U^{*} u(d)+O^{*} o(d)},
$$

where, $U, O$ and $F$ are the solids flow rates in underflow, overflow, and feed respectively and $u(d), \mathrm{o}(\mathrm{d})$, and $\boldsymbol{e}(d)$ are the weight fractions of particle size $(d)$ in the underflow, overflow and feed streams respectively.

\subsection{Experimental Error Estimation}

In the present work, compromising between the work to be done and the accuracy of the research results was the main concept which the experimental procedure was based on. Accordingly, it would be important to report the level of error or uncertainty associated with the different parameters measurements which are used in drawing the separation curve of the hydrocyclone. Errors may be divided roughly into two categories: Systematic error and statistical (random) error.

\subsubsection{Systematic Error Sources of the Present Work.}

Systematic errors are difficult to detect and can not be analyzed statistically. This type of error can be reduced but can not be completely eliminated. The systematic errors sources which could be arose during the present work are mainly due to the sampling process. The sampling process involves the method of taking the sample, the determination procedure of the volume flow, solids flow, and the particle size flow of the feed, the overflow, and the underflow streams.

\subsubsection{Sampling Procedure}

The accurate sampling procedure to make a mass balance of the hydrocyclone separation process is to take two timed samples from both overflow and underflow streams simultaneously at the same time. 
In the present work, it was not easy to take the overflow and underflow samples at the same time accurately by the same operator especially in the case of injection. Using two operators for doing such sampling could increase the personal error percentage accompanied with the sampling process which could be more than the systematic error value itself. So, the used sampling procedure in the present work was to take the two samples immediately after each other (the overflow sample followed by the underflow sample). This is justified in a closed circuit test rig under stable conditions. In the present work, both overflow and underflow samples were collected directly from the whole overflow stream and from the underflow discharge to assure the representation of the samples.

\subsubsection{Volume Flow}

The volume flow rate of any stream can be determined by taking a sample from the whole stream for a certain period of time. In the present work, it was possible to determine the volume flow of the streams directly by measurements not by volume balance calculation. This was done by taking direct samples from the whole overflow stream, the whole underflow discharge and the whole feed flow from the by-pass pipe. By using this direct method of volume determination, it was possible to avoid the error which is produced if the volume was determined using the mass balance calculations.

Another important source for the sampling errors is the volume of the sample to be taken. The larger the sample; the lower is the percentage error. In the present work, it was not possible to take large samples. This is due to the small volume of the tank of the test rig (100 1). The tank was filled with about 501 of suspension and the rest of the volume was left for the expected volume of the injected water. So, sample size may affect the feed characteristics such as the size distribution, the solid content, and the flow rate. Also, the static head above the pump inlet becomes sensitive to the decrease in the suspension volume. Therefore, large volume samples may affect the feed pressure.

To minimise the error arises from the sample volume, five repetitions were carried out to optimise the volume of the sample which could be representative of the flow 
without changing the feed characteristics. Hence, for all samples, approximately the same volume was taken. Figure 6.1 and Fig. 6.2 show the effect of the sample volume on the corresponding density of the overflow and underflow suspensions at the same conditions. From these figures, it can be shown that at low sample volume, the density is not stable until a certain sample volume of about 0.71 in the case of the overflow sample and about 0.61 in the case of the underflow samples. At these values the density becomes relatively stable and further increase in the sample volume does not affect the determined density any more. Accordingly, the optimum sample volume which represents the overflow and underflow samples well should not be less than these values to obtain accurate results.

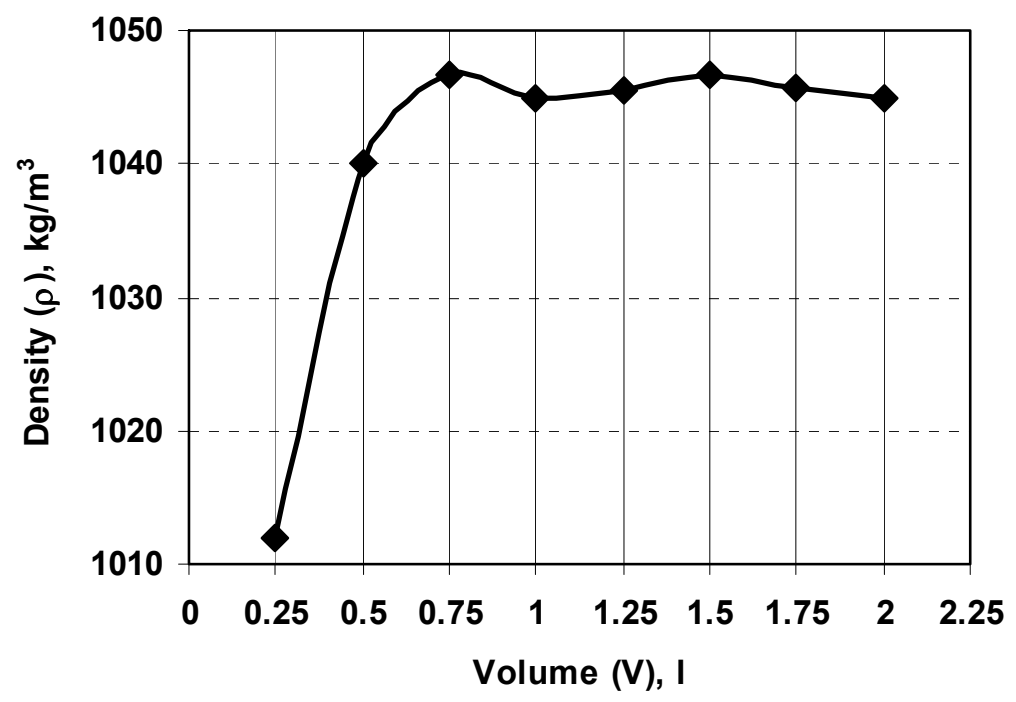

Fig.6.1: Effect of the sample volume on the overflow density.

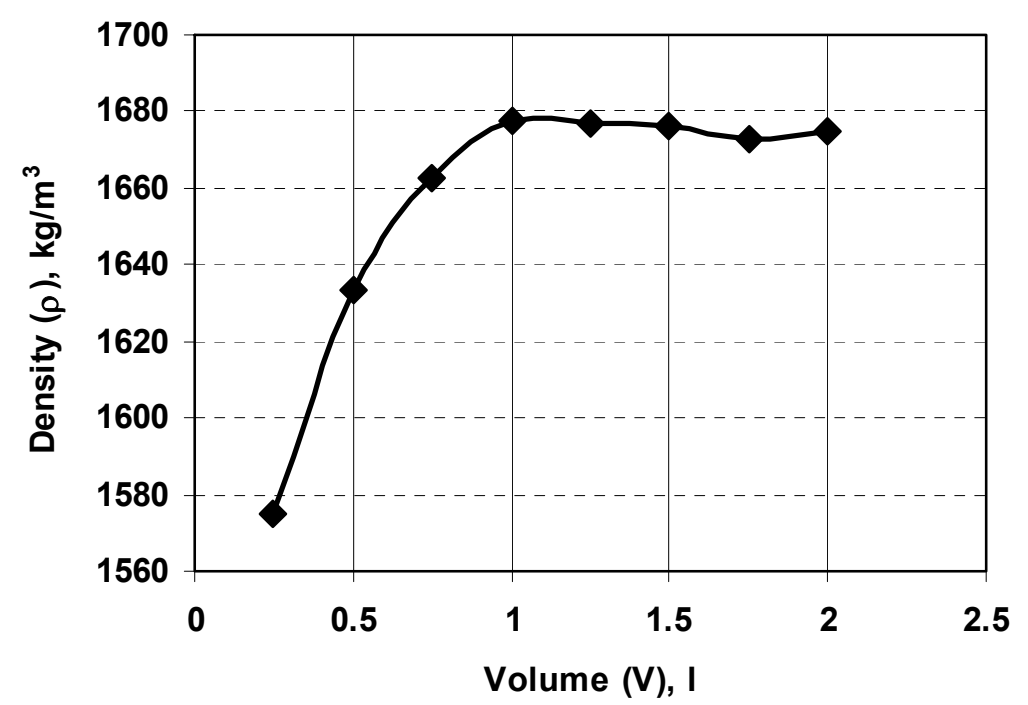

Fig.6.2: Effect of the sample volume on the underflow density. 


\subsubsection{Solids Flow}

The accurate method for determining the solid content of any suspension is to take a representative sample from the suspension, filter, dry, and weigh the dried solids. On the other side, using this method, the loses of fines through the filtration media, (prior to drying), the effort of filtration and drying processes, the time needed for handling large number of samples may presents some disadvantages. Also, the accumulative error of these processes could be of a high value and affects the results accuracy. In an attempt to reduce the effort and handling steps of the samples without affecting the accuracy of the results, another method for the determination of the solid content was used. This method is based on weighing accurately the determined volume of the suspension so that the solids content can be calculated using Eq. (6.1).

The error which arises from this method occurs due to the inaccuracy in the determination of the suspension density which depends on the reading values of both weight and volume of the sample. The error of the volume readings (which is usually approximated value) due to the scale of the graduated bottle is considered one of the potential sources of error. This can not be totally avoided, but can also be reduced by unifying all the used bottles with the same scale. The bottles should be put on the same flat horizontal level and the readings are to be taken from constant horizontal level. Also, the samples' volume should be as the same as possible for all of the different samples. To check the accuracy of this method, the solid content was determined by using the two different methods (dry and weigh method, and formula method). The results were compared as can be shown in Fig. 6.3. 


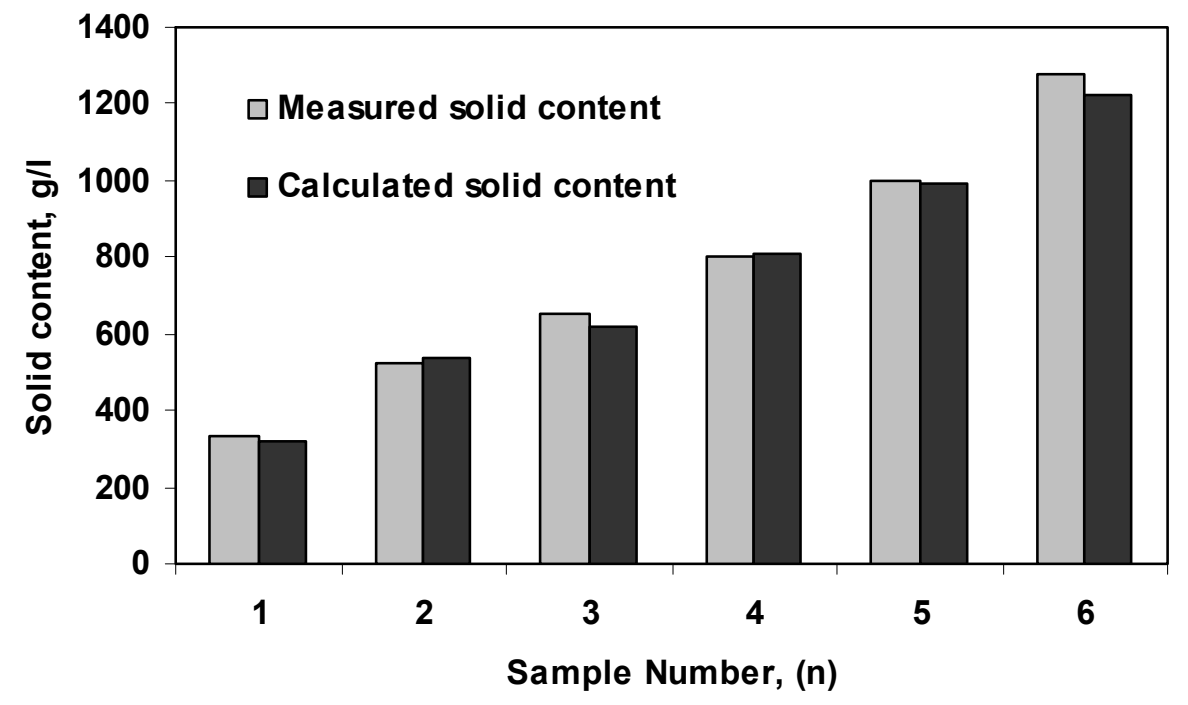

Fig. 6.3: Comparison of the solid content of different samples determined by the formula method and the dry and weigh method.

The correlation between the solid content values obtained from both methods is presented in Fig. 6.4.

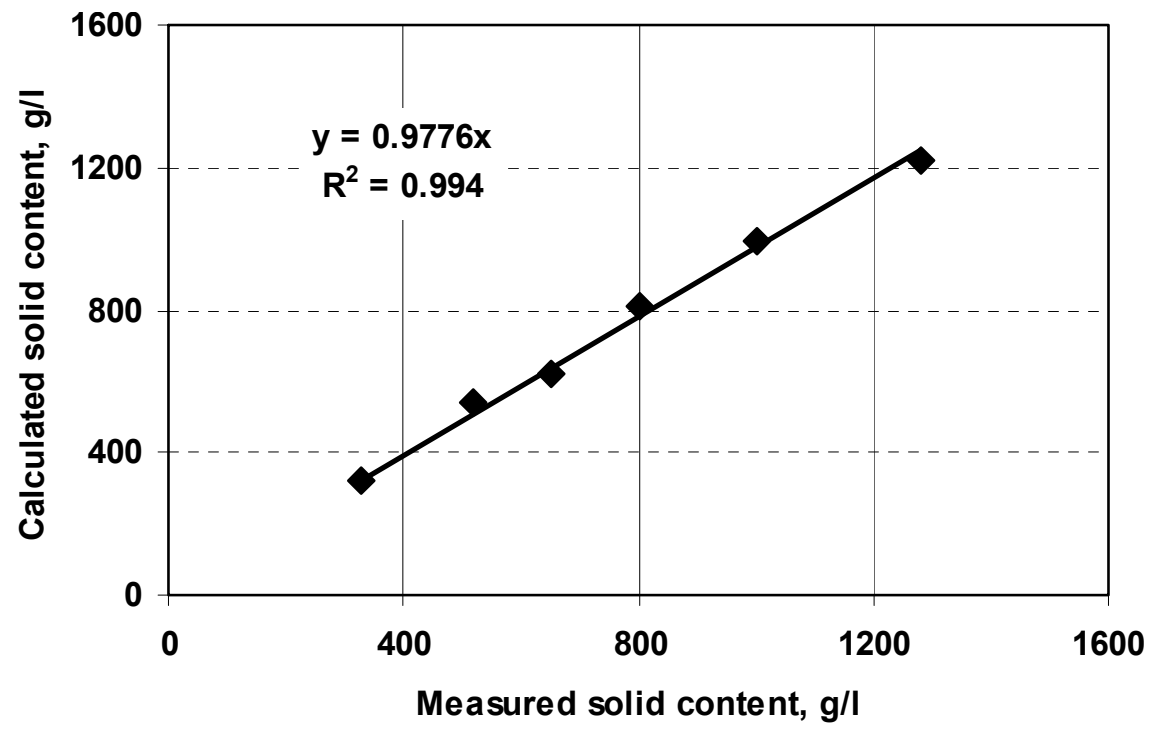

Fig. 6.4: Correlation between calculated and measured solid content (g/l).

It should be noted that, this method is not recommended to be used at low feed solid content (less than $50 \mathrm{~g} / \mathrm{l}$ ) because it may lead to negative values of the suspension density. In the present work, the feed solid content was more than $200 \mathrm{~g} / 1$ in all experiments; therefore, it was safe to use this calculation method to determine the solid content accurately. 


\subsubsection{Particle Size Analysis}

As it was previously mentioned, the volume of the sample is of a great importance and could affect the accuracy of the results. For an accurate particle size analysis, the sample should be representatively taken. The normal procedure is to use one sample from each stream at each operating condition. This sample is used for both the mass balance (density and solid content determination) and for the analysis process. This procedure was not suitable in the case of large volume samples because the handling of such samples is not easy. On the other hand, the division process of the large sample, starting from one or two litres down to some drops which are required for the analysis, may increase the systematic error especially for the coarse suspension. Accordingly, two samples of different volumes for the same stream at the same operating condition were taken. One large sample about 11 was taken for the mass balance and another small sample about $200 \mathrm{ml}$ was taken for the size analysis. With such a small volume sample, the error which is produced from the division process of the sample can be reduced specially for the underflow samples. Figures 6.5 and 6.6 show the size distribution of eight samples produced by the sample divider in the case of large volume samples (more than 11 ) and of small volume sample (about $200 \mathrm{ml}$ ) at the same operating conditions. As can be seen from these figures, the difference between the size distributions in the case of the big volume sample is more than the difference in the size distributions in case of the small volume sample. This supports strongly the use of a large volume sample for the mass balance calculations and another small volume sample for size analysis. 


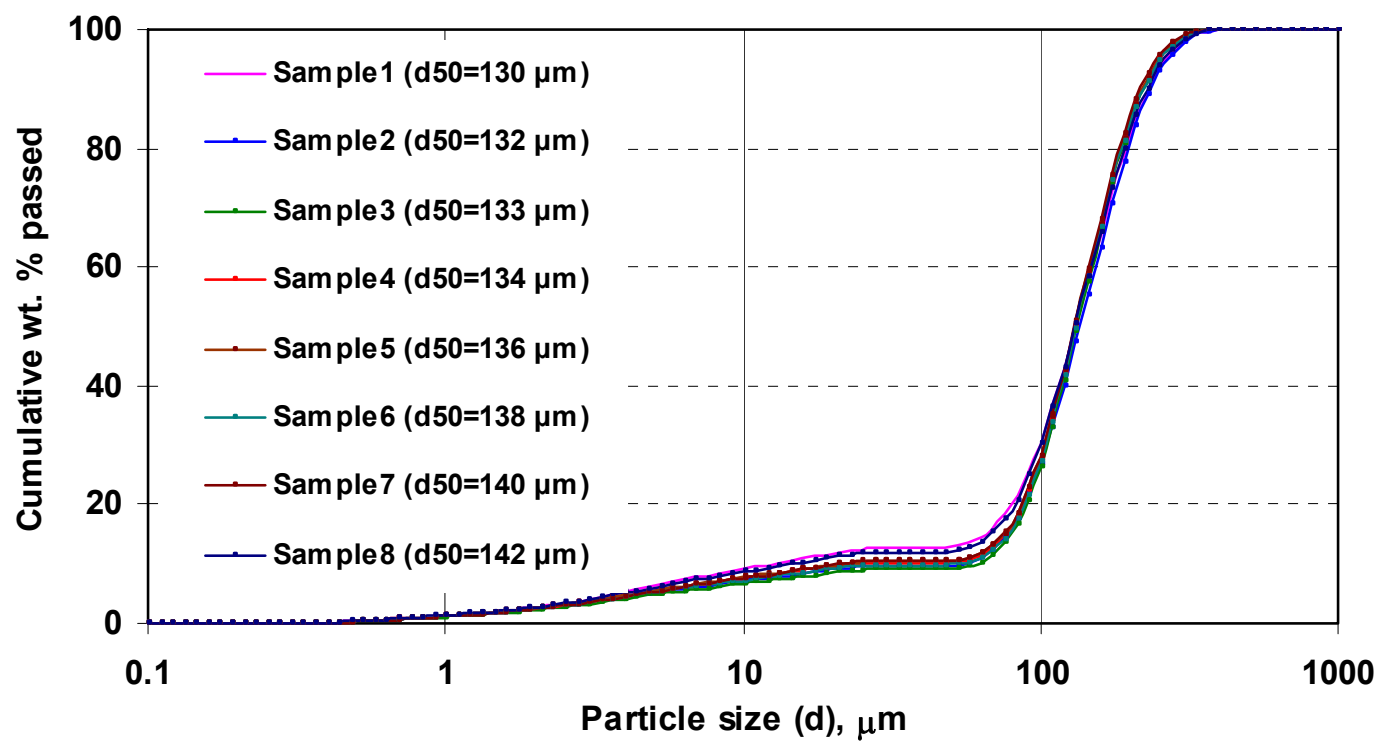

Fig. 6.5: Size distribution of eight samples produced by dividing a large volume sample.

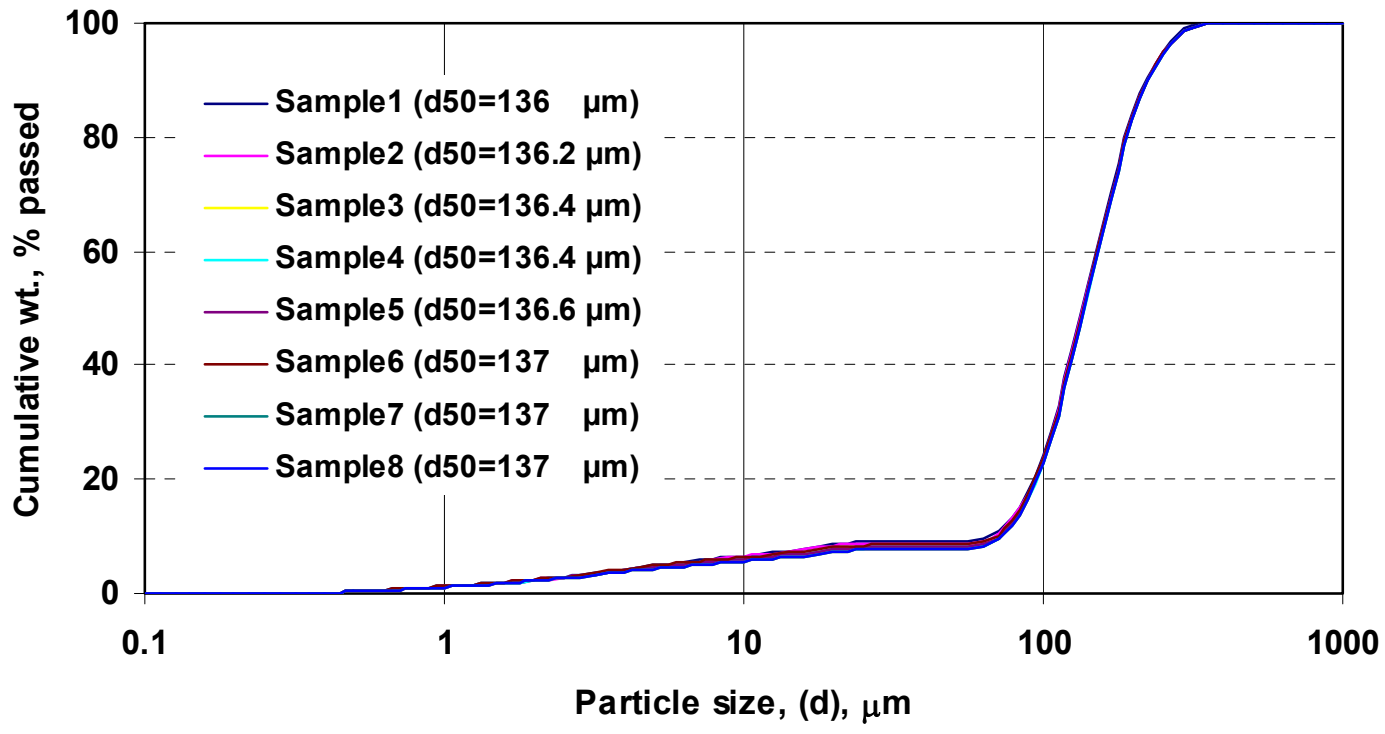

Fig. 6.6: Size distribution of eight samples produced by dividing a small volume sample.

In the present work, all the size analysis was made using the laser diffraction analyzer instrument which has a high accuracy compared with the other traditional analysis methods. So, the error percent which may arise from the size analysis is expected to be low. Most of these errors referred to the personal error of the operator and/or the preparation of the sample which will be analyzed. Another reason which should be taken into consideration is the particle size range of the sample. The closer the size 
ranges of the analyzed sample, the more accurate is the size analysis. A wide range of sizes of the sample may result in some deviations if the sample is to be repetitively analysed. So, to minimise the error percent, the operator of the instrument must have a good experience with the instrument before starting the analysis process. Also, the sample must be well prepared, homogeneous, and representative to the whole original sample.

\subsection{Statistical Error}

Statistical (random) error occurs because it is not possible to determine measurement conditions to infinite precision. This type of error is unpredictable and can not be eliminated. There are many sources of random error, such as equipment limitations, reading uncertainties and statistical fluctuations. Statistical (random) error can be evaluated through the statistical analysis. The relative error of any measured parameter using the student $t$ distribution can be given as follows [94,95]:

$$
(\delta U)=\frac{\sigma_{N^{:} \alpha / 2}}{\sqrt{N_{i}}},
$$

where,

$N_{i}$ is the number of independent observations, $t_{N^{\prime}: \alpha / 2}$ is the student $t$ variable with $N^{\prime}$ degrees of freedom, $N^{\prime}=N_{i}-1$, and $\sigma$ is the standard deviation which can be given as follows

$$
\left.\sigma=\sqrt{\left\{\frac{1}{N_{i}-1} \sum_{i=1}^{n}\left(x_{i}-\bar{x}\right)^{2}\right.}\right\},
$$

where, $x_{i}$ is the measured value and $\bar{x}$ is the mean value of all measurements. 


\subsubsection{Statistical Errors Affect the Separation Curve Determination}

The separation curve which characterizes the separation efficiency of the hydrocyclone is illustrated by treating the data obtained from the measured experimental values. These data are the mass flow rates and particle size distributions of both overflow and underflow products. Using these data, the separation curve can be constructed using Eq. (6.4).

Accordingly, it can be reported that the value of the separation efficiency of the fine particles on the separation curve $\left(T_{0}\right)$ which indicates the percentage of the finest size of the feed which miss reported into the underflow is a function of the mass flow rate and the weight fraction of each particle size $(d)$ for both overflow and underflow as follows:

$T_{0}=f(U, O, u(d), o(d))$.

So, the random error occurs in determining the separation curve can be calculated by computing the random error of these parameters measurements together.

\subsubsection{Statistical Error of Mass Recovery Determination}

In connection with the fact that the mass recovery in each product can be calculated using the measured values of the taken samples (weight, volume, and sampling time), many repetitions were carried out at the same conditions to estimate the statistical error occurred through mass fractions determination. The mass balance equation of the hydrocyclone can be given as follows;

$F=U+O$,

where, $F, U$, and $O$ are the solid flow rates of the feed, underflow, and overflow streams. According to Eq. (6.8) the relative error of overflow mass fraction $(\delta O)$ and of the underflow mass fraction $(\delta U)$ will be the same value. 
Table (6.1) and Table (6.2) show the experimental data of the mass balance which were obtained from five repetitions. These repetitions were carried out at the same operating conditions for underflow and overflow streams, respectively.

Table 6.1: Repetitions of underflow mass balance at $\left(D_{c}=50 \mathrm{~mm}, D_{0}=20 \mathrm{~mm}, D_{u}=4.2\right.$ $\mathrm{mm}, \mathrm{p}_{\mathrm{i}}=1$ bar, $\mathrm{IR}=3 \mathrm{l} / \mathrm{min}$ )

\begin{tabular}{|c|c|c|c|c|c|c|c|}
\hline $\begin{array}{c}\text { Repetition } \\
\text { No. }\end{array}$ & $\begin{array}{c}\text { Weight, } \\
\mathbf{k g}\end{array}$ & $\begin{array}{c}\text { Volume, } \\
\mathbf{l}\end{array}$ & $\begin{array}{c}\text { Density, } \\
\mathbf{k g} / \mathbf{m}^{\mathbf{3}}\end{array}$ & $\begin{array}{c}\text { Solid } \\
\text { content, } \\
\mathbf{k g} / \mathbf{l}\end{array}$ & $\begin{array}{c}\text { Time, } \\
\mathbf{s e c}\end{array}$ & $\begin{array}{c}\text { Solids } \\
\mathbf{f l o w} \\
\text { rate to } \\
\mathbf{U F}, \\
\mathbf{k g} / \mathbf{h}\end{array}$ & $\begin{array}{c}\text { Solid } \\
\text { recovery } \\
\text { to UF, \% }\end{array}$ \\
\hline 1 & 1.089 & 0.72 & 1513 & 0.823 & 18.56 & 115 & 15 \\
\hline 2 & 1.020 & 0.68 & 1500 & 0.803 & 18.75 & 105 & 16 \\
\hline 3 & 0.981 & 0.66 & 1486 & 0.781 & 18.78 & 100 & 16 \\
\hline 4 & 0.978 & 0.66 & 1482 & 0.774 & 19.34 & 95 & 15 \\
\hline 5 & 0.924 & 0.61 & 1515 & 0.827 & 18.38 & 99 & 16 \\
\hline
\end{tabular}

Table 6.2: Repetitions of overflow mass balance at $\left(D_{c}=50 \mathrm{~mm}, D_{0}=20 \mathrm{~mm}, D_{u}=4.2\right.$ $\mathrm{mm}, \mathrm{p}_{\mathrm{i}}=1$ bar, $\mathrm{IR}=3 \mathrm{l} / \mathrm{min}$ )

\begin{tabular}{|c|c|c|c|c|c|c|}
\hline $\begin{array}{c}\text { Repetition } \\
\text { No. }\end{array}$ & $\begin{array}{c}\text { Weight, } \\
\mathbf{k g}\end{array}$ & Volume, I & $\begin{array}{c}\text { Density, } \\
\mathbf{k g} / \mathbf{m}^{\mathbf{3}}\end{array}$ & $\begin{array}{c}\text { Solid } \\
\text { content, } \\
\mathbf{k g} / \mathbf{l}\end{array}$ & $\begin{array}{c}\text { Solids flow } \\
\text { rate to } \\
\mathbf{O F}, \mathbf{k g} / \mathbf{h}\end{array}$ & $\begin{array}{c}\text { Solid } \\
\text { recovery } \\
\text { to OF, \% }\end{array}$ \\
\hline 1 & 1.946 & 1.780 & 1093 & 0.149 & 4450 & 85 \\
\hline 2 & 1.924 & 1.780 & 1080 & 0.129 & 4161 & 84 \\
\hline 3 & 1.902 & 1.730 & 1068 & 0.159 & 4500 & 84 \\
\hline 4 & 2.803 & 2.600 & 1078 & 0.125 & 4100 & 85 \\
\hline 5 & 1.689 & 1.580 & 1069 & 0.110 & 3980 & 84 \\
\hline
\end{tabular}


From Table 6.1 and Table 6.2, the statistical error in the mass recovery was determined by using Eq. (6.5) and Eq. (6.6) at 95\% confidence interval, the standard error $\sigma$ and the relative error $(\delta U)$ of the underflow mass fraction were found to be about $0.6 \%$ and $0.4 \%$, respectively. So, the standard error and the relative error $(\delta O)$ of the overflow mass fraction are also the same values, respectively.

\subsubsection{Statistical Error of Size Analysis}

According to formula (6.7), the particle size distribution of both overflow and underflow streams represents the second variable which is required to illustrate the partition curve. The classification point can be chosen anywhere along this curve. The choice of these points depends on the undesirable fraction to be removed or the recovery of the desired value [8]. In the present work, the fines (kaolin) in the underflow stream were represented by the percentage of the size of minus $10 \mu \mathrm{m}$ in the underflow product. This percentage should be minimised as much as possible with water injection. Concerning the overflow stream, the size plus $61 \mu \mathrm{m}$ was chosen to characterise the overflow quality. The overflow which is the final product should not contain more than $2 \%$ greater than this size.

In the present work, five separate test series (i.e., after each test, the pump is turned off and after that turned on again to make the second test and so on.) were carried out at the same operating conditions. At every test an overflow and an underflow samples were taken and analysed. This procedure was made to include the error arises during achieving the steady state conditions at every test. In addition to this procedure, onetest samples (overflow and underflow) were repetitively analysed 5 times also for each sample to involve the error of the analysing process itself (preparation of the representative sample, way of introducing the sample to the analyzer, laser intensity, and volume of the sample injected to the analyzer).

Substituting the measured values of the percentage of sizes $10 \mu \mathrm{m}$ and $61 \mu \mathrm{m}$ in overflow and underflow samples respectively into Eq. (6.5) and Eq. (6.6) at 95 percent confidence interval, the relative error of measuring the size $10 \mu \mathrm{m}$ in the 
underflow was found to be $0.1 \%$ and the relative error of measuring the size $61 \mu \mathrm{m}$ in the overflow was found to be $0.2 \%$. This low error percent may be due to the high accuracy of the Mastersizer instrument, the proper preparation of the representative sample, and finally the experience of the operator which was gained through high training and many repetitions of the analysis using this instrument.

\subsection{Experimental Error Evaluation}

Figure 6.7 shows a schematic representation of the values of the different expected sources of error which may occur during the experimental work as discussed above.

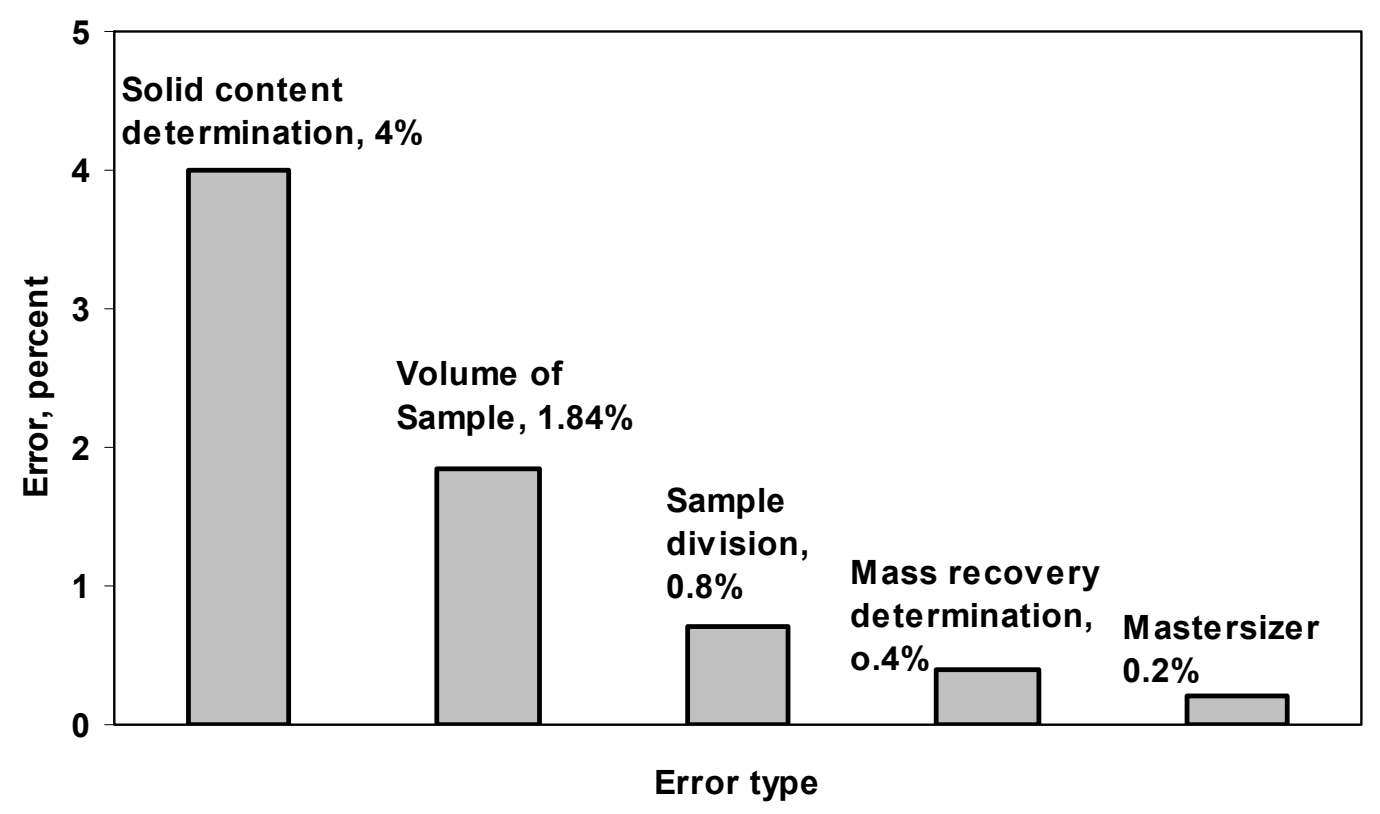

Fig. 6.7: Values of the different error sources occur through the experimental work.

As can be seen from Fig. 6.7, the highest error percent which is about 4 percent is within the accepted range of error. Fig. 6.7 shows also that the error arose from the solid content determination using the formula is higher than the other error values. However, this error percentage can be considered small (4\%). This may be due to the relative error of the suspension density determination. This indicates that this process should be accurately carried out through the experiments even in the case of using high feed solid content. Therefore, several repetitions should be made before starting the sampling process to minimise the percentage of this error as much as possible. On 
the other side, the error percent arose from the Mastersizer analysis is very low $(0.2 \%)$. This may be due to the high accuracy of this instrument and the low personal effect through the analysis process.

\subsection{Reproducibility of the Separation Curve Determination}

To investigate the effect of the over all percentage error arises from the above discussed sources together on the separation curve, five test repetitions were carried out at the same operating conditions. The percentage error was calculated for the different sizes used to construct the separation curve and the results are shown in Fig 6.8. The separation curve was drawn using a log-log scale to show the relative error clearly.

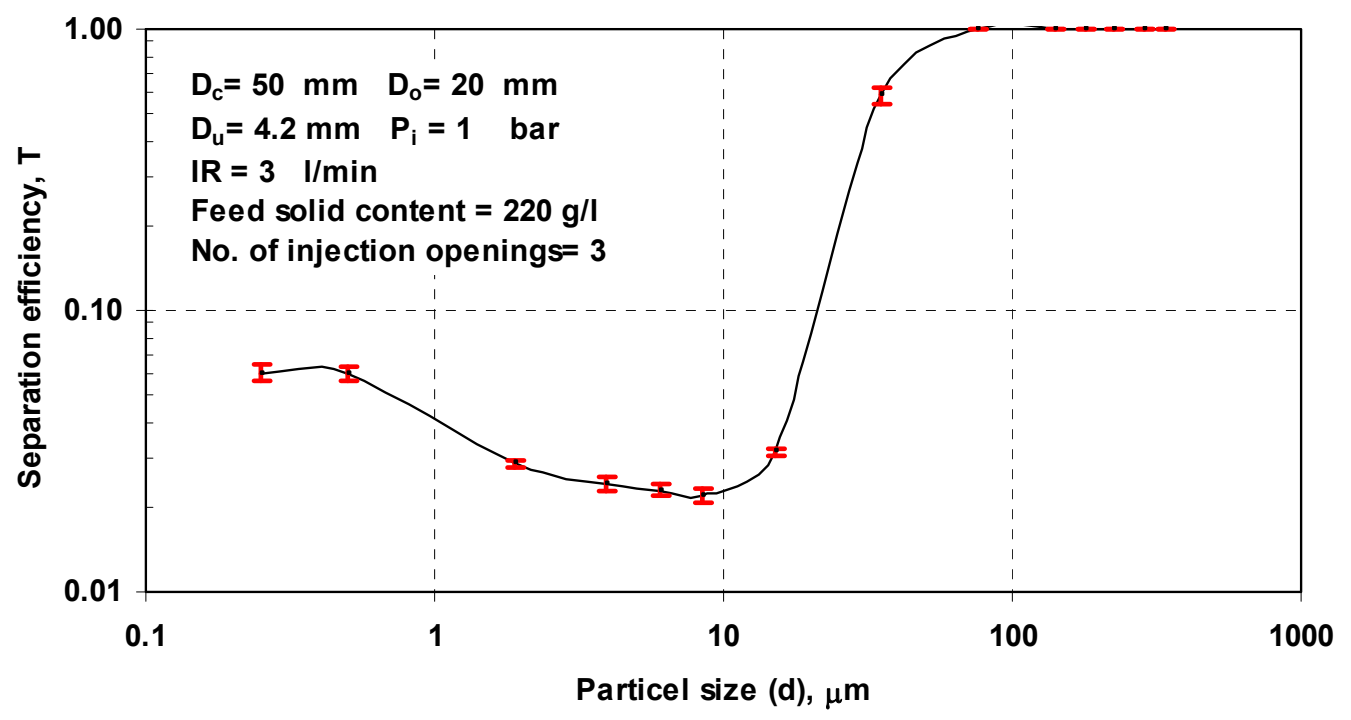

Fig. 6.8: Separation curve determined from five test repetitions.

The results of the reproducibility test reported that the experiments are reproducible with a maximum error of 7 percent with a 95 percent confidence interval which can be considered within the acceptable range of error $(10 \%)[94,95]$. The low percentage error may be due to the proper procedure followed in the preparation of the representative samples of the suspension and the high accuracy of the particle size analyzer (Mastersizer $X$-2000) which is used in the analysis. However, these percentage errors should be taken into consideration through carrying out the experimental work. 


\section{Results and Discussion}

\subsection{Investigation of the $\mathbf{5 0} \mathbf{~ m m}$ Cyclone (Without Water Injection), Using Water Only}

The experiments were carried out using the $50 \mathrm{~mm}$ cyclone with only water as feed and without injection to study the effect of feed pressure on the water flow rate through the feed, overflow, and underflow openings. The tangential velocity at the hydrocyclone inlet was also determined at different feed pressures.

Figure 7.1 shows the effect of the feed pressure on the water flow rate $(1 / \mathrm{min})$ of the feed, overflow, and underflow streams. It shows also, the equivalent tangential velocity $V_{\text {tang }}$ at the hydrocyclone inlet of each feed pressure.

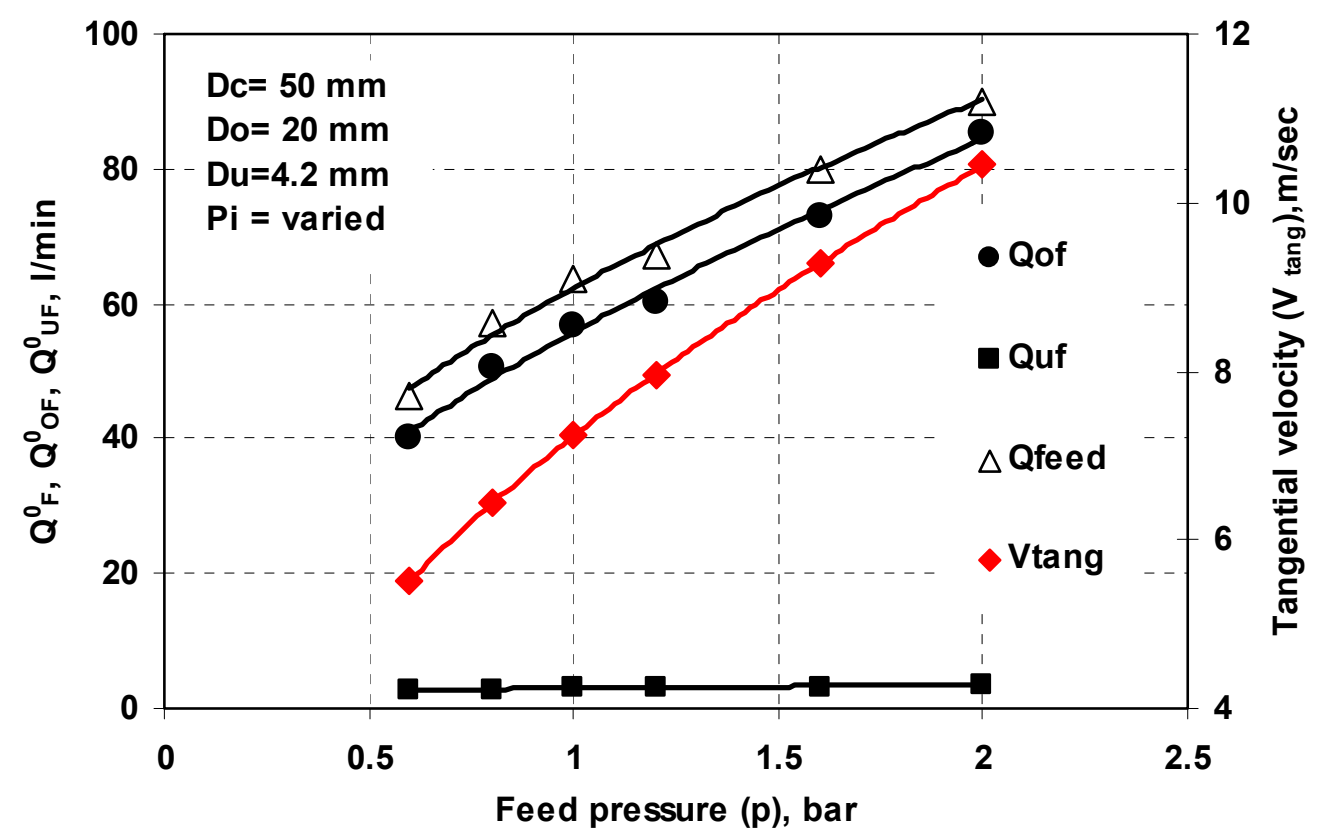

Fig. 7.1: Effect of feed pressure on the feed tangential velocity and the flow rates of feed, overflow, and underflow.

From Fig. 7.1, it can be shown that by increasing the feed pressure $p$ (bar), the feed flow rate $Q_{F}^{0}(1 / \mathrm{min})$ and overflow rate $Q_{O F}^{0}(1 / \mathrm{min})$ are increasing gradually while the underflow flow rate $Q_{U F}^{0}(1 / \mathrm{min})$ has a slight increase. From these results, the 
following empirical equations with correlation coefficients rang between 0.97 and 0.99 can be derived:

$Q_{F}^{0}=62.37 \cdot p^{0.53}$,

$Q_{O F}^{0}=55.79 \cdot p^{0.59}$

$Q_{U F}^{0}=3 \cdot p^{0.21}$

The tangential velocity $V_{\text {tang }}(\mathrm{m} / \mathrm{sec})$ was calculated using the following Eq. (7.4)

$V_{\tan g}=\frac{Q_{F}^{0}}{A_{i}}$

where, $Q_{F}^{o}$ can be determined from Eq. (7.1) for each pressure value $p$ (bar) and $A_{i}$ is the inlet area of the hydrocyclone $\left(\mathrm{mm}^{2}\right)$.

From Fig. 7.1, Eq. (7.1), Eq. (7.2), and Eq. (7.3), it can be concluded that the higher feed pressure result in an increase of both outlets flows (overflow and underflow) in different proportions. It can also be seen that the tangential velocity increases by increasing the feed pressure. This may be due to the increase in the hydrocyclone throughput and the centrifugal force by increasing the feed pressure. These empirical equations were found in agreement with the reviewed empirical equations of Tervonsky [96].

The volume split parameter (S) plays an important role in the hydrocyclone separation. The split parameter $(\mathrm{S})$ can be defined as the ratio of the overflow water flow rate $\left(Q_{O F}^{0}\right)$ to the underflow water flow rate $\left(Q_{U F}^{0}\right)$. The dependency of the split parameter was investigated by many researchers [97-100]. They assumed that the volume split parameter is simply related to the diameters of the outlet openings (overflow and underflow openings) as follows; 
$S=\frac{D_{o}}{D_{u}}$

It was found that the split parameter depends also on the feed pressure. In the present work, this dependency was investigated and the following empirical equation was obtained:

$S=x \cdot p^{a}$,

where, $x=20.9$ and $a=0.56$. Figure 7.2 shows the effect of the feed pressure on the split parameter. The dependency of the split parameter on the feed pressure was found in the literature to have the same relationship with different values of the constant $x$ and the exponent $a$. These values were in the range between 1 and 43.77 for the constant $x$ and between 0 and 1.3 for the exponent $a$. From Eq. (7.6), it can be reported that the experimental results showed a moderate dependency of the split parameter $(\mathrm{S})$ on the feed pressure $(\mathrm{p})$. This relation agrees with the results of Shah et al. [100]

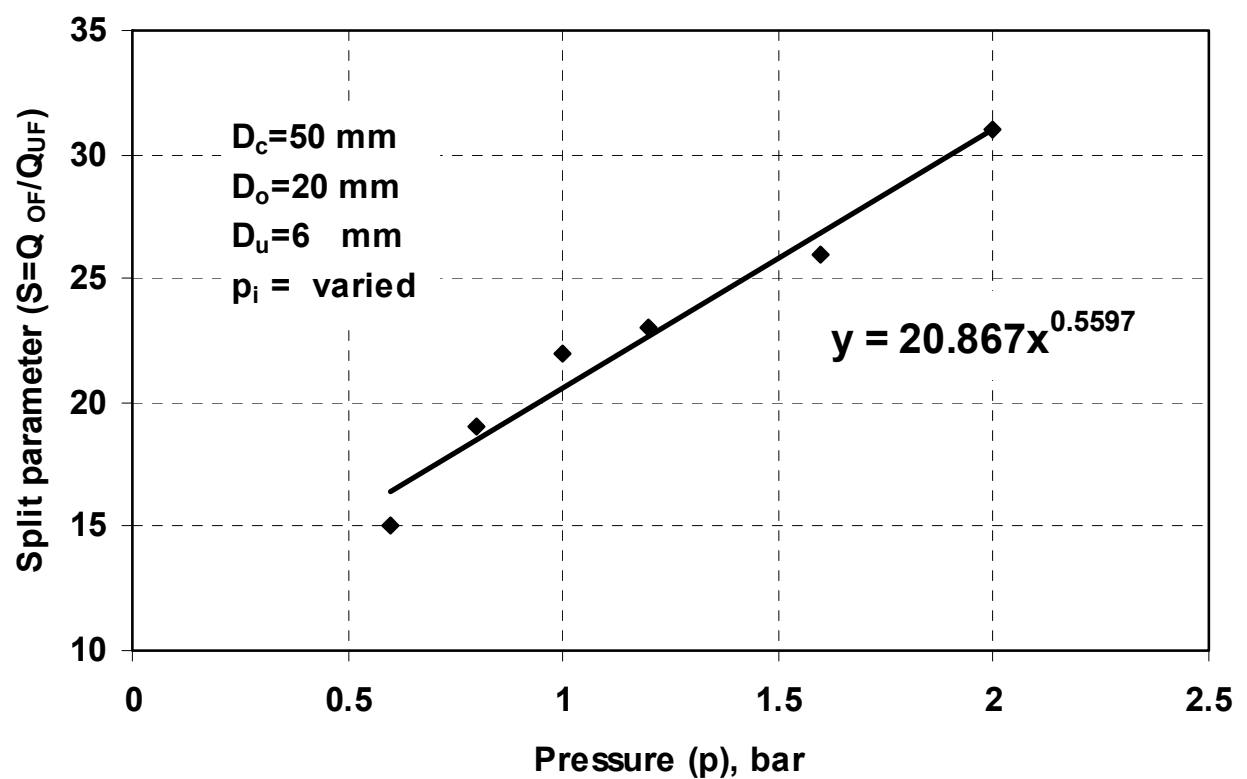

Fig. 7.2: Effect of feed pressure on the volume split parameter $\left(S=Q_{O F}^{0} / Q_{U F}^{O}\right)$ without water injection. 
So, it can be concluded that the split volume parameter $(S)$ does not have a unique dependency on the feed pressure (p) and has to be determined for every individual hydrocyclone's geometry.

\subsection{Investigation of the $50 \mathrm{~mm}$ Cyclone (with Water Injection), Using Water Only}

The experiments were carried out at different water injection rates $(1 / \mathrm{min})$, different feed pressures (bar), and different numbers of injection openings.

\subsubsection{Effect of Injection Rate on the Overflow and Underflow Rates}

The fine particles are separated proportional to the ratio of water flow rates through the overflow and underflow openings. Therefore, it was important to study the effect of the water injection on the water distribution through underflow and overflow openings.

Figure 7.3 shows the effect of the injection rate (IR) in $1 / \mathrm{min}$ on the ratio of $\Delta Q_{O F} / Q_{O F}^{0}$. Here, $\Delta Q_{O F}$ is the difference between the overflow water rate with

injection $Q_{O F}^{i n}$ and the overflow water rate without injection $Q_{O F}^{0}$ at the same experimental conditions. These sets of experiments were carried out using 3 injection openings. 


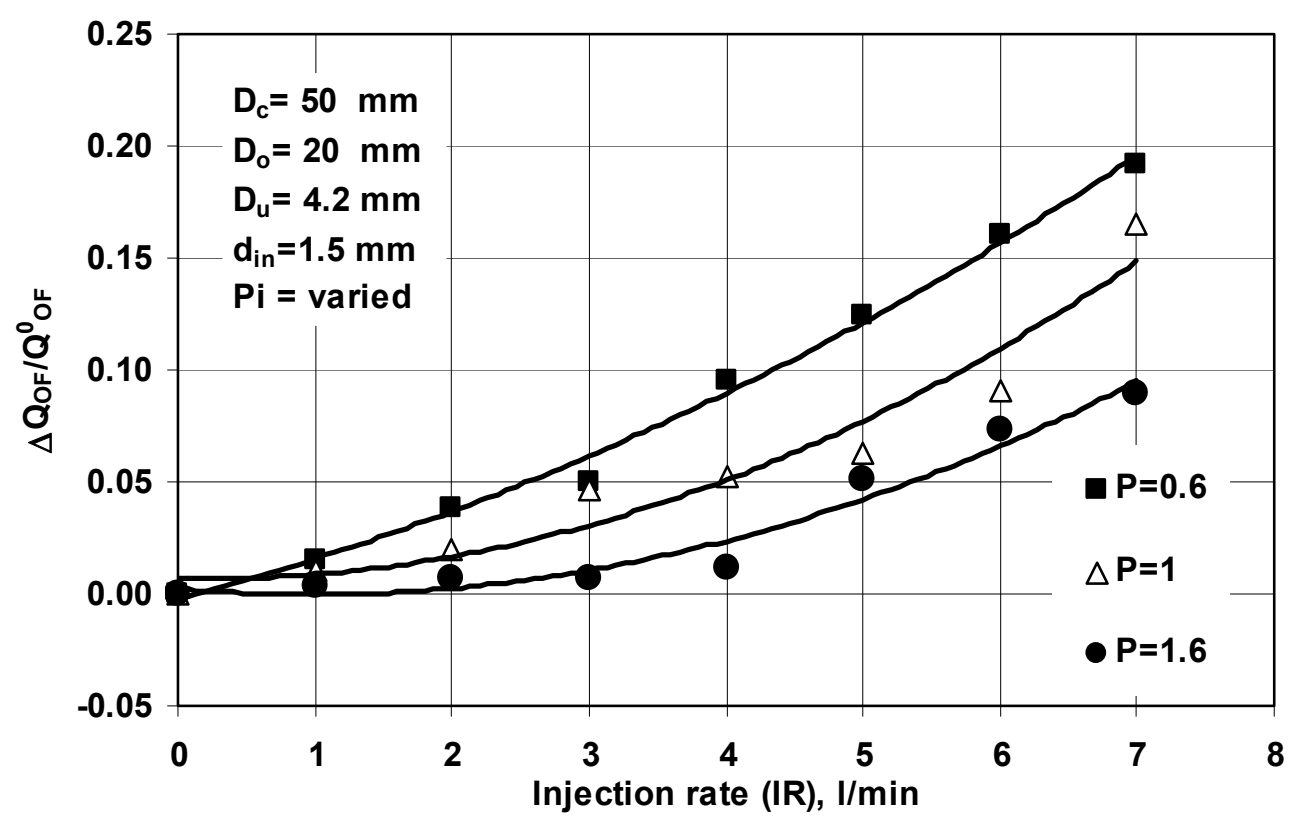

Fig. 7.3: Effect of water injection rate on the overflow rate $\left(\Delta Q_{O F} / Q_{O F}^{0}\right)$.

From Fig. 7.3, it can be shown that the $\Delta Q_{O F}$ increases gradually with increasing the injection rate. This shows that the injected water going to the overflow stream increases with increasing the injection rate which is the desired effect to carry the fines to the overflow. Figure 7.3 shows also that the increase in the split parameter in the case of high pressure ( 1.6 bar) is lower than its increase in the case of low pressure ( 0.6 bar). This may be due to the fact that at high feed pressure, the centrifugal force increases which result in high back pressure at the cyclone wall. This may explain the observation about the low injection effect at high pressure values.

Figure 7.4 shows the effect of the injection rate (IR) in $1 / \mathrm{min}$ on the ratio of $\Delta Q_{U F} / Q_{U F}^{0}$. Here, $\Delta Q_{U F}$ is the difference between the underflow water rate with injection $Q_{U F}^{i n}$ and the underflow water rate without injection $Q_{U F}^{0}$ at the same experimental conditions. 


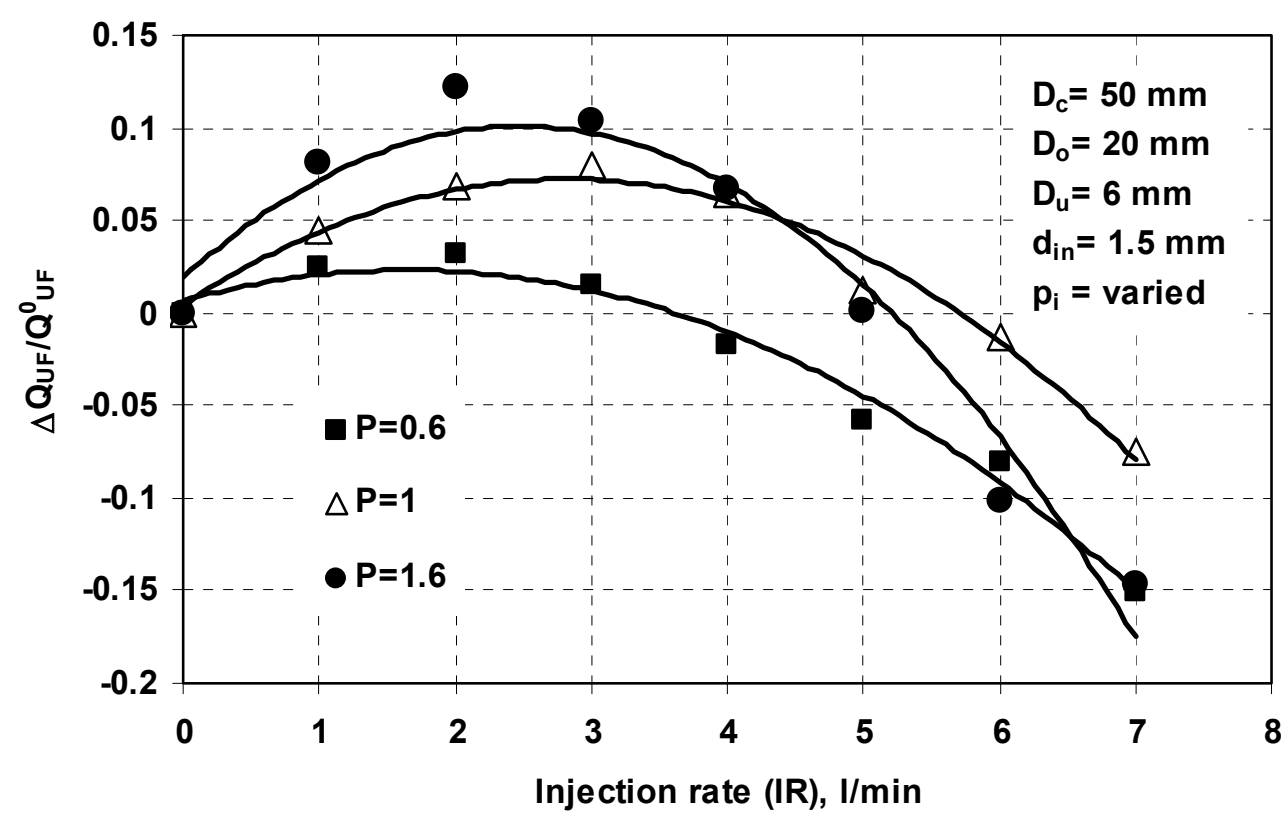

Fig. 7.4: Effect of injection rate on the underflow rate $\left(\Delta Q_{U F} / Q_{U F}^{0}\right)$.

From Fig.7.4, it can be seen that the dependence of flow rate through the underflow is more complicated. The experiments show that at low injection rates, the underflow throughput increases while it decreases with further increase in the injection rates. This can be explained as follows:

At low injection rate, the injection velocity is relatively small and can not reach the inner vortex of the hydrocyclone. Accordingly, it will be entrained by the entire vortex and discharges through the underflow. At high injection rates, the underflow water rate decreases. This can be explained as follows:

By increasing the injection rate, the injection velocity increases and becomes higher than the tangential velocity of the cyclone flow. This will intensify the main flow inside the hydrocyclone and then the air core diameter will increase causing a suction effect upward. Accordingly, little or no water will go to the underflow discharge, which is clear at high pressure.

\subsubsection{Effect of Injection Rate on the Volume Split Parameter (S) Using 3 Injection Openings}


Figure 7.5 shows the effect of the injection rate on the volume split parameter (S) using three injection openings at different feed pressures.

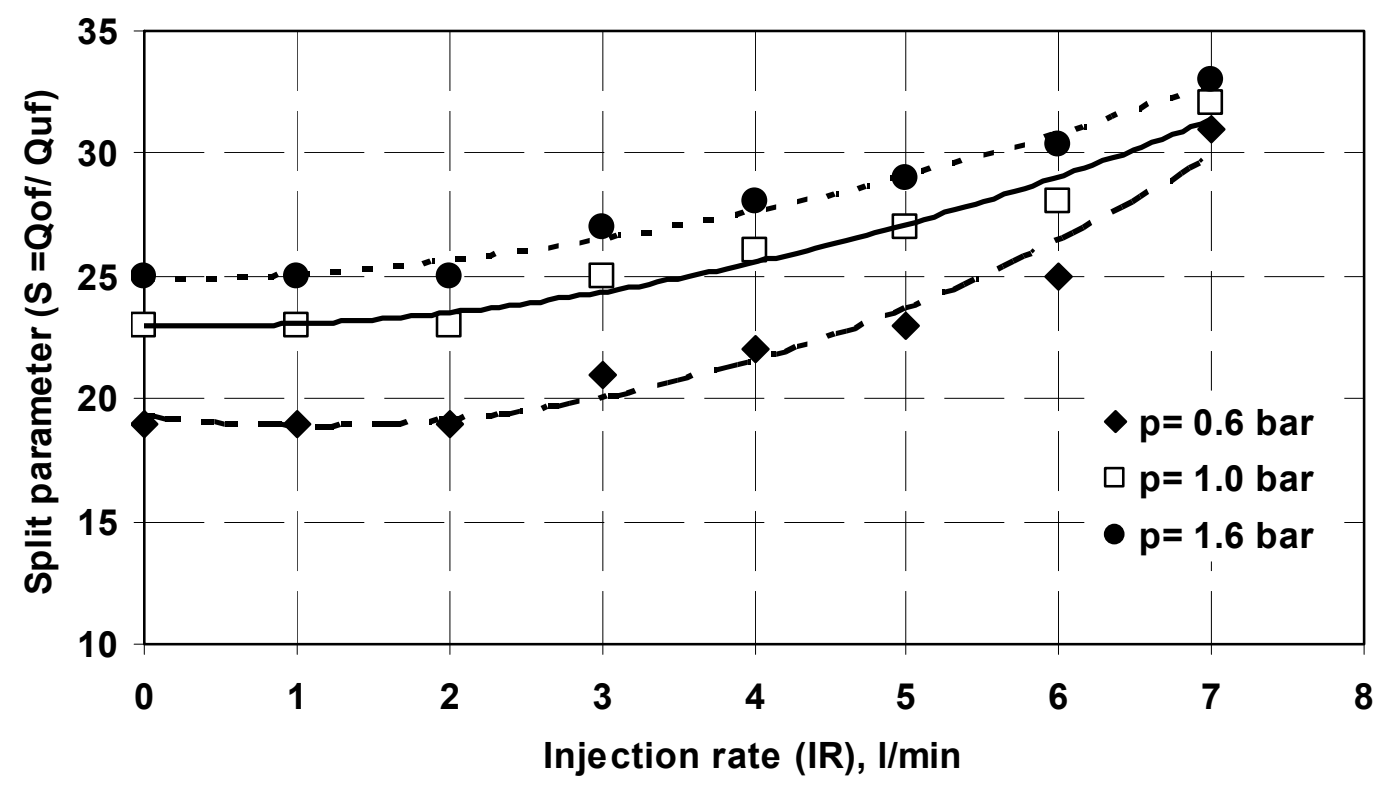

Fig. 7.5: Effect of injection rate on the volume split parameter (S) at different feed pressures.

Figure 7.5 shows that the change in the split parameter is almost remains stable which means that both overflow and underflow rates are increased. At high injection rates, the split parameter increases with increasing the injection rate. This can be explained by the increase in the overflow rate and the decrease in the underflow rate which occur at high injection rates as it is shown in Fig. 7.3 and Fig. 7.4.

\subsubsection{Effect of Injection Rate on the Volume Split Parameter (S) Using 5 Injection Openings}

The effect of the injection rate on the volume split parameter using five injection openings was investigated. The experiments were supported by model simulation using Fluent software [10]. The hydrocyclone parameters used in these experiments and in the simulations are as follows:

The hydrocyclone diameter $D_{c}=50 \mathrm{~mm}$, hydrocyclone length $L=600 \mathrm{~mm}$, inlet opening diameter $D_{i}=14.5 \mathrm{~mm}$, underflow diameter $=8 \mathrm{~mm}$, overflow diameter $D_{o}=$ $16 \mathrm{~mm}$, Area of the inlet opening $A_{i}=165 \mathrm{~mm}^{2}$, number of injection openings $N=5$. Figure 7.6 and Fig. 7.7 show a comparison between the experimental and calculated 
results of the split parameters versus the injection rate at feed pressure of 0.5 bar and 1.5 bar respectively.

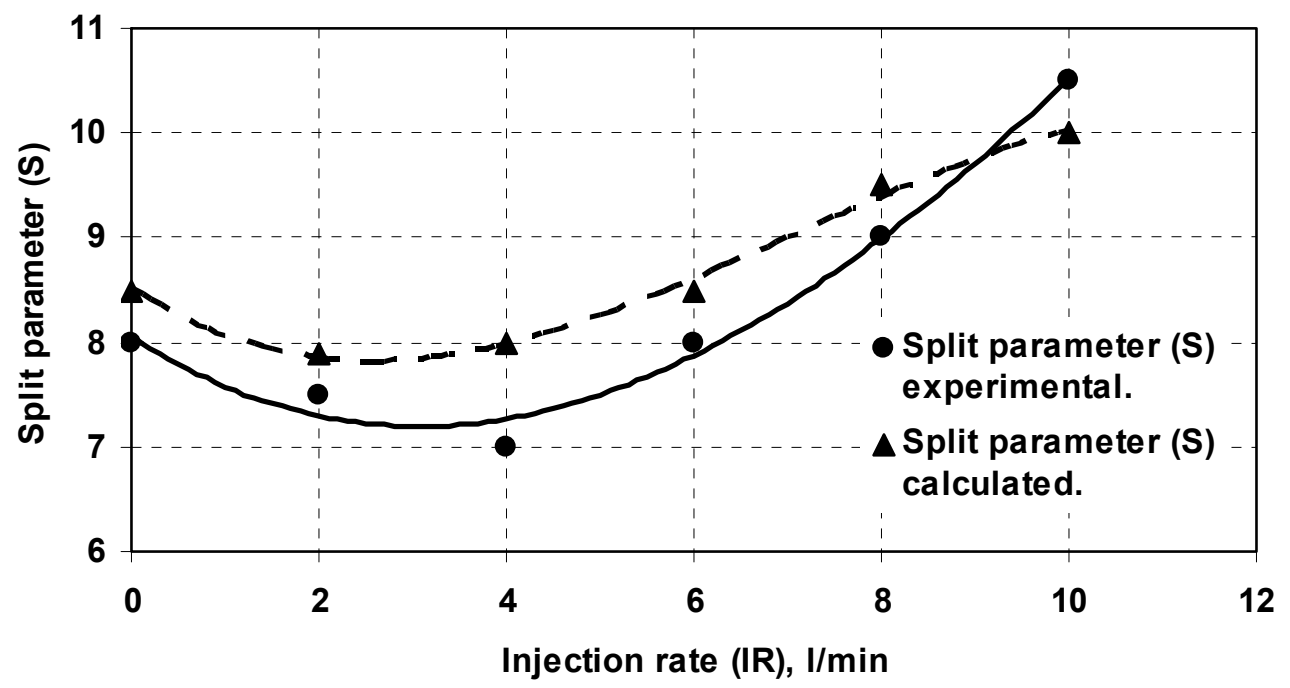

Fig. 7.6: Comparison between experimental and calculated values of the split parameter $(\mathrm{S})$ as a function of the water injection rate at feed pressure $=0.5$ bar.

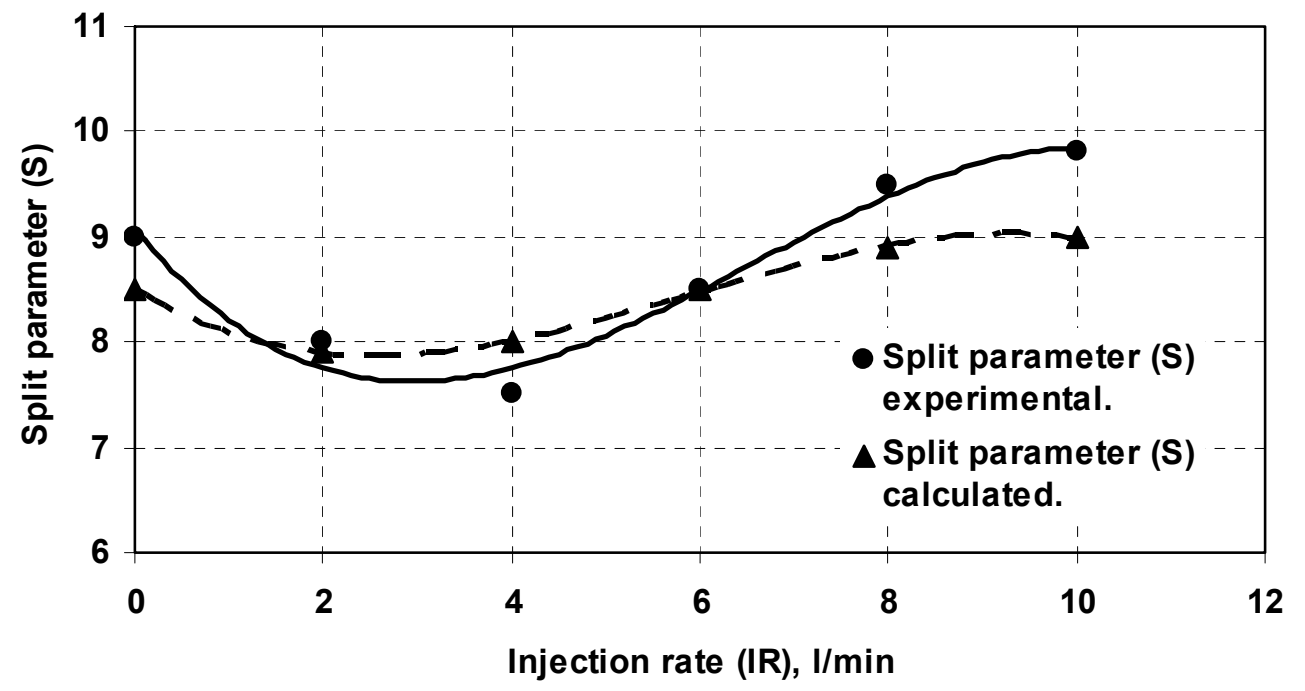

Fig. 7.7: Comparison between experimental and calculated values of split parameter (S) as a function of the water injection rate at feed pressure $=1.5$ bar.

From Figures 7.6 and 7.7 it can be seen that, at low injection rates, the split parameter decreases at the beginning of the injection and then starts to increase at further increase in the injection rate. This is due to the difference between the injection 
velocity values at low and high injection rates which was previously discussed in the discussion of Fig. 7.4. Figure 7.6 and Fig. 7.7 show also that both the experimental and the calculated results are within reasonably agreement. So, it is indicated that the influence of the injection rate on the split parameter depends on the injection velocity. The effect of the injection velocity will be discussed in detail in the next chapters.

\subsection{Classification in the $50 \mathrm{~mm}$ Hydrocyclone With Water Injection}

During the present work, the dependency of the percentage of the fine in the underflow product on the different design and operating parameters of the $50 \mathrm{~mm}$ water injection hydrocyclone were examined. The parameters were systematically investigated as follows:

- Injection geometry,

- Injection rate,

- Hydrocyclone geometry,

- Feed pressure,

- Feed conditions.

The methodology of one variable at a time was used during the experiments where only one parameter is changed and the other parameters are kept constant. The evaluation of the results was based mainly on the interpretation of the separation curve and the developed theoretical model. The effect of the derived parameters on the characteristics of the separation process such as the cut size $\left(d_{50}\right)$, the underflow solid recovery, and the underflow solid content were evaluated. A further concern associated with the water injection cyclone is the effect of the injection on the classification sharpness characterised by the imperfection value (I) which is determined from the actual separation curve using Eq. (1.2). Accordingly, an imperfection value of zero represents the ideal (theoretical) classification sharpness.

\subsubsection{Effect of Injection Direction}

The water injection direction is considered an important parameter in determining the manner in which the water will follow inside the hydrocyclone, because the injected 
water will form a continuous flow with a definite direction inside the hydrocyclone. To investigate the effect of the injection direction, three different injection rings with different directions were designed. These designed directions were:

- Tangential direction in the same direction as the main flow through the hydrocyclone

- Tangential direction against the direction of the main flow inside the hydrocyclone

- Radial direction (perpendicular to the hydrocyclone axis).

\subsubsection{Effect of Injection Direction on the Separation Efficiency of the Fine Particles}

$$
\left(\mathbf{T}_{0}, \mathbf{T}_{\min }\right)
$$

Figure 7.8 shows different separation curves obtained at different injection directions. Figure 7.9 shows the effect of changing the injection direction on the separation efficiency values of the fine particles $\left(\mathrm{T}_{0}, \mathrm{~T}_{\mathrm{min}}\right)$. The $T_{0}$ value is considered the fine particle entrainment by coarse settled particles (the fish hook) while the $T_{\min }$ value is the minimum point on the separation curve which represents the fines related to the water recovery to the underflow.

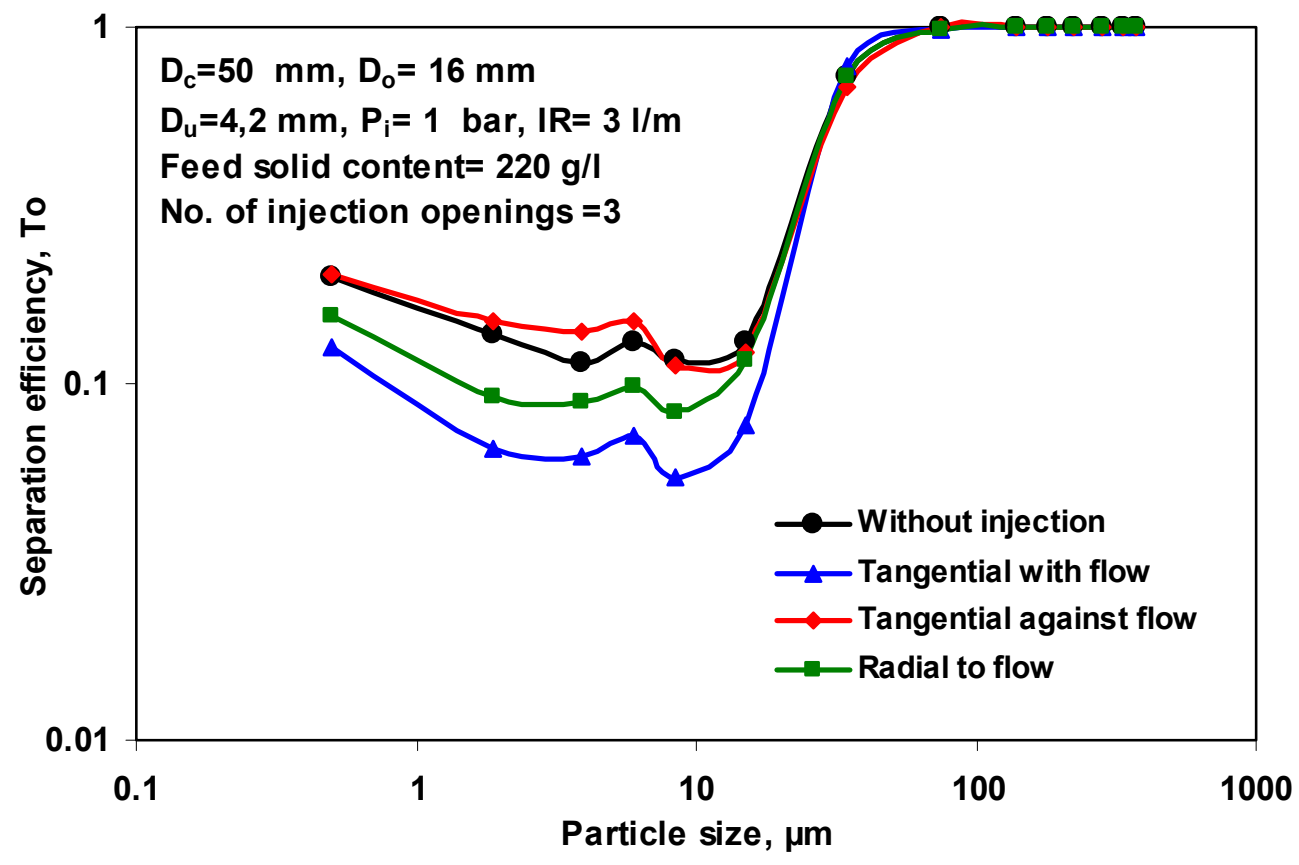

Fig. 7.8: Different separation curves obtained at different injection directions. 


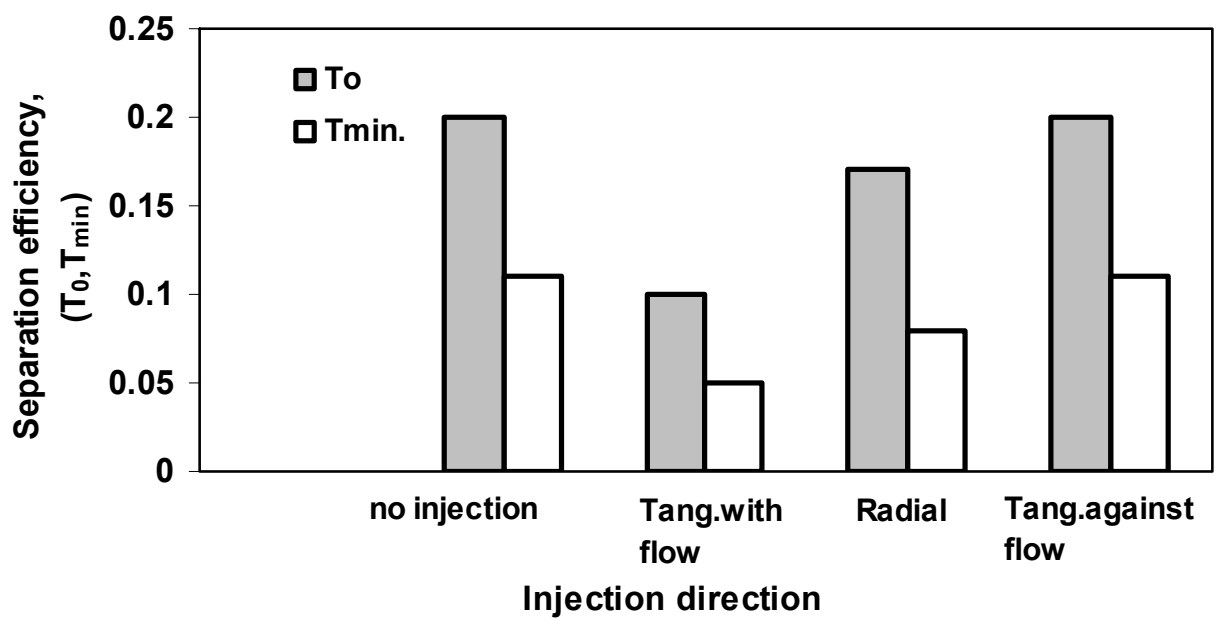

Fig. 7.9: Effect of injection direction on the separation efficiency of the fine particles $\left(\mathrm{T}_{0}, \mathrm{~T}_{\min }\right)$.

As can be seen from Fig. 7.9, the $T_{\min }$ values run parallel to the $T_{0}$ values. It should be noted that in the treatment of all experiments, it was realized that both $T_{0}$ and $T_{\min }$ have always the same trend. Therefore, the effect of the investigated parameters on $T_{0}$ only, will be discussed.

Figure 7.8 and Figure 7.9 show that the tangential direction within the flow has the best effect because the lowest $T_{0}$ value was achieved. The radial injection showed also some improvement effect but lower than the effect of the tangential direction. On the other hand, the tangential direction against the flow has almost no effect, where the $T_{0}$ was the same as without injection.

In an attempt to explain the effect of the injection direction on the separation curve, Table (7.1) shows the expected potential changes in the main parameters which govern the suspension flow inside the hydrocyclone. The degree of the effect was expressed by the arrows direction and their length. The upward arrow indicates an increase and the downward arrow indicates a decrease. Further, the length of the arrow shows the degree of the change (low effect, medium effect, and high effect for short, medium, and long arrow length, respectively. 
Table 7.1: Qualitative evaluation of the injection direction effects on the tangential velocity, radial component of injection velocity, and the turbulent diffusion coefficient.

\begin{tabular}{|c|c|c|c|}
\hline \multirow[t]{2}{*}{ Parameter } & \multicolumn{3}{|c|}{ Injection direction } \\
\hline & $\begin{array}{l}\text { Tangential } \\
\text { with flow }\end{array}$ & Radial & $\begin{array}{c}\text { Tangential } \\
\text { against flow }\end{array}$ \\
\hline 1- Tangential velocity, $V_{\text {ta }}$ & & & \\
\hline $\begin{array}{c}\text { 2- Turbulent diffusion } \\
\text { Coefficient, } \mathrm{D}_{\mathrm{t}}\end{array}$ & & & \\
\hline $\begin{array}{l}\text { 3- Radial component of the } \\
\text { injection } \\
\quad \text { velocity, } V_{\text {in,r. }}\end{array}$ & ( & & \\
\hline
\end{tabular}

From Table (7.1) and Eq. (4.16) which expresses the $T_{0}$ value in the case of low injection rates (up to $31 / \mathrm{min}$ ), it can be clearly seen that the tangential injection with the flow has a lower $\mathrm{T}_{0}$ value due to the higher radial component of the injection velocity $\left(V_{r}\right)$ and the lower turbulent diffusion $\left(D_{t}\right)$. Also, the radial direction has a relatively moderate effect if compared with the case without injection due to the relatively high $V_{r}$ and low $D_{t}$ values. In the case of tangential direction against the flow, the high $T_{0}$ value can be easily explained due to the low $\left(V_{r}\right)$ and the high $D_{t}$ values.

\subsubsection{Effect of Injection Direction on the Cut Size $\left(d_{50}\right)$ and Imperfection (I)}

Figure 7.10 and Fig. 7.11 show the effect of the injection direction on the cut size $\left(\mathrm{d}_{50}\right)$ and the imperfection (I) respectively. 


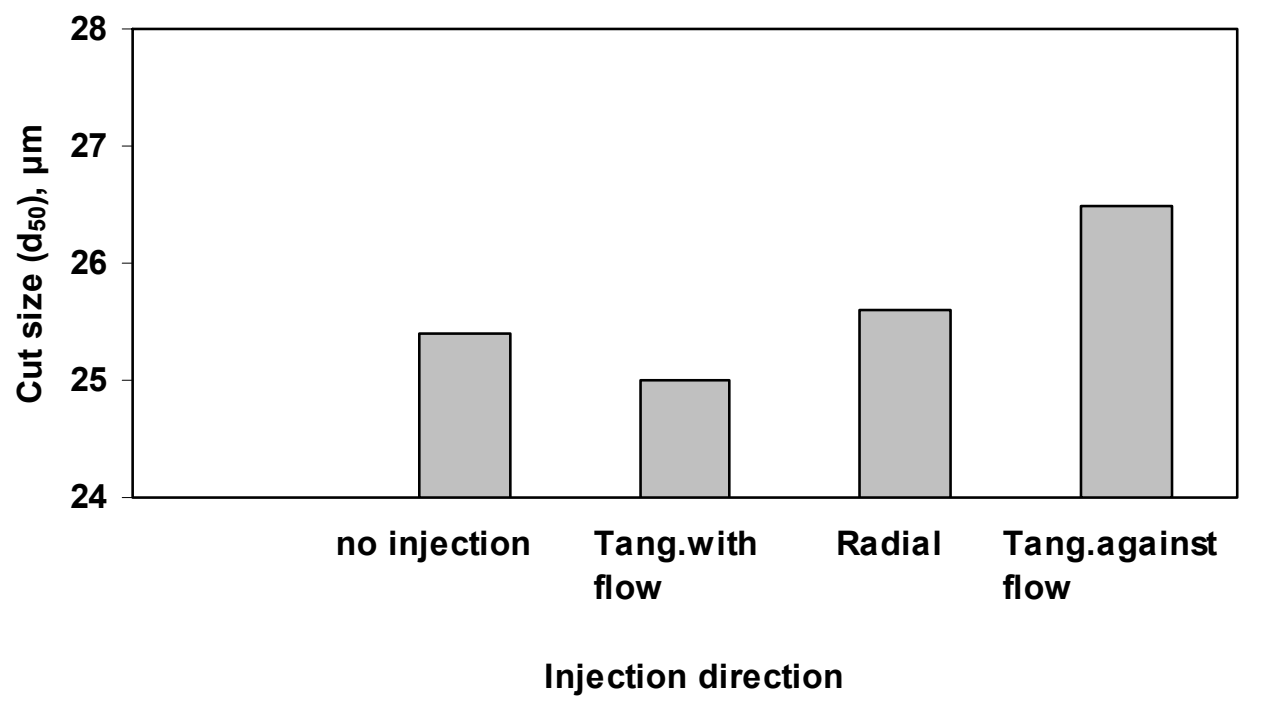

Fig. 7.10: Effect of injection direction on the cut size $\left(\mathrm{d}_{50}\right)$.

From Fig 7.10, it can be shown that the cut size $\left(\mathrm{d}_{50}\right)$ decreases slightly in the case of the tangential direction with the flow and increases in the radial direction and more increased in the case of tangential direction against the flow. This can be explained considering Eq. (4.15) from which $\mathrm{d}_{50}$ is inversely proportional to the settling velocity $\left(\mathrm{V}_{\mathrm{s}}\right)$ which is a function of the tangential velocity $\left(\mathrm{V}_{\text {tang }}\right)$. From Table (7.1), it can be shown that the tangential velocity increases in the case of tangential direction with the flow and reversed in the radial direction and reversed more in tangential direction against flow.

Figure 7.11 shows that the separation sharpness was improved by injecting the water in a tangential direction with the main suspension flow direction in the hydrocyclone. This may be due to the low turbulent diffusion occurs in the case of the tangential direction with the flow as can be seen from Eq. (4.15) and Table (7.1). Also the dilution effect which is caused by the tangential injection leads to better sharpness as can be seen from Eq. (4.18). The increase in the imperfection values in the radial and tangential against flow compared with the tangential direction may be due to the increase in the turbulence produced by the shear stresses which increases the turbulent diffusion causing imperfection values to be increased. It is not expected that the tangential water injection against the flow to have a better imperfection value than without injection. This may be due to the high dilution of the underflow which is 
according to Eq. (4.15) increases the settling velocity which improves the separation compared with no injection case. Also, the high turbulent diffusion in this case causes the captured fines to be released from the coarse sediment.

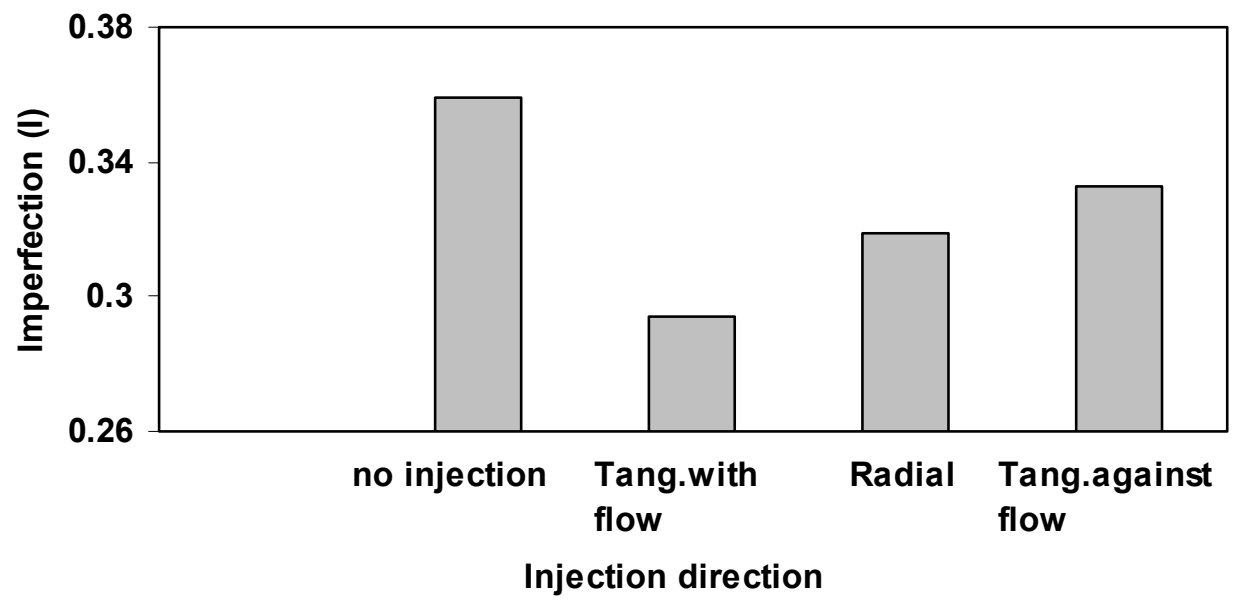

Fig. 7.11: Effect of injection direction on the imperfection.

\subsubsection{Effect of Injection Direction on the Solid Content and Solid Recovery}

Figure 7.12 and Fig. 7.13 show the effect of the injection direction on the solid content and the solid recovery of the underflow.

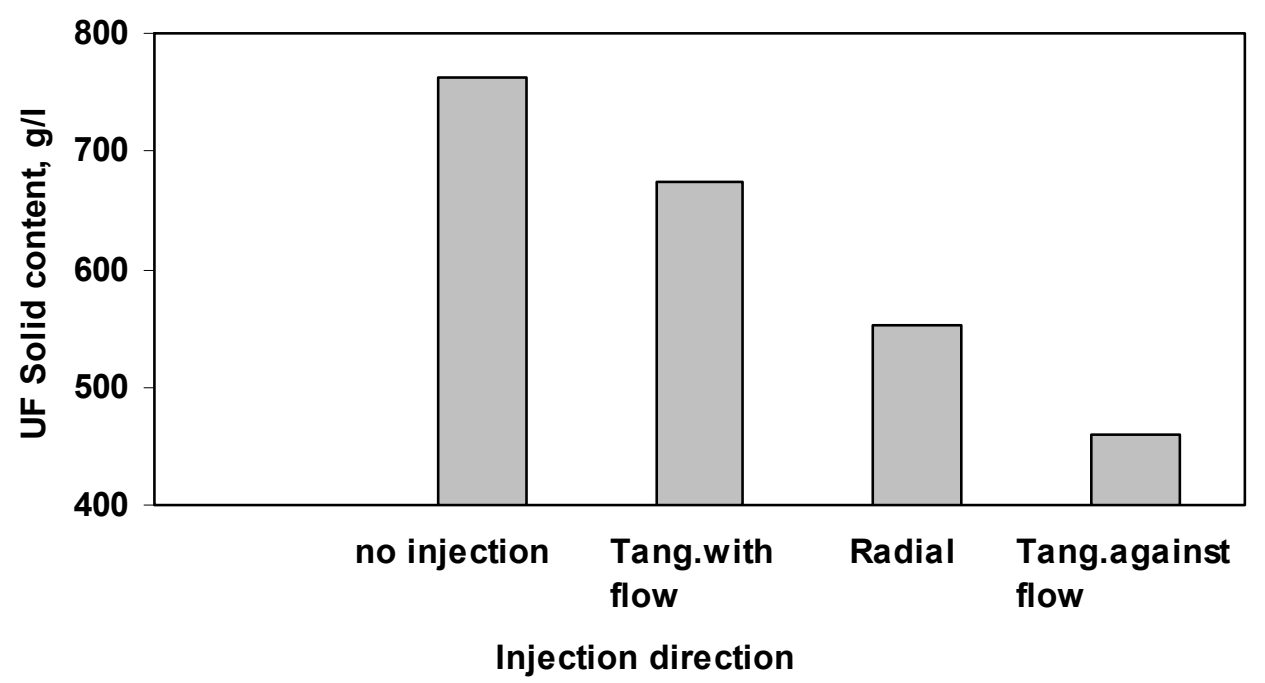

Fig. 7.12: Effect of injection direction on the underflow solid content.

Figure 7.12 show that the solid content in the underflow decreases slightly in case of tangential direction with the flow which may be due to the optimum effect of the injection that washes out only the fines from the underflow. The sharp decrease of the 
solid content in the case of radial and tangential against flow directions may be due to the turbulence occurred which causes more water goes to the underflow.

Figure 7.13 shows that the solid recovery in the underflow decreases in the case of tangential direction with the main flow. This may be due the transport of the fine particles by the injected water influence to the overflow and only the coarse particles will remain in the underflow discharge. The increase in solid recovery in the underflow in the other two directions is also expected, due to the high dilution of the underflow which reduces the underflow viscosity and therefore more suspension will flow through the apex.

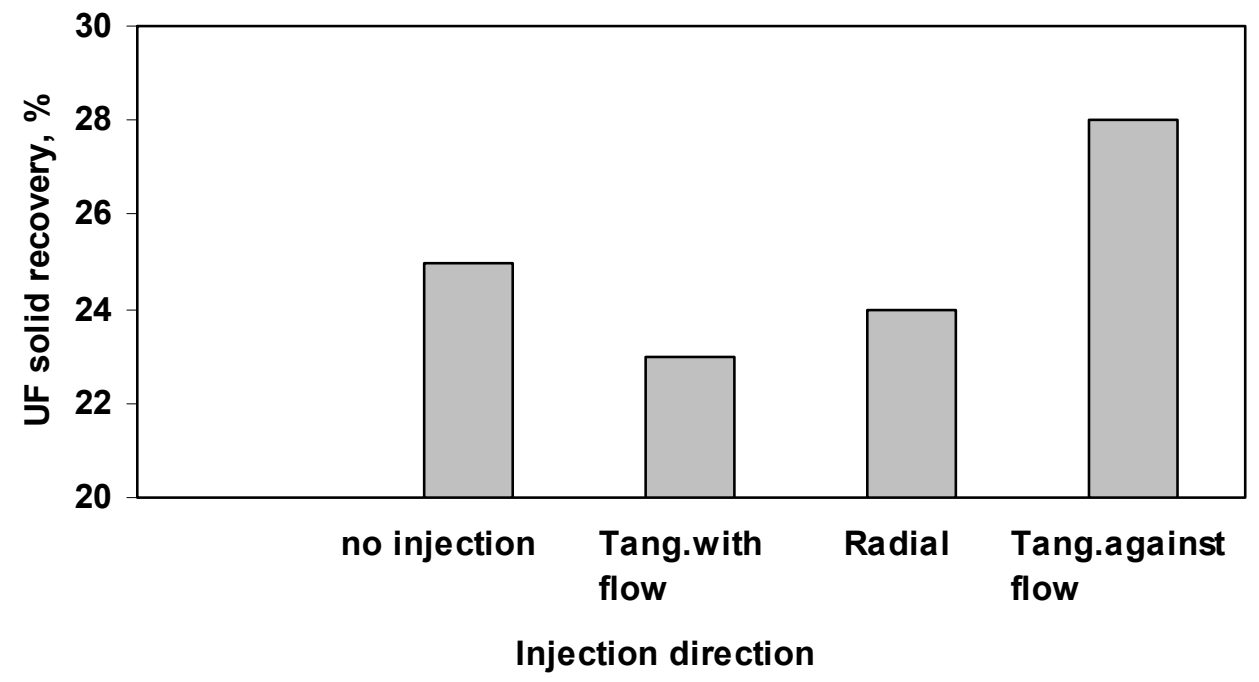

Fig. 7.13: Effect of injection direction on the underflow solid recovery.

\subsubsection{Effect of the Number of the Injection Openings}

\subsubsection{Effect of the Number of the Injection Openings on the Separation Efficiency of the Fine Particles $\left(\mathbf{T}_{0}, \mathbf{T}_{\min }\right)$}

To investigate the effect of the number of the injection openings, two injection rings were designed with three and five injection openings with the same opening diameter of $2.5 \mathrm{~mm}$.

Figure 7.14 shows the separation curves obtained using three and five injection openings. The change in the separation efficiency of the fine particles $\left(T_{0}, T_{\min }\right)$ with the number of injection openings is presented in Fig. 7.15. 


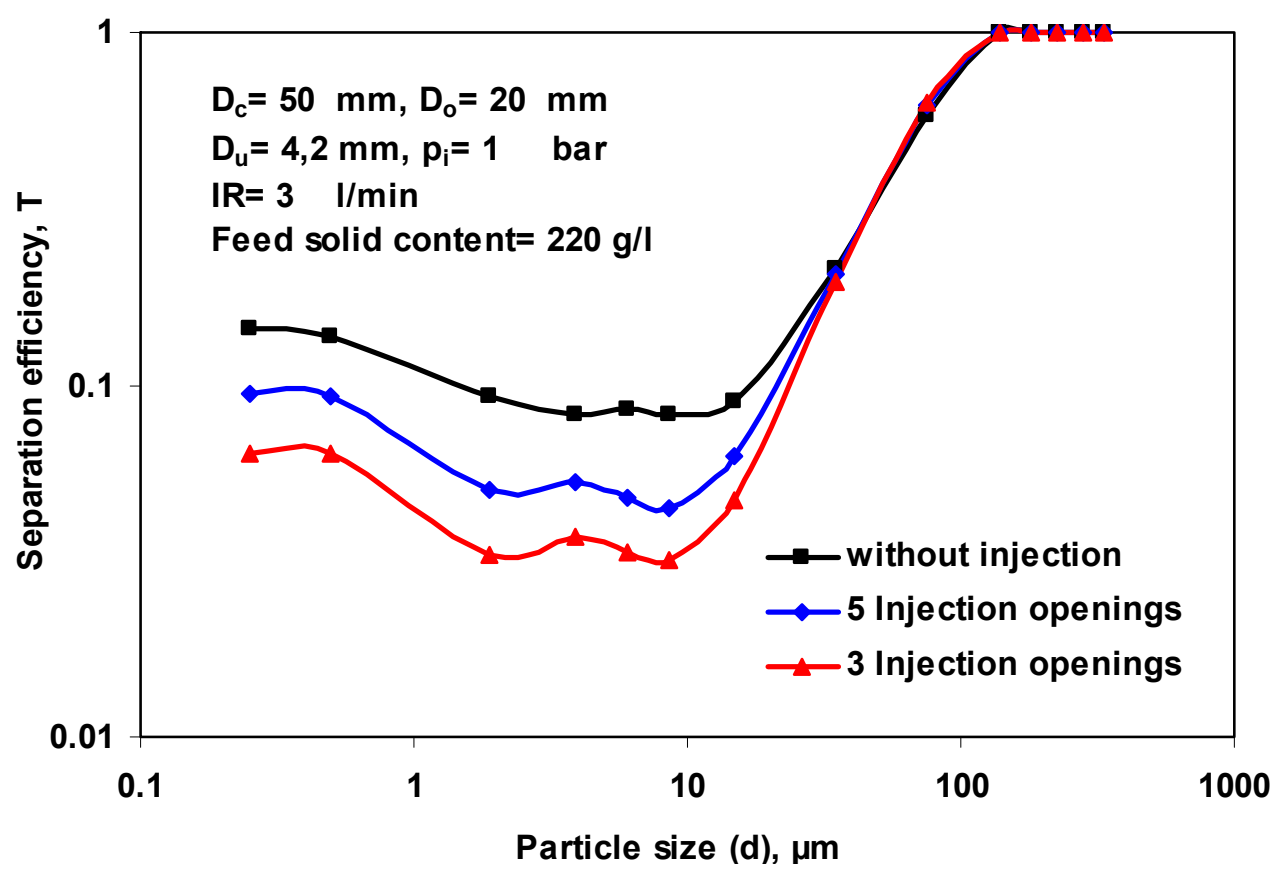

Fig. 7.14: Separation curves obtained using three and five injection openings.

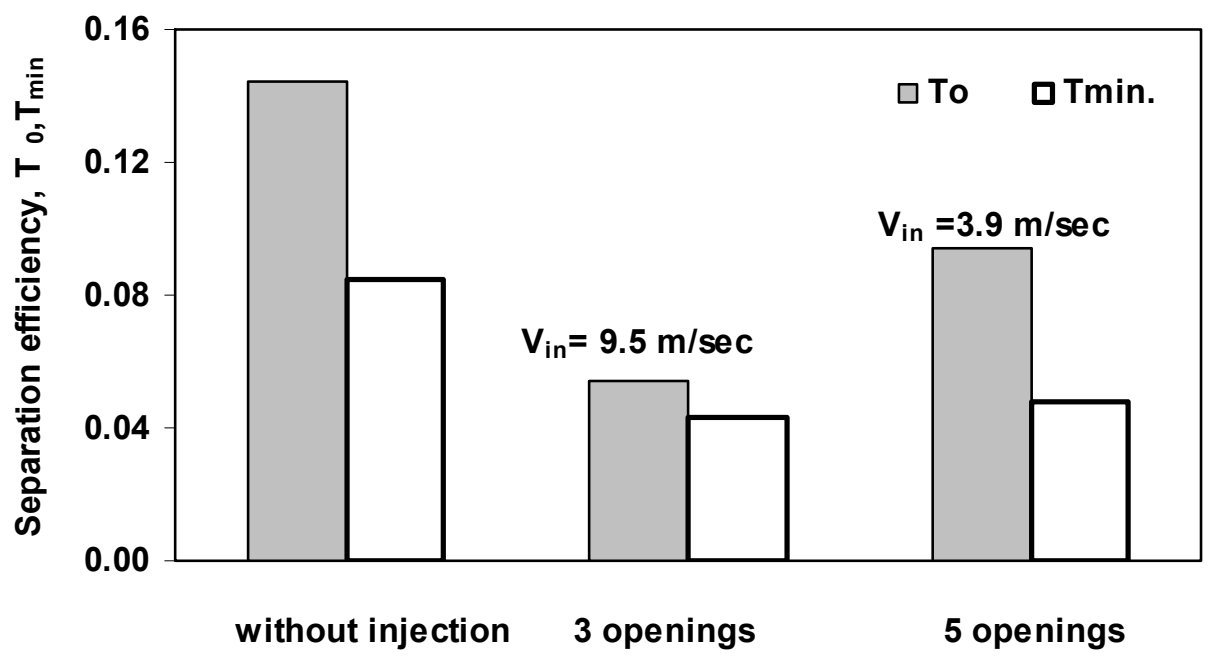

Number of injection openings

Fig. 7.15: Effect of the number of the injection openings on the separation efficiency of the fine particles $\left(\mathrm{T}_{0}, \mathrm{~T}_{\mathrm{min}}\right)$.

As can be seen from Fig. 7.14 and Fig. 7.15, the $T_{0}$ value achieved using three injection openings is lower than $T_{0}$ value obtained using five injection openings. This may be due to the fact that the higher injection velocity in the case of three openings 
$(9.5 \mathrm{~m} / \mathrm{sec})$ than the injection velocity in the case of five openings $(3.9 \mathrm{~m} / \mathrm{sec})$ which are calculated using Eq. (7.7).

$V_{\text {in }}=\frac{I R}{A_{\text {in }}}=\frac{4 I R}{N \pi d_{\text {in }}^{2}}$

where, $V_{\text {in }}$ is the injection velocity $(\mathrm{m} / \mathrm{sec}), I R$ is the injection rate, $N$ is the number of the injection openings, and $d_{i n}$ is the injection opening diameter (mm). For constant $I R$ and constant $d_{i n}$,Eq. (7.7) can be rewritten as follows:

$V_{\text {in }}=$ const $* \frac{1}{N}$.

According to Eq. (7.8) and Eq. (4.16), the $T_{0}$ value should be decreased by increasing the injection velocity (decreasing the number of the injection openings).

\subsubsection{Effect of the Number of the Injection Openings on the Cut Size $\left(d_{50}\right)$ and Imperfection (I).}

Figure 7.16 and Fig. 7.17 show the effect of changing the number of the injection openings on the cut size $\left(\mathrm{d}_{50}\right)$ and imperfection (I).

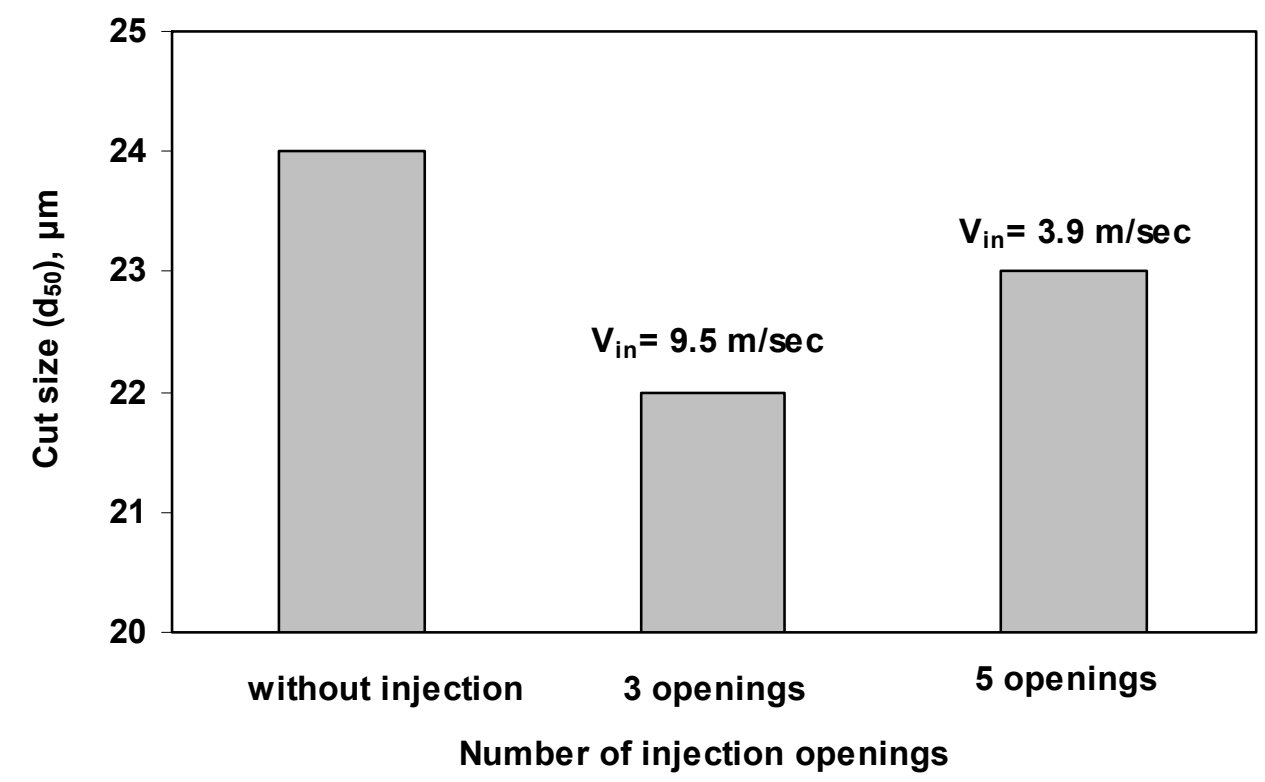

Fig. 7.16: Effect of the number of the injection openings on the cut size $\left(\mathrm{d}_{50}\right)$. 
Figure 7.16 shows that the cut size $\left(d_{50}\right)$ is decreased slightly in both cases of 3 and 5 injection openings compared with the case of no injection. This may be due to the injection effect where both have the same optimum injection direction. From Fig. 7.16 , it can be seen that the difference in the cut size $\left(\mathrm{d}_{50}\right)$ in the two cases is small. This means that the difference in the injection velocity may cause only more fines to be separated in the overflow and not affect the coarse particles. This may also be supported with the imperfection values shown in Fig. 7.17, where no change in the imperfection values between the two cases is observed.

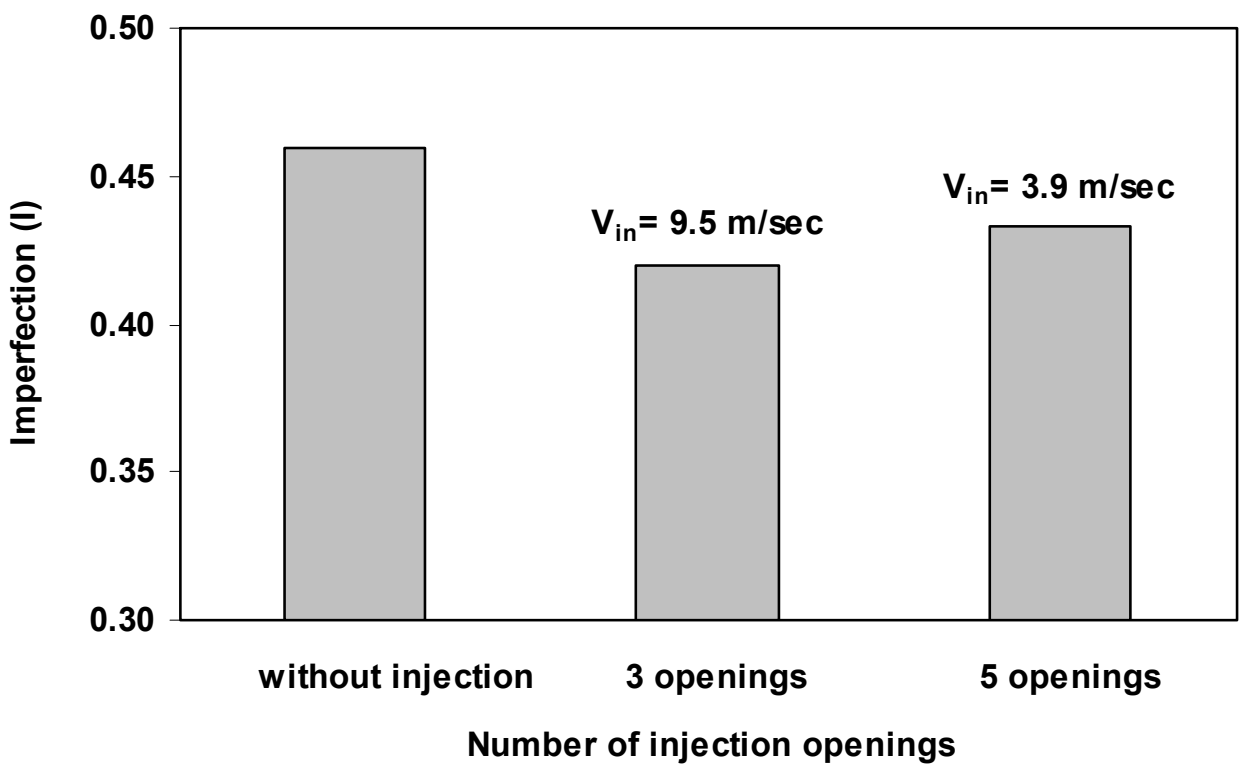

Fig. 7.17: Effect of the number of the injection openings on the imperfection (I).

\subsubsection{Effect of the Number of the Injection Openings on the Solid Recovery and Solid \\ Content}

Figure 7.18 and Fig. 7.19 show the change in the underflow solid recovery and underflow solid content as a result of changing the number of the injection openings. 


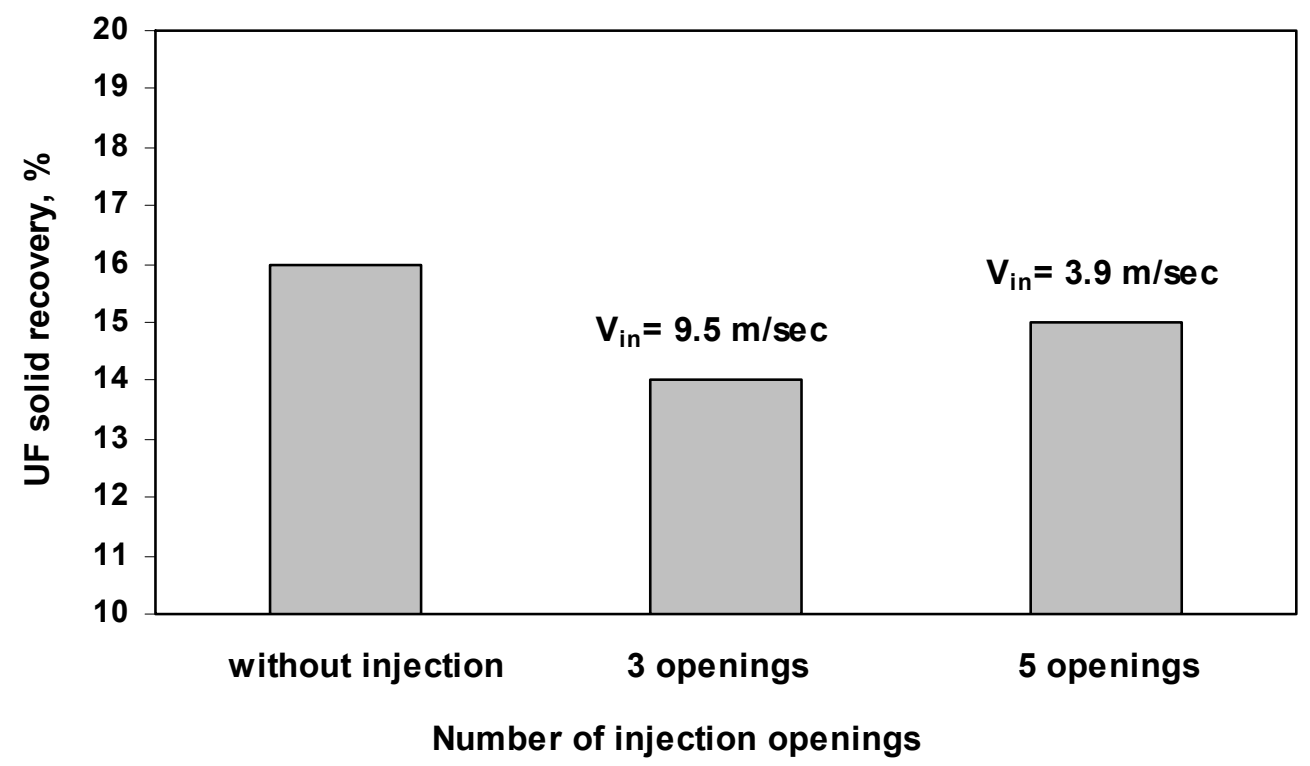

Fig. 7.18: Effect of the number of the injection openings on the underflow solid recovery.

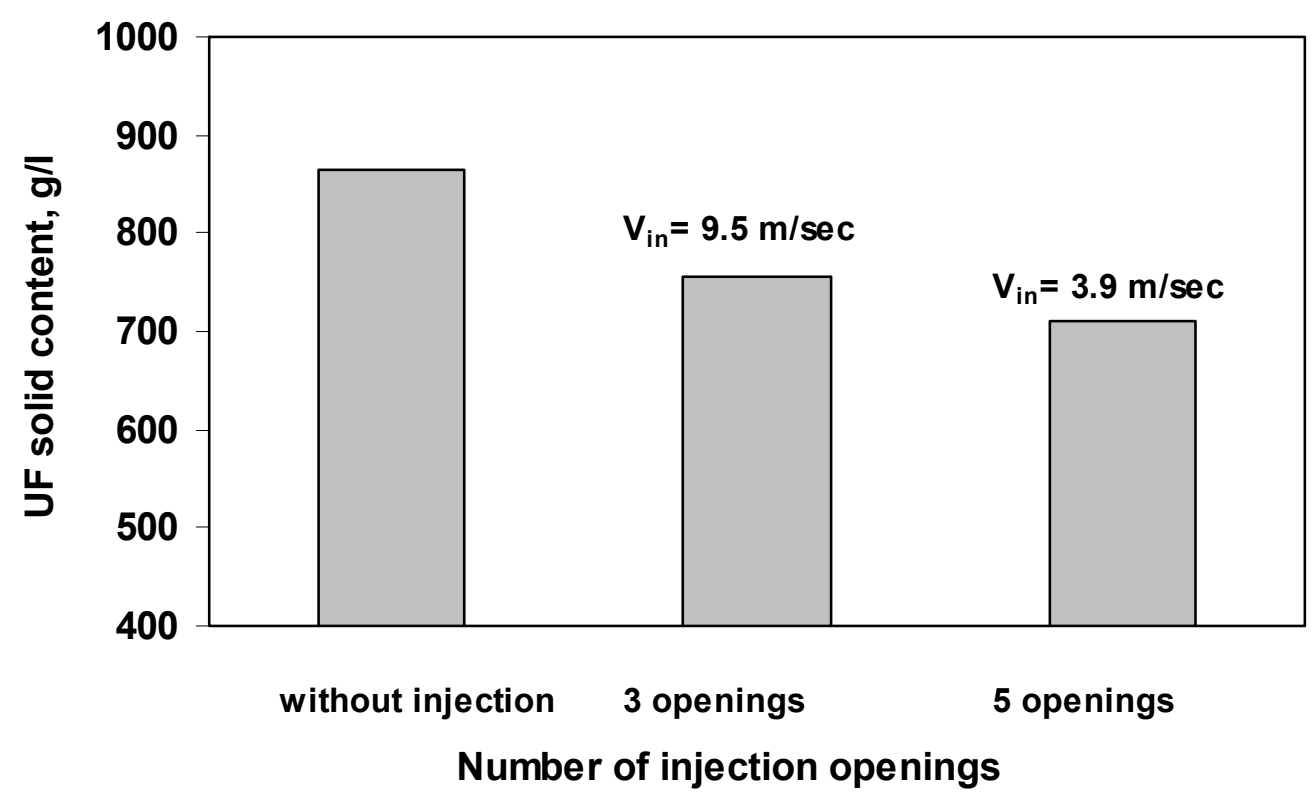

Fig. 7.19: Effect of the number of the injection openings on underflow solid content (g/l).

In the case of five injection openings, it was previously shown by Eq. (7.7) and Eq. (4.16) that the injection velocity is lower in the case of 5 injection openings than in the case of three injection openings. So, the whole injection stream can not across the locus of the zero axial velocity to reach the overflow vortex (the role of the locus will be discussed in details in the next chapter). This may cause more water to go to the 
underflow together with small amount of fines. This leads to an increase in the solid recovery and at the same time dilutes the underflow which results in low solid content. Therefore, it can be concluded that in the case of five injection openings, the injected water leads mainly to a high dilution in the underflow.

\subsubsection{Effect of Injection Opening Diameter $\left(d_{i n}\right)$.}

\subsubsection{Effect of Injection Opening Diameter on the Separation Efficiency of the} Fine

\section{Particles $\left(\mathbf{T}_{\mathbf{0}}, \mathbf{T}_{\min }\right)$.}

Figure 7.20 and Fig. 7.21 show the effect of the injection opening diameter $\left(\mathrm{d}_{\mathrm{in}}\right)$ at constant injection rate on the separation efficiency values. These figures show that the $T_{0}$ value decreases by decreasing the $\left(\mathrm{d}_{\text {in }}\right)$. This should be due to the increase in the injection velocity $(6.3 \mathrm{~m} / \mathrm{sec})$ in the case of the smaller diameter $(1.5 \mathrm{~mm})$ which is higher than the injection velocity $(2.9 \mathrm{~m} / \mathrm{sec})$ in the case of the large diameter $(2.5$ $\mathrm{mm}$ ). The effect of the injection velocity was previously discussed in detail.

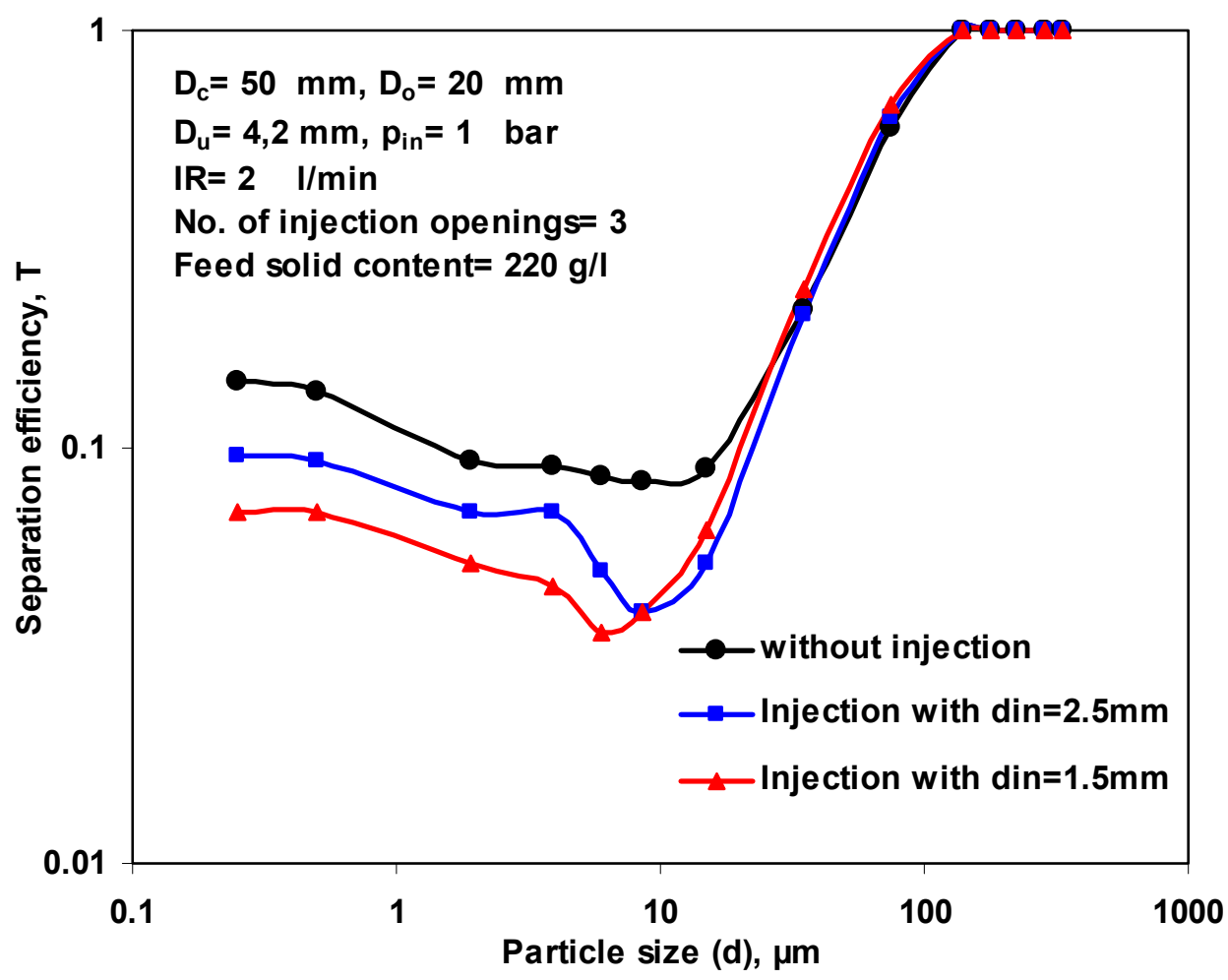

Fig. 7.20: Different separation curves at different injection opening diameters $\left(d_{\text {in }}\right)$. 


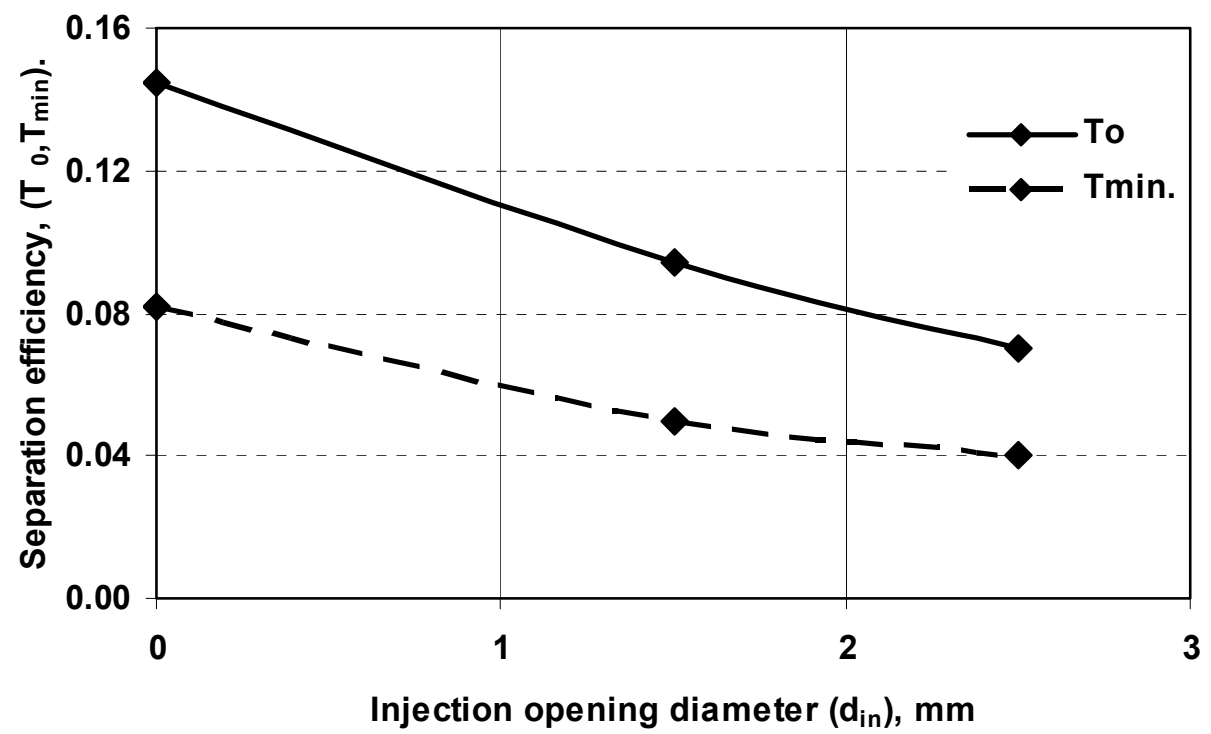

Fig. 7.21: Effect of injection opening diameter $\left(\mathrm{d}_{\mathrm{in}}\right)$ on the separation efficiency of the fine particles $\left(\mathrm{T}_{0}, \mathrm{~T}_{\min }\right)$.

\subsubsection{Effect of Injection Opening Diameter on the Cut Size $\left(d_{50}\right)$ and Imperfection (I).}

As can be seen from Fig.7.22 the cut size $\left(d_{50}\right)$ decreases slightly in both cases. This may be due to the injection influence together with the injection velocity effect which increases with decreasing the injection diameter. Decreasing the injection diameter $(1.5 \mathrm{~mm})$ must have the same effect as decreasing the injection openings number (3 openings) keeping all other conditions constant. This can be clearly seen from Figures 7.16 and 7.22 . 


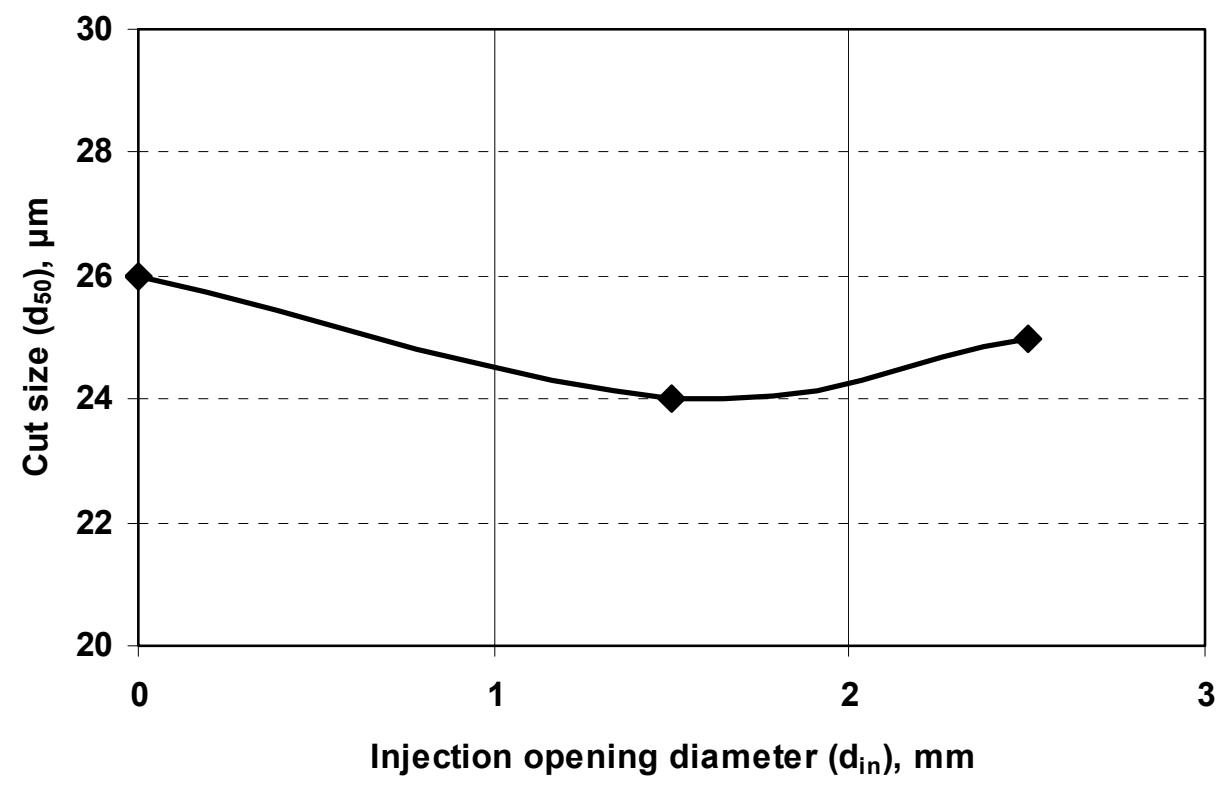

Fig. 7.22: Effect of injection opening diameter $\left(d_{i n}\right)$ on the cut size $\left(d_{50}\right)$.

Figure 7.23 shows that the imperfection value decreases by the injection effect and there is no big difference in both diameters.

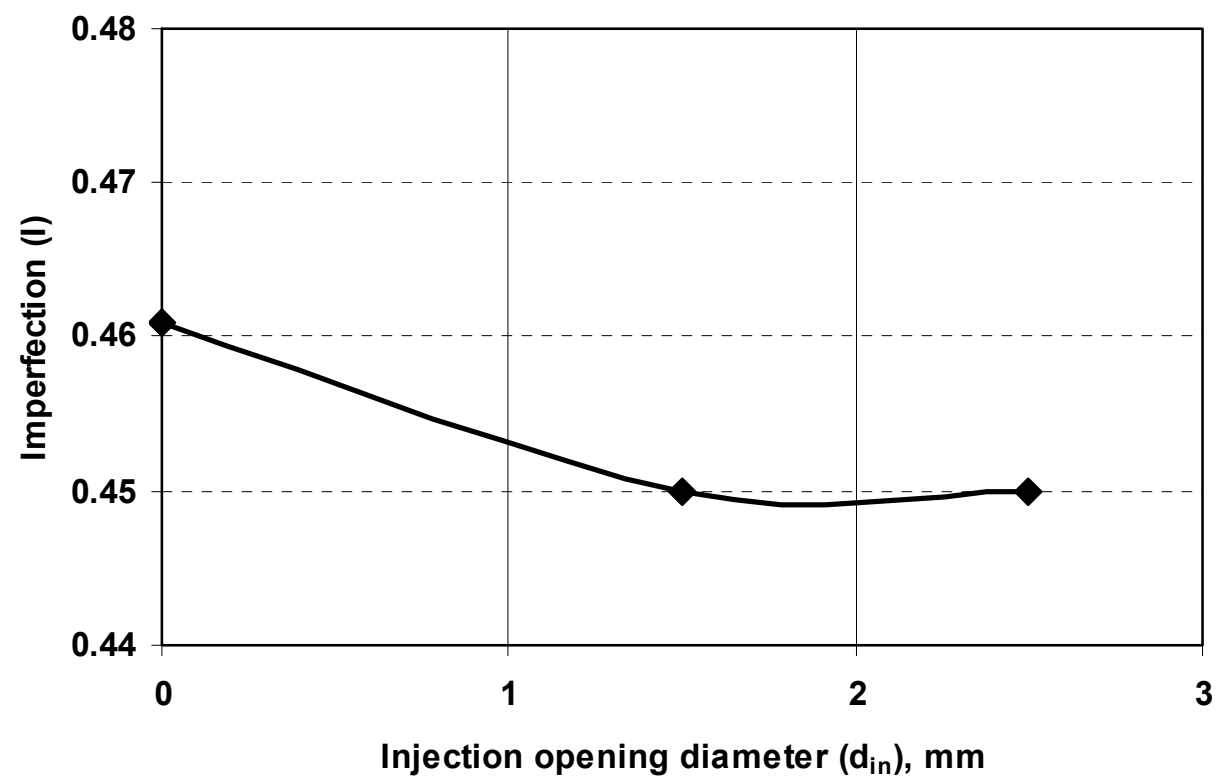

Fig. 7.23: Effect of injection openings diameter $\left(\mathrm{d}_{\text {in }}\right)$ on the imperfection $(\mathrm{I})$.

\subsubsection{Effect of Injection Opening Diameter on the Solid Content and Solid Recovery}

The effect of the injection opening diameter on the underflow solid content and the solid recovery is shown in Figures 7.24 and 7.25. It could be expected that the change 
in the injection opening diameter has the same effect as the change in the number of injection openings which is discussed previously. The solid content decreased and the solid recovery increased as the injection opening diameter increased (Fig. 7.24, Fig. 7.25).

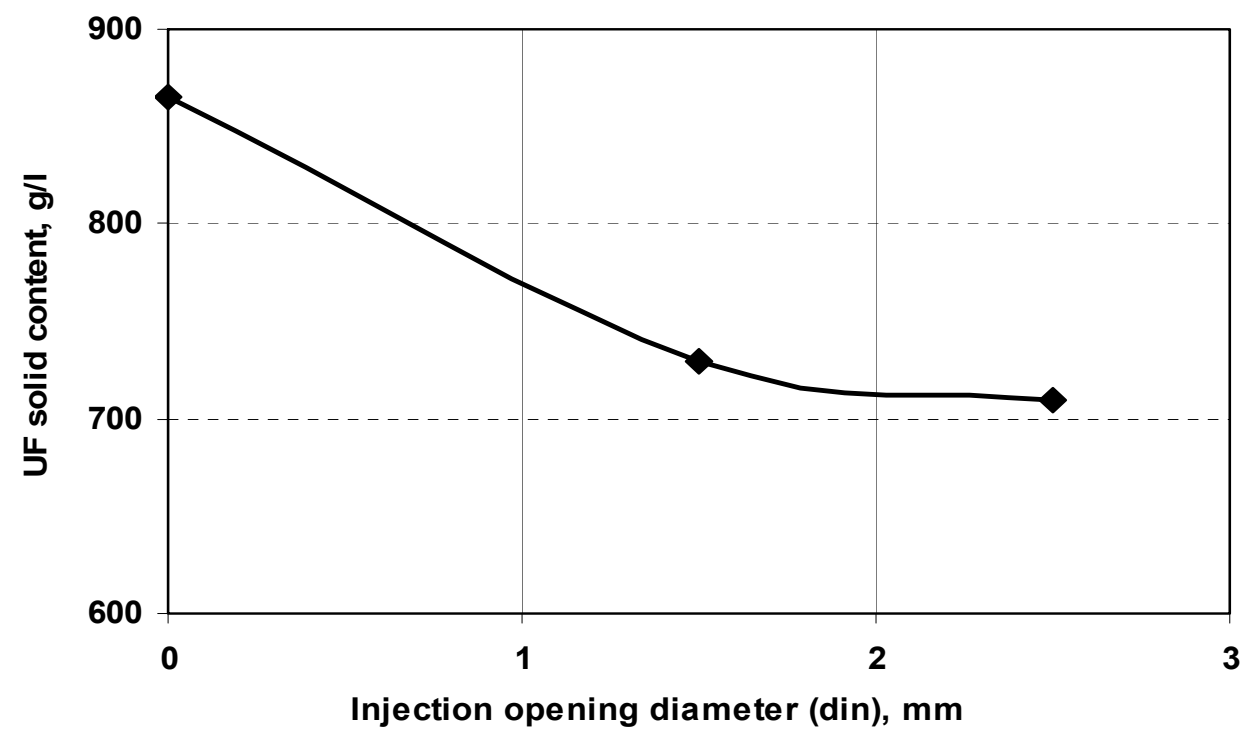

Fig. 7.24: Effect of injection opening diameter $\left(\mathrm{d}_{\text {in }}\right)$ on the underflow solid content $(\mathrm{g} / \mathrm{l})$.

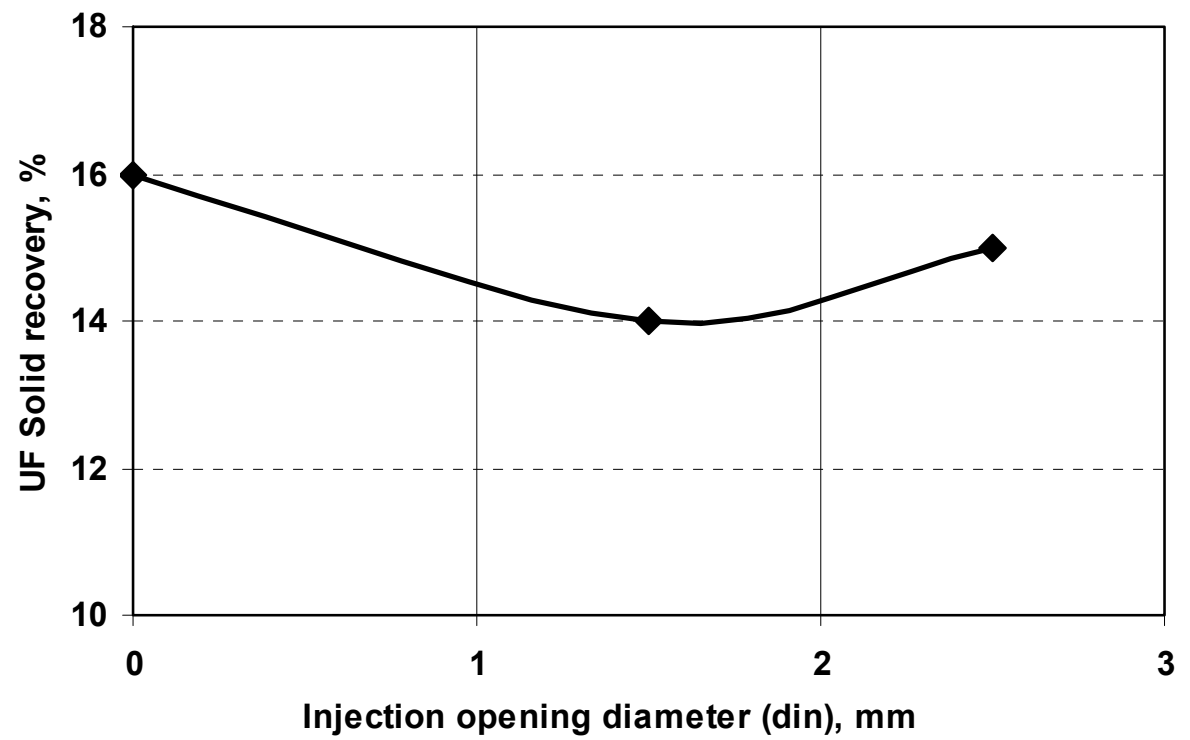

Fig. 7.25: Effect of injection opening diameter $\left(\mathrm{d}_{\mathrm{in}}\right)$ on the underflow solid recovery. 


\subsubsection{Effect of The Injection Height From The Apex (h)}

\subsubsection{Effect of Injection Height on the Separation Efficiency of the Fine Particles}

$$
\left(\mathbf{T}_{\mathbf{0}}, \mathbf{T}_{\mathrm{min}}\right)
$$

The injection position is of a great importance to achieve the optimum injection effect. In the present work, the injection was installed at three different injection heights from the upper end of the apex. These heights were at $40 \mathrm{~mm}$, at $80 \mathrm{~mm}$, and at120 mm. Figure 7.26 shows the difference in the separation curve obtained at the different injection heights from the apex. The effect of the injection height on the separation efficiency values is shown in Fig. 7.27.

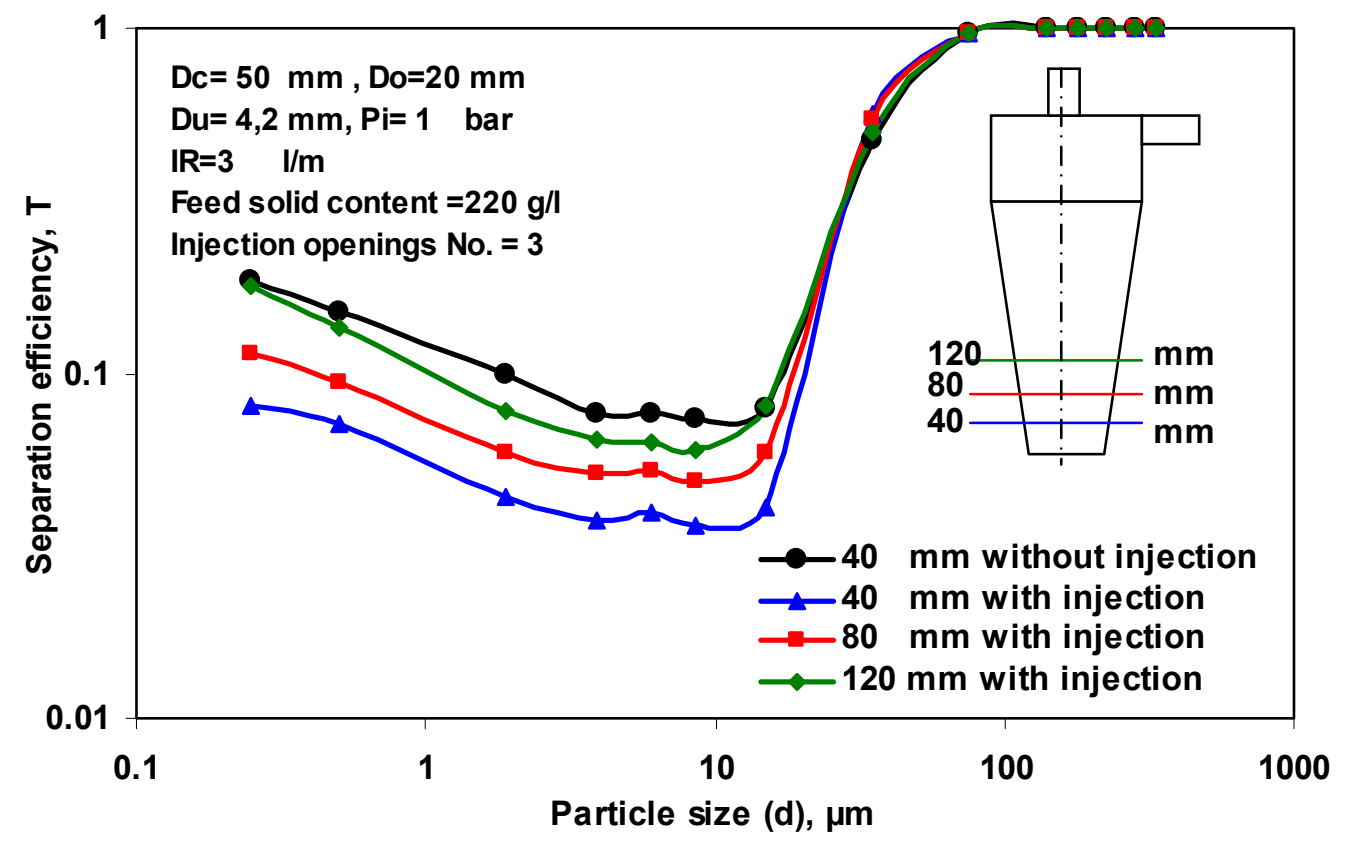

Fig. 7.26: Different separation curves at different injection heights from the apex. 


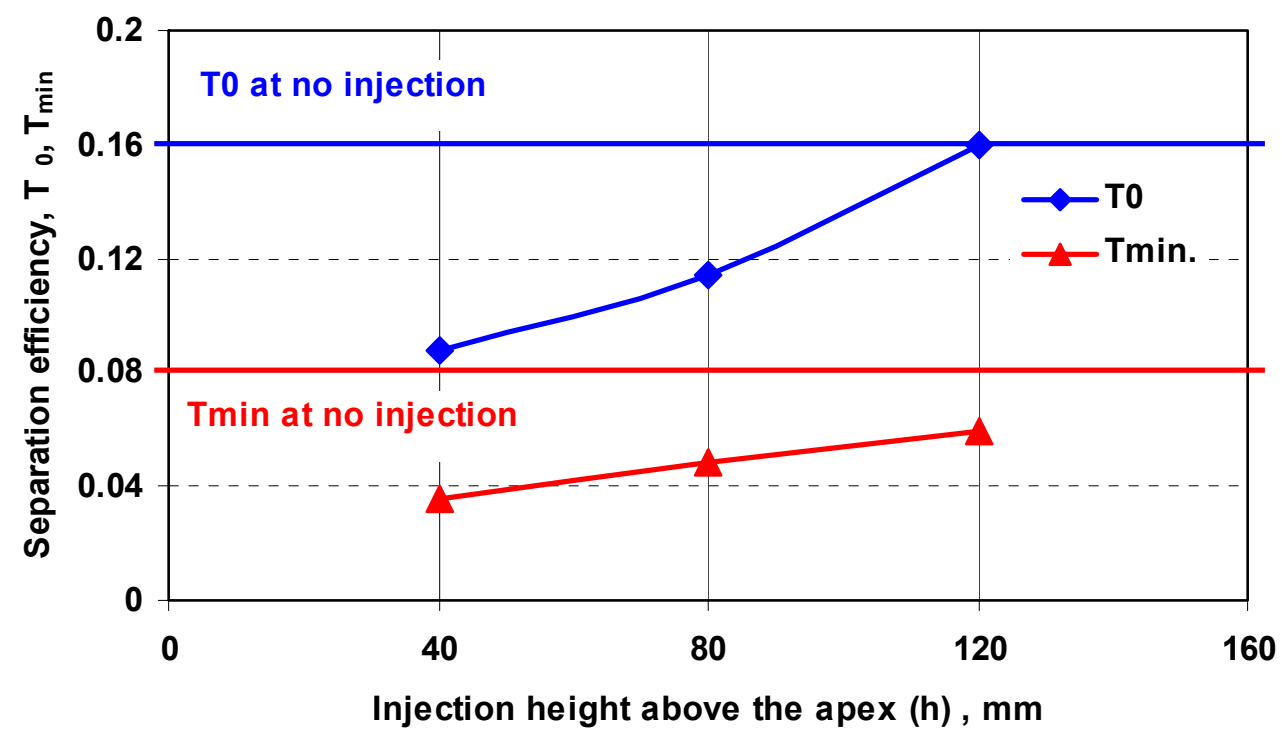

Fig. 7.27: Effect of injection height (h) on the separation efficiency of the fine particles $\left(\mathrm{T}_{0}, \mathrm{~T}_{\min }\right)$.

From Fig. 7.26 and Fig. 7.27, it can be shown that the lowest $T_{0}$ value is achieved at the lower injection height $(40 \mathrm{~mm})$ and then increases with increasing the injection height. At high injection height $(120 \mathrm{~mm})$, the $T_{0}$ value is the same as without injection which means that no injection effect occurs at this height. This can be explained based on the locus of the zero axial velocity inside the hydrocyclone. According to Kelsall [43], Bloor et al. [101], and Laverack [102], inside this locus, the direction of the axial velocity is upward and all particles inside this zone will go to the vortex finder and then discharged in the overflow. Outside this locus and towards the wall of the cyclone, the fluid spiral moves downwards which means that the axial velocity is downwards and all the particles in this area tend to move to the apex and are discharged in the underflow. Figure 7.28 shows the locus of zero axial velocity together with the different injection heights. As can be seen, at low injection height, the radial distance between the injected position and the air core surface (R1) is short. So the injected water can easily cross the locus of zero axial velocity to go through the overflow with the washed out fines. By increasing the injection height, the axial distance (R2) and (R3) increase, and the injected water just partially crosses the locus and most of it will be entrained by the main flow and go through the underflow discharge. 


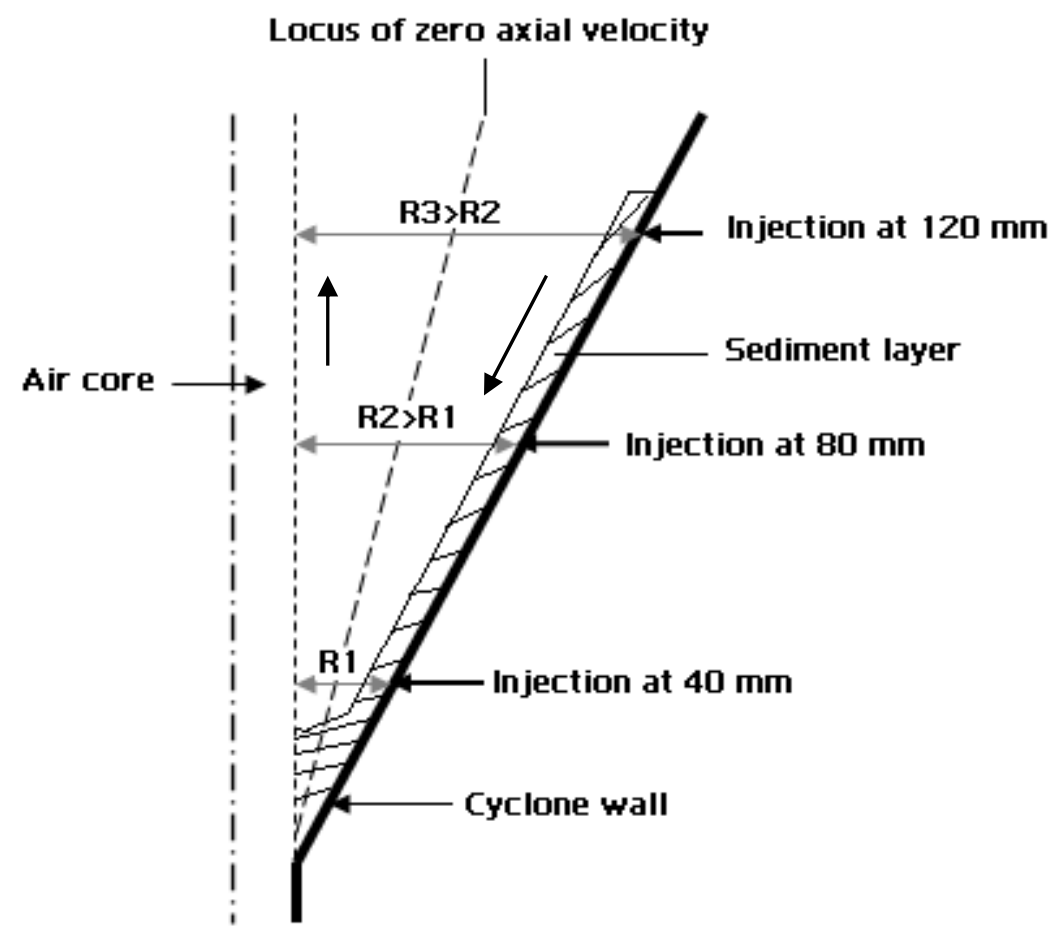

Fig. 7.28: Locus of zero axial velocity after Kelsall [43], together with the different injection heights.

\subsubsection{Effect of Injection Height on the Cut Size $\left(d_{50}\right)$ and Imperfection (I).}

Figure 7.29 shows that the cut size $\left(\mathrm{d}_{50}\right)$ decreases at the lowest injection point (40 $\mathrm{mm})$. Even at the higher injection height the cut size is slightly lower than without injection.

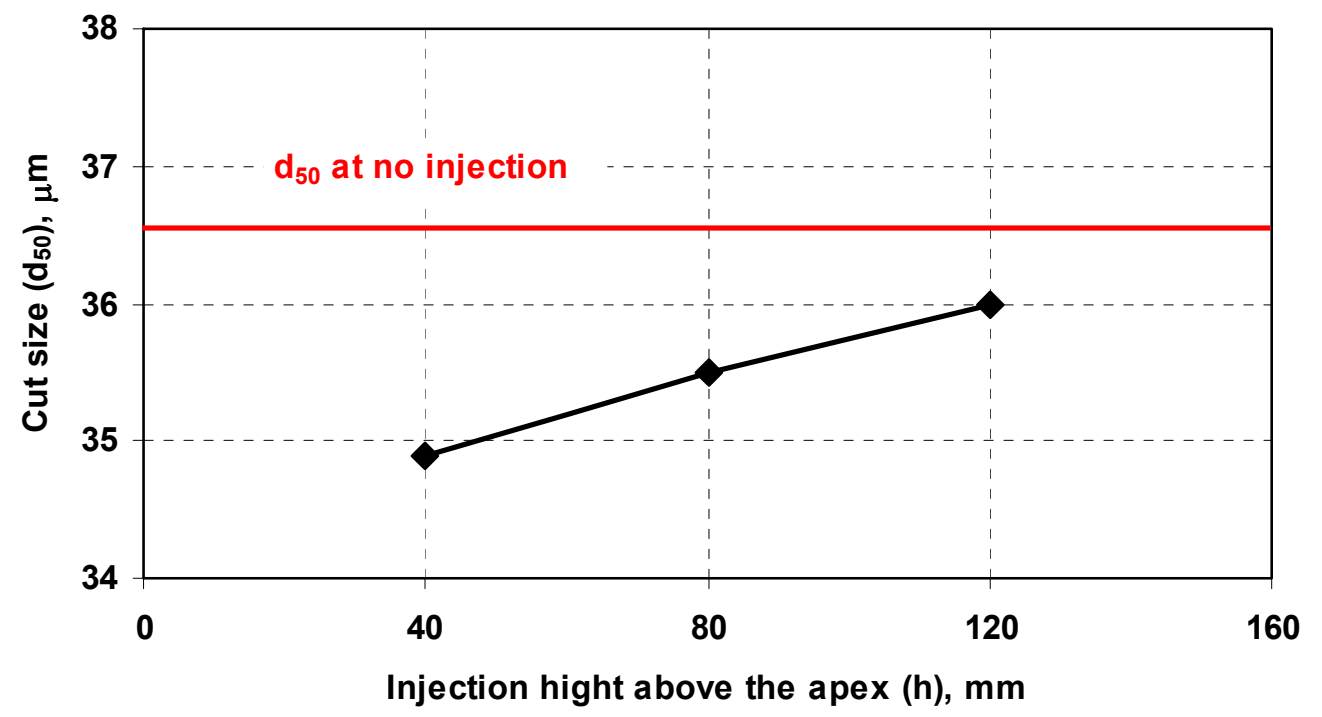

Fig. 7.29: Effect of injection height $(\mathrm{h})$ on the cut size $\left(\mathrm{d}_{50}\right)$. 
This can be explained based on Eq. (4.18) according to the dilution of the underflow (low solid content) and the increased tangential velocity which enables better settling conditions for the coarse particles. This leads to lower cut size and better imperfection values as it can be seen from Fig. 7.30.

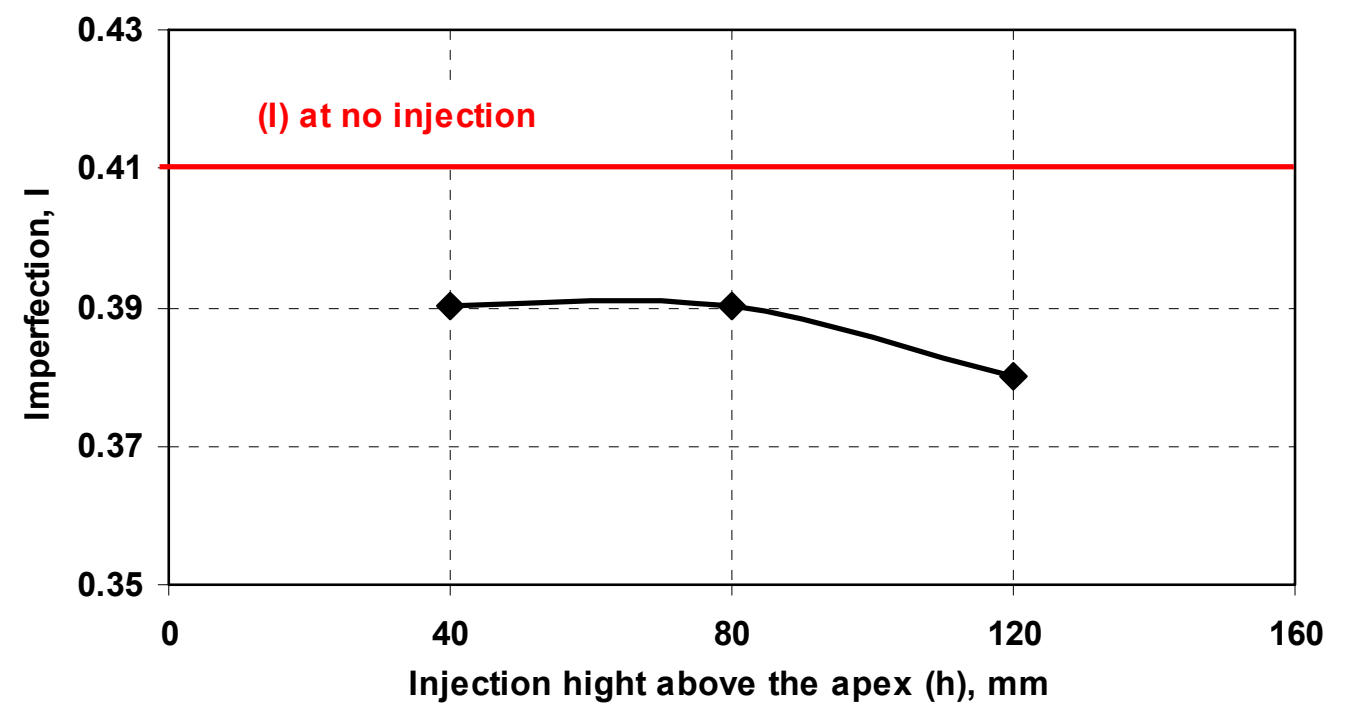

Fig. 7.30: Effect of injection height (h) on the imperfection (I).

\subsubsection{Effect of Injection Height on the Solid Recovery and Solid Content}

The variation of the solid recovery and the solid content in the underflow at different injection heights can be seen in Fig. 7.31 and Fig. 7.32, respectively.

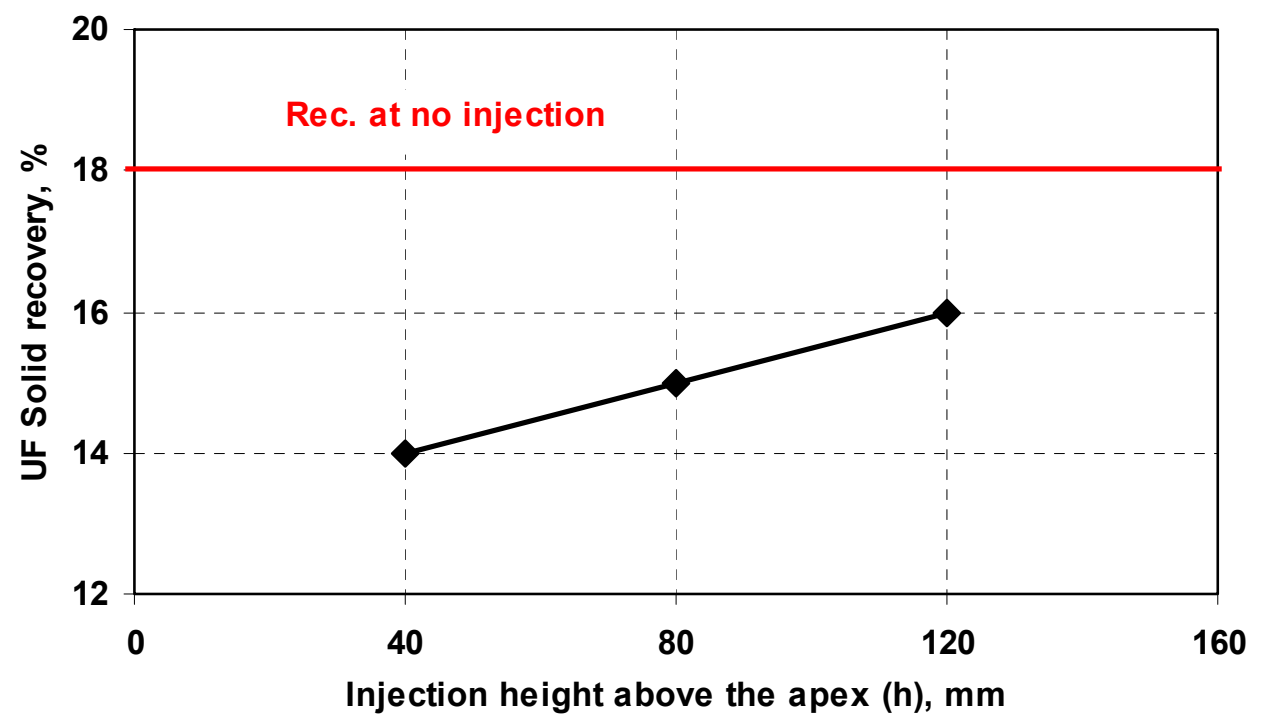

Fig. 7.31: Effect of injection height (h) on the underflow solid recovery. 
The decrease in the solid recovery at the lower injection height $(40 \mathrm{~mm})$ can be explained according to Fig. 7.28 where the radial distance of the injection (R1) is small, which causes more injected water to cross the locus of zero axial velocity carrying more fines (solids) from the underflow to the overflow product. The increase in the solid recovery in the other two heights could be expected due to the increase in the radial distances $\mathrm{R} 2$ and $\mathrm{R} 3$ at these two positions, which allows more solids to pass to the underflow.

Figure 7.32 shows that the solid content decreases gradually by increasing the injection height. This may be that at high levels, the water stream will not be able to cross the locus of the zero axial velocity and therefore more water will go to the underflow causing more dilution and low solid content.

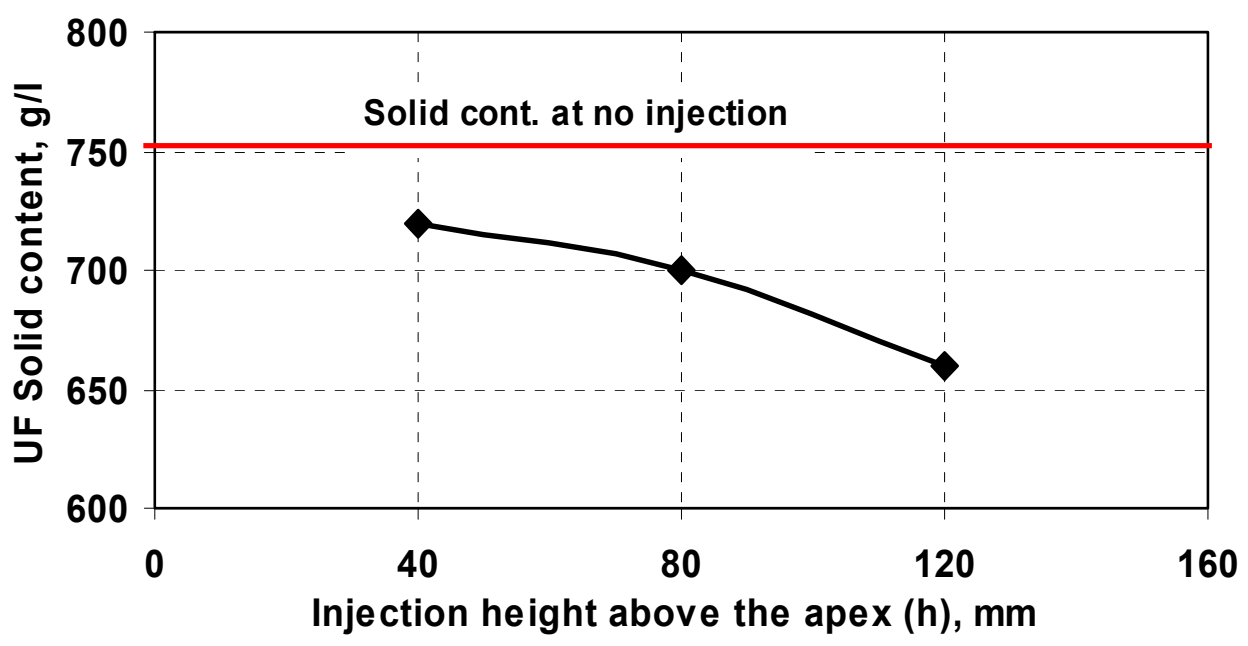

Fig. 7.32: Effect of injection height (h) on the underflow solid content $(\mathrm{g} / \mathrm{l})$.

\subsubsection{Effect of Inner Vortex Finder Length (IVFL)}

\subsubsection{Effect of Inner Vortex Finder Length on the Separation Efficiency of the Fine \\ Particles $\left(\mathbf{T}_{\mathbf{0}}, \mathbf{T}_{\min }\right)$.}

The vortex finder length plays an important role in the hydrocyclone separation. The vortex finder length should be long enough to guaranty a minimum residence time for the particles to settle out and not to be discharged directly to the overflow by the bypass flow. The existence of the vortex finder prevents the suction of the sediment 
particles to the overflow. However, the longer the vortex finder length, the more extension into the sediment zones where the intermediate and the underflow sizes are located. Therefore the vortex finder length should be optimized. According to Renner and Cohen [104], the particle size distribution inside the hydrocyclone can be classified into four different regions (A, B, C, and D) as can be seen from Fig. 7.33.
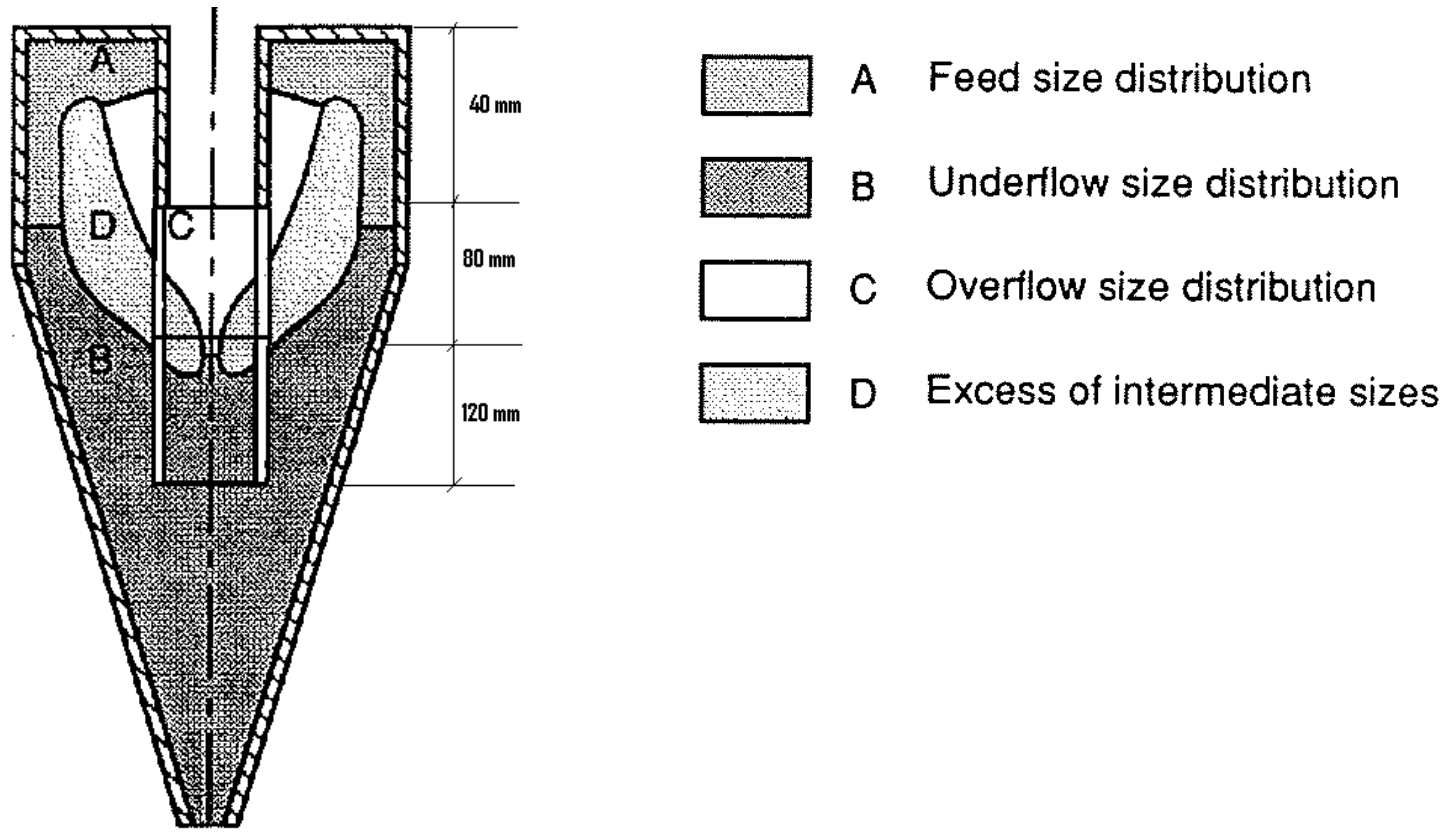

Fig. 7.33: Size distribution regions in a hydrocyclone, after Renner and Cohen [104].

So that, it was planned to change the vortex finder length to test the regions of the different sizes in the hydrocyclone (A, B, C, and D) and examine the washing effect at these different lengths. The experiments were carried out using three different inner vortex finder lengths of $40 \mathrm{~mm}$ (the normal length built-in the hydrocyclone), $80 \mathrm{~mm}$, and $120 \mathrm{~mm}$.

Figure 7.34 shows different separation curves obtained at different inner vortex finder lengths. Figure 7.35 shows the change in the separation efficiency of the fine particles $\left(\mathrm{T}_{0}, \mathrm{~T}_{\mathrm{min}}.\right)$ at different inner vortex finder lengths. 


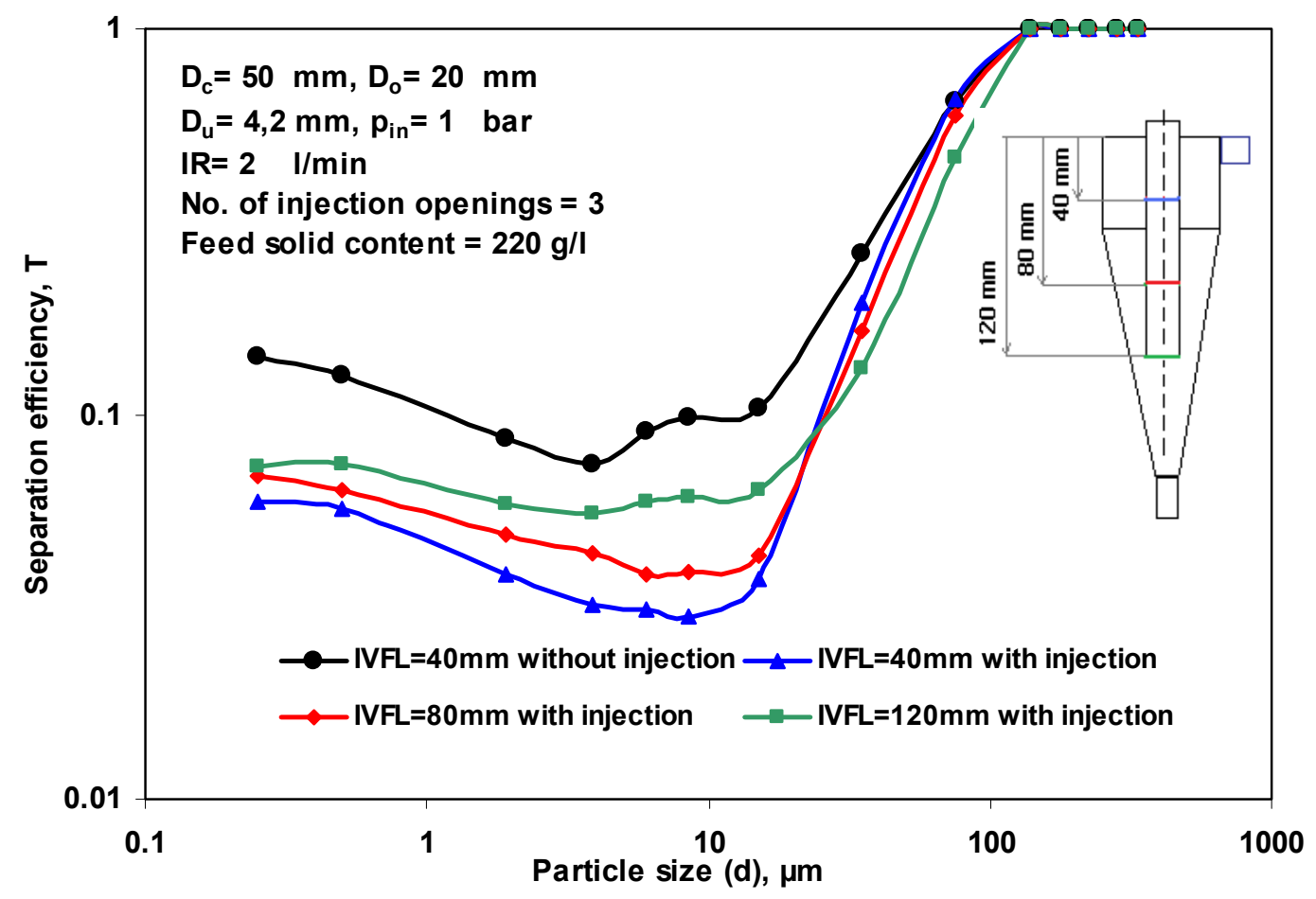

Fig. 7.34: Different separation curves obtained at different inner vortex finder lengths.

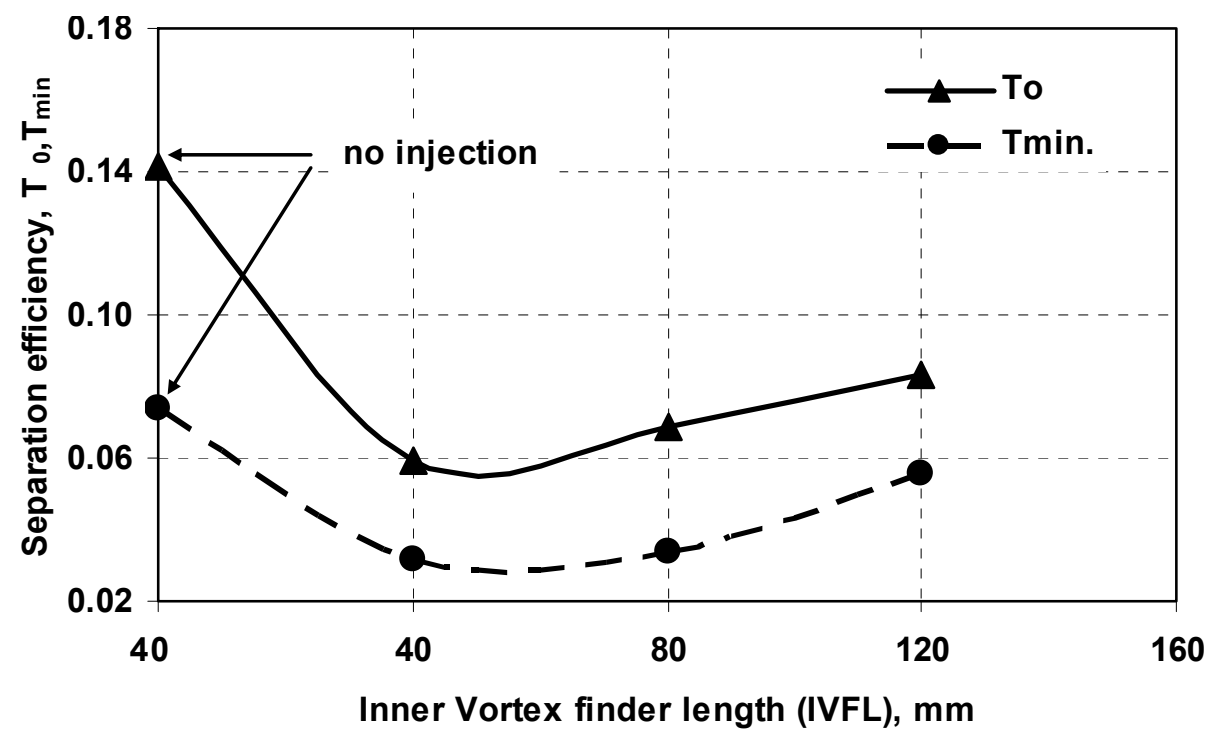

Fig. 7.35: Effect of changing the inner vortex finder length on the separation efficiency of the fine particles $\left(\mathrm{T}_{0}, \mathrm{~T}_{\mathrm{min}}\right)$.

From Fig. 7.34 and Fig. 7.35, it can be seen that $T_{0}$ decreases with water injection independent of the vortex finder length and the difference between the values of $T_{0}$ in 
the three different lengths is not high. This may be due to the injection effect which decreases the fines in the underflow.

\subsubsection{Effect of Inner Vortex Finder Length on the Cut Size $\left(d_{50}\right)$ and Imperfection (I).}

Figure 7.36 shows that the cut size $\left(\mathrm{d}_{50}\right)$ does not change at the lower IVFL and then increases sharply with increasing the IVFL.

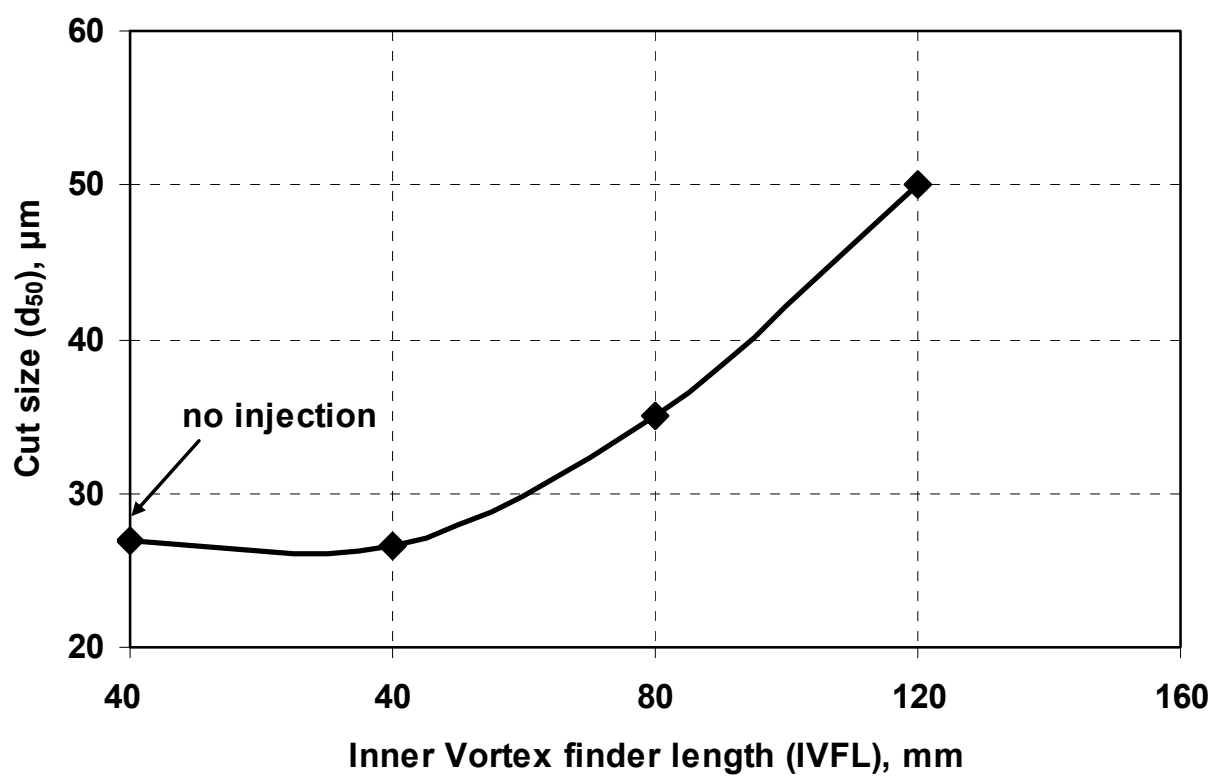

Fig. 7.36: Effect of changing the inner vortex finder length on the cut size $\left(\mathrm{d}_{50}\right)$.

This can be explained by Fig. 7.28 and Fig. 7.33. As can be seen from Fig. 7.28, by extending the vortex finder length downward, the locus of zero axial velocity comes closer to the wall where the coarse particles exist, which means that more coarse will move through the injection to the overflow causing higher cut sizes. Fig. 7.33 shows that by increasing the IVFL, the vortex finder will be more extended to the intermediate sizes region (D) and the underflow size distribution region (B) which results in higher cut sizes.

Figure 7.37 shows that the imperfection value decreases gradually by increasing the vortex finder length inside the hydrocyclone. The low imperfection value in the case of the small length $(40 \mathrm{~mm})$ may be due to the positive effect of the dilution caused by the injection. The decrease in the imperfection values in the case of the other two 
lengths may be due to the increase in the cut size $\left(\mathrm{d}_{50}\right)$ where the imperfection value is inversely proportional to $\mathrm{d}_{50}$ as shown in Eq. (1.2). Actually at $120 \mathrm{~mm}$, the imperfection will be worse if we consider the slope of the partition curve between the sizes $d_{25}$ and $d_{50}$. So, it should be noted that the definition of the imperfection in this case does not reflect the real conditions of the entire separation curve.

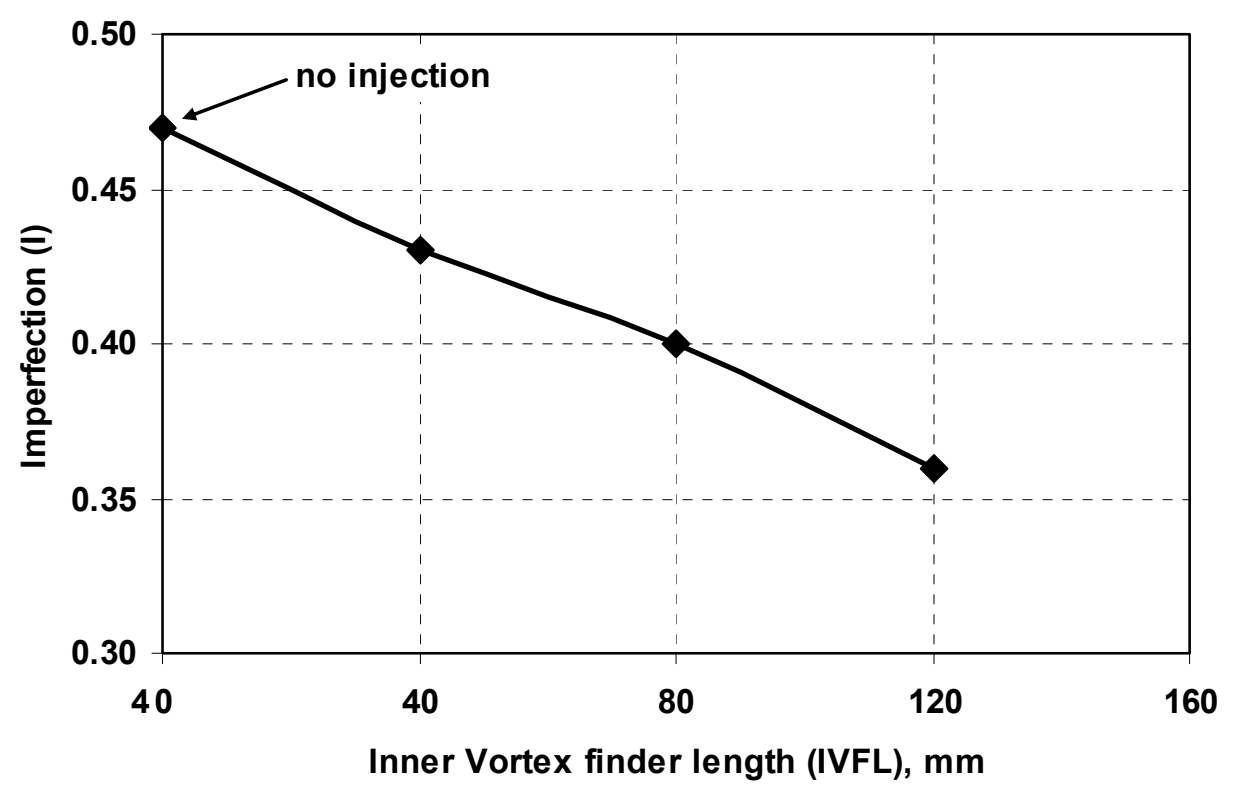

Fig. 7.37: Effect of changing the inner vortex finder length on the imperfection (I).

\subsubsection{Effect of Inner Vortex Finder Length on the Solid Recovery and Solid Content}

Figure 7.38 shows that the solid recovery in the underflow decreases contentiously with increasing the vortex finder length inside the hydrocyclone. 


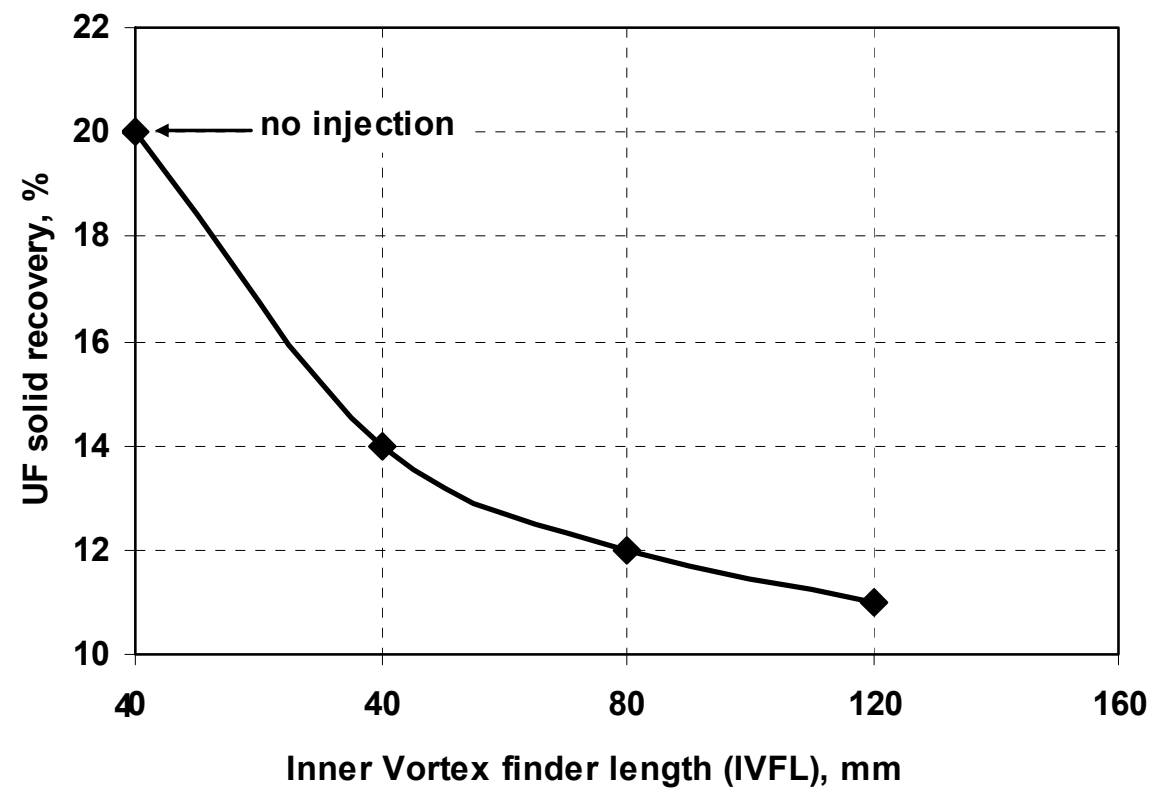

Fig. 7.38: Effect of changing the inner vortex finder length on the underflow solid recovery.

This may be due to the extension of the vortex finder in the coarse particles region as it can be seen in Fig. 7.28 and Fig. 7.33, so that more solids report to the overflow, in stead of going to the underflow. This leads to lower solid recovery and more diluted underflow discharge, which results in lower solid content as it can be seen from Fig. 7.39 .

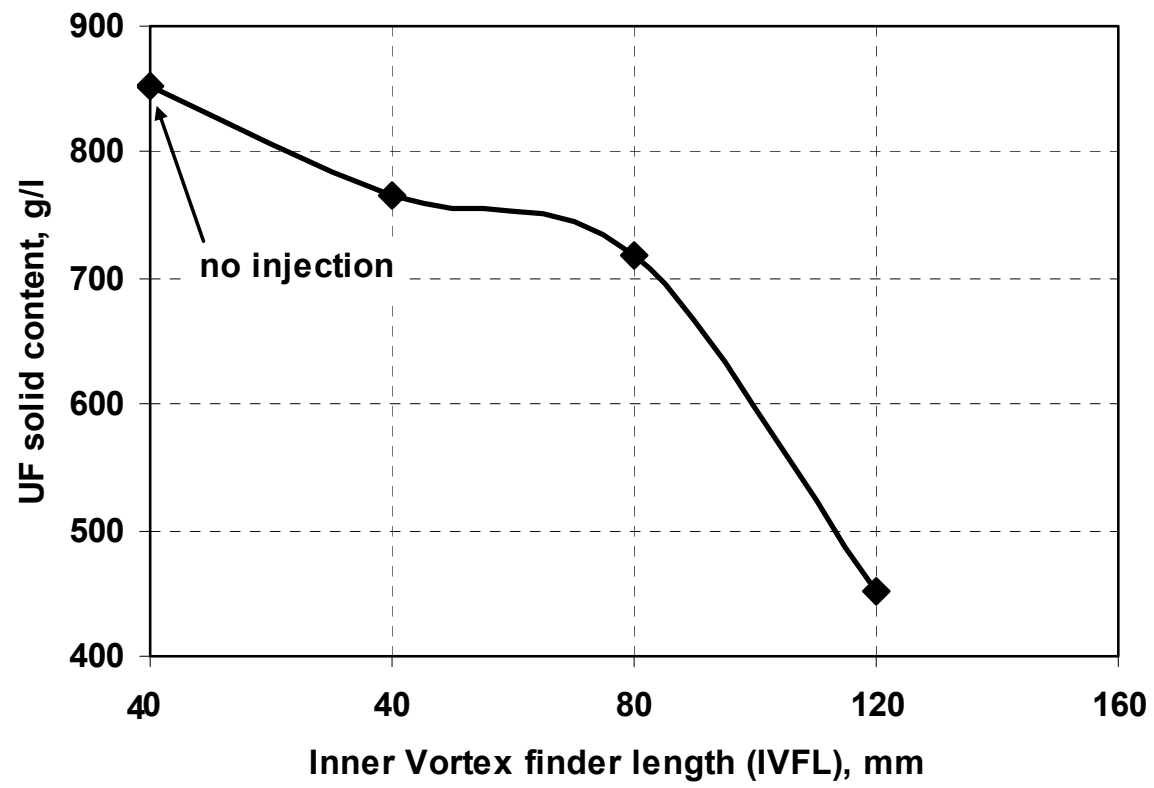

Fig. 7.39: Effect of changing the inner vortex finder length on the underflow solid content $(\mathrm{g} / \mathrm{l})$. 
From the above discussion, it can be seen that the extension of the inner vortex finder length beyond the normal designed length of the hydrocyclone does not provide any positive effect concerning the injection influence.

\subsubsection{Effect of Injection Rate (IR) and Injection Velocity ( $\left.V_{\text {in }}\right)$.}

Injection rate is a key variable in this study. Therefore, the effect of injection rate on the percentage fine in the underflow was investigated extensively. The proposed effect of the injection flow is to displace the feed water which goes to the underflow to move the suspension to the hydrocyclone centre as was previously shown in Fig. 4.1 .

The injection was expressed by the injection rate $(I R)$ in $1 / \mathrm{m}$ and the equivalent injection velocity $\left(V_{i n}\right)$ in $\mathrm{m} / \mathrm{sec}$. The injection velocity $\left(V_{i n}\right)$ was determined from Eq. (7.7). According to Eq. (7.7), the injection velocity can be varied by changing the number of the injection openings and /or the diameter of the injection openings. Therefore two injection rings with three and five injection openings were used to operate at low and high injection velocity ranges respectively.

\subsubsection{Effect of Low Injection Velocities}

Figure 7.40 shows the separation curves obtained at low injection velocities ranging from $0.7 \mathrm{~m} / \mathrm{sec}(1 \mathrm{l} / \mathrm{min})$ to $3.88 \mathrm{~m} / \mathrm{sec}(5.7 \mathrm{l} / \mathrm{min})$. These values are obtained when 5 injection openings are used. 


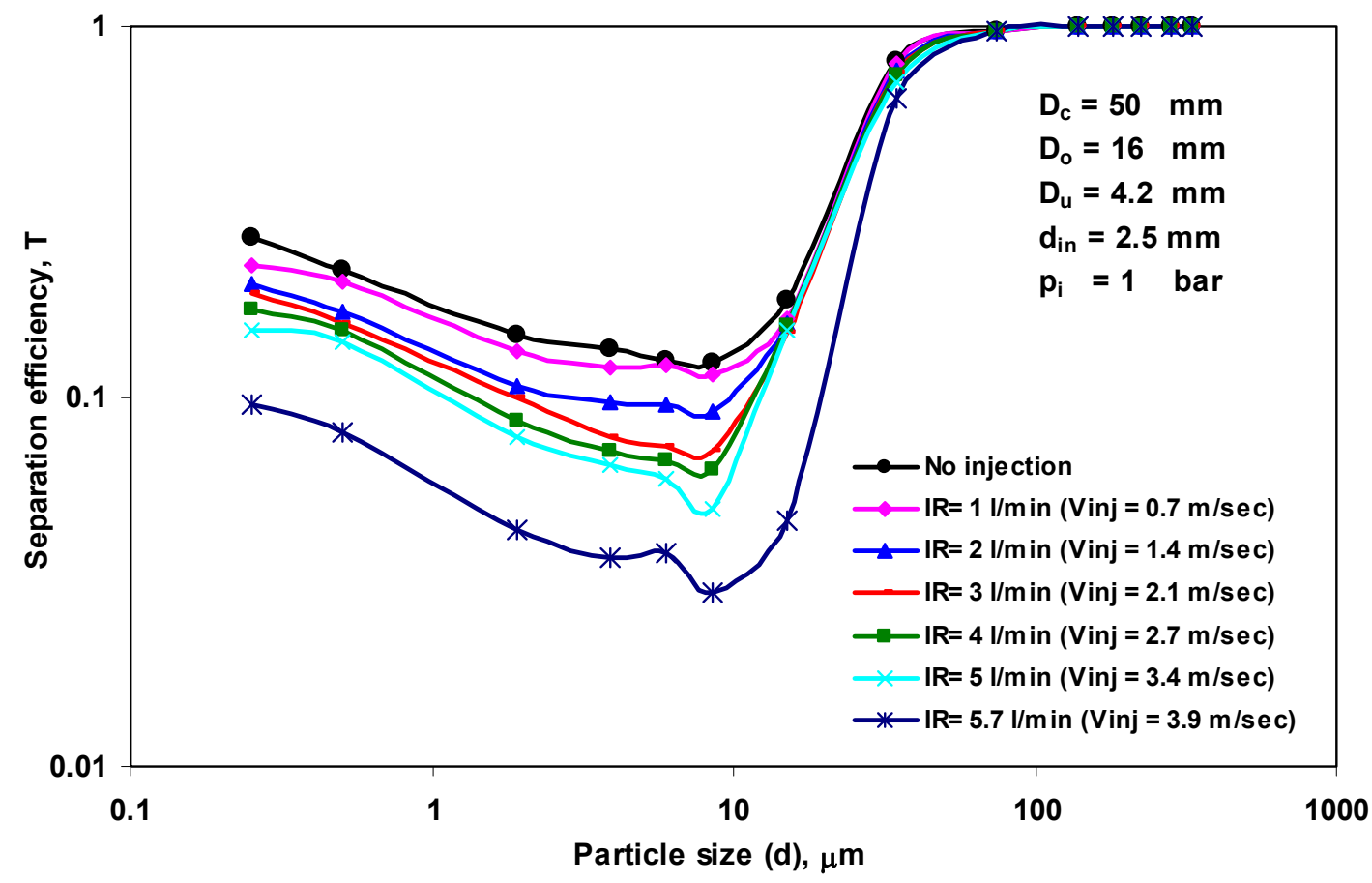

Fig. 7.40: Experimental separation curves at low injection velocities.

From Fig.7.40, it can be seen that the $T_{0}$ value decreases gradually while the cut size $\left(d_{50}\right)$ increases slightly with increasing the injection velocity (injection rate). This means that for 5 injection openings, no optimum injection velocity can be observed.

The results of the experiments carried out at low injection velocities (five injection openings) were simulated by Dueck et al. [10] using the Fluent 6.3 package to compare the experimental and the calculated data (see chapter 4). The computations of the separation curves were carried out by numerical solution of the Eqs. (4.2) (4.10). Five injection openings with injection diameter $d_{\text {in }}=2.5 \mathrm{~mm}$ were chosen for adjusting the injection velocity. The volume split parameter (S) was calculated from the experimental results and found out to be equal 9. Furthermore, the transport coefficients $V_{s, j}$ and the turbulent diffusion coefficient $D_{t}$ were determined with the mean tangential velocity $U_{\tan }[7]$ :

$$
U_{\tan }=3.7\left(\frac{D_{i}}{D_{c}}\right) U_{i}=5.8 \mathrm{~m} / \mathrm{s} .
$$


The turbulent diffusion coefficient [1] [59] is approximated using $D_{t}=U_{\tan }^{\prime} \lambda$, where $U_{\tan }^{\prime}$ is the fluctuation of the tangential velocity and $\lambda$ is the length scale of turbulence. Substituting the empirical relations $U_{\tan }^{\prime}=0.04 \mathrm{U}_{\tan }$ and $\lambda=0.05 D_{c}$, results in $D_{t}=1.16 \times 10^{-3} \mathrm{~m}^{2} / \mathrm{s}$.

The separation curves obtained from the numerical calculations are presented in Fig. 7.41. Figure 7.42 and Fig. 7.43 show the comparison between the experimental and the calculated data of the cut size $\left(\mathrm{d}_{50}\right)$ and the separation efficiency value of the fine particles $\left(\mathrm{T}_{0}\right)$ respectively.

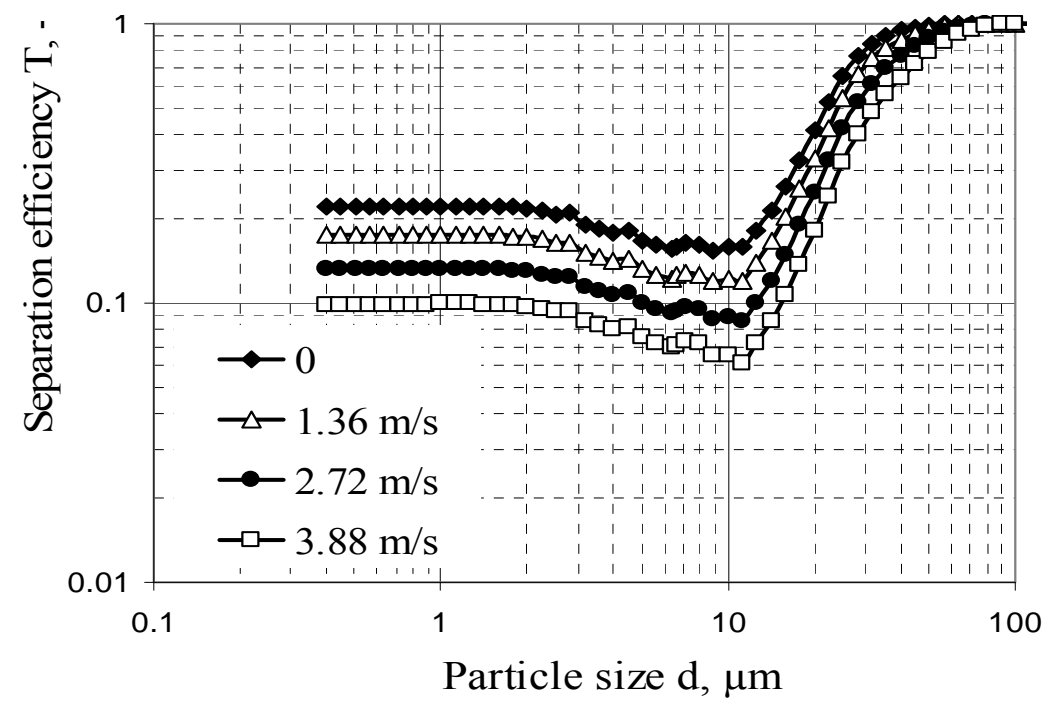

Fig. 7.41: Calculated separation curves for different low injection velocities.

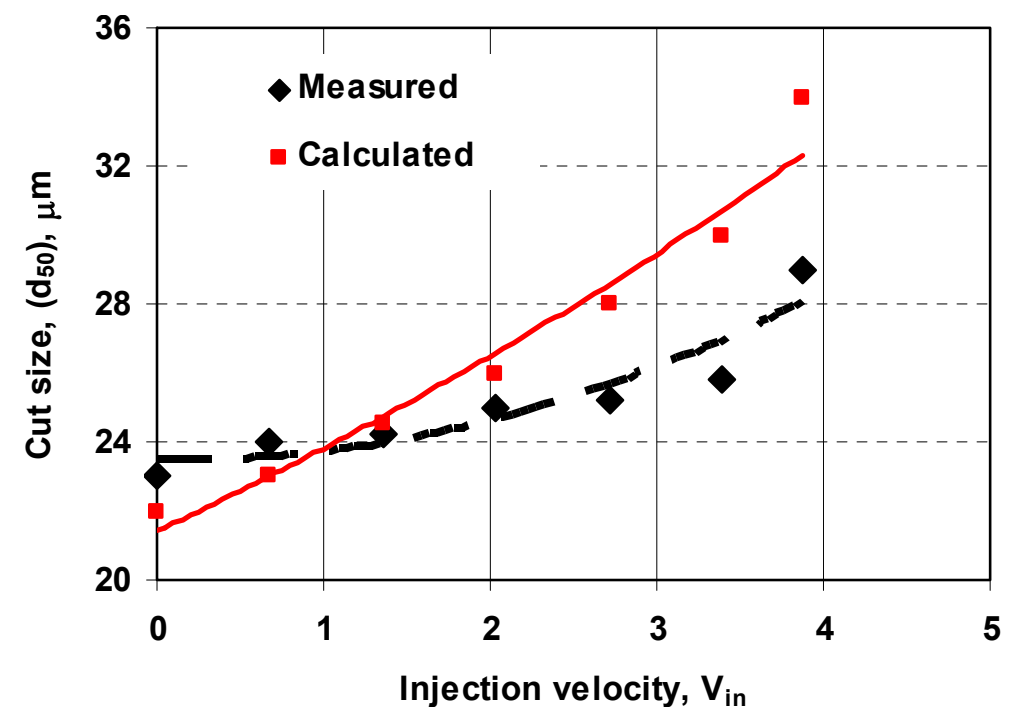

Fig. 7.42: Comparison of calculated and measured values of the cut size $\left(d_{50}\right)$ at different injection velocities. 


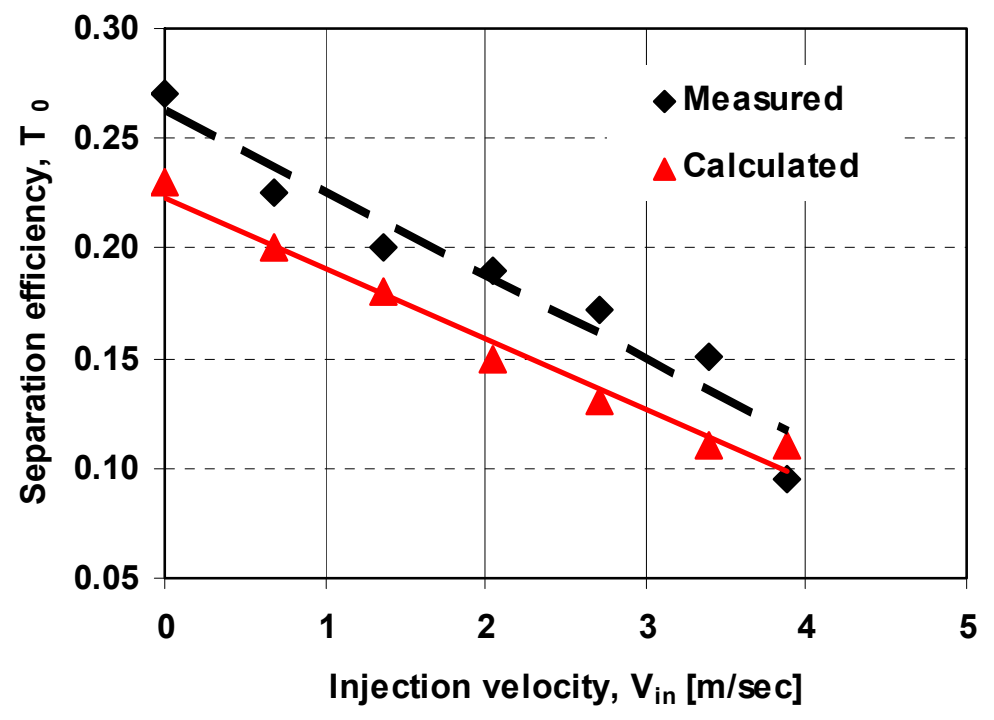

Fig. 7.43: Comparison of calculated and measured values of $T_{0}$ at different injection velocities.

These Figures show that the calculated separation curves are comparable with the measured ones with some deviations. Increasing the injection rate resulted in an increase in the cut size in the model than in the experiments. This may be due to the fact that the model does not account for the dilution effect caused by the injection, which improves the settling of the coarse particles as shown in Eq. (4.18). In addition, the "fish-hook" effect is less obvious in the model than in the experiments. This can be clearly seen in Fig.7.44 where the injection removes only the fine sizes from the underflow while the overflow quality does not change. Both the calculated and the experiments data showed the same trend. However, the $T_{0}$ values versus the $V_{\text {in, } 0}$ decrease approximately by the same rate for both cases. 


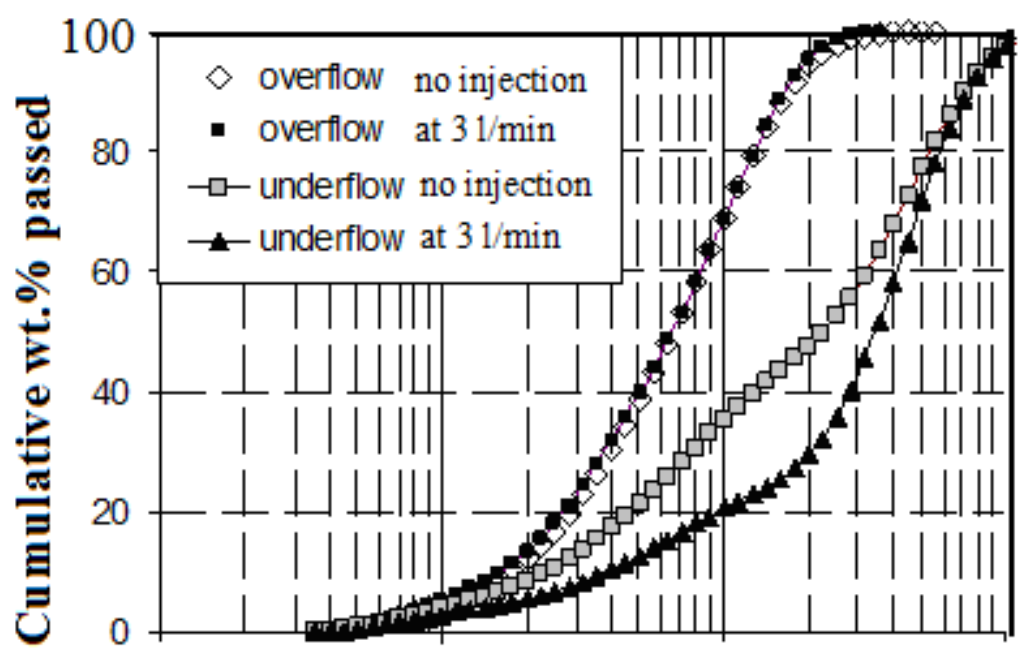

a)

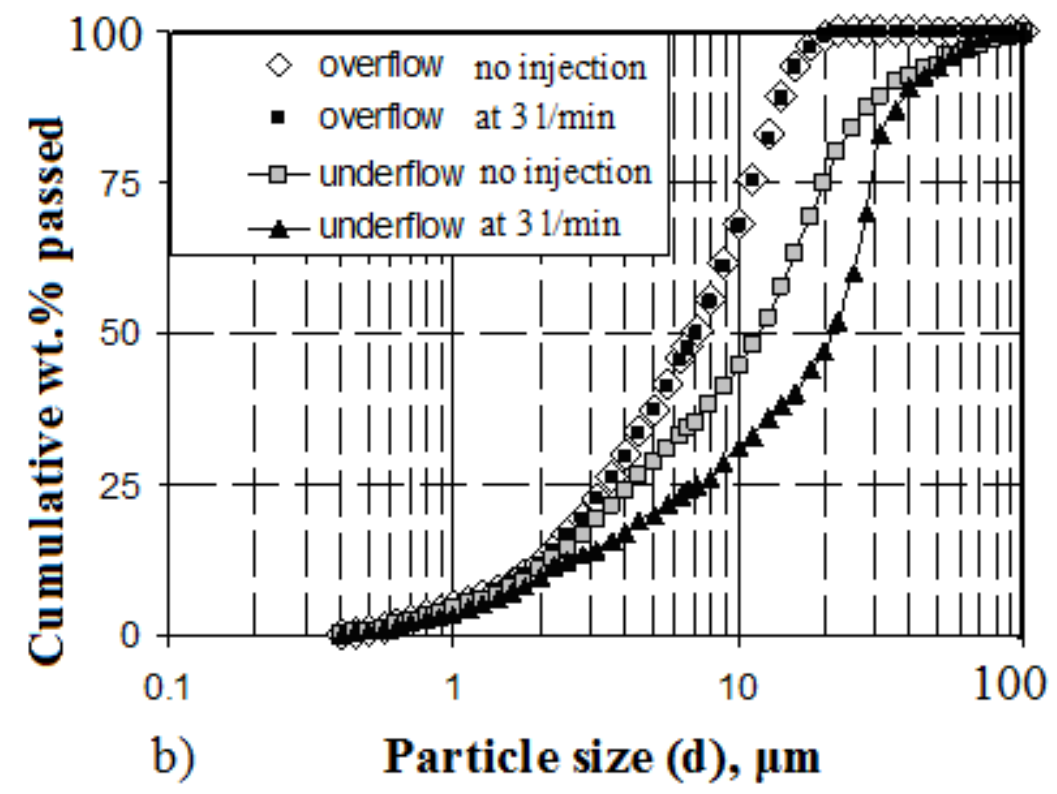

Fig. 7.44: Comparison of the calculated and measured particle size distributions of overflow and underflow without and with injection: a) - measurements, b) calculation.

Figure 7.45 shows a comparison between the experimental and calculated values of the underflow solid recovery. It was found that the increase in the injection velocity decreased the solid recovery gradually in the underflow with the same rate in both the experimental and calculated data. This should be due to the injection effect where more fines leave the underflow and go to the overflow. 


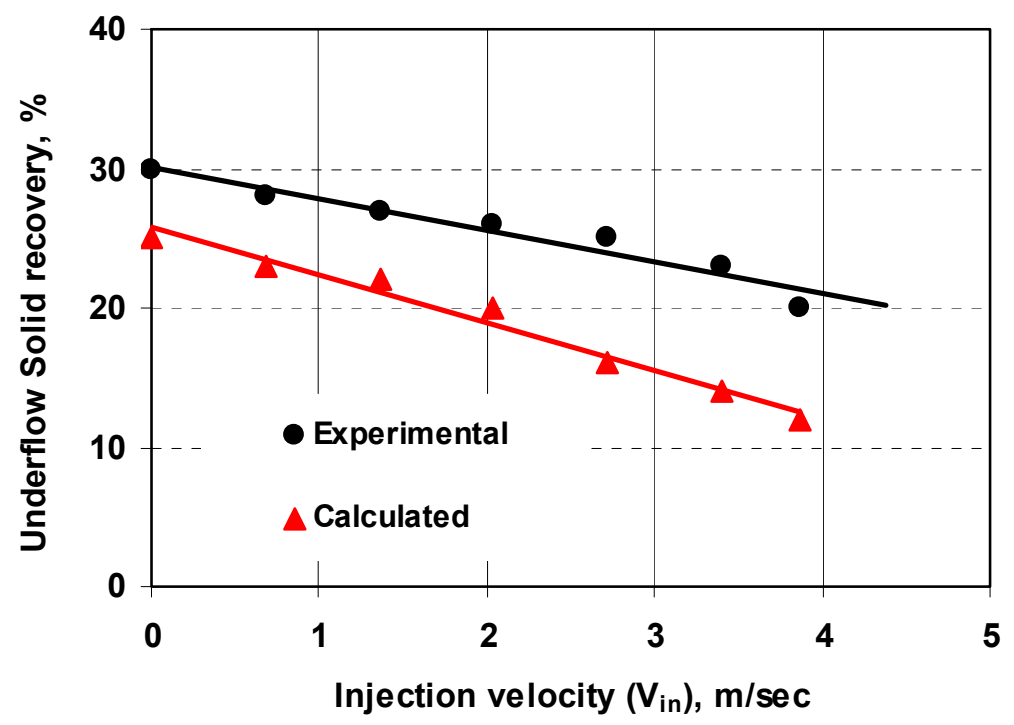

Fig.7.45: Comparison of calculated and measured underflow solid recovery at different injection velocities.

It can be generally concluded that both the calculated and the experimental data do agree in the trend with some deviations in the values.

\subsubsection{Effect of High Injection Velocities}

It was previously shown that the injection effect using three injection openings has the advantage of higher injection velocity than the five injection openings for the same injection rate. Therefore, all the parameters which influence the injection were investigated using three injection openings. Figure 7.46 shows the separation curves obtained at high injection velocities ranging from $3.15 \mathrm{~m} / \mathrm{sec}(1 \mathrm{l} / \mathrm{min})$ to $15.8 \mathrm{~m} / \mathrm{sec}$ (5 1/min). Figure 7.47 shows the separation efficiency values obtained at different injection rates (injection velocities). 


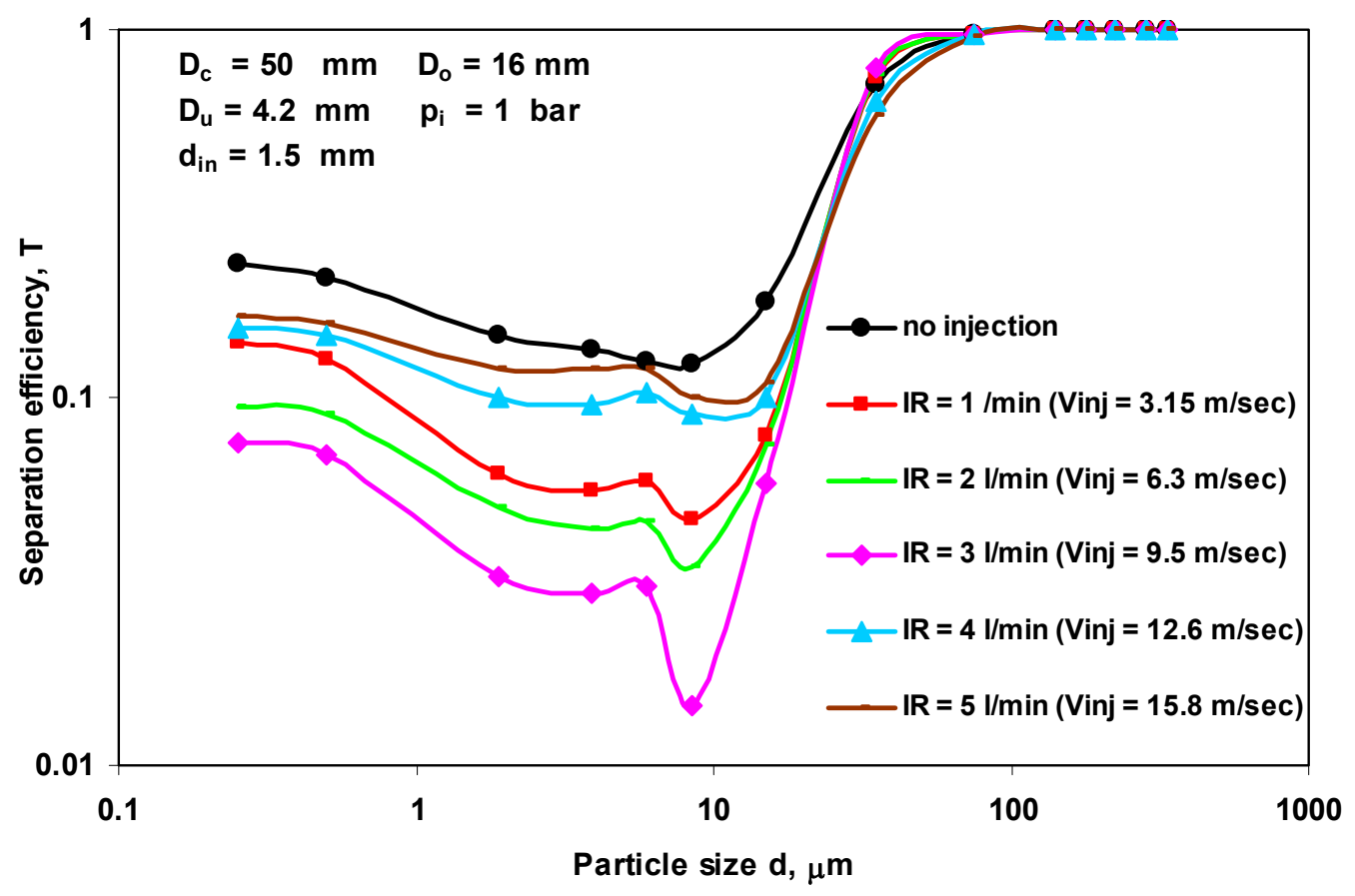

Fig. 7.46: Different separation curves obtained at high injection velocities.

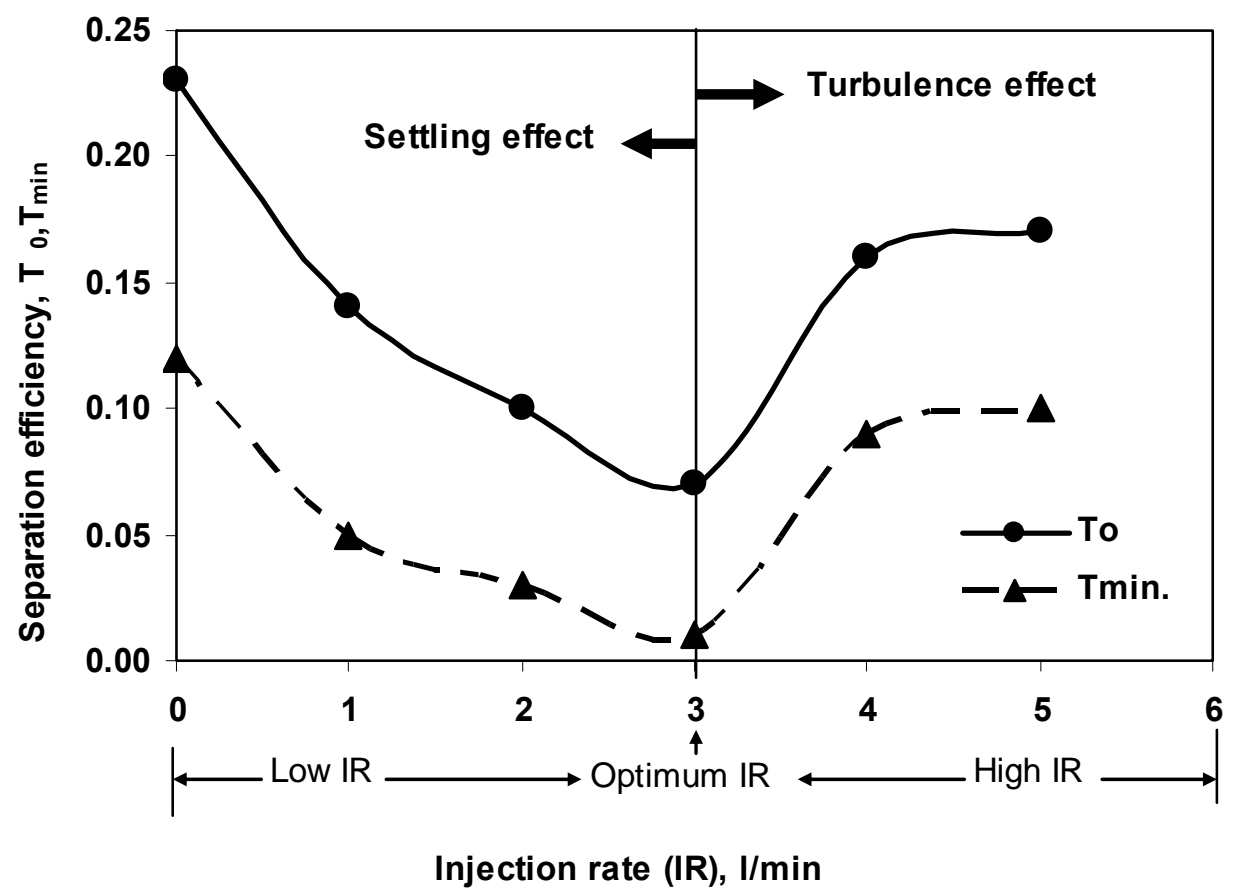

Fig. 7.47: Effect of injection rate on the separation efficiency of the fine particles $\left(\mathrm{T}_{0}\right.$, $\left.\mathrm{T}_{\min }\right)$. 
Figure 7.46 and Fig. 7.47 show that at low injection rates up to $31 / \mathrm{min}(9.5 \mathrm{~m} / \mathrm{sec})$, the $T_{0}$ value decreased gradually with increasing the injection rate. Further increase in the injection rate to $51 / \mathrm{min}(15.8 \mathrm{~m} / \mathrm{sec})$ increased the $T_{0}$ markedly.

The decrease in the $T_{0}$ value at low injection rates can be explained by Eq. (4.16), which shows that at low injection velocity, the improved settling conditions (negative settling velocity values) are determined. Then the radial directed injection flow transports fine particles to the centre of the cyclone and finally to the overflow.

At higher injection velocities another effect becomes important, which leads to a deterioration of the washing effect, i.e., an increase in the $T_{0}$ values versus $V_{\text {in }}$ as can be seen from Fig. 7.46. A possible explanation for this effect, based on the separation function Eq. (4.15), can be given as follows:

It can be assumed that a stable diffusion coefficient exists at low injection rates. The diffusion coefficient $D_{t}\left(V_{i n, 0}\right)$ increases if the radial component of the injection velocity $V_{i n}, 0$ exceeds a critical value which can be approximated empirically by:

$D_{t}\left(V_{i n, 0}\right)=D_{t}^{0}\left(1+\beta V_{i n, 0}^{\gamma}\right)$, where, $\gamma \geq 1$.

Substituting Equation (7.10) in Equation (4.15), another expression for $T_{0}$, different from that in Eq. (4.16), is obtained as follows:

$$
T(0)=\frac{1}{1+S \exp \left[\frac{h}{D_{t}^{0} \beta V_{i n, 0}^{\gamma-1}}\right]} .
$$

It can be seen that Eq. (7.11) gives higher $T_{0}$ values than Eq. (4.16) which means that at high injection velocity a large fraction of the fine particles are misplaced in the underflow. This explains well the increase in the $T_{0}$ values at higher injection velocities (higher than the optimum velocity). Accordingly, the effect of the injection on the fine particles separation in the underflow can be summarized as a combination of two phenomena. First, the counter current (settling) effect at low injection rates dominates until an optimum injection rate is reached. Then, at higher 
injection rates, the effect of high turbulence diffusion dominates, and reduces the injection effect.

As can be shown in Fig. 7.48, the simulation closely follows the experimental values only in the range of low injection velocity up to $4 \mathrm{~m} / \mathrm{sec}$ (the red curves). Further increase in the injection velocity to $9.5 \mathrm{~m} / \mathrm{sec}$ (the optimum injection velocity) increases the washing effect when the counter current effect of the injection dominants as shown in Fig. 7.47. Fig. 7.48 shows also that the optimum injection velocity is higher than the feed tangential velocity $(6.5 \mathrm{~m} / \mathrm{sec})$. This is considered the basic precondition for achieving an optimum washing effect. More increase in the injection velocity to $16 \mathrm{~m} / \mathrm{sec}$, more turbulence is produced which disrupts the separation. This influence can be accounted for in a different model operating by using an increased turbulent diffusion coefficient.

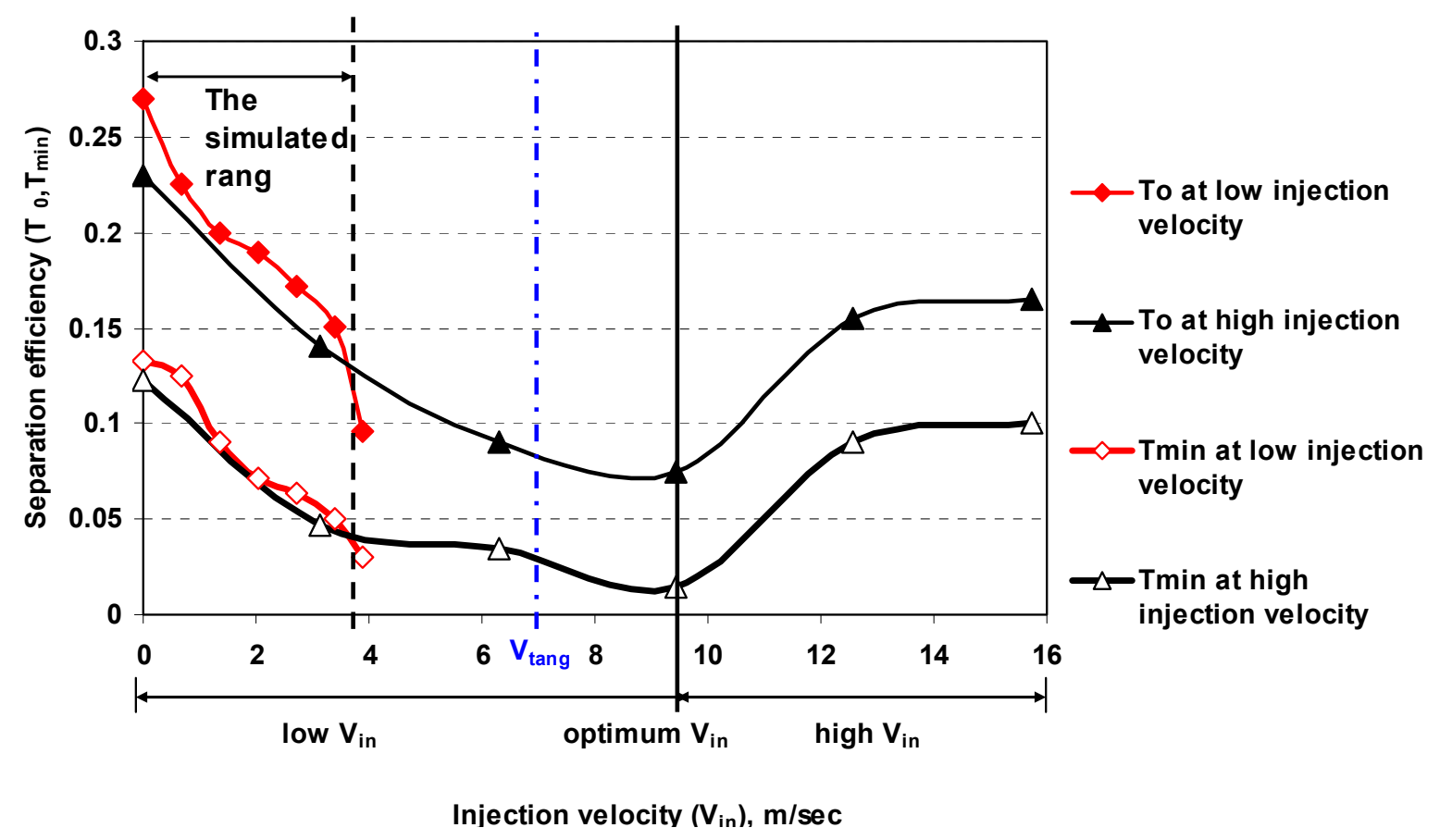

Fig. 7.48: Calculated and experimental values of $T_{0}$ and $T_{\min }$ at different injection velocities.

\subsubsection{Effect of Injection Rate (IR) on the Cut Size $\left(d_{50}\right)$ and Imperfection (I)}

Fig. 7.49 shows that at low injection rates up to $31 / \mathrm{min}(9.5 \mathrm{~m} / \mathrm{sec})$, the cut size $\left(\mathrm{d}_{50}\right)$ is almost stable or slightly decreases. 


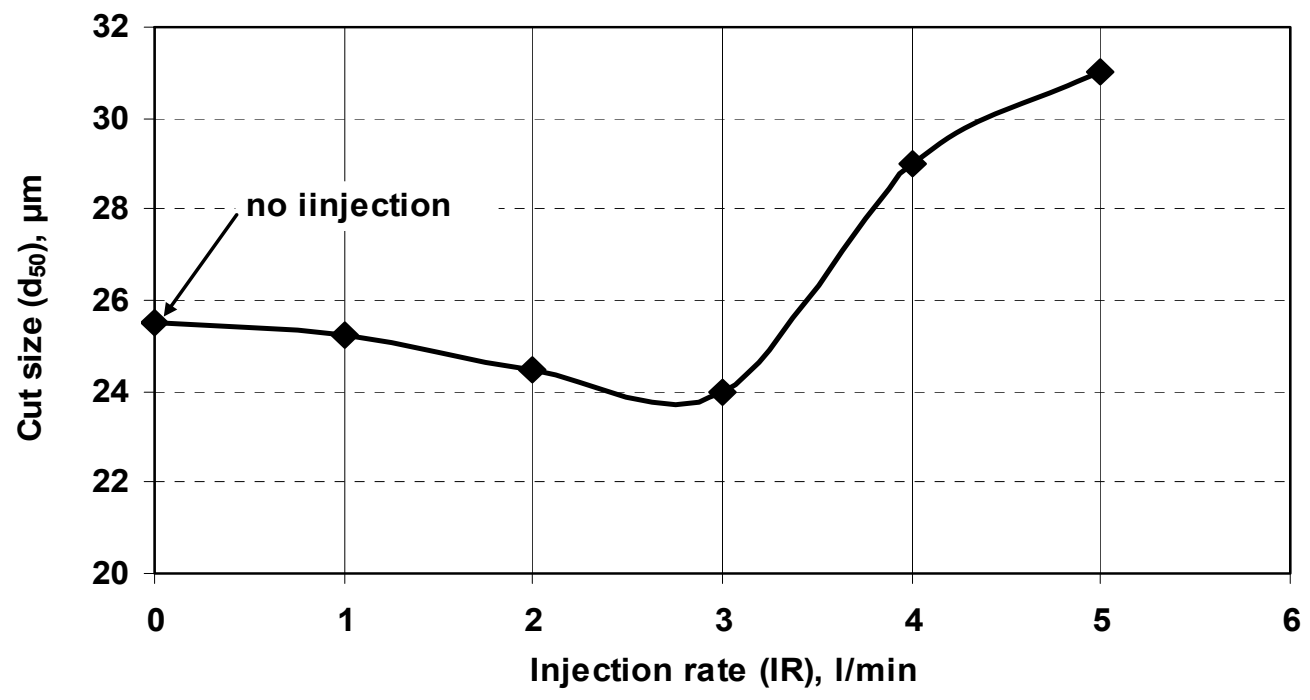

Fig. 7.49: Effect of injection rate on the cut size $\left(\mathrm{d}_{50}\right)$.

This may be due to the influence of the optimum injection rate which affects only the fines and does not cause more turbulence as it was presented in by Fig. 7.48. This can also be explained by the dilution effect which improves the settling conditions of the coarse particles as it was shown in Eq. (4.18).

Moreover, as it was previously shown in Fig. 7.44, the particle size analysis of the overflow and underflow indicated that the water injection only changes the distribution of the underflow sharply (reducing only the fine fractions percent) while, nearly no change occurred in the overflow size distribution. Therefore, a constant cut size $\left(d_{50}\right)$ should be expected at the optimum injection rate $(3 \mathrm{l} / \mathrm{min})$. Such results are important in practice because until now, all the reviewed hydrocyclone literature which use the water injection technique are still suffering from the disadvantage of increasing the cut size $\left(\mathrm{d}_{50}\right)$ which accompanies the injection effect.

Figure 7.48 shows also that by further increase in the injection rate higher than $31 / \mathrm{min}$ and up to $5 \mathrm{l} / \mathrm{min}$ the cut size $\left(\mathrm{d}_{50}\right)$ increased from $25 \mu \mathrm{m}$ to $31 \mu \mathrm{m}$. This may be due to the high disturbance in the turbulence diffusion which is caused by the high injection rates. Another explanation of the increase of the cut size at high injection rates can be given as follows. In the case of the high injection rates, the injection flow could overcome the settling rate of the coarse particles to the wall, and then carry them through it and across the locus of the zero axial velocity towards the hydrocyclone centre, and in turn, to the overflow. This may result in higher cut sizes. 
Figure 7.50 shows the effect of the injection rate on the imperfection. It shows that at low injection rates, the imperfection decreases down to an optimum value at $3 \mathrm{l} / \mathrm{min}$ $(9.5 \mathrm{~m} / \mathrm{sec})$. This may be due to the dilution effect which creates better settling conditions for the coarse particles and improves the separation. By increasing the injection rate up to $5 \mathrm{l} / \mathrm{min}(16 \mathrm{~m} / \mathrm{sec})$, the imperfection value increases markedly. This may be due to the increase of the turbulent diffusion which disturbs the separation.

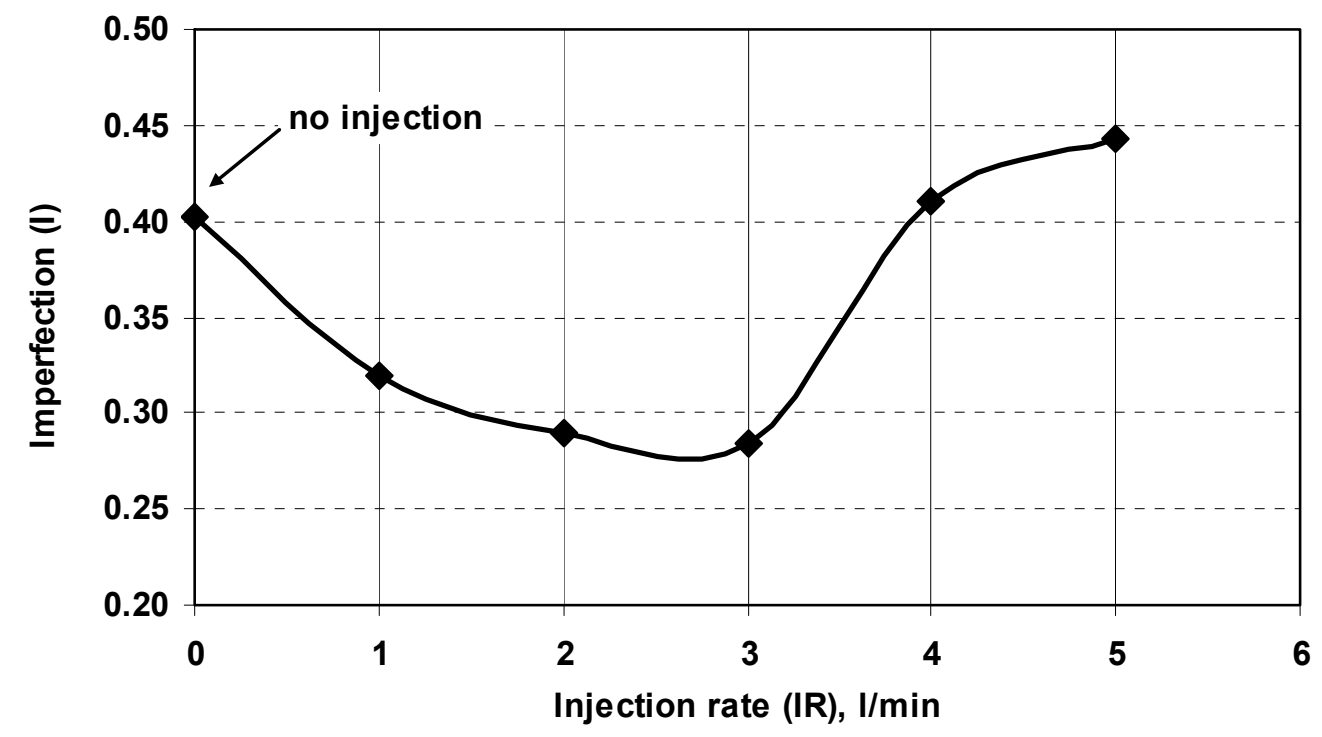

Fig. 7.50: Effect of injection rate on the imperfection.

\subsubsection{Effect of Injection Rate (IR) on the Solid Recovery and Solid Content.}

Figure 7.51 shows the solid recovery in the underflow at different injection rate $(1 / \mathrm{m})$.

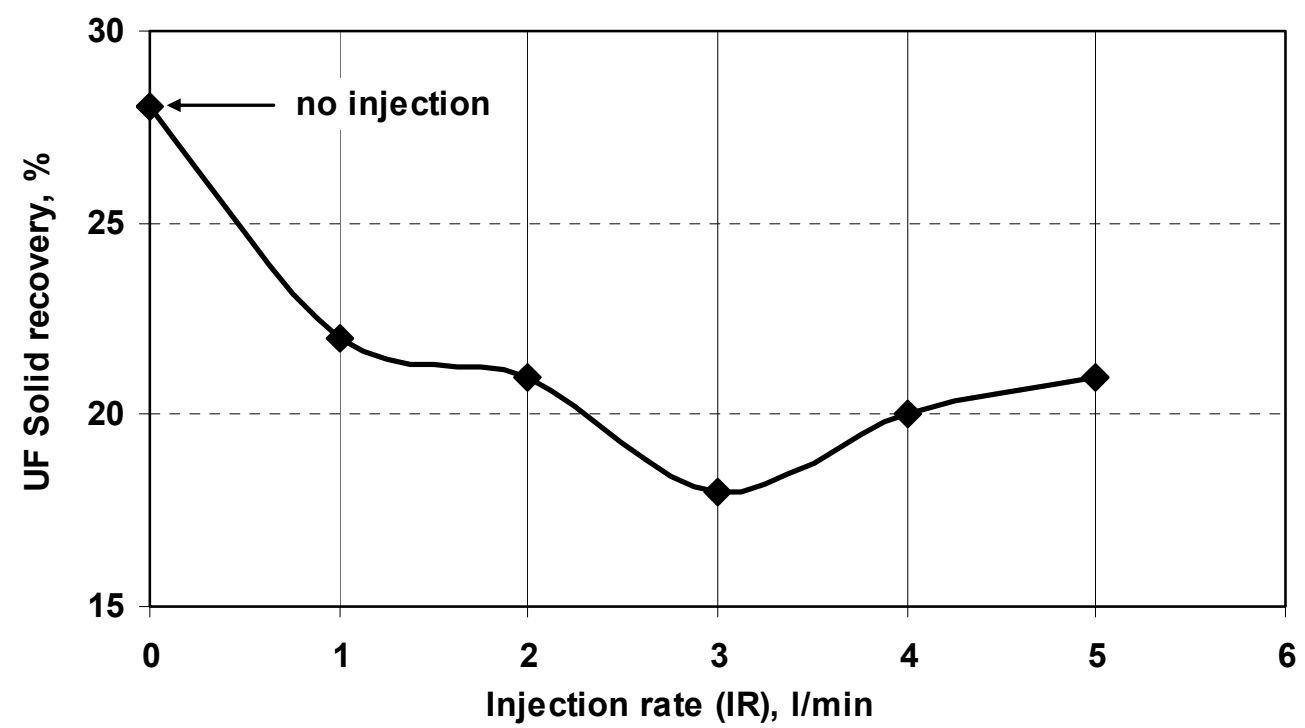

Fig. 7.51: Effect of injection rate on the underflow solid recovery. 
As can be seen from Fig. 7.51, at low injection rates, the solid recovery decreased with increasing the injection rate. This may be due to the removal of fines from the underflow to the overflow by the injection effect. At high injection rates, the solid recovery increased. This may be due to the dilution effect which leads to an increase in the discharge of the fines to the underflow which results in a marked decrease in the underflow solid content as it can be seen from Fig. 7.52.

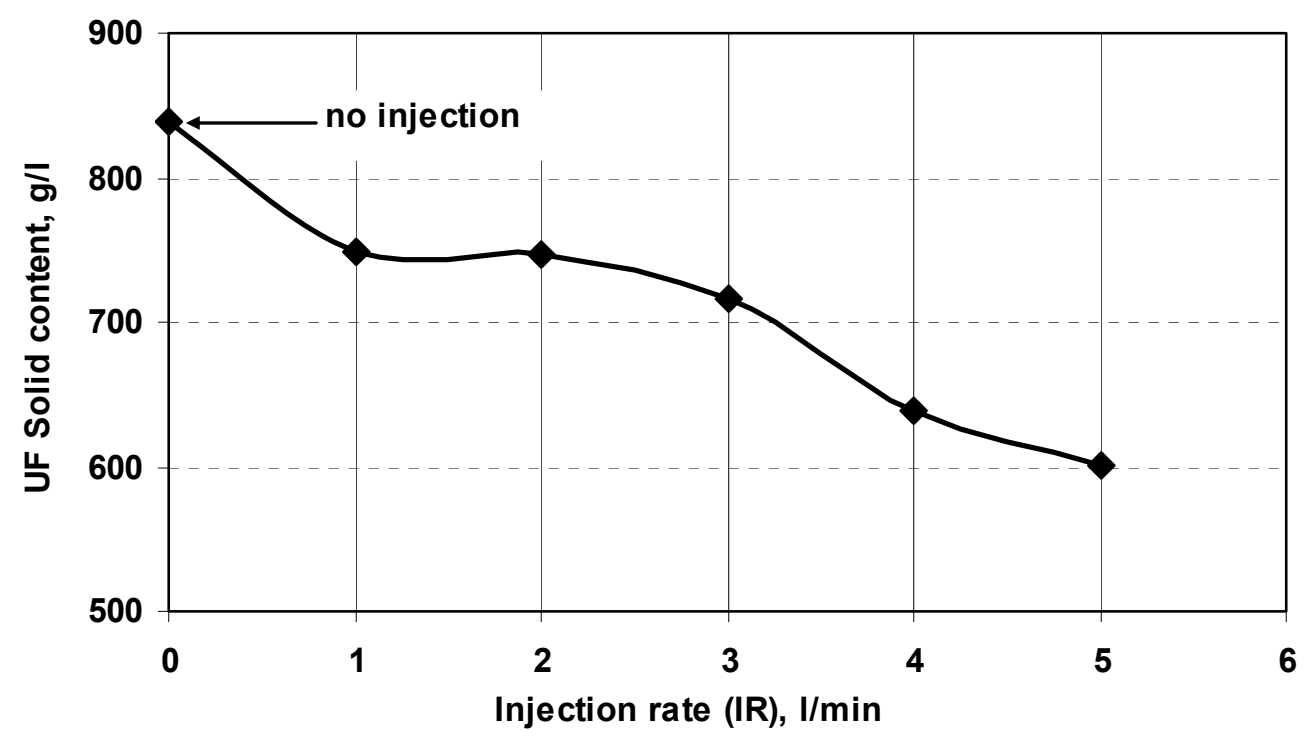

Fig. 7.52: Effect of injection rate on the underflow solid content $(\mathrm{g} / \mathrm{l})$.

The results indicate that there is an optimum injection rate of about $3 \mathrm{l} / \mathrm{min}(9.5$ $\mathrm{m} / \mathrm{sec}$ ) in the case of using three injection openings. At this injection rate an optimum effect was achieved for all the investigated relations.

\subsubsection{Effect of Underflow Diameter $\left(D_{u}\right)$}

\subsubsection{Effect of Underflow Diameter on the Separation Efficiency of the Fine Particles$$
\left(\mathbf{T}_{\mathbf{0}}, \mathbf{T}_{\mathrm{min}}\right)
$$

The underflow opening diameter is an important parameter in the hydrocyclone practice because it influences the solids discharge. It is considered the major parameter which can be used to manipulate the separation performance easily. In the present work, it was important to start with the available small underflow diameter 
which gives a thick suspension (rope discharge) to reduce the feed water in the underflow. Accordingly, the less the water in the underflow, the smaller is the injected water injection required to displace it, and the higher is the injection effect.

Figure 7.53 and Fig. 4.54 show the effect of changing the underflow diameter on the separation efficiency values.

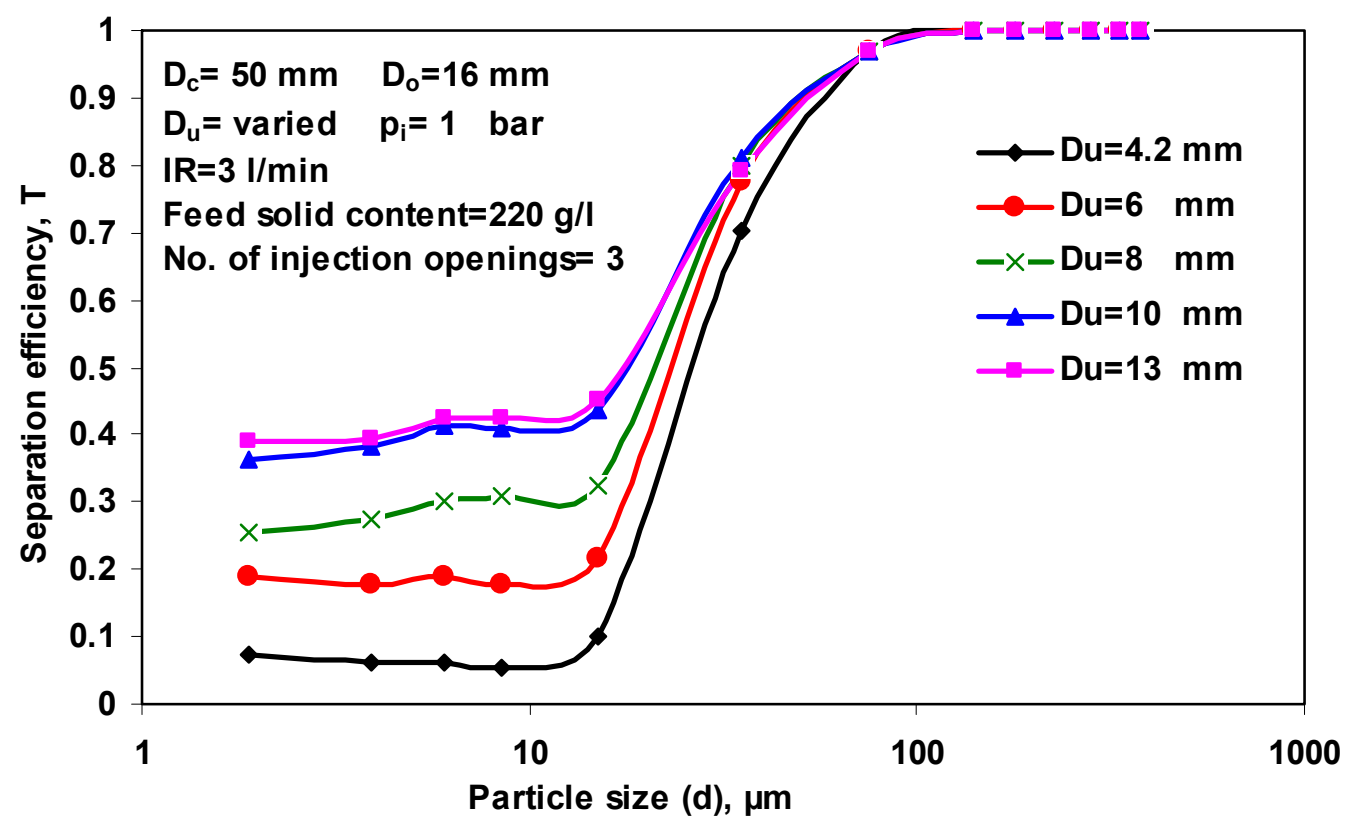

Fig. 7.53: Different separation curves obtained at different underflow diameters $\left(D_{u}\right)$.

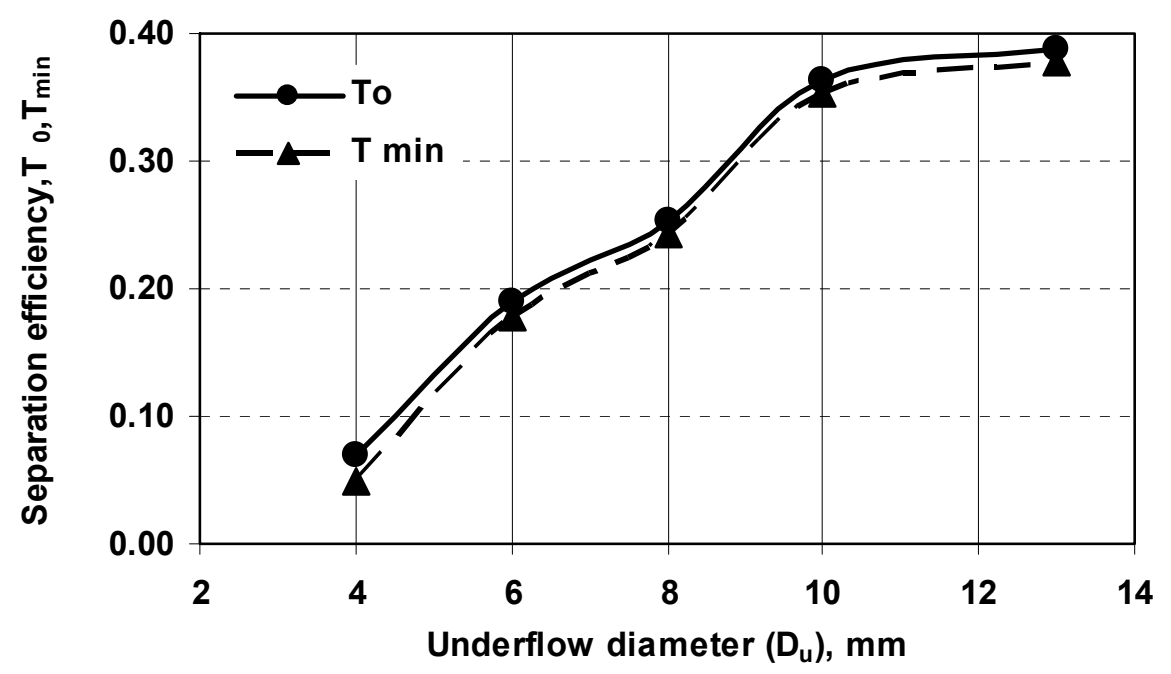

Fig. 7.54: Effect of the underflow diameter on the separation efficiency of the fine particles $\left(\mathrm{T}_{0}, \mathrm{~T}_{\min }\right)$. 
From Fig. 7.54, it can be shown that the $T_{0}$ and $T_{\min }$ values increase with increasing the underflow diameter. This can be explained based on the formula of the separation curve given by Schubert and Neesse [54]. This formula can be expressed as follows:

$$
T_{\min }=\frac{1}{1+\frac{Q_{O F}}{Q_{U F}}},
$$

where, $Q_{O F}$ and $Q_{U F}$ are the water flow rates in the overflow and underflow respectively in the same units. According to Tarjan [97], the ratio of the overflow volume flow rate and the underflow flow rate is proportionally related to the overflow and underflow diameters as follows:

$$
\frac{Q_{O F}}{Q_{U F}} \approx 0.91 *\left(\frac{D_{o}}{D_{u}}\right)^{3} .
$$

Plitt [101] has estimated the same relation in another form as follows.

$$
\frac{Q_{O F}}{Q_{U F}} \approx\left(\frac{D_{o}}{D_{u}}\right)^{3.4} .
$$

From Eq. (7.12) and Eq. (7.13), Eq. (7.11) can be rewritten as follows:

$$
T_{\min }=\frac{1}{1+\left(\frac{D_{o}}{D_{u}}\right)^{3}} .
$$

From Eq. (7.15), it can be clearly shown that the separation efficiency value of the fine particles $T_{\min }$ increases with increasing the underflow diameter.

A further reason for the increase in the fines percent at high underflow diameters is the increase in the discharge capacity of the apex which results in more feed water going to the underflow with more fines. The relation between the apex diameter $\left(D_{u}\right)$ 
and its solids capacity $\left(M_{u s}\right)$ was investigated by many researchers [61], [103], [105]. They gave the same formula with different values of the exponent $m$ as follows:

$M_{u s}=0.43 D_{u}^{m}$,

where, $m$ ranging between 2.12 and 2.35 .

\subsubsection{Effect of Underflow Diameter on the Cut Size $\left(d_{50}\right)$ and Imperfection (I).}

Figure 7.55 shows that the cut size $\left(d_{50}\right)$ decreases gradually with increasing the underflow diameter $D_{u}$. This can be explained by Eq. (3.3) for Schubert and Neesse [1] where the cut size $\left(d_{50}\right)$ is inversely proportional to the underflow diameter $\left(D_{u}\right)$.

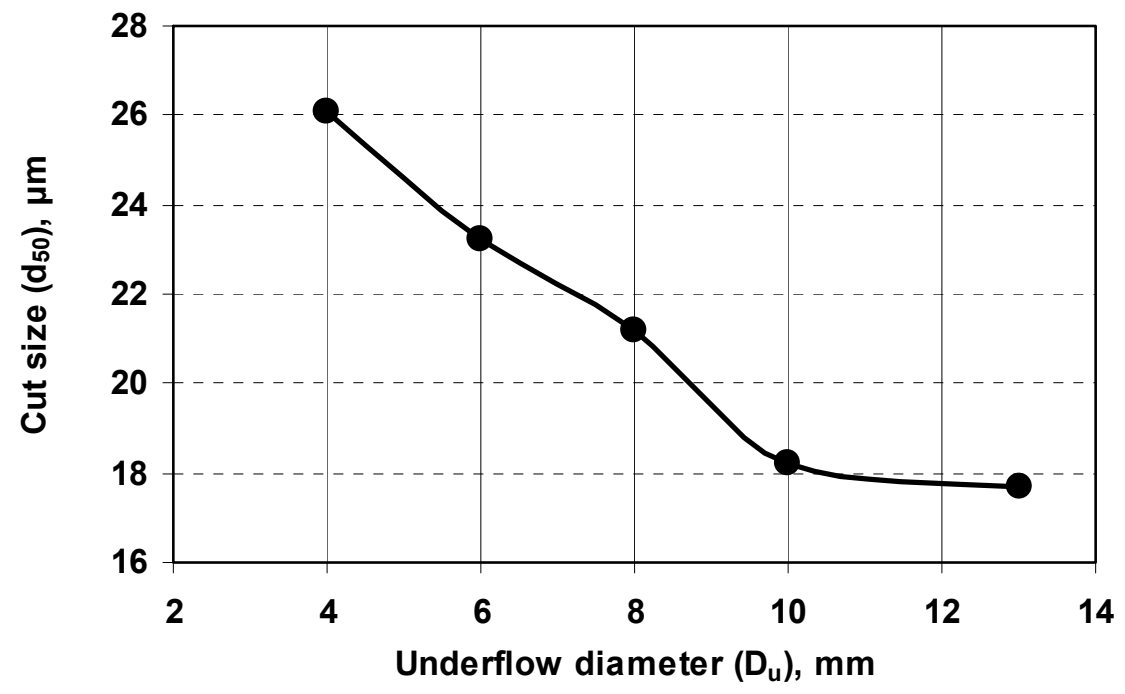

Fig. 7.55: Effect of the underflow diameter on the cut size $\left(\mathrm{d}_{50}\right)$.

Plitt has also put down a relation between the underflow diameter and the cut size $\left(\mathrm{d}_{50}\right)$ as follows;

$d_{50}=f\left(D_{u}^{-t}\right)$,

where, $t$ is about 0.71 . This relation was reported by many researchers [7] in the same form but with different values of the variable $a$ ranging between 0.5 and 0.9 . Mizrahi and Cohen [106] concluded that the cut size $\left(d_{50}\right)$ is changed when the cyclone starts 
roping instead of spray discharge. This result agrees with the literature reviewed concerning the injection effect.

Fig. 7.56 shows that the imperfection value increases gradually by increasing the underflow diameter. This may be a normal reflection of the increase in the cut size $\left(\mathrm{d}_{50}\right)$ according to Eq. (3.5).

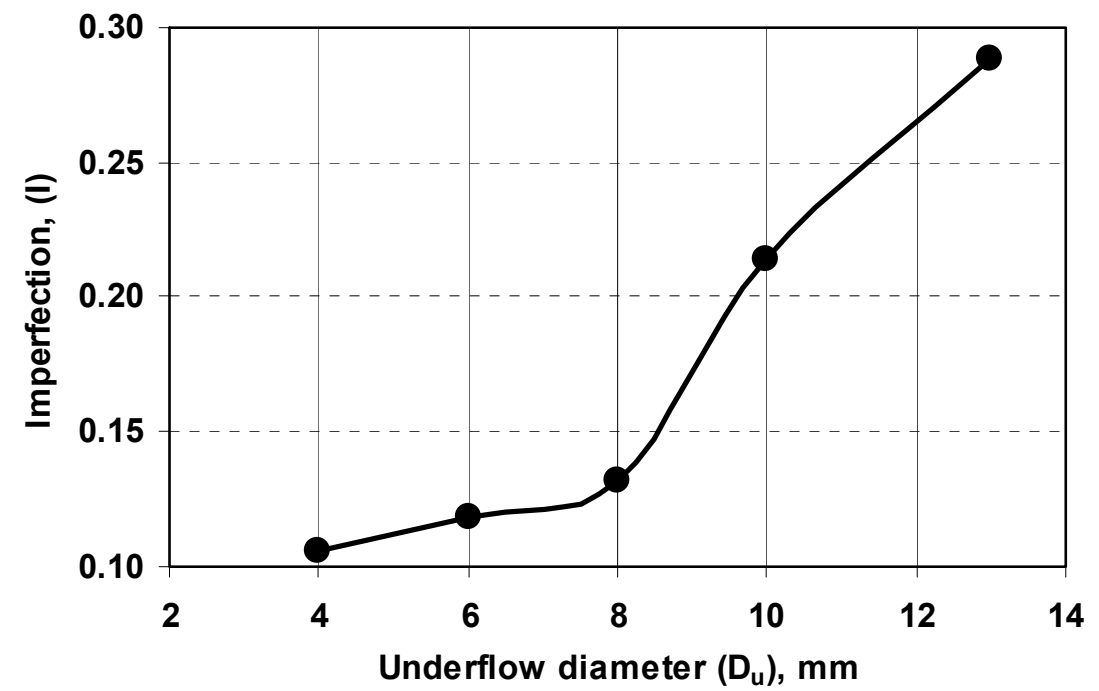

Fig. 7.56: Effect of the underflow diameter on the imperfection (I).

\subsubsection{Effect of Underflow Diameter on the Solid Recovery and Solid Content}

Figure 7.57 shows that the underflow solid recovery increases sharply with increasing the underflow diameter. This may be due to the increase in the discharge capacity of the apex as it was previously shown by Eq. (7.16).

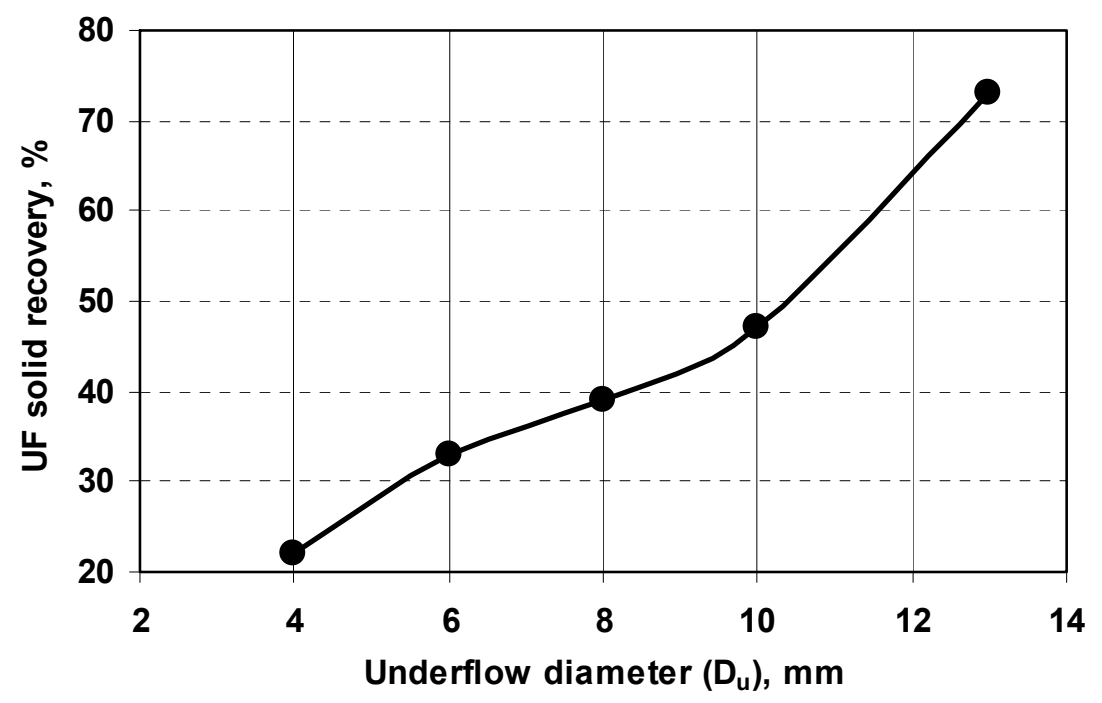

Fig. 7.57: Effect of the underflow diameter on the underflow solid recovery. 
As can be seen from Fig. 7.58 the underflow solid content decreases sharply by increasing the underflow diameter.

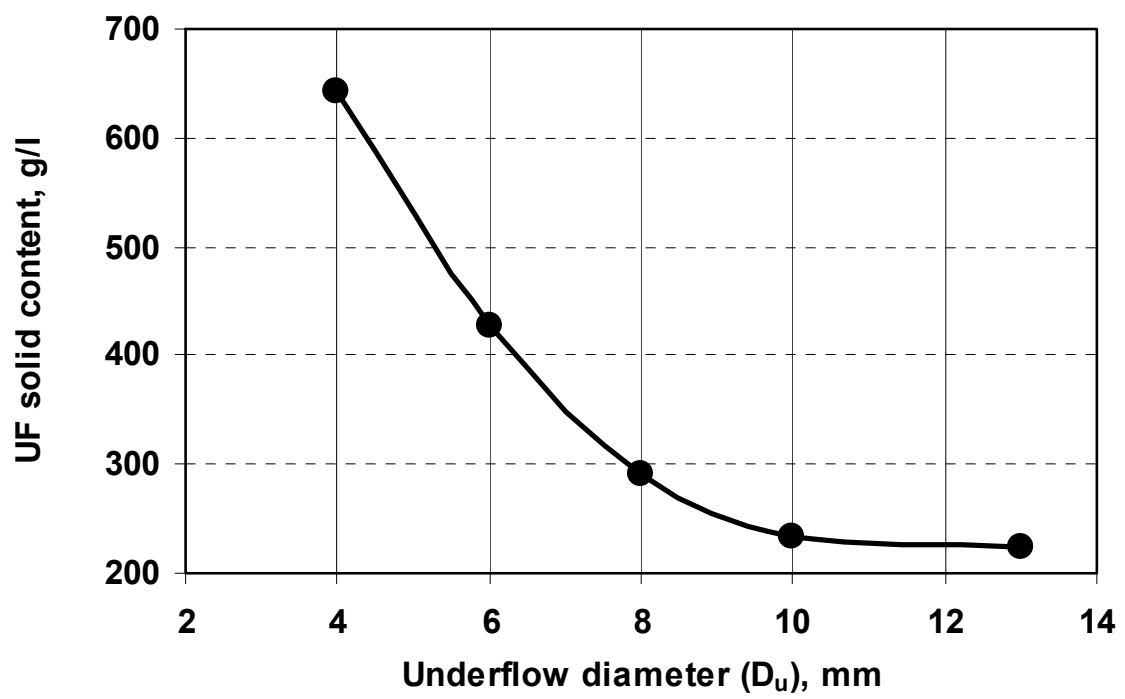

Fig. 7.58: Effect of the underflow diameter on the underflow solid content $(\mathrm{g} / \mathrm{l})$.

This may be due to the high dilution effect at high underflow diameter. This dilution reduces the suspension viscosity which leads to a higher suspension recovery. This will result in more water in the underflow and lower solid content. Therefore, it was important to carry out the experiments of the present work using the possible small apex to start with underflow rope discharge without injection.

\subsubsection{Effect of Overflow Diameter $\left(D_{o}\right)$}

The overflow diameter is considered the decisive parameter which determines the split parameter $\left(D_{0} / D_{u}\right)$. Therefore, an increase in the overflow diameter should have the same effect of a decrease in the underflow diameter. This is obviously valid only in the case without injection. In the case of injection, the water is injected near the apex. Therefore, the conditions at the underflow become decisive and the influence of the overflow diameter is not so determining for the process.

\subsubsection{Effect of Overflow Diameter on the Separation Efficiency of the Fine Particles $\left(\mathbf{T}_{\mathbf{0}}, \mathbf{T}_{\min }\right)$}


Figure 7.59 and Fig. 7.60 show the separation curves and the separation efficiency of the fine particles obtained at different overflow diameter respectively.

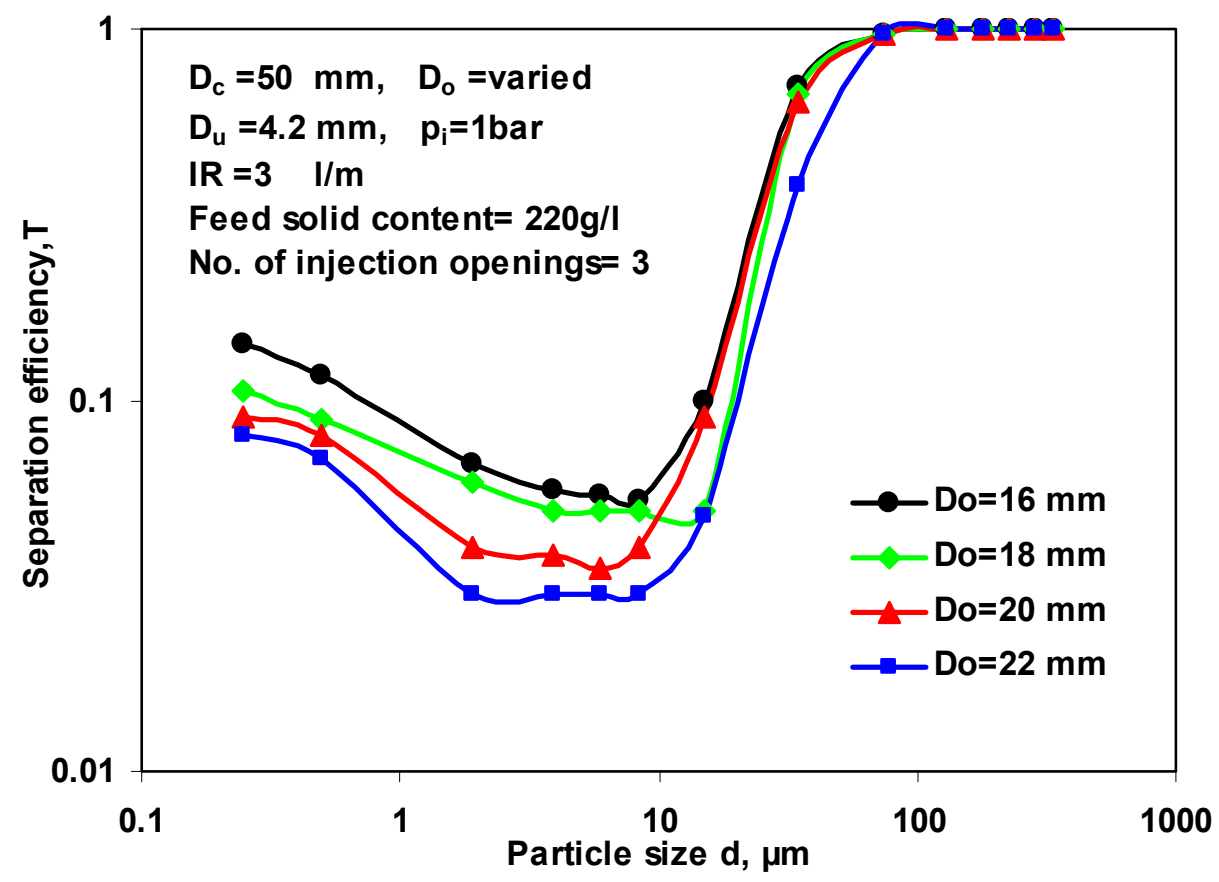

Fig. 7.59: Different separation curves at different overflow diameters $\left(D_{0}\right)$.

As can be seen from Fig. 6.59, there is no big difference between the separation curves of the different overflow diameter as it was previously discussed.

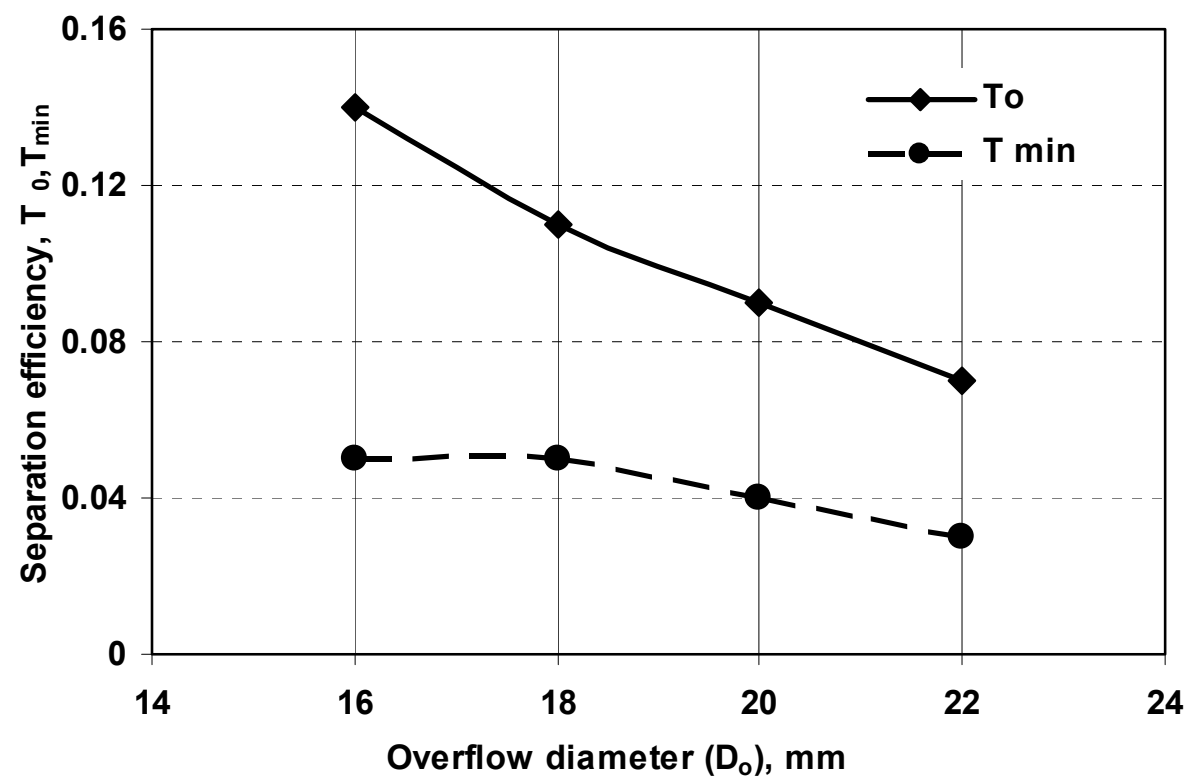

Fig. 7.60: Effect of the overflow diameter on the separation efficiency of the fine particles $\left(\mathrm{T}_{0}, \mathrm{~T}_{\min }\right)$. 
Figure 7.60 shows that the $T_{0}$ value decreases with increasing the overflow diameter. This can be explained according to Eq. (4.16) in which the $T_{0}$ value is inversely proportional to the split parameter $\left(S=D_{o} / D_{u}\right)$. Accordingly, the higher the overflow diameter; the lower is the $T_{0}$ value. This result agrees with the results obtained by Honaker et al. [87].

\subsubsection{Effect of Overflow Diameter on the Cut Size $\left(d_{50}\right)$ and Imperfection (I).}

Figure 7.61 and Fig. 6.62 show the effect of changing the overflow diameter on cut size $\left(\mathrm{d}_{50}\right)$ and the imperfection (I) respectively.

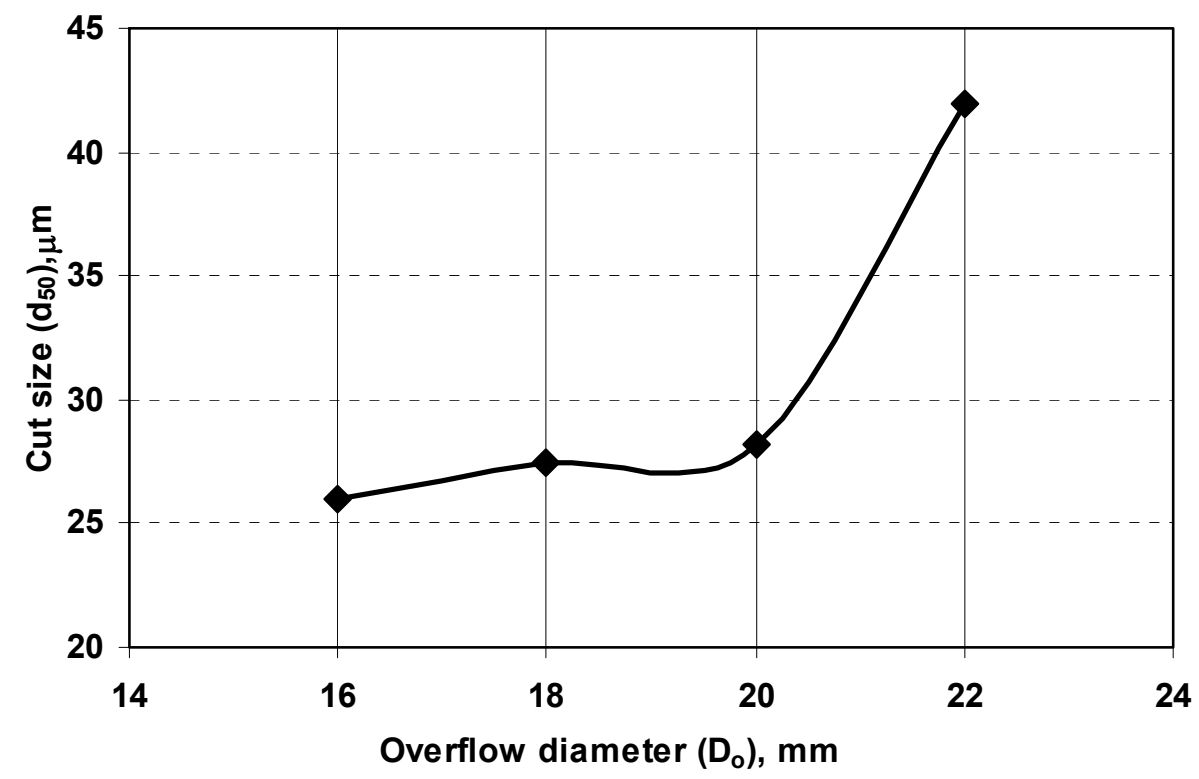

Fig. 7.61: Effect of overflow diameter on the cut size $\left(\mathrm{d}_{50}\right)$.

From Fig. 7.61, it can be shown that the cut size increases with increasing the overflow diameter. This can be explained by Eq. (3.3) of Schubert and Neesse [1] where the cut size $\left(d_{50}\right)$ is directly influenced by the overflow diameter $\left(D_{0}\right)$.

According to Plitt [98], the increase in the vortex finder diameter increases the cut size $\left(\mathrm{d}_{50}\right)$ according to the following empirical formula:

$d_{50}=f\left(D_{o}\right)^{1.21}$. 
Many researchers [107,108] have suggested the same form of Eq. (7.18) but with different exponent ranging from 0.8 up to 1.85 . Accordingly, it is clear that the cut size $\left(d_{50}\right)$ increases with increasing the overflow diameter $\left(D_{0}\right)$.

Fig. 7.62 shows that the Imperfection decreases with increasing the overflow diameter. This may be due to the increase in the cut size $\left(\mathrm{d}_{50}\right)$ which is inversely proportional to the imperfection value as shown in Eq. (1.2). This result agrees with the work of Udaya et al. [89].

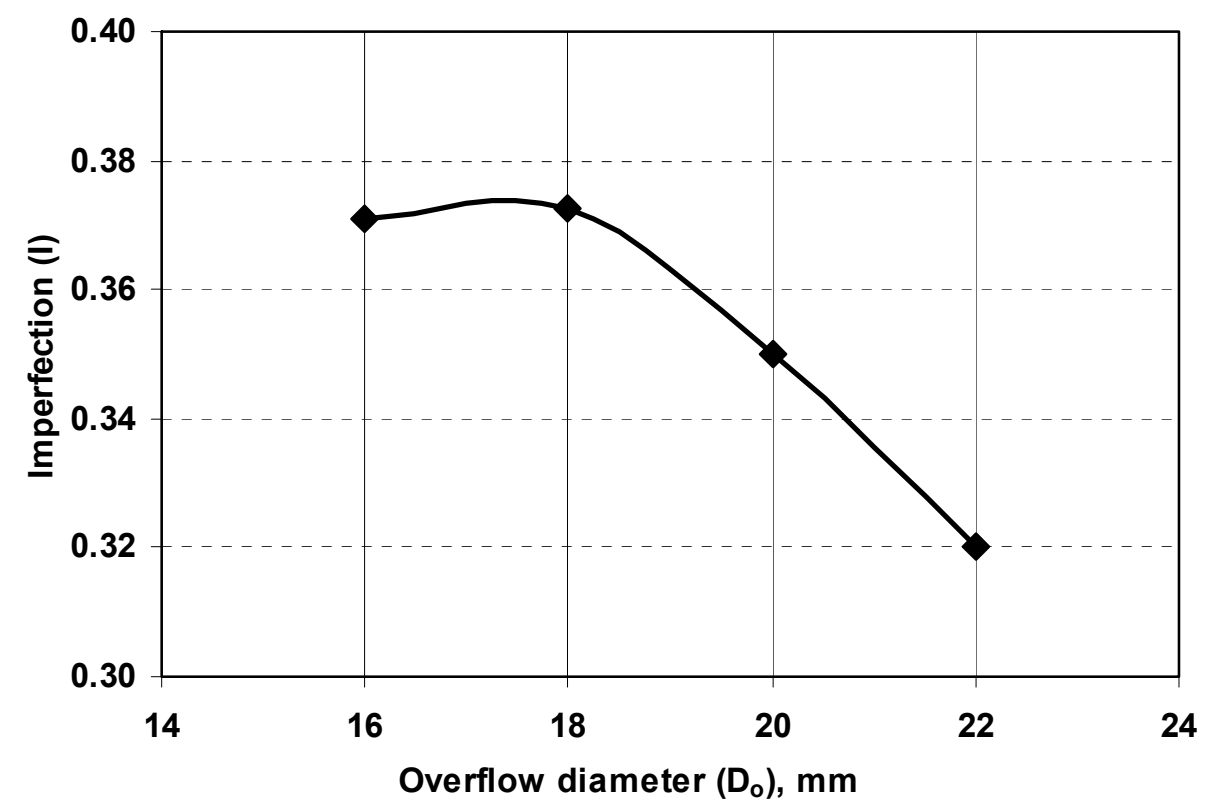

Fig. 7.62: Effect of overflow diameter on the imperfection.

\subsubsection{Effect of Overflow Diameter on the Solid Recovery and Solid Content.}

Figure 7.63 shows that the solid recovery in the underflow decreases gradually by increasing the overflow diameter. By increasing the overflow diameter, the volume split increase according to Eq. (7.5). This causes less suspension discharge to the underflow, which dominates the run of the solid recovery curve. 


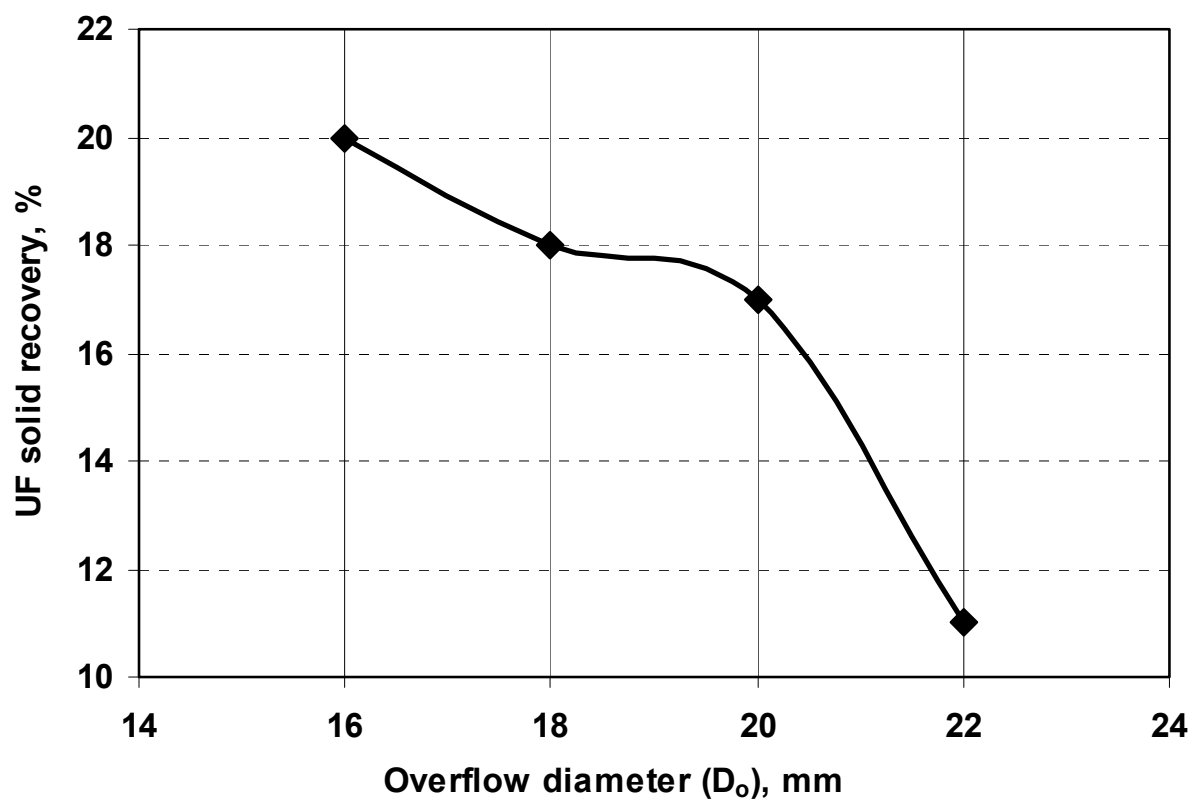

Fig. 7.63: Effect of the overflow diameter on the underflow solid recovery.

Fig. 7.64 shows the increase in the solid content by increasing the overflow diameter. As it was previously mentioned with increasing the overflow diameters, less suspension and accordingly less water will go through the underflow and thickened flow is expected. This results in an increase in the underflow solid content.

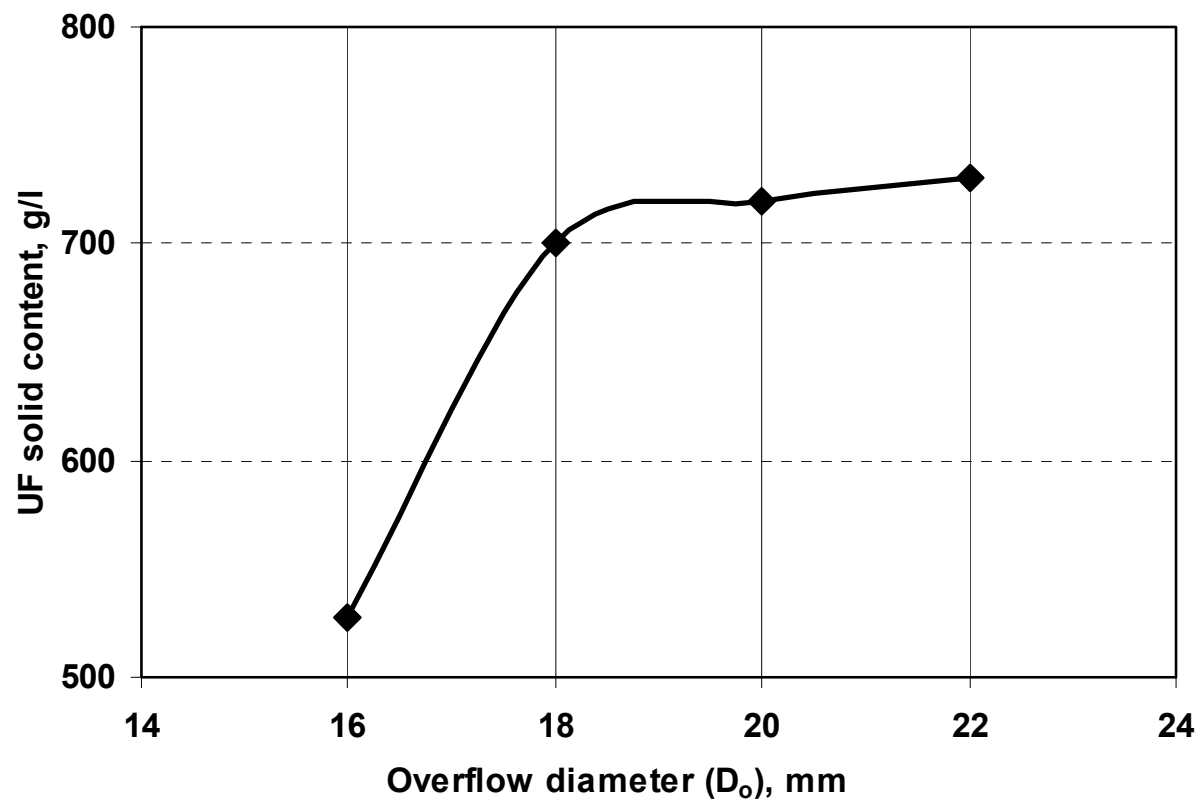

Fig. 7.64: Effect of overflow diameter on the underflow solid content.

\subsubsection{Effect of Feed Pressure (p)}

\subsubsection{Effect of Feed Pressure on the Separation Efficiency of the Fine Particles} $\left(\mathbf{T}_{0}, \mathbf{T}_{\min }\right)$ 
Figure 7.65 shows different separation curves obtained at different feed pressures. Figure 7.66 shows the effect of the feed pressure on the separation efficiency of the fine particles $\left(\mathrm{T}_{0}, \mathrm{~T}_{\min }\right)$.

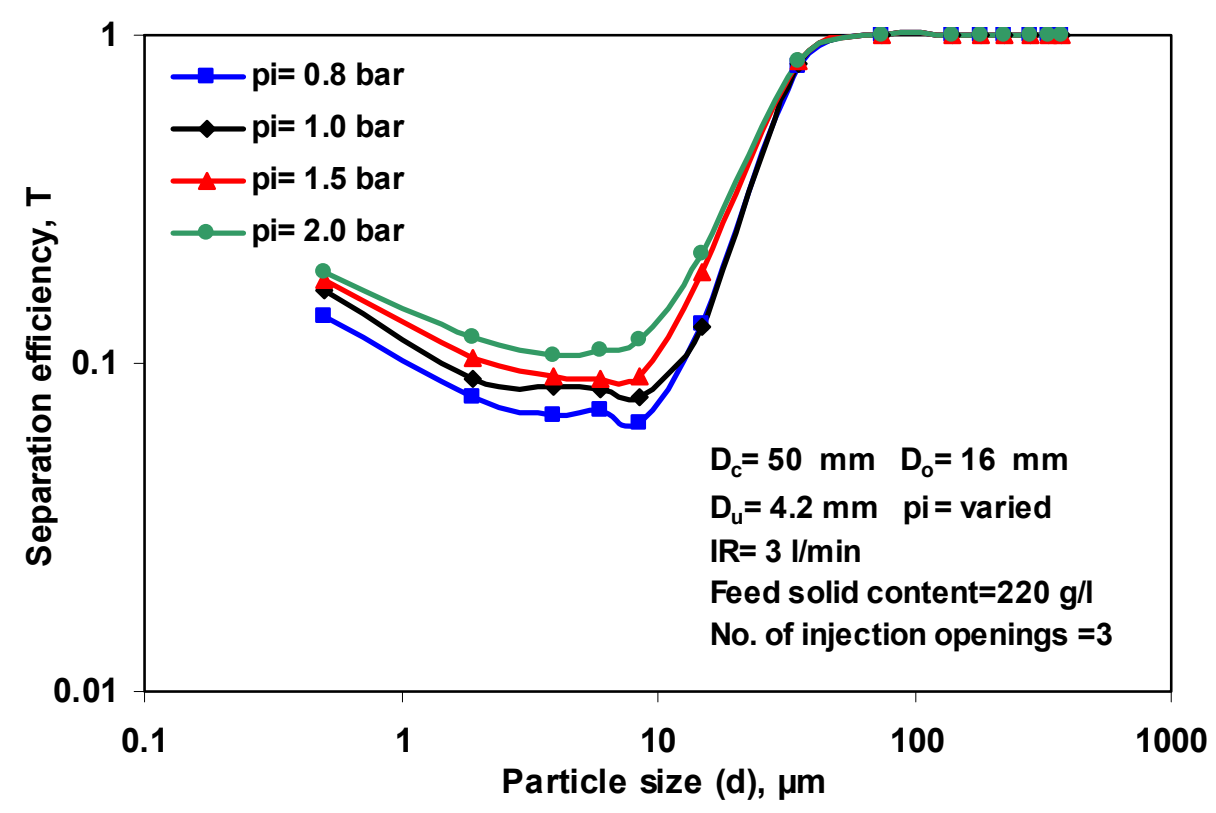

Fig. 7.65: Different separation curves at different feed pressures.

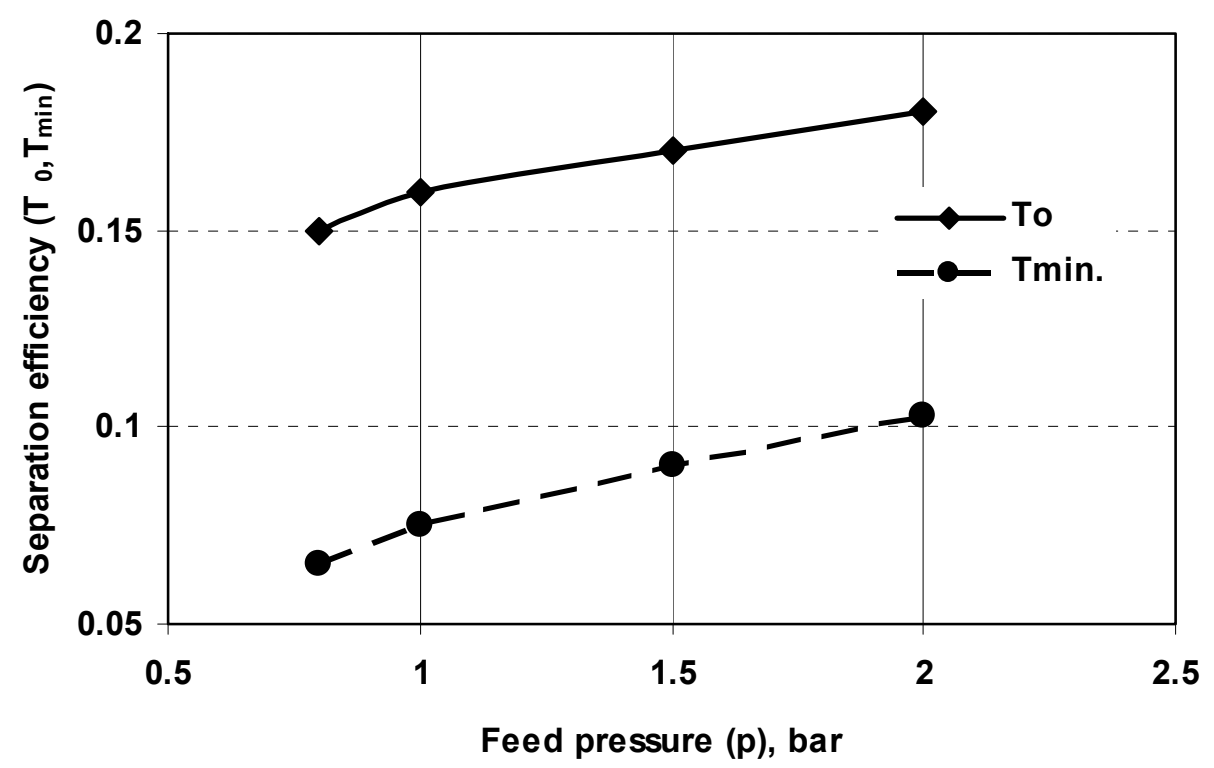

Fig. 7.66: Effect of the feed pressure on the separation efficiency of the fine particles $\left(\mathrm{T}_{0}, \mathrm{~T}_{\min }\right)$.

From Figure 7.65 and Fig. 7.66, it can be shown that the separation efficiency values increases slightly with increasing the feed pressure. This can be explained as follows; 
with increasing the feed pressure, the centrifugal force of the suspension will increase. This creates a high back pressure on the hydrocyclone wall from which the injection flow starts. The back pressure may reduce the injection velocity and then reduces the injection effect and the removal of the fines to the overflow. This results in an increase in the fines in the underflow at high feed pressure values. Therefore, a higher removal rate of fines is expected at low feed pressure. This result was also confirmed by Udaya et al. [89].

\subsubsection{Effect of Feed Pressure on the Cut size $\left(d_{50}\right)$ and Imperfection (I).}

Figure 7.67 and Fig 7.68 show the effect of the feed pressure on the cut size and the imperfection values respectively.

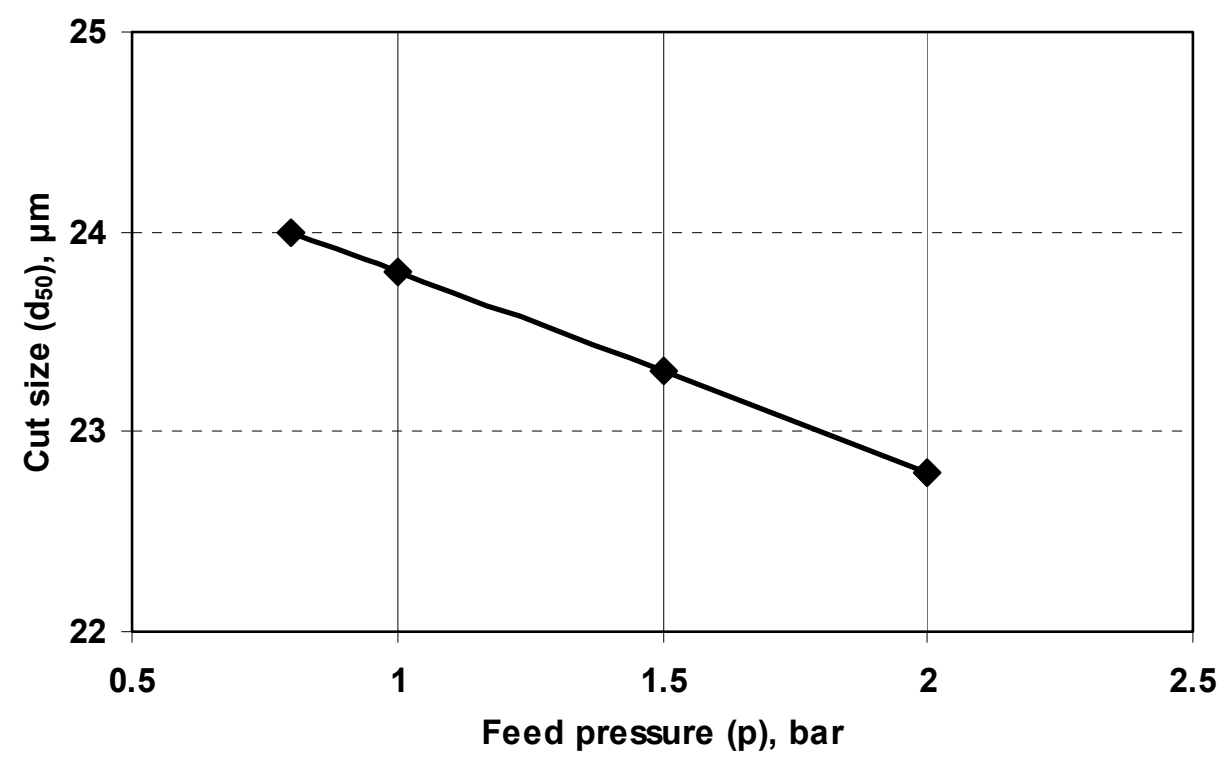

Fig. 7.67: Effect of the feed pressure on the cut size $\left(d_{50}\right)$.

Figure 7.67 shows that the cut size decreases slightly with increasing the feed pressure. This can be clearly explained in relation with Eq. (3.3) from which the feed pressure slightly influences the cut size $\left(\mathrm{d}_{50}\right)$ (exponent of - 0.25$)$.

As can be seen from Fig. 7.68 the imperfection value increases with increasing the feed pressure. This can be explained by Eq. (1.2) due to the decrease in the cut size $\left(\mathrm{d}_{50}\right)$. 


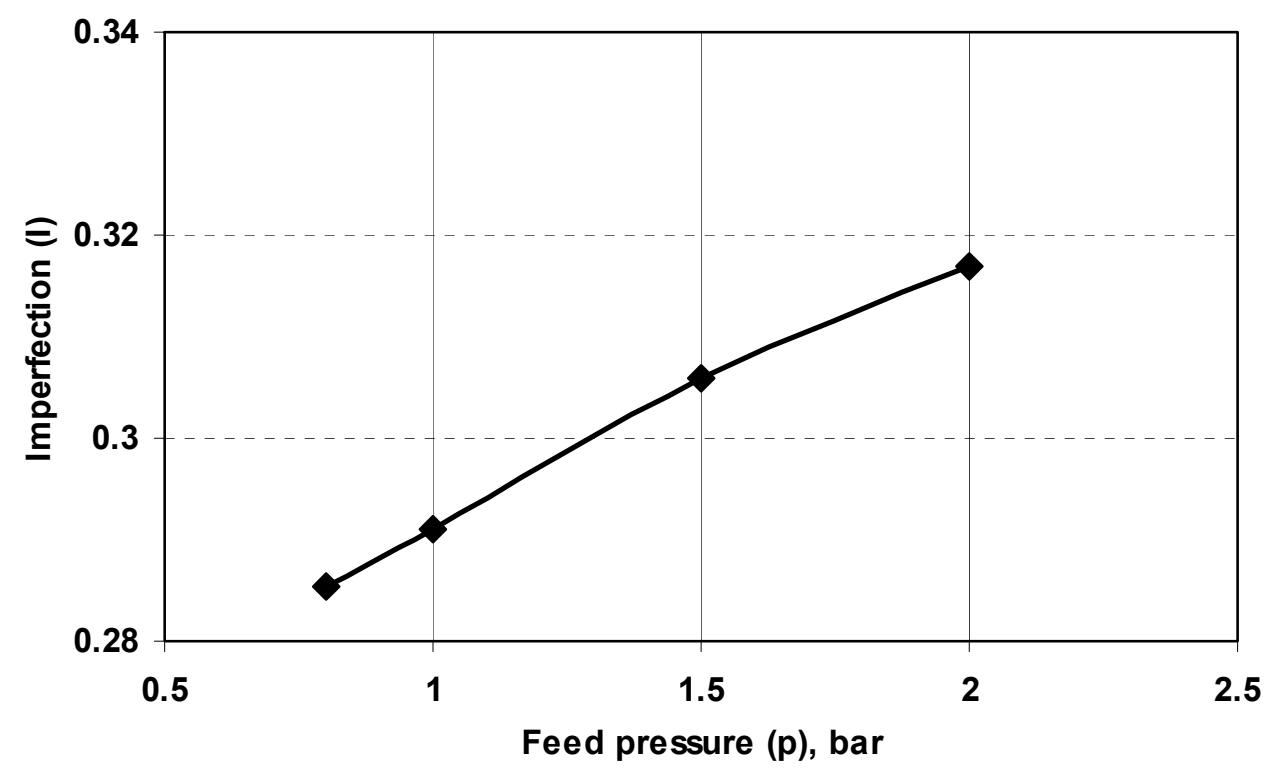

Fig. 7.68: Effect of feed pressure on the imperfection.

\subsubsection{Effect of Feed Pressure on the Solid Recovery and Solid Content}

Figure 7.69 shows the effect of the feed pressure on the underflow solid recovery.

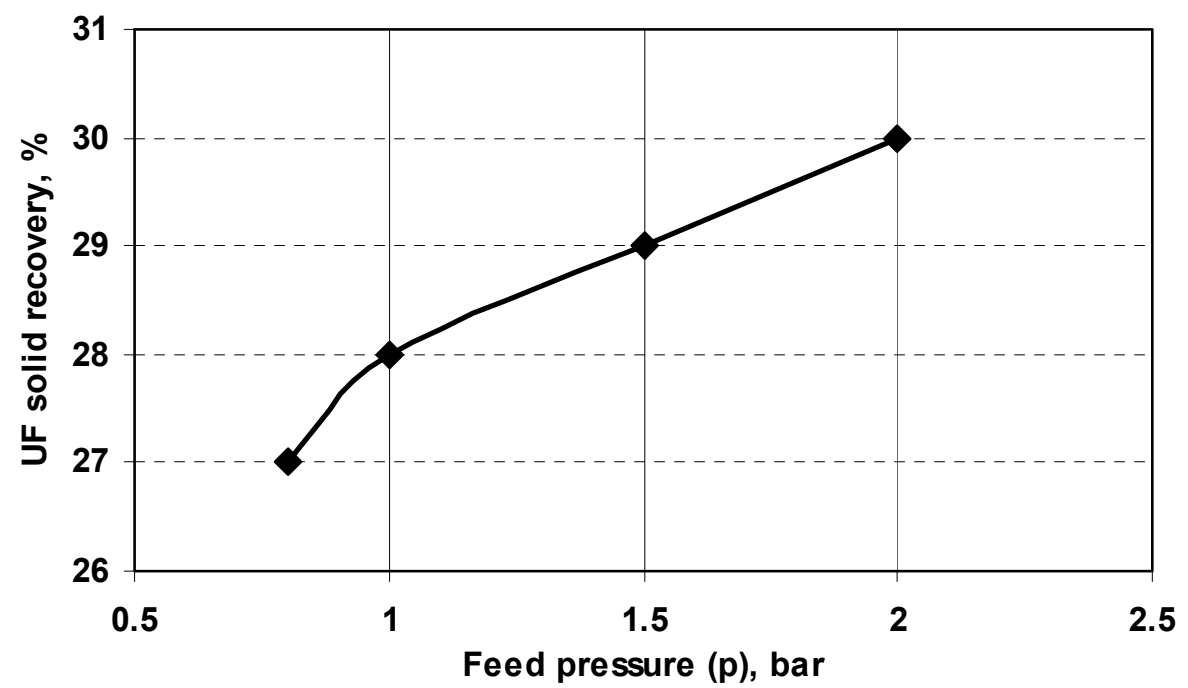

Fig. 7.69: Effect of feed pressure on the underflow solid recovery.

From Fig. 7.69 it can be indicated that the underflow solid recovery increases with increasing the feed pressure. This may be due to the increase in the feed throughput (capacity) which is directly proportional to the feed pressure as can be seen from Eq. (7.1). On the other hand, the solid recovery may increase due to the decrease in the 
cut size $\left(\mathrm{d}_{50}\right)$ as it was previously shown in Eq. (3.3) which means that more solids go to the underflow.

Figure 7.70 shows that the solid content increases sharply from $430 \mathrm{~g} / 1$ to $650 \mathrm{~g} / \mathrm{l}$ as the feed pressure increases from 0.8 to 1 bar. By further increase in the feed pressure up to 2 bar, the solid content is nearly constant. Due to the high pressure, the coarse particles will be dragged more to the wall of the cyclone under the high centrifugal force, and then downward to the underflow. In this case, the underflow discharge will consist mostly of the coarsest particles, and hence, a small percentage of the fines can be entrained. This leads to a thick underflow discharge. In this case, the apex reached its maximum discharge capacity so that the further increase in the feed pressure has no effect and a stable solid content is obtained.

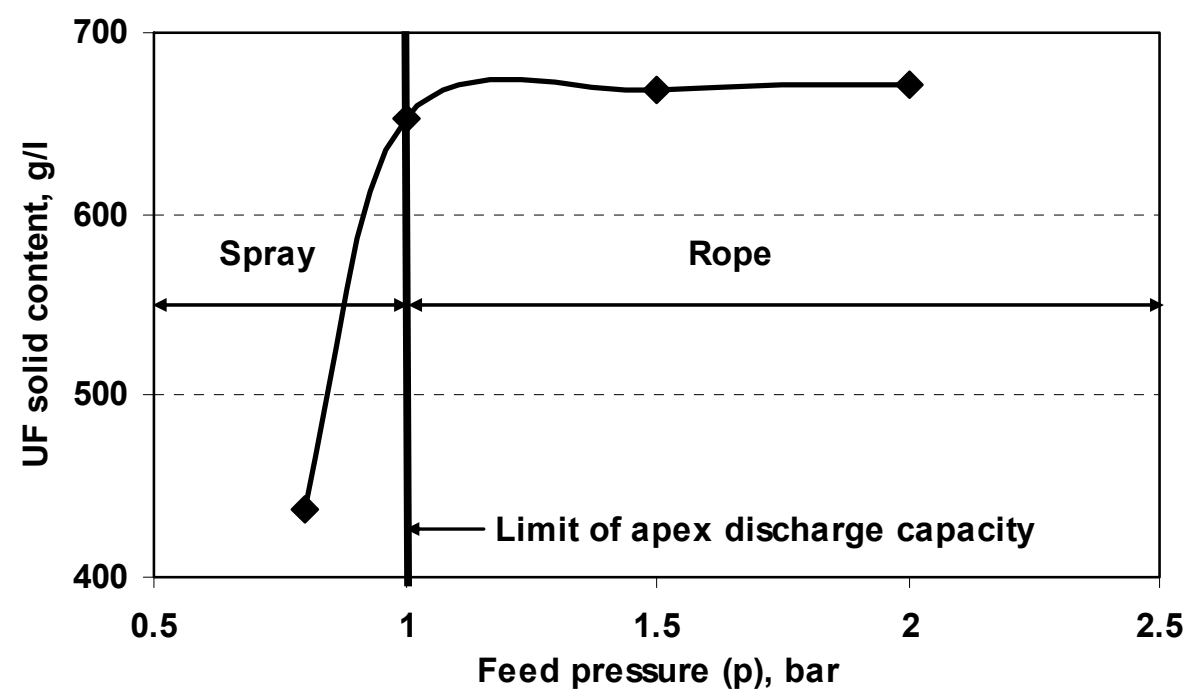

Fig. 7.70: Effect of feed pressure on the underflow solid content.

\subsubsection{Effect of Feed Solid Content}

\subsubsection{Effect of Feed Solid Content on the Separation Efficiency of the Fine} Particles

$\left(\mathrm{T}_{\mathbf{0}}, \mathrm{T}_{\min }\right)$.

Figure 7.71 shows different separation curves obtained at different feed solid contents. 


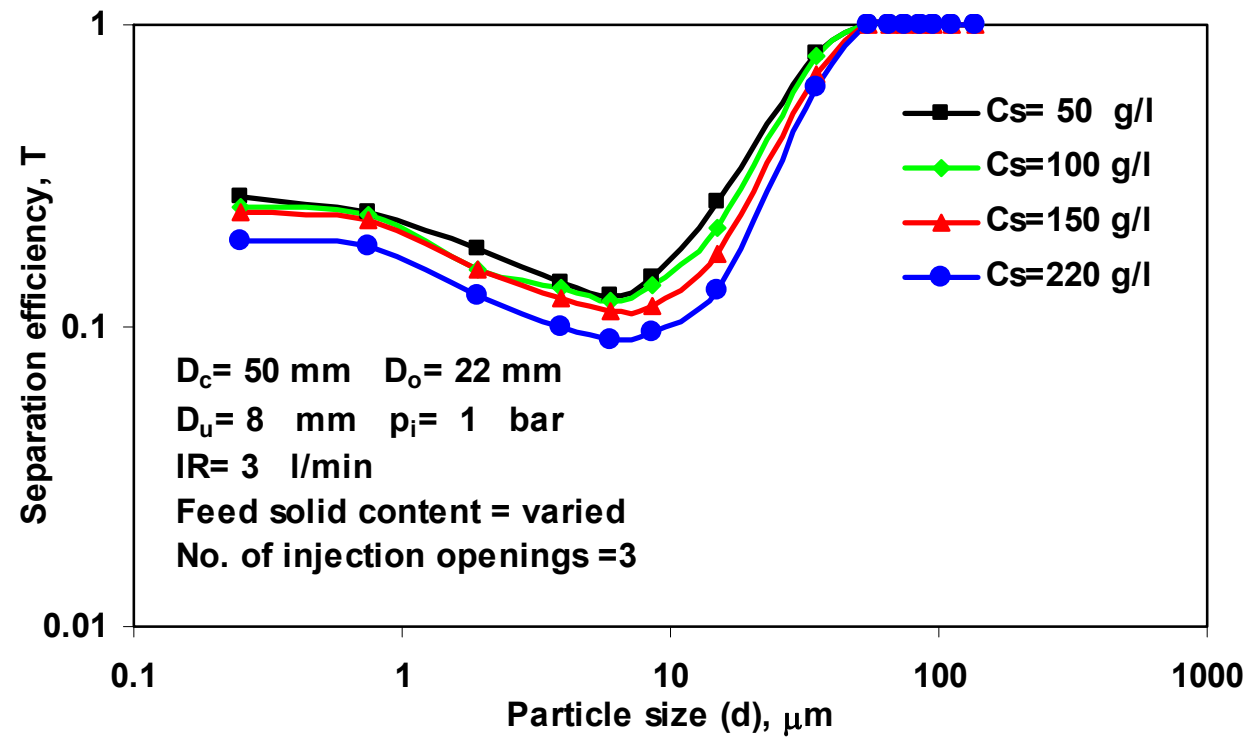

Fig. 7.71: Different separation curves at different feed solid contents.

Figure 7.72 shows the effect of the feed solid content on the separation efficiency values of the fine particles.

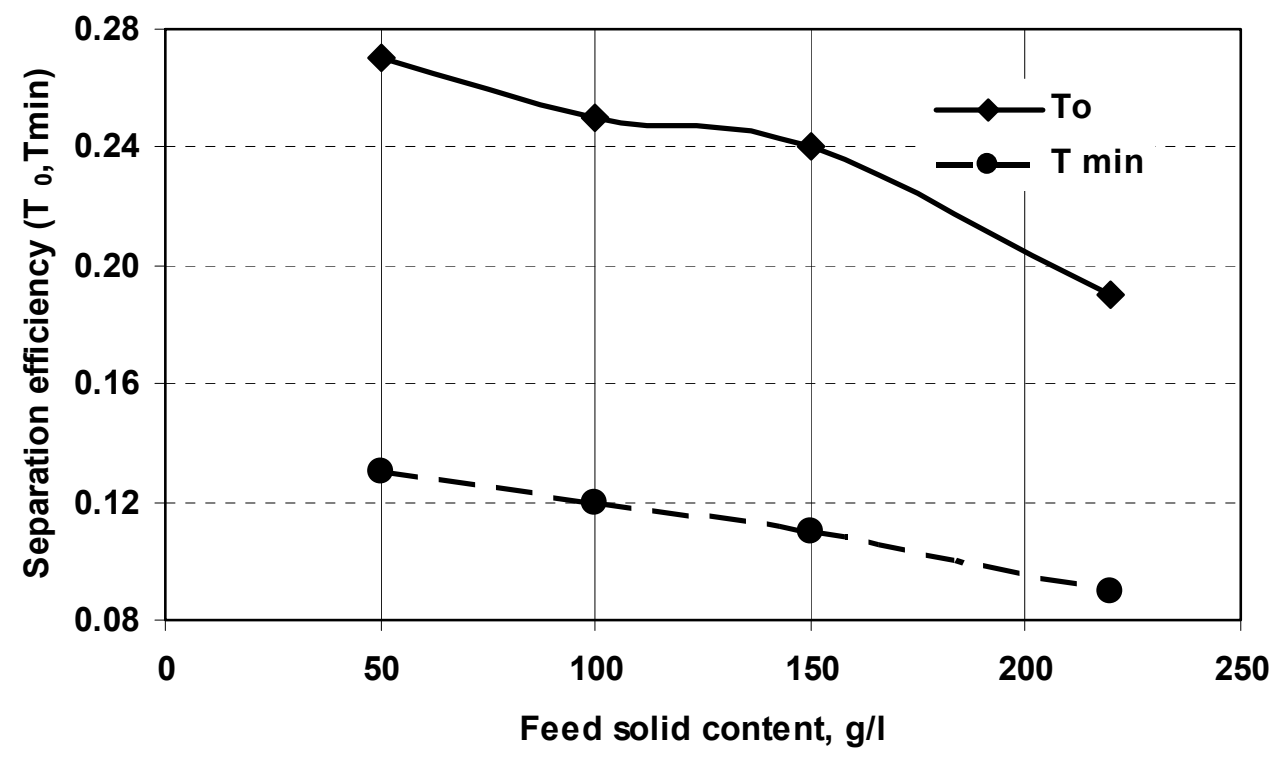

Fig. 7.72: Effect of the feed solid content on the separation efficiency of the fine particles $\left(\mathrm{T}_{0}, \mathrm{~T}_{\min }\right)$.

From Fig. 7.71 and Fig 7.72, it can be shown that the separation values decrease with increasing the feed solid content $(\mathrm{g} / \mathrm{l})$ which means that the higher the feed solid content, the more effective is the injection effect, and the lower is the percentage fines in the underflow. This can be explained as follows: 
At low feed solid content, the feed becomes more diluted and more feed water goes to the underflow. This will result in more fines to go to the underflow and then the washing effect of the injection flow will be decreased. At high feed solid content, the feed water in the underflow which must be displaced with the injected water will be less, and then the injection effect will be more effective as can be seen from Eq. (4.18). Further, the solids will be concentrated in the cone part. The concentrated particles will have higher chance to be subjected to the injection flow compared with their chance in the case of the diluted feed where the particles discharged rapidly to the underflow due to the dilution.

\subsubsection{Effect of Feed Solid Content on the Cut Size $\left(d_{50}\right)$ and Imperfection (I).}

Figure 7.73 shows that the cut size $\left(\mathrm{d}_{50}\right)$ increases gradually by increasing the feed solid content.

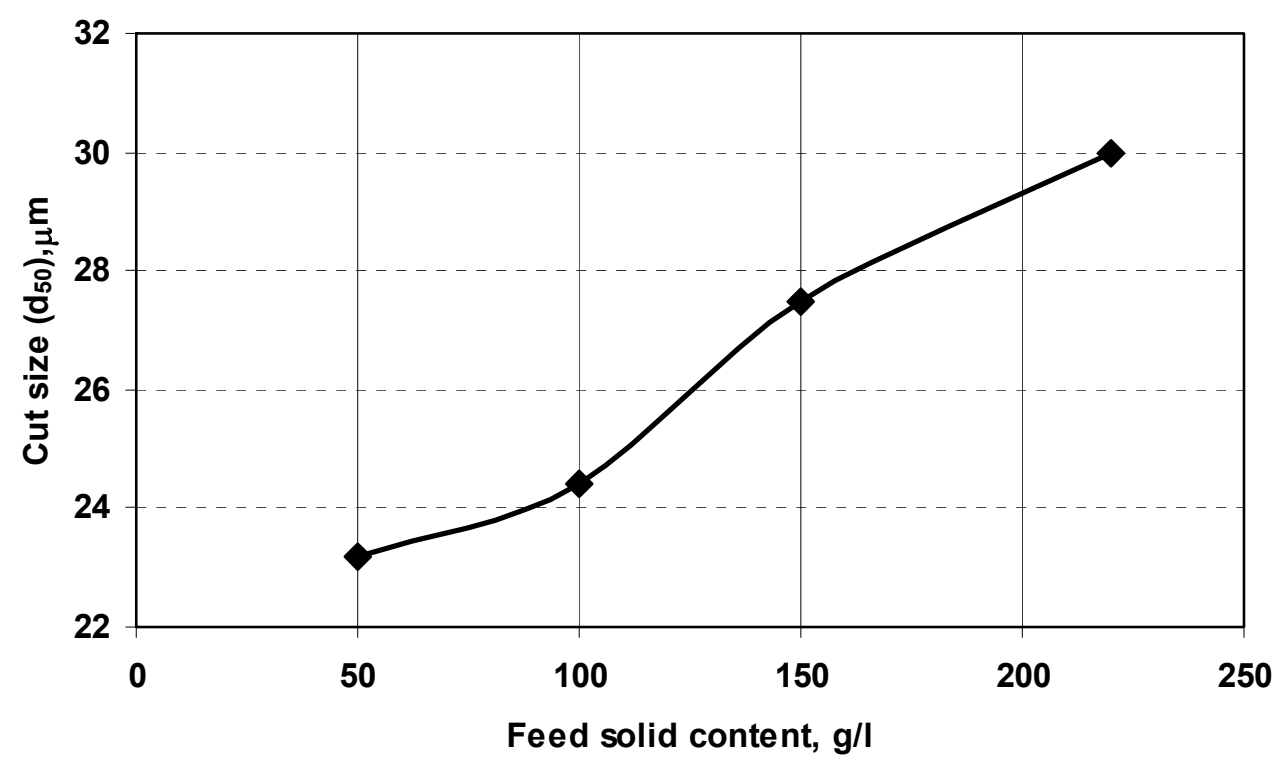

Fig. 7.73: Effect of feed solid content on the cut size $\left(\mathrm{d}_{50}\right)$.

This can be explained according to Eq. (3.3) where the cut size $\left(\mathrm{d}_{50}\right)$ is directly proportional to the feed solid content $\left(\varphi_{p}\right)$. So, the higher the feed solid content; the higher is the cut size $\left(\mathrm{d}_{50}\right)$ values.

Figure 7.74 shows that the imperfection decreases with increasing the feed solid content. Without injection, the imperfection normally tends to be increased. This 
decrease can be understood according to the gradual increase in the cut size $\left(d_{50}\right)$ where the imperfection is inversely proportional to the cut size $\left(\mathrm{d}_{50}\right)$ according to Eq. (1.2).

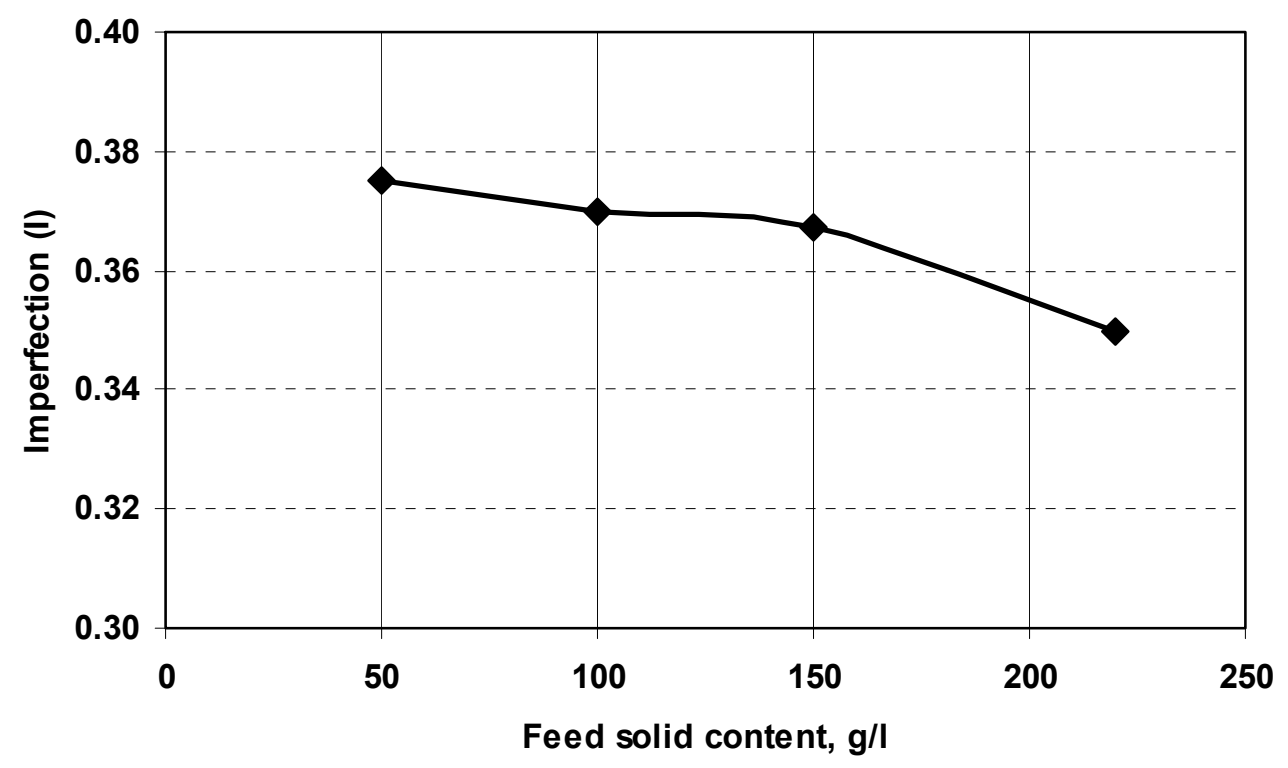

Fig. 7.74: Effect of feed solid content on the imperfection.

\subsubsection{Effect of Feed Solid Content on the Solid Recovery and Solid Content}

Figure 7.75 and Fig. 7.76 show the effect of changing the feed solid content on the underflow solid recovery and the solid content, respectively.

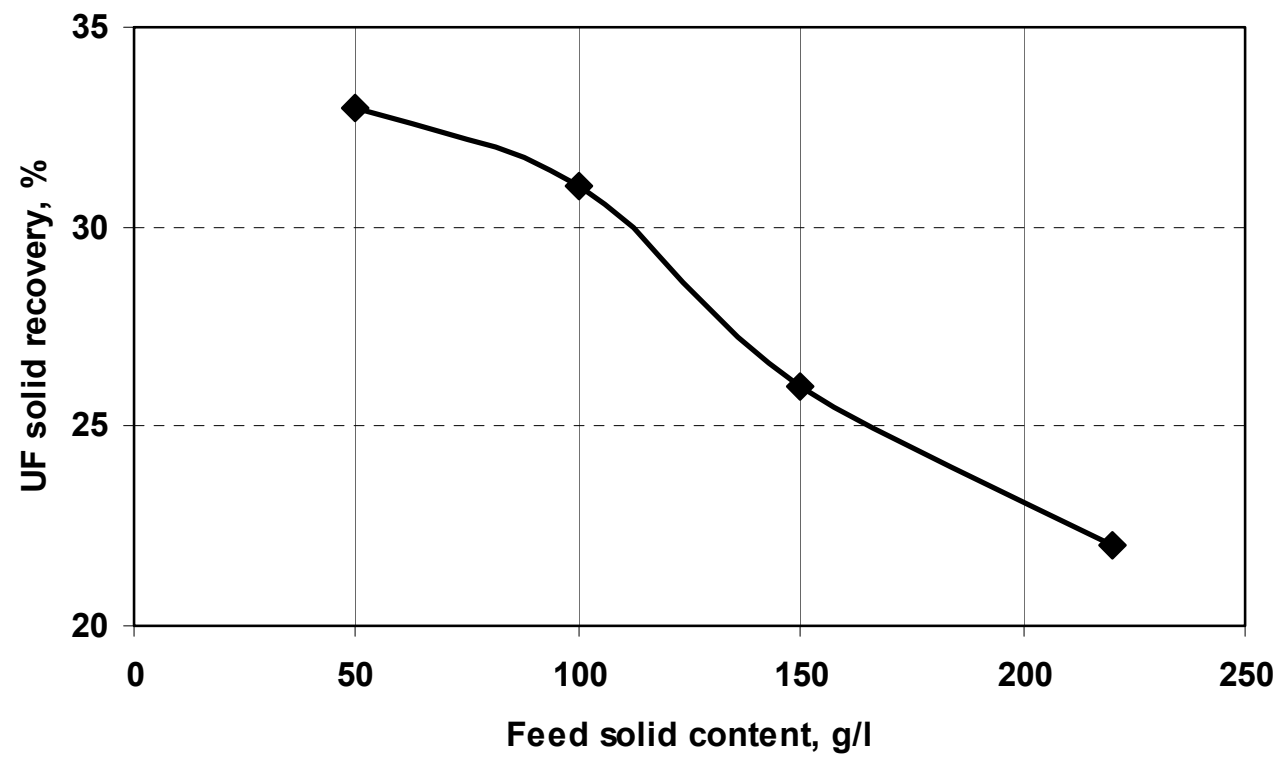

Fig. 7.75: Effect of the feed solid content on the underflow solid recovery. 


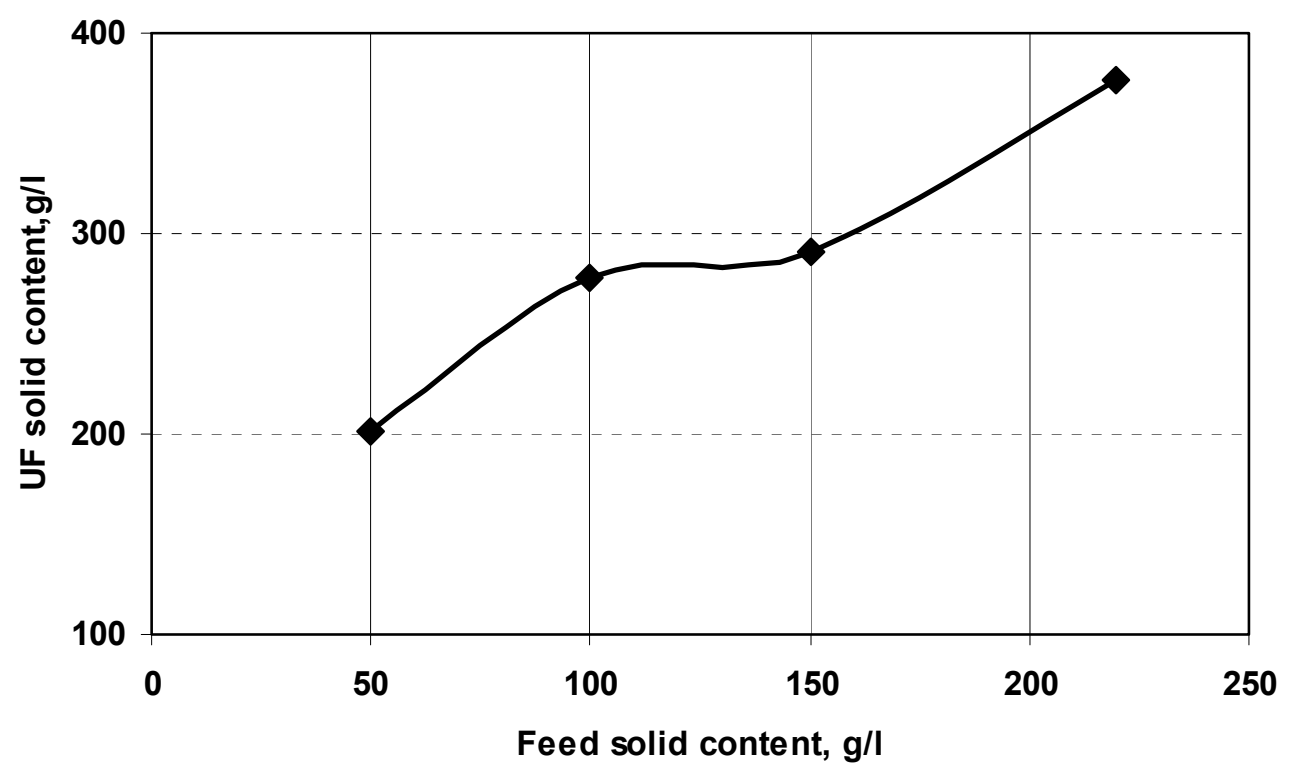

Fig. 7.76: Effect of feed solid content on the underflow solid content.

Figure 7.75 shows that the solid recovery in the underflow decreases with increasing the feed solid content. This may be due to the low discharge capacity of the apex at high feed solid content which will reduce the solids flow to the underflow. This can be also explained with the shape of the underflow discharge, which was previously shown in Fig. 3.4. The underflow discharge state can be summarised as follows: In dilute flow separation (low solid content), the underflow discharge takes place in spray form and a continuous air core can be observed which extends to the underflow. This separation presents high solid recovery as an advantage but at low solid content and high fine particles in the discharge which is a disadvantage as can be seen from Fig. 7.76. In dense flow separation (high solid content), more solids are stored in the conical part of the hydrocyclone and are partially forced to the overflow, consequently reducing the solids recovery to the underflow [5]. In this case, the air core is not going through the underflow apex due to the stored solids in the conical part above the apex and it oscillates intensively. Then the underflow starts changing from spray discharge to rope discharge which is characterized by high solid content [5]. The effect of rheology, which should also be taken into consideration, plays a role in these cases. 


\subsection{Controlled Water Injection}

From the forgoing discussed results concerning the investigated parameters, it can be seen that the water injection at the apex is sensitive to variations in the feed. Higher feed solid contents and/or coarser feeds will change the underflow solid content as well as the wash effect. Therefore, the injection must be controlled to stabilize the optimal injection rate. This control is based on the measurement of the discharge shape of the underflow which is dependent on the feed properties and the injection rate. Figure 7.77 depicts the hydrocyclone control principle of the water injection.

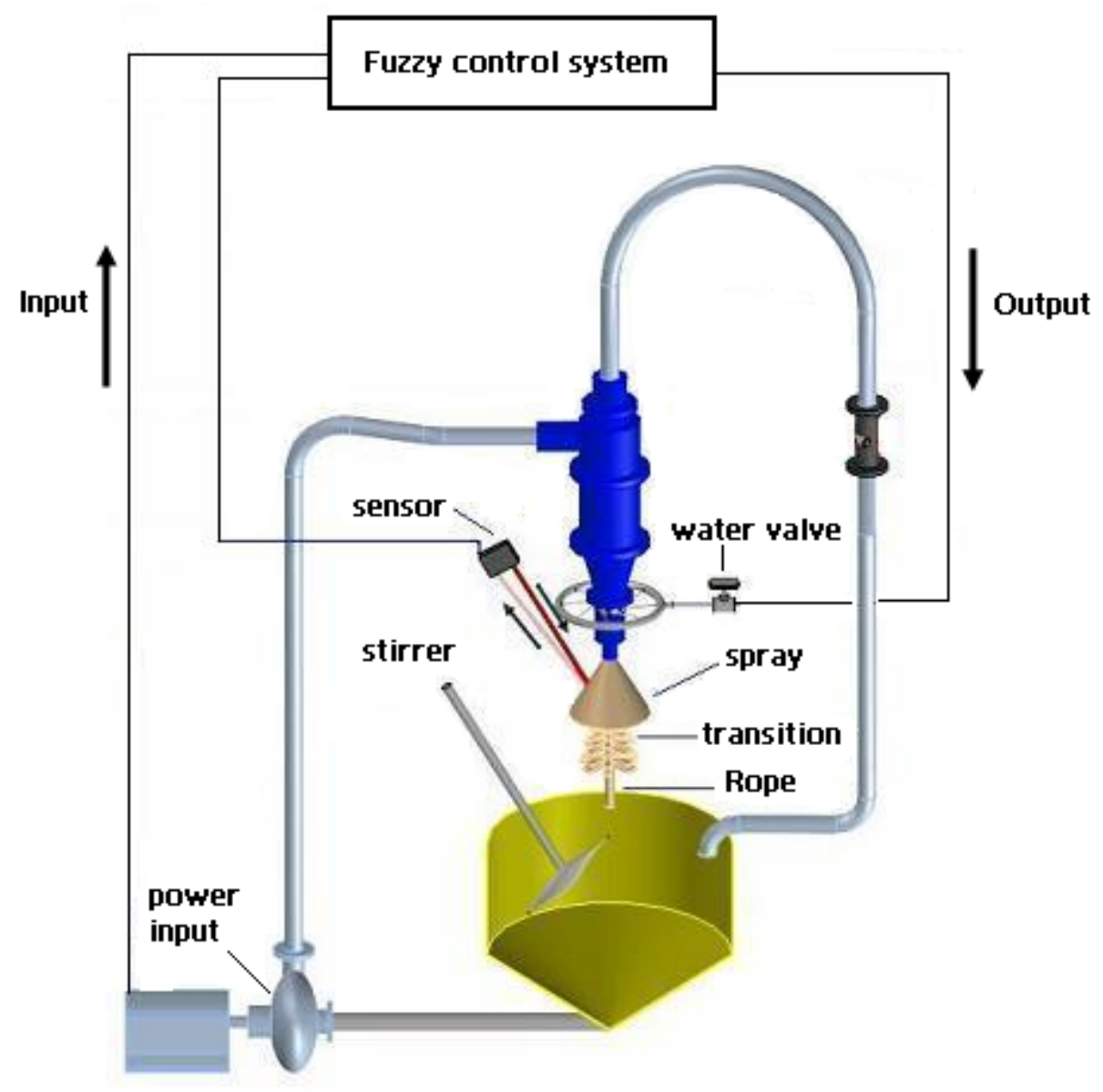

Fig. 7.77: Schematic of the controlled wash water injection to the hydrocyclone

The sensor, which monitors the underflow stream, is connected to the control database. Signals from the optical sensor, which indicate the shape and time behavior of the underflow, are transmitted to the interface together with the pump power input and the feed pressure. A valve in the water injection pipe performs the control action. 
As the water injection increases, the spray angle of the underflow gains higher values. To operate with high solids content in the underflow, the cyclone must be stabilized at a small spray angle not far from the transition point spray/rope. the control interval, signal and response, is $0.3 \mathrm{sec}$.

Finally, it can be concluded that this work provides fundamental data base and establishes reasonable preconditions for the application of controlled injection in the hydrocyclone apex. The controlled water injection hydrocyclone is now in a situation that makes it possible to estimate the optimum spray angle of the underflow discharge at which an optimum washing conditions is achieved. 


\section{Summary and Conclusions}

In hydrocyclones, the classification efficiency is limited by the fines, which are discharged together with the water in the underflow. Water injection in the conical part of the cyclone was tried by many researchers to reduce the fines in the underflow and increase the sharpness of the separation. Up to now, the application of water injection in cyclones has been restricted to special cases and separations in the coarse range. Most of the trials succeeded in reducing the fines in the underflow but with an increase in the cut size $\left(\mathrm{d}_{50}\right)$. Furthermore, the water injection was not tested for the small cyclone scale of $50 \mathrm{~mm}$. A further disadvantage is its sensitivity to changing feed conditions.

Therefore, the present work succeeded in developing a new method of water injection to hydrocyclones which is characterized by injection in the lowest possible point at the end of the apex nozzle where the sediment is stored just before discharging to the underflow. For fine particle displacement to the inner vortex of overflow, it is necessary to operate with an injection velocity $\left(V_{i n}\right)$ that overcome the tangential velocity $\left(V_{\text {tang }}\right)$ of the feed flow. The injection velocity is determined by the water injection rate $I R$, the diameter $d_{i n}$ of the injection channel, and the number of injection openings $n$ spaced equally around the circumference according to the following formula:

$V_{i n}=4 I R / n \pi d_{i n}^{2}$

To determine empirically the feed tangential velocity at different inlet pressures, the $50 \mathrm{~mm}$ cyclone was operated with only water as feed without water injection. Empirical equations which describe the effect of the feed pressure (bar) on the water flow rate $(1 / \mathrm{min})$ through the feed, overflow, and underflow were obtained. Using these equations, the feed tangential velocity can be calculated for every feed pressure. Then, the water was injected to study the effect of the injection rate on the water distribution through the overflow and underflow openings. It was found that a certain percentage of the injected water goes to the overflow stream. This portion of water increases with increasing the injection rate which is the desired effect to carry the 
entrapped fines to the overflow. The experiments showed also that at low injection rates, the underflow rate increases while at higher injection rates it decreases. The effect of the injection rate on the volume split parameter was investigated at low and high injection velocities by using five and three injection openings, respectively. It was found that at high injection velocity (up to a certain limit) the volume split parameter increases gradually with increasing the injection rate which improves the injection effect. At low injection velocity, the volume split parameter decreases at the beginning of the injection and then increases with further increase in the injection rate.

After that, the classification experiments with water injection were carried out using a kaolin/feldspar suspension with solid content about $220 \mathrm{~g} / \mathrm{l}$. The dependency of the fine percent in the underflow product on the different design and operating parameters of the $50 \mathrm{~mm}$ water injection hydrocyclone were investigated. These parameters were: injection geometry, injection rate, hydrocyclone geometry, hydrocyclone operation, and feed conditions. The effects of these parameters on the characteristics of the separation process such as the cut size $d_{50}$, the separation sharpness, the underflow solid recovery, and the underflow solid content were evaluated.

The results showed that the tangential injection in the direction of the main flow inside the hydrocyclone at the lowest possible point above the hydrocyclone apex results in a greater washing effect without changing the overflow quality. Moreover, the injection may lead even to lower cut sizes due to the dilution, which diminishes particle hindrance during the settling of the coarse particles. This result is in contradiction with former investigations. The most important implication from the results is the existence of an optimum injection velocity of about $9.5 \mathrm{~m} / \mathrm{sec}$ at which the maximum washing effect is achieved. The optimum injection velocity $(\mathrm{m} / \mathrm{sec})$ can be obtained by varying the injection rate, the number of injection openings, or the injection opening diameter, respectively. Therefore, a compromise between the injection rate and a high number of injection points leads to better ax-symmetric flow conditions and better washing effect.

Both the systematic and the statistical errors were estimated. The statistical analysis showed that the experimental results are reproducible with a maximum relative error 
of 7 percent at 95 percent confidence limits. The level of the systematic error is reduced using experimental corrections.

The experimental data were simulated based on the separation model of Schubert and Neesse. The simulation results showed a reasonable agreement with the experimental ones especially in the range of low injection velocities up to $5 \mathrm{~m} / \mathrm{sec}$, while at high injection velocity $(9.5 \mathrm{~m} / \mathrm{sec})$ more turbulence is produced which is not considered by the model.

The simulation results indicated that the effect of the injection on the fine particle separation in the underflow can be summarized as a combination of two phenomena. First at low injection rates, the counter current effect caused by the injection flow dominates until an optimum injection rate is reached. Then, at higher injection rates, the effect of high turbulence diffusion becomes dominant, and disturbs the separation.

Analyzing the results obtained using the $50 \mathrm{~mm}$ hydrocyclone with water injection, showed that the injection at the apex is sensitive to variations in the feed characteristics. Higher feed solid contents and/or coarser feeds will change the underflow solid content as well as the wash effect. Therefore, the injection must be controlled to stabilize the optimal injection rate. This control is based on the measurement of the discharge shape (angle) of the underflow which is dependent on the feed properties and the injection rate.

Finally, it can be concluded that the results of the present work provide a fundamental data base and establishes reasonable preconditions for the application of controlled water injection in the hydrocyclone apex. The controlled water injection hydrocyclone is now set up and runs under stable operating conditions. This is the recommendation of the present work for the future and further work concerning the water injection into the hydrocyclone apex.

Future and further work should complement the present results and continue developing the water injection hydrocyclone and improve the controlling system to respond to regular and irregular changes. 


\section{Bibliography}

[1] Schubert, H. Neesse, Th. 1980: A Hydrocyclone Separation Model in Consideration of the Turbluent Multi-Phase Flow, Int. Conference on Hydrocyclone, Cambridge.

[2] Frachon, M. and Cilliers, J.J. 1999: A general model of hydrocyclone partition curves, Chemical Engineering Journal, Vol.73, 53-59.

[3] Lynch, A.G. 1977: Mineral crushing and grinding circuits, Elsevier Scientific, New York, 105-120.

[4] Terra, A. 1938: Essay d'un theorie de lavage, Rev. Ind. Miner., mem. 18.

[5] Eder, Th. 1961: Problem der Trennscharfe. Aufbereit. - Tech., 2(3), 104-109; 2(4), 136-148; 2(8), 313-321; 2(12), 484-495.

[6] Neesse, Th., Golyk, V., Kaniut, P., Reinsch. 2004: Hydrocyclone control in grinding circuits, Minerals Engineering, Vol. 17, 1237- 1240.

[7] Bradley, D., 1956: United Kingdom Atomic Energy Report, AERE CE/M 177.

[8] Heiskanen, K. 1993: Particle Classification, Chapman \& Hall Press, London, $321 \mathrm{pp}$.

[9] Bradley, D. 1965: The Hydrocyclone, Pergamon Press, Oxford, 330 pp.

[10] Dueck, J., Pikushchak, E., Minkov, L., Farghaly, M., Neesse, Th. 2009: Simulation of Water Injection in Hydrocyclones, Physical Separation 09 Conference, Falmouth, United Kingdom.

[11] Lin, I.J. 1987: Hydrocycloning thickening: dewatering and densification of fine particulates, Separat. Sci. Technol. 22, 1327-1347.

[12] Klima, M. S., Kim, B. H. 1998: Dense-medium separation of heavy-metal particles from soil using a wide angle hydrocyclone, J. Envirson. Sci. Health Part A 33, 1325-1340.

[13] Anonymous 1997: Hydrocyclomne separation in the field of environmental technology, Chem. Biochem. Eng. Q. 11, 157. 
[14] Klima, M.S. and Kim, B. H. 1997: Multi-stage wide-angle hydrocyclone circuits for removing fine, high density particles from a low density soil matrix, J. Envirson. Sci. Health Part A 32, 715-733.

[15] Mainza, A., Powell, M.S., Knopjes, B. 2004: Differential classification of dense material in a three-product cyclone, Minerals Engineering, Vol. 17, 573-579.

[16] Peachy, B.R., Solanki, S.C., Zahacy, T.A., Peirs, K. 1998: Downhole oil/water separation moves into high gear, J. Can. Petrol. Technol, 7, 34-41.

[17] Anonymous 1996: Hydrocyclones- cleaning oily water, Oil Gas-Eur. Mag. 3, 46.

[18] Tong, H., Yong, W. 1996: Development and field trial of a new deoiling Hydrocyclone, Oil Gas-Eur. Mag. 1, 29-31.

[19] Anonymous 1996: Hydrocyclones for oil from water separation, Filt. Separat. 33, 291-292.

[20] Young, G.A.B., Wakley, W.D., Taggart, D.L., Andrews, S.L, Worrell, J.R. 1994: Oil-water separation using hydrocyclones - an experimental search for optimum dimensions, J. Petrol. Sci. Eng. 11, 37-50.

[21] Adupeasah, S.P., Diosady, L.L., Rubin, L.J. 1993: A multistage hydrocyclone stirred -tank system for counter current extraction of canola oil, J. Am. Oil Chem. Soc. 70, 755-762.

[22] Dickey, L.C., Dallner, M.F., Radewonuk, E.R., Parris, N., Kurantz, M., Craig, J.C. 1997: Hydrocyclone separation of dry milled corn, Cereal Chem. 74, 676680 .

[23] Singh, N., Eckhoff, S.R. 1996: Hydrocyclone in the wet corn wet milling industry, Cereal. Food World, 41, 676-680.

[24] Trim, D.S., Marder, R.C.1995: Investigation of hydrocyclones for concentration of cassava milk, Starch- Starke, 47, 306-311.

[25] Singh, N., Eckhoff, S.R. 1995: Hydrocyclone procedure for starch- protein separation in laboratory wet- milling, Cereal Chem. 72, 344-348.

[26] Dhamo, N. 1996: Electrochemical oxidation of cyanide in the hydrocyclone cell, Waste Manage. 16, 257-261. 
[27] Dhamo, N. 1994: An electrochemical hydrocyclone cell for the treatment of dilute solution - approximate plug-flow model for electrodeposition, J. Appl. Elechtrochem., 24, 745-750.

[28] Cilliers, J.J, Harrison, S.T.L.: 1997: The application of many hydrocyclones in the concentration of yeast suspension, Chem. Eng. J., 65, 21-26.

[29] Yuan, H., Rickwood, D., Smyth, I.C., Thew, M.T. 1996: An investigation into the possible use of hydrocyclones for the removal of the yeast from beer, Biosepataion 6, 159-163.

[30] Bendixen, B., Rickwood, D. 1994: Effects of hydrocyclones on the integrity of animal and microbial cells, Bioseparation 4, 21-27.

[31] Li, M., Johnston, R., Xu, L., Filonenko, Y., Parker, I. 1999: Characterization of hydrocyclone-separated eucalypt fibre fractions, J. Pulp. Pap. Sci., 25, 299-304.

[32] Wood, J.R., Grondin, M., Karnis, A. 1991: Characterization of mechanical pulp fines with a small hydrocyclone; 1 . The principle and nature of the separation, J. Pulp. Pap. Sci., J1-J5.

[33] Driessen, M.G. 1951: Theory of flow in a cyclone, Rev. L'Industrie Min. Spl., $449-461$.

[34] Obeng, D.B., Morrell, S. 2003: The JK three-product cyclone- performance and potential applications, International journal of mineral processing 69, 129-142.

[35] Wills, B.A. 1992: Mineral Processing Technology, $5^{\text {th }}$ ed., Pergamon, Oxford.

[36] Dell Villar, R. and Finch, J.A. 1992: Modelling the cyclone performance with a size dependant entrainment factor, Miner. Eng. 5, 661-669.

[37] Bendraski, S. 1948b: Graphical and analytical interpretation of investigation results of the flow rate in hydrocyclones with variable process and design parameters. Proc. $2^{\text {nd }}$ Int. Conf.

[38] Lynch, A.J., Rao, T.C., Bailey, C.W. 1975: The influence of design and operating variables on the capacities of hydrocyclone classifier. International Journal of Mineral Processing 2, 29-37.

[39] Restarik, C.J., Krinic, Z. 1991: The effect of overflow/underflow ratio on dense medium cyclone operation. Minerals Engineering 4(314), 263-270. 
[40] Flintof, B.C., Plitt, L.R., Yurak, A.A. 1987: Cyclone modelling: a review of present technology. CIM Bulletin 80(905), 39-50.

[41] Monredon, T.C., Hsieh, K.T., Rajamani, R.K. 1992: Fluid flow model of the hydrocyclone: an investigation of device dimensions. International Journal of Mineral Processing 35, 65-83.

[42] Braun, T., Bohnet, M. 1990: Influence of feed solid concentration on the performance of hydrocyclones, Chem. Eng. Technol. 13, 15-20.

[43] Kelsall, D.F. 1952: A study of the motion of solid particles in a hydraulic cyclone. Proc. $1^{\text {st }}$ Int. Min. Proc. Congr., Recent development in Mineral Dressing, Institution of Mining and Metallurgy, London, 209-228.

[44] Rietema, K. 1961a: Performance and Design of Hydrocyclones, Parts 1-4, Chem. Eng. Sci., 15, 298-325.

[45] Fahlstrom, P.H. 1963: Studies of the hydrocyclone as a classifier. In Mineral Processing, Proc. $6^{\text {th }}$ Int. Min. Proc. Congr., Cannes (ed. A. Roberts), Pergamon Press, Oxford, 87-114.

[46] Dreissen, H.H. and Absil, J. 1981: Theory, practice and developments of the DSM heavy-medium cyclone process for Minerals. Trans. AIME, 270, 18641878.

[47] Rietema, K. 1962: Liquid- solids separation in a cyclone. The effect of turbulence in separation. Proc. Symp. On Interaction between Fluids and Particles, Inst. Chem. Engeers, London, 275-298.

[48] Neesse, Th., Schubert, H. 1975: Modellierung und verfahrenstechnische Dimensionierung der turbulenten Querstromhydroklassierung, Teil I, Chemische Technik, 27 (9), 529-533.

[49] Neesse, Th., Schubert, H. 1976: Modellierung und verfahrenstechnische Dimensionierung der turbulenten Querstromhydroklassierung, Teil II, Chemische Technik, 28 (2), 80-83.

[50] Neesse, Th., Schubert, H. 1976: Modellierung und verfahrenstechnische Dimensionierung der turbulenten Querstromhydroklassierung, Teil III, Chemische Technik, 28 (5), 273-278. 
[51] Neesse, Th., Schubert, H. 1976: Modellierung und verfahrenstechnische Dimensionierung der turbulenten Querstromhydroklassierung, Teil IV, Chemische Technik, 29 (1), 14-18.

[52] Neesse, Th., Espig, D., Schubert, H. 1976: Particle transfer from a turbulent gas steam to a sediment layer at the wall- a microprocess of cyclone separation, Preprints $1^{\text {st }}$ World Congress on Particle Technology, Nuremberg, Part IV, 2538.

[53] Neesse, Th., Espig, D., Schubert, H. 1984: Die Trennkorngroße des Hydrozyklon bei Dünnstrom und Dichtstromtrennungen. Preprints $1^{\text {st }}$ Europ. Sympos. Partikelklassierung in Gasen und Flussigkeiten, Nuremberg, H3-H18.

[54] Schubert, H., Bohme, St., Neesse, Th., Espig, D. 1986: Classification in turbulent two-phase flows. Preprints $1^{\text {st }}$ World Congress on Particle Technology, Nuremberg, Part IV, 419-442.

[55] Kerkhoff, T., Neesse, T. 1995: Rationelle Methodik für Hydrozyklonversuche mit kontaminiertem Material. Aufbereitungstechnik, Vol. 36 (4), 506-514.

[56] Neesse, T., Dueck, J., Kerkhoff, T. 1996: Feinstkornabscheidung im Hydrozyklon. Aufbereitungstechnik, Vol. 37 (9), 413-421.

[57] Kerkhoff, T., 1996: Feinkornklassierung in einem luftkernlosen Hydrozyklon. PhD Thesis. TF FAU, Erlangen.

[58] Neesse, Th., Gerhart, Ch., Bickert, G. 1997: Separation curves for the hydrocyclone with fishhook, Proceedings of the XX. Intern. Mineral Processing Congress, Aachen, GMDB, Clausthal-Zellerfeld, Vol. 2, 457-465.

[59] Neesse, Th., Schubert, H., Graichen, K. 1991: Practische und theoretische Aspekts der Dichstromklassierung. Aufbereit.- Tech., 32 (9), 459-472; preprints XVII IMPC, Dresden, Part 1, 23-42.

[60] Svarovsky, L. 1980: Critical Evaluation of the simple ways of determining the cut size. Proc. $1^{\text {st }}$ Int. Conf. Hydrocyclones, Cambridge, British Hydromechanics Research Association, Cranfield, Paper 4, 37-48.

[61] Plitt, L.R., Flintoff, B.C. and Stuffco, T.J. 1987: Roping in hydrocyclones. Proc. $3^{\text {rd }}$ Int. Conf. Hydrocyclones, Oxford, BHRA, (ED. P. Wood), Elsevier, Amsterdam, 21-34. 
[62] Concha, F., Barrientos, A., Montero, J., Sampio, R. 1994: Air core and roping in hydrocyclones. In: Proceedings of the $8^{\text {th }}$ European Symposium on Comminution, Stockholm, Sweden, 814-823.

[63] Dyakowski, T., Williams, R.A. 1995: Prediction of the air core diameter within a hydrocyclone. Int. J. Miner. Process. 40, 1-11.

[64] Barrientos, A., Sampaio, R., Concha, F. 1993: Effects of the air core on the performance of hydrocyclone. In. Proceedings of the XVIII International Mineral Processing Congress, Sidney, Australia, 267-270.

[65] Yopes, S.W., Spottiswood, D.J., Bull, W.R. and Pillai, K.J. 1987: Astudy of the effect of slurry rheology on hydrocyclone performance. Proc. $3^{\text {rd }}$ Int. Conf. Hydrocyclones, Oxford, BHRA (ed. P. Wood), Elsevier, Amsterdam, 221-234.

[66] Dyakowski, T., Hornung, G., Williams, R.A. 1994: Simulation of nonNewtonian flow in hydrocyclone. Trans. J. Chem. E., Part A, 513-520.

[67] Dueck, J., Matvienko, O., Neesse, Th. 1999: Hydrodynamics and particle separation in the hydrocyclone. In: Calata, G.P., Di Marco, P., Shah, R.K. (Eds.), Two Phase Flow Modelling and Experimentation. Edition ETS, Psi, Italy.

[68] Dueck, J., Matvienko, O., Neesse, Th. 2000: Modelling of hydrodynamics and separation in a hydrocyclone. Theor. Found. Chem. Eng. 34, 428-438.

[69] Dueck, J., Matvienko, O., Neesse, Th. 2001: Numerical Modelling of hydrocyclone dynamics for process control. In: Shiaotung-Chiang, Lee, S.E., Advance in Filtration and Separation, for the $21^{\text {st }}$ Century. American Filtration \& Separation Society, Pittsburgh, Vol. 15, Session No. 29, Hydrocyclone I.

[70] Dueck, J., Schneider, M., Neesse, Th. 2003: Numerical calculation of the umbrella and rope discharge of a hydrocyclone. Aufbereitungstechnik 44, (8), $12-21$.

[71] Trawinski, H. 1977: Anordnung zum fraktionierten Abscheiden von Festsoffen bei einer bestimmten Trennkorngrosse aus Suspesionen mittles Hydrozyklonen. Part DE 2716611C2, Hirschau. 
[72] Neesse, Th., Donhauser, F. 2000: Advances in the theory and practice of hydrocyclone technique. In. Proceedings of the XXI International Mineral Processing Congress, Rome, Italy, A4, 74.

[73] Neesse, Th., Schneider, M., Donhauser, F., Schricker, B. 2001: Computer controlled hydrocyclone battery. In. Chiang, Shiao-Hung, Lee, S. (Eds), Advances in Filtration and Separation Technology, Tampa, FL, American Filtration \& Separation Society, Vol. 15, Session No. 32, Hydrocyclone II.

[74] Schneider, M., Neesse, Th., Schricker, B., Donhauser, F. 2001: Overflow control system for a hydrocyclone battery. In. International Congress of Particle Technology PARTEC, Nuremberg, Session Grinding/Classification/Separation, Nuremberg/Germany; Internal Number: 216.

[75] Peterson, R.D. and Herbst, J.A. 1983: The effects of two-stage hydrocyclone classification on mineral plant performance. Canadian Institution of Mining and Metallurgy, Vol. 23, No.4, 383-391.

[76] Rao, T.C., Bandyopadhyay, A. and Khare, R.N. 1982: Improvement in mill cyclone classifier circuit- a case study. Proc. $14^{\text {th }}$ Int. Min. Proc. Congr., Toronto, Part 3, Paper 7, 1-13.

[77] Firth, B., Hu, S., O’Brien, M., Clarkson, C.J. AND Edward, D. 1998: Some aspects of hydraulic size classification. In. Proc. XIII International Coal Prep. Congress, ed. A. C. Partridge and I. R. Partridge, Australian Coal Preparation Society, 236-244.

[78] Kelsall, D.F., Stewart, P.S.B. and Restarick, C.J. 1974: A practical multiple cyclone arrangements for improved classification. $1^{\text {st }}$ EUR. Conf. Mixing and Centrifugal Separ, Paper E5, 83-93.

[79] Restarick, C.J. 1989: Classification with two-stage cylinder-cyclones in small scale grinding and flotation circuits. Int. J. Min. Proc., 26, 165-179.

[80] Restarick, C.J. 1991: Adjustable on stream classification using two-stage cylinder-cyclones Minerals Engng, 4, 279-288.

[81] Kelsall, D.F. 1963: Some application of hydraulic cyclones in hydrometallurgical processes. Trans. AIME, 226, 225-231. 
[82] Heiskanen, K., Vesanto, A. and Eronen, H. 1987: A high performance hydrocyclone design - the Twin Vortex cyclone. Proc. $3^{\text {rd }}$ Int. Conf. Hydrocyclones, Oxford, BHRA (ed. P. Wood), Elsevier, Amsterdam, Paper K2, 263-268.

[83] Dahlstrom, D.A., 1954: Fundamentals and applications of the liquid cyclone. Chem. Eng. Prog. Symp. Ser., 50, (15), 41-46.

[84] Kelsall, D.F. and Holmes, J.A. 1960: Improvement of classification efficiency in hydraulic cyclones by water injection. PROC. $5^{\text {th }}$ Int. Min. Proc. Congr., Institution of Mining and Metallurgy, London, Paper 9.

[85] Firth, B., Edwards, D., Clarckson, C., and O'Brien, M. 1995: The impact of fine classification on coal preparation performance. In Proc. $7^{\text {th }}$ Australian Coal Preparation Conference, Paper E2, Australian Coal Preparation Society, 250276.

[86] Patil, D.D. and Rao, T.C. 1999: Classification evaluation of water injected hydrocyclone. Minerals Engineering, 12(12), 1527- 1532.

[87] Honaker, R.Q., Ozsever,A.V., Singh, N. and Parekh, B.K. 2001: Apex water injection for improved hydrocyclone classification efficiency. Minerals Engineering, Vol. 14, No. 11, 1445-1457.

[88] Mohanty, M.K., Palit, A. and Dube, B. 2002: A comparative evaluation of new fine particle size separation technologies, Minerals Engineering, 15, 227-236.

[89] Udaya, B.K., Govindarajan, B., Barnwal, J.P., Rao, K.K., Gupta, B.K., Rao, T.C. 2005: Classification studies of lead-zinc ores fines using water-injection cyclone, International Journal of Mineral Processing, 77, 80-94.

[90] Dueck, J., Pikushchak, E., Minkov, L. 2006: On Separation curves of a throughput classification apparatus of finite length. Journal of Engineering Physics and Thermophysics, Vol. 79, No. 4, 171-178.

[91] Dueck, J., Pikushchak, E., Minkov, L. 2007: Modelling of the Fish hook Effect in a classifier. Journal of Engineering Physics and Thermophysics, Vol. 80, No. $1,64-73$.

[92] Dueck, J., Neesse, Th., Minkov, L., Hararah, M. 2004: Theoretical and experimental investigation of distributed settling in a polydisperse suspension. 
Proc. of ICMF-2004. $5^{\text {th }}$ International Conf. on Multiphase Flow, Hosokawa, 30 May- 4 June, Yokohama, Japan, Paper No. 6, 1-8.

[93] Minkov, L., Dueck, J. 2005, Collective effects by settling of polydisperse dense suspension. Eurasian Physical - Technical Journal, Vol. 2, No. 1(3), 47-63.

[94] Kline, S.J., McClintock, F.A. 1953: Describing uncertainties in single sample experiments, Mech. Eng., January 3.

[95] Holman, J.P., Gaida, W.J. 1989: Experimental methods for Engineers. McGrawHill, $5^{\text {th }}$ edition.

[96] Ternovsky, I.G., Kutepov, A.M. 1994: Hydrocycloning. Nauka, Moscow, 350.

[97] Tarjan, G. 1961: Beitrag zur Theorie und Praxis des Hydrocyclone. Aufbereit.Tech., 2(12), 477-483.

[98] Plitt, L.R. 1976: A mathematical model of the hydrocyclone classifier. CIM Bull., Dec., 114-122.

[99] Schubert, H. 1990: Mechanische Verfahrenstechnik, Deutscher Verlag fur Grundstoffindustrie, Leipzig.

[100] Shah, H., Majumder, A.K., Barnwal, J.P. 2005: Minerals Engineering, Vol. 19, 102-104.

[101] Bloor, M.I.G, Ingham, D.B. and Laverack, S.D. 1980: An analysis of boundary layer effects in a hydrocyclone. Proc. $1^{\text {st }}$. Int. Conf. Hydrocyclones, Cambridge, British, Paper 5, 49-62.

[102] Laverack, S.D. 1980: The effect of particle concentration on the boundary layer flow in a hydrocyclone.

[103] Tarr, D.T. 1965: Practical application of liquid cyclones in mineral dressing problems, preprint AIME meeting, Phoenix.

[104] Renner, V.G. AND Cohen, H.E. 1978: Measurments and interpretations of size distribution of particles within a hydrocyclone. Trans. Inst. Min. Metall. Sect. C., 87, C139- C145.

[105] Jull, N.A. 1972: Parameters for cyclone selection. CIM Bull., June, 27-29. 
[106] Mizrahi, J. and Cohen, E. 1966: Studies of some factors influencing the action of hydrocyclones. Trans. INST. Metall. (Sect. C), 75, C318-C330.

[107] Trawinski, H. 1954: Allgemeines über die Andwendungen des Hydrozyklons in der Erzaufbereitung. Z. Erzbergbau Metallhüttenwes., 7 (12), 537-539.

[108] Matschake, D.E., Dahlstrom, D.A. 1959: Miniature hydrocyclones. Part 2. Solid elimination efficiency. Chem. Eng. Prog., 55(1), 79-82. 